\title{
Analysis of Modal Evolution Caused by a Weakly Range-Dependent Seabed in Shallow Water and its Application to Inversion for Geoacoustic Properties
}

by

\section{Kazuhiko Ohta}

B.S. Kyoto University, Japan (1977)

M.S. Massachusetts Institute of Technology (1983)

Submitted in Partial Fulfillment

of the Requirements for the Degree of

Doctor of Philosophy

at the

MASSACHUSETTS INSTITUTE OF TECHNOLOGY

and the

WOODS HOLE OCEANOGRAPHIC INSTITUTION

September 1993

MARINE

BIOLOGICAL

LABORATORY

LIBRARY

WOODS HOLE, MASS.

W. H. O. 1.

(C) Kazuhiko Ohta (1993)

The author hereby grants to MIT and WHOI permission to reproduce and distribute copies of this thesis document in whole or in part.

Signature of Author

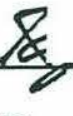

Department of Ocean Engineering, MIT

Department of Applied Ocean Physics and Engineering, WHOI

Certified by

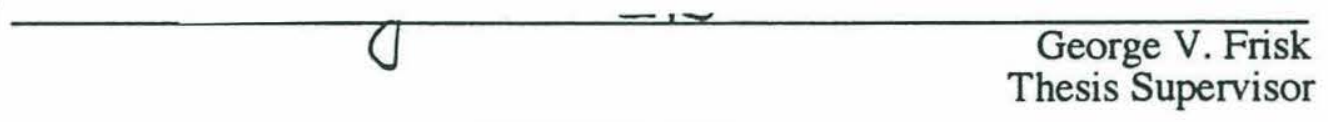

Accepted by

Arthur B. Baggeroer

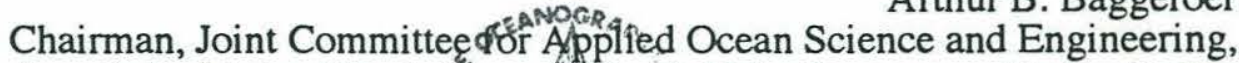
Massachusetts Institute of Techñology/Ayoods Hole Oceanographic Institution

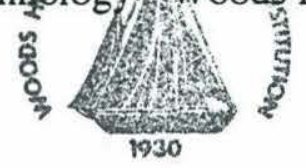




\title{
Analysis of Modal Evolution Caused by a Weakly Range-Dependent Seabed in Shallow Water and its Application to Inversion for Geoacoustic Properties
}

\author{
Kazuhiko Ohta \\ Submitted in partial fulfillment of the requirements \\ for the Degree of Doctor of Philosophy \\ at the \\ MASSACHUSETTS INSTITUTE OF TECHNOLOGY \\ and the \\ WOODS HOLE OCEANOGRAPHIC INSTITUTION
}

September 1993

\begin{abstract}
In a shallow water ocean environment, the range-dependent variation of the geoacoustic properties of the seabed is one of the crucial factors affecting sound propagation. Since the local modes of propagation depend on the spatial changes in the bottom sediments, the local eigenvalues of these modes are useful as tools for examining the range dependence of the sediment properties. In order to extract the local eigenvalues from measurements of the pressure field in a laterally inhomogeneous waveguide, the zero-order asymptotic Hankel transform with a short sliding window is utilized. The local peak positions in the output spectra differ from the local eigenvalues due to both the range variation of the local modes and the interference of adjacent modes. The departure due to the former factor is evaluated analytically by using the stationary phase method. In order to reduce the error induced by the latter factor, mode filtering is utilized by incorporating data from a fixed vertical array of receivers.

The use of the above zero-order Hankel transform in a three-dimensionally varying waveguide results in an underestimate of the local eigenvalues due to the effect of horizontal refraction. Thus a general asymptotic Hankel transform with a 2-D sliding window is used to correct for the underestimated amount. By expanding the latter transform with respect to the azimuthal angle, it can also be shown that the first term in the Taylor series corresponds to the former transform; the rest of the terms account for the value difference between the underestimated and actual local eigenvalues.

In order to obtain the spatial variation of the sediment properties from the rangedependent variation of the extracted local eigenvalues, the analytical relationship between these two variations is derived by using a perturbation method in a horizontally varying, multi-layered bottom model. Upon use of the $n^{2}$-linear profile in each layer, the relationship can be obtained in closed form. As a result, the range variation of the local eigenvalues may be separated into terms that depend on each geoacoustic parameter. Based on this relation, an inversion method for determining the range-dependent geoacoustic parameters is developed.

The methods developed in this thesis are applied to simulated pressure field data as well as experimental field data. It is shown that the evolution with range of the local modes as well as the range-dependent geoacoustic properties can be successfully estimated.

Thesis Supervisor: Dr. George V. Frisk, Senior Scientist, Woods Hole Oceanographic Institution
\end{abstract}




\section{Acknowledgments}

I am deeply grateful to my thesis supervisor, Dr. George Frisk, who suggested the original research topic and guided me throughout this thesis. I could not complete it without his insightful advice, support and encouragement. I would also like to thank the other members of my thesis committee: Prof. Henrik Schmidt, Dr. James Lynch, and Dr. Subramaniam Rajan, who provided me with valuable sources of guidance during the development of this thesis.

I thank Dr. Dajun Tang for his friendship and useful discussion. James Doutt always kindly provided me with the experimental data. Arthur Newhall and Cynthia Sellers helpfully provided expertise in developing the computer programs. My appreciation must be extended to Laura Praderio, who edited most of this thesis. I would also like to express my thanks to Jake Peirson, Abbie Jackson, and the staff in the Education Department.

I am indebted to Prof. Ira Dyer, who encouraged me to pursue the doctoral course and accepted me again as a graduate student at MIT along with financial support in 1990.

I must also acknowledge the Technical Research and Development Institute of the Japanese Defense Agency for providing me the tremendous opportunity of three years of advanced study and financial support during my graduate work.

Finally, I am grateful to my wife, Kyoko, for her help in drafting the figures and for her constant encouragement. I am also indebted to my son, Kengo, and my daughter, Kanako, for their understanding and patience during my graduate work.

Financial support for my research was provided by the WHOI Education Department and the Office of Naval Research. 


\section{Table of Contents}

$\begin{array}{ll}\text { Abstract } & 2\end{array}$

$\begin{array}{ll}\text { Acknowledgments } & 3\end{array}$

Table of Contents

$\begin{array}{ll}\text { 1. Introduction } & 7\end{array}$

$\begin{array}{ll}1.1 \text { Background } & 7\end{array}$

1.2 Thesis Outline $\quad 12$

2. A Review of Modal Representations of the Acoustic Field 14

2.1 Normal mode theory 14

$\begin{array}{ll}2.2 \text { Adiabatic mode theory } & 18\end{array}$

$2.3 \mathrm{Nx} 2 \mathrm{D}$ method $\quad 20$

2.4 Horizontal ray solution $\quad 22$

2.5 Summary 23

3. Detection of Local Eigenvalues in a Laterally Inhomogeneous Waveguide 25

3.1 A review of the Hankel transform 25

3.1.1 The horizontally stratified case 30

3.2 The Hankel transform for a range-dependent medium 33

3.2.1 Peak shift due to range dependence in the local eigenvalue 36

3.2.2 Analysis of simulated data 45

3.3 Application of mode filtering 51

3.4 Analysis of simulated data 61

3.4.1 Ocean and seabed environment 61

3.4.2 Acoustic environment 66

3.4.3 Application of the asymptotic Hankel transform with a sliding window 73

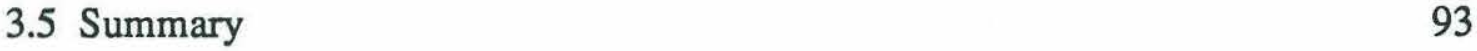

4. Analysis of the Effect of Horizontal Refraction on the Hankel Transform 95

4.1 Problem definition $\quad 95$

4.2 General Hankel transform with a sliding window 98

4.2.1 Derivation of the general Hankel transform with a sliding window $\quad 100$ 
4.2.2 Relationship to the zero-order Hankel transform 103

4.2.3 Analysis of the horizontal refraction effect 105

4.3 Study of the horizontal refraction effect using an alternative representation 112

$\begin{array}{ll}4.4 \text { Analysis of simulated data } & 119\end{array}$

$\begin{array}{ll}\text { 4.4.1 Bottom environment and field simulation } & 119\end{array}$

4.4.2 Application of the general Hankel transform with a sliding window 125

4.5 Experimental Design 140

4.6 Summary 143

5. Inversion Method for the Local Bottom Properties 145

5.1 Geoacoustic model 145

5.2 Relation between perturbed local eigenvalues and perturbed local bottom $\quad 148$ properties

5.3 Range-dependent Pekeris waveguide 155

5.3.1 Derivation of $\Delta \kappa_{n}$ by differentiation of the characteristic equation 159

5.3.2 Derivation of $\Delta \kappa_{n}$ by using the perturbation equation (Eq.(5-24)) 160

5.4 Range-dependent $n^{2}$-linear profile 163

5.4.1 Definition of a range-dependent $n^{2}$-linear profile 164

5.4.2 Evaluation of integrals in the perturbation equation (Eq.(5-24)) 169

5.5 Inversion method to determine range-dependent geoacoustic parameters 173

5.5.1 Formulation of the inverse problem 173

5.5.2 Solution of the inverse problem 175

5.5.3 Reducing the number of unknown parameters 178

5.5.4 Solution of the underdetermined problem 180

5.6 Analysis of simulated data 182

5.6.1 Dependence of the local eigenvalues on the range variation of the 183 geoacoustic parameters

5.6.2 Inversion for the geoacoustic parameters 190

$\begin{array}{ll}5.7 \text { Summary } & 205\end{array}$

6. Analysis of Experimental Data 207

6.1 Nantucket Sound experiment 207

6.2 Hudson Canyon experiment 220

7. Conclusions 239

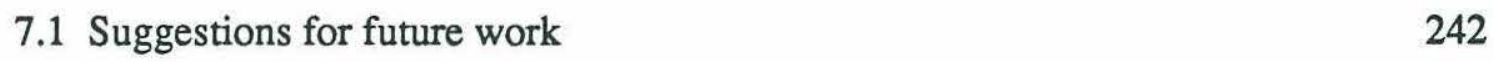


$\begin{array}{ll}\text { Appendix A } & 244\end{array}$

$\begin{array}{lr}\text { Appendix B } & 246\end{array}$

$\begin{array}{lr}\text { Appendix C } & 255\end{array}$

$\begin{array}{lr}\text { Bibliography } & 257\end{array}$ 


\section{Chapter 1}

\section{Introduction}

\subsection{Background}

In ocean acoustics, it is well known that the geoacoustic properties of the seabed play an important role and in shallow water especially become a crucial factor affecting sound propagation in the oceanic waveguide [1-3]. Thus knowledge of these geoacoustic properties is essential not only for acoustic analysis of phenomena in the ocean but also for the design of sonar instrumentation.

A number of studies have been carried out theoretically and experimentally to incorporate the effect of bottom interaction on the acoustic pressure field in the water column (e.g., Refs. 4-16). In the case of the deep ocean, the plane wave reflection coefficient of the bottom is useful for characterizing the acoustic features of the seabed, since bottom-interacting sound can be isolated and interpreted in terms of individual plane wave interactions [17-21]. On the other hand, in shallow water, the reflection coefficient is less appropriate, because the measured field is constructed of many bottominteracting, multipath arrivals, and individual bottom interactions cannot be readily distinguished. In fact, the reflection coefficient and the depth-dependent Green's function are nonlinearly related in shallow water, which leads to an ill-posed problem for determining the reflection coefficient from the measured field $[22,23]$. Thus, instead of using the reflection coefficient, we utilize the normal modes of propagation, which are synthesized from the multipath arrivals, to characterize the effect of the bottom on sound propagation in shallow water.

In general, normal modes can be detected by using vertical or horizontal array $[9,22-$ 33]. In a horizontally stratified waveguide, the modal eigenvalues can be estimated 
accurately from the peaks in the FFT beam-formed output of a horizontal array, which is an approximation to the zero-order Hankel transform relationship between the spatial part of the pressure field and the depth-dependent Green's function [22].

When the sediment properties vary not only vertically but also horizontally, the modal eigenvalues are accordingly subject to change with range. This phenomenon has been confirmed in some measured data from Nantucket Sound (Figure 1-1) [30]. Frisk et al. [30] showed that the splitting of modal spectral peaks suggests the presence two different bottom sound speed profiles (Figure 1-2), which may be associated with different seabed parameters over the surveyed range; the lateral inhomogeneity of this region is assumed to consist of two different range-independent portions in order to apply the modal inverse method. This observation, however, suggests that the information contained in the range-dependent evolution of the local modes can provide a clue to resolving the spatial change of the bottom environment in a continuous manner [32].

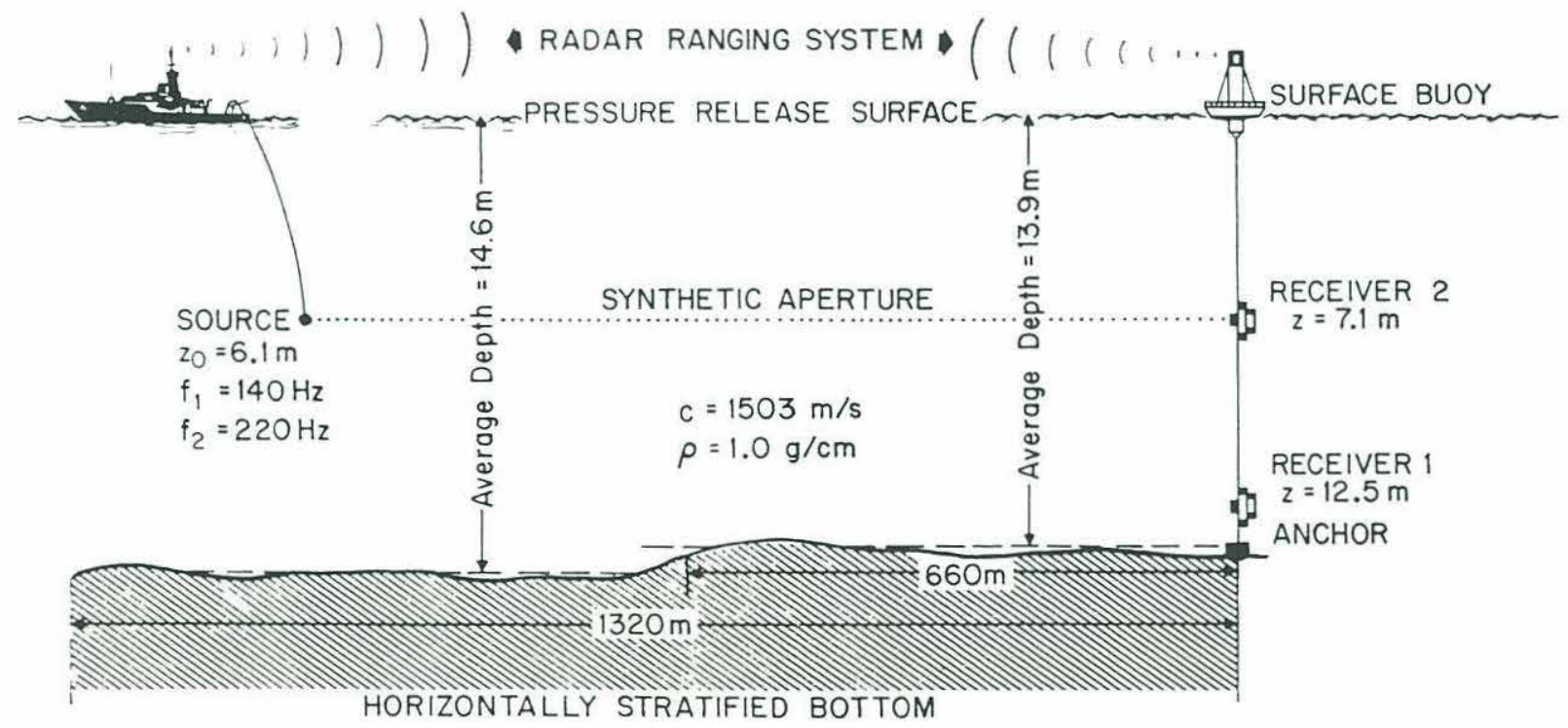

Figure 1-1: Experimental configuration for the Nantucket Sound experiment (from Ref. 30). 


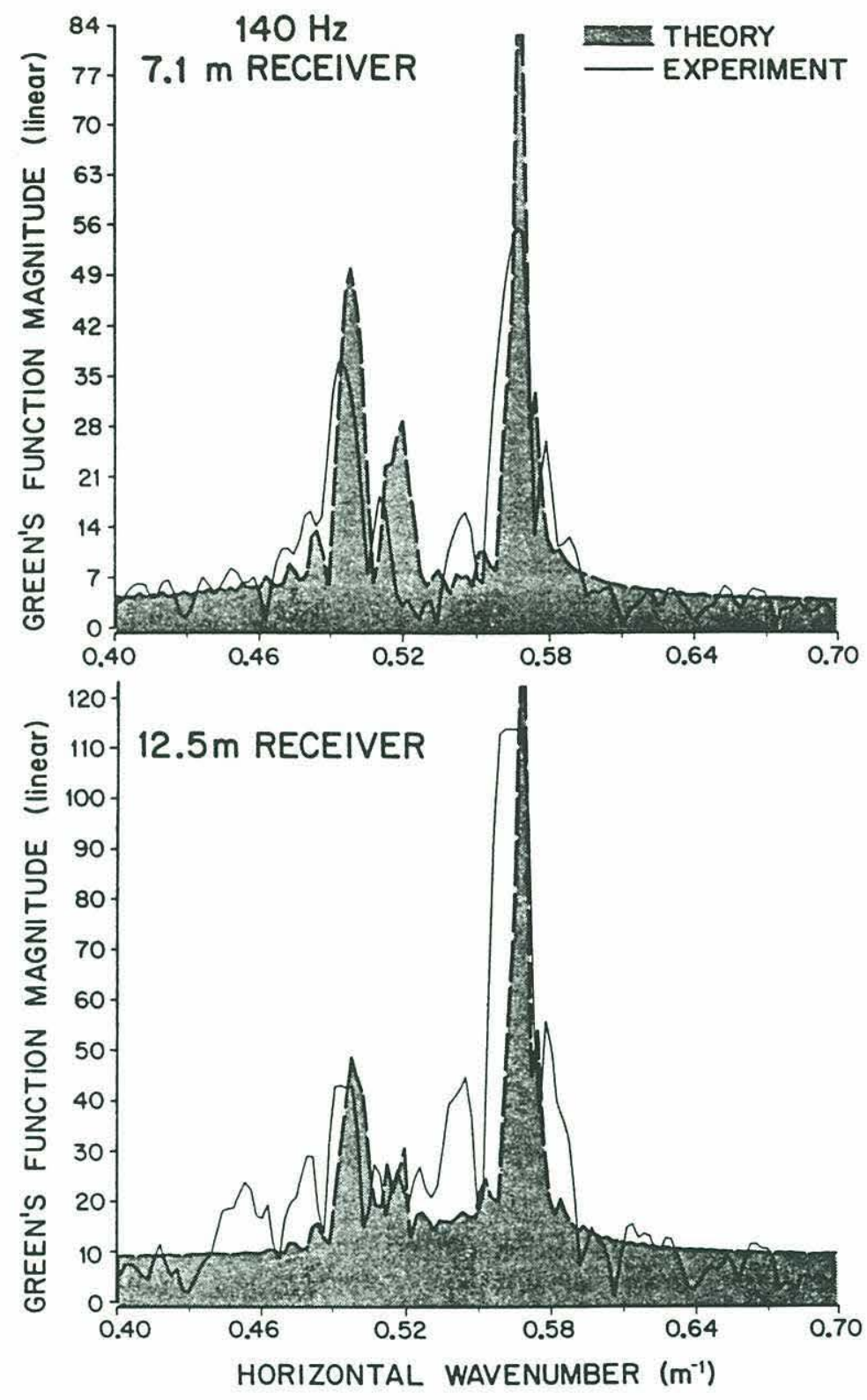

Figure 1-2: Splitting of modal spectral peaks observed in the Nantucket Sound experimental data. This is compared to the Green's function obtained by applying the IFFP to the theoretical pressure fields (from Ref. 30). 
As an approach for estimating the local modes, Prony's method was applied to a laterally varying shallow water waveguide by Diemer [33]. This method is one of several nonlinear spectral estimation methods developed in the last decade. The application of Prony's method was successful to some degree with simulated data, but had only limited success with experimental data.

In this thesis, instead of employing a nonlinear spectral estimation method, a mode separation technique is used as a pre-processing method for the effective use of the Hankel transform with a short sliding window, which is relatively robust to noise. Thus the first half of this thesis is devoted to developing a method for estimating local modes from the pressure field in a shallow water environment having a weakly range-dependent seabed. Then the range-dependent evolution of the local modes will be analyzed to characterize the acoustic features of the waveguide.

Once the local modes are accurately estimated, the next objective is to obtain the spatial variation of the sediment properties from the range-dependent variation of these local modes. Namely, the sediment properties are obtained as a solution of this type of inverse problem. Here it would be worthwhile to review some of the existing inverse methods [34-51].

The iteration of forward models method [34-36] calculates the pressure field by using a numerical model that changes the geoacoustic parameters repeatedly until it best fits the measured pressure field. Although the performance of this method depends on the ability of the numerical model to simulate the field, this method is effective to implement on real experimental data because the results are generally stable with respect to noise. One of the problems with this method has to do with distinguishing local minima from global minimum associated with the best-fit solution. This uniqueness issue becomes significant, when the number of geoacoustic parameters to be determined increases. In order to cope with this higher-dimensional problem, the simulated annealing method [37-40], which is also categorized as an iteration of forward models 
method, is useful and has been applied recently to the measured data at Corpus Christi [40]. This method is a Monte Carlo optimization procedure that numerically imitates the cooling process associated with crystal formation and has become operational practically with the development of high-speed computers. However, these iteration of forward models methods are computationally intensive and time-consuming as compared to other methods.

In contrast, analytic inverse methods do not repeatedly solve a forward problem. Rather, they try to solve a Fredholm integral equation of the first kind, which arises in different forms, depending on the quantities used for the input and output data [41-43]. In exact methods [44], this integral equation may be solved by resorting to the trace method [45] or the Gelfand-Levitan method [46], which was originally developed for inverting the Schrödinger equation [47] for the potential in quantum mechanics. These methods do not require an approximation in the initial stage, so no initial assumptions such as a background model for the geoacoustic parameters are required. But they do involve difficulties in application to real data.

On the other hand, perturbative inversion methods [48-51] linearize the Fredholm integral equation around an initially assumed background model, usually based on the Born approximation [47]. Then the problem becomes tractable, and results established in linear inverse theory can be applied. Thus these perturbative inversion methods have been successfully exploited to determine geoacoustic parameters $[12,29,30]$. Specifically, in the horizontally stratified case, the modal eigenvalues can be utilized in the perturbed integral equation, and they are also robust as input data for the inverse problem $[50,51]$.

In a range-dependent shallow water environment, we first need to clarify the relationship between the range-dependent modal evolution and spatial changes in the geoacoustic properties. To do this, we can expect to utilize the perturbation approach. In 
the latter half of this thesis, an inversion method for obtaining the range-dependent geoacoustic properties will be developed on the basis of this relationship.

\subsection{Thesis Outline}

A primary thrust of my research is to develop a method for extracting the local modes from measurements of the pressure field in a laterally varying waveguide. In addition, concern must be naturally extended to the case of a three-dimensionally (3-D) varying waveguide, where the effect of horizontal refraction has to be taken into consideration. Next, in order to use these extracted local modal eigenvalues in perturbative inversion methods, the relationship between the range-dependent modal evolution and the spatial change in the acoustic properties of the bottom also has to be clarified. Based on this analysis, an inversion method for determining the rangedependent geoacoustic parameters can be established. This thesis deals with these two steps of the inversion problem in an effort to obtain the local geoacoustic properties in a range-dependent shallow water environment.

Chapter 2 reviews the basic features of normal mode theory for a horizontally stratified waveguide and adiabatic mode theory for a range-dependent waveguide. In addition, in order to cope with a 3-D varying waveguide, two methods based on adiabatic modes, i.e., the Nx2D method and the horizontal ray method, are also briefly reviewed. The techniques discussed in this chapter provide the analytical and computational foundation for the remaining of chapters.

In Chapter 3, the asymptotic Hankel transform with a short sliding window is applied to extract the local eigenvalues from measurements of the pressure field in a rangedependent, but cylindrically symmetric, waveguide. A theoretical analysis of the effect of range-varying local eigenvalues in the transform is presented. In an attempt to reduce the error in the transform that is caused by the interference of different modes, mode filtering is employed by incorporating data from a fixed vertical array of receivers. 
Chapter 4 is devoted to detecting the local eigenvalues in a 3-D shallow water environment. We explore the general asymptotic Hankel transform with a sliding window so that we may grasp the effect of horizontal refraction. In particular, this effect is theoretically analyzed in connection with the error that occurs when using the zeroorder asymptotic Hankel transform with a sliding window in non-cylindrical symmetric waveguides.

In Chapter 5, the relationship between the range-dependent variation of the local eigenvalues and the spatial changes in the bottom properties is studied by using a linear perturbation method. Use of an $n^{2}$-linear profile in a multi-layered sediment model enables us to express the above relation in closed form. Based on this relation, the inversion for range-dependent geoacoustic parameters is pursued.

Chapter 6 presents the results obtained by applying the asymptotic Hankel transform with a sliding window to experimental data. Mode filtering is also applied to the pressure field measured by using a fixed vertical array of receivers. Based on the estimated range evolution of the local modes, we discuss the range-dependence of the geoacoustic properties.

In Chapter 7, the conclusions of this research and future work are presented. 


\section{Chapter 2}

\section{A Review of Modal Representations of the Acoustic Field}

As introduced in Chapter 1.1, normal modes are a useful concept to apply to a shallow water environment not only for synthesizing the pressure field in a forward problem but also for analyzing the medium in an inverse problem. In a range-dependent

environment, local modes are uniquely determined by the local properties of the medium, including the bottom sediments. Thus it is natural to expect that one can make use of local modes to infer the range-dependent properties of bottom sediments from measurements of the pressure field.

The purpose of this chapter is to review the normal mode and adiabatic mode theories for describing the acoustic pressure field in shallow water. The analytical representations for the pressure fields based on these theories are useful for development of the modal characterization of the waveguide as well as the theoretical basis for an inverse scheme. The pressure fields simulated by these theories will also be used for computational analysis in the remainder of this thesis. In addition, the Nx2D method and the horizontal ray method are reviewed below. Both methods are used in the simulation of pressure fields for a 3-D varying waveguide in Chapter 4.

\subsection{Normal mode theory}

Normal mode theory in underwater acoustics is one of the principal methods used to provide a full wave solution for sound propagation in a horizontally stratified waveguide and is well suited for shallow water applications [4,13,16,52-54]. 


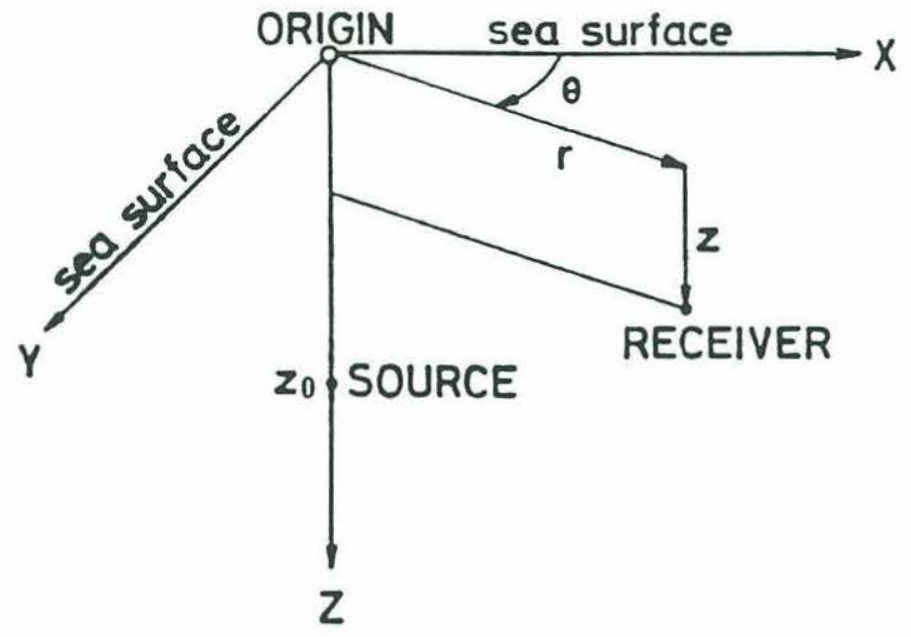

Figure 2-1 : Coordinate system description. 
As shown in Figure 2-1, we set the coordinate system so that $z$ is measured vertically downward from the surface and a point source is located at $x_{0} \equiv\left(0,0, z_{0}\right)$; then the Helmholtz equation for a time-harmonic source with unit strength is expressed as

$$
\rho(x) \nabla \cdot\left[\frac{1}{\rho(x)} \nabla p(x)\right]+k^{2}(x) p(x)=-4 \pi \delta\left(x-x_{0}\right),
$$

where $p(x)$ is the spatial part of the acoustic pressure, $\rho(x)$ is the density, and $k(x)$ is the wavenumber defined by $k(x) \equiv \omega / c(x)$ with sound speed $c(x)$ and circular frequency $\omega$. Here, and throughout this thesis, we assume an $e^{-i \omega t}$ time dependence. Sediment attenuation can be accommodated by adding the attenuation coefficient of sediments, $\alpha(x, \omega)$, to the imaginary part of $k(x)$; i.e., $k(x) \rightarrow k(x)+i \alpha(x, \omega)[53,54]$.

When the medium is horizontally stratified, the Helmholtz equation can be solved by means of conventional normal mode theory. Based on the separation of variables, the solution can be expressed in terms of a sum over a set of eigenfunctions $u_{n}$ of discrete modes plus a branch line integral $I_{B}[55,56]$ :

$$
p\left(r, z, z_{0}\right)=\frac{i \pi}{\rho\left(z_{0}\right)} \sum_{n} u_{n}\left(z_{0}\right) u_{n}(z) H_{0}^{(1)}\left(\kappa_{n} r\right)+I_{B},
$$

in which $H_{0}^{(1)}$ represents the zero order Hankel function of the first kind. Here, owing to the symmetry of the pressure field around the source, a cylindrical coordinate system such that $r \equiv \sqrt{x^{2}+y^{2}}$ has been used.

The $n$th mode eigenfunction, $u_{n}(z)$, satisfies the depth equation

$$
\rho(z) \frac{d}{d z}\left(\frac{1}{\rho(z)} \frac{d u_{n}}{d z}\right)+\left[k^{2}(z)-\kappa_{n}^{2}\right] u_{n}=0
$$

together with the prescribed boundary conditions. The eigenfunctions are normalized so as to satisfy the orthonormality condition: 


$$
\int_{0}^{\infty} \frac{1}{\rho(z)} u_{n}(z) u_{m}(z) d z=\delta_{m m},
$$

where $\delta_{n m}$ is the Kronecker-delta and the inverse of the density serves as a weighting function. In Eq.(2-3), $\kappa_{n}$ stands for the eigenvalue of the $n$th mode, which is determined uniquely by solving a characteristic equation subject to the boundary conditions. Physically, the real part of $\kappa_{n}$ is identified as the horizontal component of the wavenumber and its imaginary part characterizes the attenuation rate of mode energy in the propagation process.

The branch line integral, $I_{B}$, arises when the bottom structure is terminated with a fast isovelocity half-space. Since $I_{B}$ usually decreases rapidly with range, its contribution to the far-field pressure in Eq.(2-2) is often neglected [55,56].

In this range of interest, $H_{0}^{(1)}\left(\kappa_{n} r\right)$ can generally be approximated by its asymptotic form [57]:

$$
H_{0}^{(1)}\left(\kappa_{n} r\right) \sim \sqrt{\frac{2}{\pi \kappa_{n} r}} e^{i\left(\kappa_{n} r-\pi / 4\right)},
$$

so that Eq.(2-2) can be expressed as

$$
p(r, z) \sim e^{-i \frac{\pi}{4}} \sqrt{\frac{2 \pi}{r}} \sum_{n} \frac{1}{\sqrt{\kappa_{n}}} u_{n}\left(z_{0}\right) u_{n}(z) e^{i \kappa_{n} r} \quad\left(\kappa_{n} r>>1\right),
$$

where the density in the water has been set to $1 \mathrm{~g} / \mathrm{cm}^{3}, \rho\left(z_{0}\right)=1$. This assumption will not incur a large error in shallow water.

As mentioned before, the normal mode representation is applicable only to the horizontally stratified waveguide. Thus let us consider an approximate mode theory applicable to range-dependent waveguides in the next section. 


\subsection{Adiabatic mode theory}

When the ocean environment, including the bottom sediment, varies gradually in the horizontal direction, adiabatic mode theory is effective in representing the acoustic field. The first to point out the adiabatic propagation process in underwater acoustics was Weston [58], and then Milder [59] demonstrated elegantly, from an analogy with classical mechanics, that the adiabatic invariant corresponds to the mode number. In waveguides for which the range-dependent variation is gradual enough to apply adiabatic mode theory, the acoustic energy is transported in the horizontal direction separately by noninteracting modes [60]. In other words, the coupling between different modes that is induced by the range variation of the medium has to be small enough to be neglected in adiabatic mode theory [59].

In this method, we first assume that the solution of the Helmholtz equation in Eq.(21) can be expanded in terms of local eigenfunctions $u_{n}(x, y, z)$ as

$$
p(x, y, z)=\sum_{n} R_{n}(x, y) u_{n}(x, y, z),
$$

where $R_{n}(x, y)$ is an unknown range function to be determined and $u_{n}(x, y, z)$ are defined so as to satisfy the following depth equation $[16,61,62]$ :

$$
\rho(x, y, z) \frac{\partial}{\partial z}\left(\frac{1}{\rho(x, y, z)} \frac{\partial u_{n}}{\partial z}\right)+\left[k^{2}(x, y, z)-\kappa_{n}^{2}(x, y)\right] u_{n}=0
$$

with given boundary conditions at each range. Here, $\kappa_{n}(x, y)$ is called a local eigenvalue, which is a function of horizontal position. In a manner similar to conventional mode theory, the $u_{n}(x, y, z)$ satisfy an orthonormality condition:

$$
\int_{0}^{\infty} \frac{1}{\rho(x, y, z)} u_{n}(x, y, z) u_{m}(x, y, z) d z=\delta_{n m} .
$$


The orthonormality property of the local eigenfunctions provides the foundation of mode filtering as will be discussed in Chapter 3.3.

Substituting Eq.(2-7) into the Helmholtz equation and utilizing the orthonormality condition in Eq.(2-9) yield a coupled equation for the range function $R_{n}[5,62]$. Coupling terms appearing in this equation are induced by the horizontal variation of the medium and reflect the fact that the redistribution of modal energy evolves in the propagation process [60]. Due to the current assumption of weak range dependence, these coupling terms may be dropped out of the coupled equation, yielding a range equation for $R_{n}$ for each mode:

$$
\nabla_{\perp}^{2} R_{n}(x, y)+\kappa_{n}^{2}(x, y) R_{n}(x, y)=-\frac{2}{r} u_{n}\left(0,0, z_{0}\right) \delta(r),
$$

where $\vec{\nabla}_{\perp} \equiv\left(\frac{\partial}{\partial x}, \frac{\partial}{\partial x}\right)$. This procedure is commonly called the adiabatic approximation $[59,61]$.

If the waveguide medium is cylindrically symmetric around the source, i.e., the horizontal variation of the medium only depends on the range from the source, then it is found immediately from Eq.(2-10) that the pressure field also depends on the range only. In this case, a cylindrical coordinate system is more manageable to describe the range equation, giving

$$
\frac{1}{r} \frac{d}{d r}\left(r \frac{d R_{n}(r)}{d r}\right)+\kappa_{n}^{2}(r) R_{n}(r)=-\frac{2}{r} u_{n}\left(0, z_{0}\right) \delta(r) \quad\left(\kappa_{n} r>>1\right) .
$$

At this point, by exploiting the WKBJ approximation method and matching the boundary condition close to the source, we obtain an adiabatic mode solution in the following form $[62,63]$ : 


$$
p\left(r, z, z_{0}\right)=e^{-i \frac{\pi}{4}} \sqrt{\frac{2 \pi}{r}} \sum_{n} \frac{1}{\sqrt{\kappa_{n}(r)}} u_{n}\left(0, z_{0}\right) u_{n}(r, z) e^{i \int_{0}^{r} \kappa_{n}(r) d r} \quad\left(\kappa_{n} r \gg>1\right) .
$$

As seen from a comparison of the field given by normal mode sum in Eq.(2-6) and the field given by adiabatic mode sum in Eq.(2-12), the major distinction between them arises principally from the difference in the phase factor associated with each mode. This is also a key factor when detecting the eigenvalue from the pressure field in the inverse process, as will be discussed in Chapter 3.2.

When the waveguide is not cylindrically symmetric, we have to start from Eq.(2-10) to obtain the pressure field. The next two sections deal with this problem.

\section{$2.3 \mathrm{~N} \times 2 \mathrm{D}$ method}

In order to develop a method for extracting local eigenvalues in a 3-D varying environment in Chapter 4, we need to simulate the pressure field in this environment; the $\mathrm{N} \times 2 \mathrm{D}$ method $[64,65]$ is one of the methods for realizing it.

If the cylindrical symmetry of the waveguide breaks down, then Eq.(2-12) is no longer a solution of Eq.(2-10). To describe the field correctly in this 3-D, yet adiabatically varying medium, we have to solve Eq.(2-10) or, equivalently, the equation incorporating the azimuth angle into Eq.(2-11) such that

$$
\frac{1}{r} \frac{\partial}{\partial r}\left(r \frac{\partial R_{n}(r, \theta)}{\partial r}\right)+\frac{1}{r^{2}} \frac{\partial^{2} R_{n}(r, \theta)}{\partial \theta^{2}}+\kappa_{n}^{2}(r, \theta) R_{n}(r, \theta)=-\frac{2}{r} u_{n}\left(0,0, z_{0}\right) \delta(r) .
$$

But this is not so straightforward. Instead of doing this, the Nx2D method tries to solve Eq.(2-13) by treating the medium as if each medium sliced in the $\theta$ direction were cylindrically symmetric. To be explicit, the Nx2D method approximates Eq.(2-13) by the following equation: 


$$
\frac{1}{r} \frac{\partial}{\partial r}\left(r \frac{\partial R_{n}(r, \theta)}{\partial r}\right)+\kappa_{n}^{2}(r, \theta) R_{n}(r, \theta)=-\frac{2}{r} u_{n}\left(0,0, z_{0}\right) \delta(r),
$$

and, consequently, provides an approximate solution for $p(r, \theta, z)$ :

$$
p\left(r, \theta, z, z_{0}\right)=e^{-i \frac{\pi}{4}} \sqrt{\frac{2 \pi}{r}} \sum_{n} \frac{1}{\sqrt{\kappa_{n}(r, \theta)}} u_{n}\left(0,0, z_{0}\right) u_{n}(r, \theta, z) e^{i \int_{0}^{r} \kappa_{n}(r, \theta) d r} .
$$

Namely, the Nx2D method takes into account the azimuthal dependence of the field when deriving the local eigenfunctions in the first stage, but neglects the cross-angle variation for $R_{n}$ when solving the range equation in the next stage. It should be noted, however, that $R_{n}$ still keeps the azimuthal dependence through $\kappa_{n}(r, \theta)$ as an outcome. Thus, the azimuth angle $\theta$ simply plays a role as a parameter in Eq.(2-14). This can also be recognized by a comparison of Eq.(2-12) and Eq.(2-15).

In spite of no direct interaction between the different azimuth directions, as indicated in Eq.(2-14), the azimuthal variation of $p(r, \theta, z)$ in Eq.(2-15) produces the same effect as if the phase front were redirected in a different direction from $\theta$. Hence, we can see that the effect of horizontal refraction is included to some extent in Eq.(2-15), although it is not completely correct.

The Nx2D approach was also employed successfully by Perkins et al. [64] to apply the parabolic equation method to a 3-D varying waveguide, which has stronger variation than that dealt with here. He demonstrated the effectiveness of this approach in both an analytical and numerical manner.

When simulating the pressure field in a 3-D varying environment in Chapter 4, the NX2D method will be compared to the horizontal ray method, which is briefly reviewed in the next section. 


\subsection{Horizontal Ray method}

As an alternative approach for representing the pressure field in a 3-D varying waveguide, one can employ the horizontal ray method. In this approach, adiabatic mode theory is first applied to the vertical direction in order to obtain local eigenfunctions at each range. Then, in the next stage, the ray method is utilized in the horizontal plane to grasp the evolution of local modes. This idea was originally employed by Pierce [61], then was further developed in a more general manner by Weinberg et al. [66], by exploiting asymptotic series. To implement the horizontal ray method, the horizontal variation of the medium has to be small, especially compared to the vertical variation.

The advantage of this approach is that it affords a clear picture of the horizontal refraction for each mode. In shallow water, this refraction is caused not only by bathymetric change but also by the variation in sediment properties and layer structure; the degree of this refraction also depends on the mode and frequency of interest. Hence, the effect of horizontal refraction cannot be neglected in the inverse problem for the 3-D varying bottom environment.

If we confine ourselves to the zero order term of the asymptotic series in horizontal ray theory, the phase $\Phi_{n}$ in $R_{n}\left(\equiv A_{n} e^{i \Phi_{n}}\right)$ has to satisfy an eikonal equation, which subsequently leads to a ray equation:

$$
\begin{aligned}
& \frac{d}{d s}\left(\kappa_{n} \cos \theta_{n}\right)=\frac{\partial \kappa_{n}}{\partial x}, \\
& \frac{d}{d s}\left(\kappa_{n} \sin \theta_{n}\right)=\frac{\partial \kappa_{n}}{\partial y},
\end{aligned}
$$

where $d s$ is an increment of length along the horizontal ray path of the $n$th mode and $\theta_{n}$ indicates the direction perpendicular to the phase front ( $\Phi_{n}=$ constant) at the position $(x, y)$. A set of these equations clearly indicates that the horizontal refraction of the mode is characterized by the spatial variation of its local eigenvalue. Based on both the ray 
equation in Eq.(2-16) and the transport equation for the amplitude $A_{n}$, the pressure field can be represented to the leading order as $[61,67]$

$$
p\left(x, y, z, z_{0}\right)=e^{-i \frac{\pi}{4}} \sqrt{2 \pi} \sum_{n} \frac{1}{\sqrt{D_{n}}} u_{n}\left(0,0, z_{0}\right) u_{n}(x, y, z) e^{i \int_{0}^{(z x)} k_{n} d s}
$$

where the integral in the phase term is taken along the horizontal ray path determined

from Eq.(2-16) and $D_{n} \equiv \frac{\partial(x, y)}{\partial(s, \theta)}$ is the Jacobian for transformation from Cartesian coordinates to ray coordinates $(s, \theta)$. In the cylindrically symmetric case, $D_{n}=\kappa_{n} r$ and $d s=d r$, so that Eq.(2-17) reduces to Eq.(2-12).

The effect of horizontal refraction on the inversion process for detecting the local eigenvalues will be discussed in Chapter 4. Also, the pressure field in Eq.(2-17) will be utilized to synthesize the acoustic field for the numerical study.

\subsection{Summary}

In this chapter, the basic features of the conventional and adiabatic mode theory were reviewed as were the Nx2D method and the horizontal ray method.

Conventional mode theory is of considerable utility in shallow water but can be applied exactly only to the horizontally stratified case. On the other hand, adiabatic mode theory can be applied to a range-dependent waveguide as long as the waveguide medium changes gradually in the horizontal direction. In the case of a cylindrically symmetric waveguide, the most prominent difference in the representation of the pressure field in these two theories is the phase factor associated with each mode; the former is provided simply by the eigenvalue multiplied by the range, whereas the latter is given by the integration of the local eigenvalue with respect to range.

Both the Nx2D method and the horizontal ray method are designed to cope with a 
3-D varying waveguide. In the Nx2D method, the waveguide is treated as if each medium sliced in the azimuthal direction were cylindrically symmetric; then, for each direction one can make use of the results of the adiabatic mode sum that is derived for the radially inhomogeneous waveguide. On the other hand, the horizontal ray method can deal with the phase factor more exactly by tracing the modal evolution on a horizontal plane after obtaining local modes at each range.

The methods reviewed in this chapter will provide the analytical and computational foundation in the remainder of this thesis. 


\section{Chapter 3 \\ Detection of Local Eigenvalues in a Laterally \\ Inhomogeneous Waveguide}

The purpose of this chapter is to extract the local eigenvalues from a given pressure field in a laterally inhomogeneous waveguide. Before doing this, we will first review the Hankel transform, whose asymptotic form can provide exact eigenvalues of normal modes in a horizontally stratified waveguide $[16,22]$. Then, a sliding window is introduced into an asymptotic Hankel transform of order zero in order to cope with a laterally inhomogeneous waveguide. The effect of range variation in the local eigenvalues on this transform is examined in an analytical manner in Section 3.2. In an attempt to improve the processing in this transform in a multi-mode environment, the application of mode filtering is explored in Section 3.3 by incorporating data from a fixed vertical array of receivers. These results are studied in Section 3.4 by using pressure fields that are simulated with the use of adiabatic mode theory. Note that the waveguide is assumed to be cylindrically symmetric about the $z$ axis throughout this chapter. A noncylindrically symmetric case will be dealt with in Chapter 4 .

\subsection{A review of the Hankel Transform}

In a horizontally stratified waveguide, eigenvalues of normal modes can be accurately detected by an asymptotic Hankel transform of order zero. In this section, the Hankel transform is first reviewed and then its relationship to the depth-dependent Green's function is presented in connection with the eigenvalues of conventional mode theory. 
To begin with, we will take a two-dimensional (2-D) Fourier transform of the spatial part of the acoustic pressure $p(x, y, z)$ such that

$$
\tilde{g}\left(k_{x}, k_{y} ; z\right)=\int_{-\infty}^{\infty} \int_{-\infty}^{\infty} p(x, y, z) e^{-i\left(k_{x} x+k_{y} y\right)} d x d y,
$$

where $\left(k_{x}, k_{y}\right)$ are the horizontal components of the wavenumber and $\tilde{g}\left(k_{x}, k_{y} ; z\right)$ is generally referred to as a wavenumber spectrum. Note that this spectrum still retains its dependence on the depth variable $z$ and, therefore, must be distinguished from the wavenumber spectrum that is defined by a three-dimensional (3-D) Fourier transform, which is completely independent of spatial coordinates. The inverse Fourier transform associated with Eq.(3-1) is given by

$$
p(x, y, z)=\frac{1}{(2 \pi)^{2}} \int_{-\infty}^{\infty} \int_{-\infty}^{\infty} \tilde{g}\left(k_{x}, k_{y} ; z\right) e^{i\left(k_{x} x+k_{y} y\right)} d k_{x} d k_{y} .
$$

Here we may rewrite the above transform pairs in terms of cylindrical coordinates in both the space and the wavenumber domains through

$$
\begin{array}{lll}
x=r \cos \theta & y=r \sin \theta & r=\sqrt{x^{2}+y^{2}} \\
k_{x}=k_{r} \cos \varphi & k_{y}=k_{r} \sin \varphi & k_{r}=\sqrt{k_{x}^{2}+k_{y}^{2}} .
\end{array}
$$

It follows that

$$
\tilde{g}\left(k_{r}, \varphi ; z\right)=\int_{0}^{\infty} d r r \int_{0}^{2 \pi} d \theta p(r, \theta, z) e^{-i k, r \cos (\theta-\varphi)},
$$

and

$$
p(r, \theta, z)=\frac{1}{(2 \pi)^{2}} \int_{0}^{\infty} d k_{r} k_{r} \int_{0}^{2 \pi} d \varphi \tilde{g}\left(k_{r}, \varphi ; z\right) e^{i k r \cos (\theta-\varphi)} .
$$


A general Hankel transform can be deduced from this form, but in this chapter let us confine ourselves to cylindrically symmetric fields only. In Chapter 4 , we will discuss the application of the general Hankel transform to non-cylindrically symmetric fields in connection with horizontal refraction effects.

When the pressure field $p$ is cylindrically symmetric, we can take $p$ out of the integral with respect to $\theta$, giving

$$
\tilde{g}\left(k_{r} ; z\right)=\int_{0}^{\infty} d r r p(r, z) \int_{0}^{2 \pi} d \theta e^{-i k, r \cos (\theta-\varphi)} .
$$

Use of the following integral representation for the zero-order Bessel function [57]

$$
J_{0}(\zeta)=\frac{1}{2 \pi} \int_{0}^{2 \pi} e^{i \zeta \cos (\alpha-\beta)} d \alpha
$$

in Eq.(3-6) yields

$$
\tilde{g}\left(k_{r} ; z\right)=2 \pi \int_{0} p(r, z) J_{0}\left(k_{r} r\right) r d r,
$$

or, alternatively,

$$
g\left(k_{r} ; z\right)=\int_{0}^{\infty} p(r, z) J_{0}\left(k_{r} r\right) r d r,
$$

in which we have introduced the new wavenumber spectrum $g\left(k_{r} ; z\right)$ that is defined by

$$
g\left(k_{r} ; z\right)=\frac{1}{2 \pi} \tilde{g}\left(k_{r} ; z\right) .
$$

Eq.(3-9) is commonly called a zero-order Hankel transform [16].

Likewise, due to the cylindrical symmetry of $g$ in the wavenumber domain, Eq.(3-5) is reduced to an inverse zero-order Hankel transform: 


$$
p(r, z)=\int_{0} g\left(k_{r} ; z\right) J_{0}\left(k_{r} r\right) k_{r} d k_{r}
$$

The conjugate transform pairs in Eq.(3-10) and Eq.(3-11) exhibit that the Hankel transform and its inverse have the same form. It is also observed that both $p(r, z)$ and $g\left(k_{r} ; z\right)$ become even functions in terms of $r$ and $k_{r}$, respectively, since $J_{0}\left(k_{r} r\right)$ is an even function.

The transform pairs described above can also be expressed using the Hankel function. To do this, the following identity [57] is substituted into Eq.(3-11):

$$
J_{0}\left(k_{r} r\right)=\frac{1}{2}\left[H_{0}^{(1)}\left(k_{r} r\right)+H_{0}^{(2)}\left(k_{r} r\right)\right]
$$

yielding

$$
p(r, z)=\frac{1}{2} \int_{0} g\left(k_{r} ; z\right) H_{0}^{(1)}\left(k_{r} r\right) k_{r} d k_{r}+\frac{1}{2} \int_{0} g\left(k_{r} ; z\right) H_{0}^{(2)}\left(k_{r} r\right) k_{r} d k_{r}
$$

Letting $k_{r}=-\xi$ in the second integral leads to

$$
p(r, z)=\frac{1}{2} \int_{0}^{\infty} g\left(k_{r} ; z\right) H_{0}^{(1)}\left(k_{r} r\right) k_{r} d k_{r}+\frac{1}{2} \int_{0}^{\infty} g(-\xi ; z) H_{0}^{(2)}(-\xi r) \xi d \xi
$$

Using $g(\xi ; z)=g(-\xi ; z)$ and $H_{0}^{(2)}(-\xi r)=-H_{0}^{(1)}(\xi r)$ [57] in the second integral in Eq.(314) results in

$$
p(r, z)=\frac{1}{2} \int_{-\infty}^{\infty} g\left(k_{r} ; z\right) H_{0}^{(1)}\left(k_{r} r\right) k_{r} d k_{r} \quad(r>0) .
$$

As detailed in Ref. 23, this representation is valid only for $r>0$. In the same manner, we can obtain the conjugate transform: 


$$
g\left(k_{r} ; z\right)=\frac{1}{2} \int_{-\infty}^{\infty} p(r, z) H_{0}^{(2)}\left(k_{r} r\right) r d r \quad\left(k_{r}>0\right) .
$$

If the observation range corresponds to $k_{r} r \gg 1$, then we can employ the asymptotic form of the Hankel function [57] in Eq.(3-15) and Eq.(3-16):

$$
\begin{aligned}
& H_{0}^{(1)}\left(k_{r} r\right) \sim \sqrt{\frac{2}{\pi k_{r} r}} e^{i(k, r-\pi / 4)}, \\
& H_{0}^{(2)}\left(k_{r} r\right) \sim \sqrt{\frac{2}{\pi k_{r} r}} e^{-i\left(k_{r} r-\pi / 4\right)} .
\end{aligned}
$$

Therefore a pair of asymptotic Hankel transforms can be expressed as

$$
\begin{aligned}
& p(r, z) \sim \frac{e^{-i \pi / 4}}{\sqrt{2 \pi r}} \int_{-\infty} g\left(k_{r} ; z\right) \sqrt{k_{r}} e^{i k r} d k_{r}, \\
& g\left(k_{r} ; z\right) \sim \frac{e^{i \pi / 4}}{\sqrt{2 \pi k_{r}}} \int_{-\infty} p(r, z) \sqrt{r} e^{-i k, r} d r .
\end{aligned}
$$

These integral forms enable us to utilize the Fast Fourier Transform (FFT), which is useful when numerically evaluating the integrals in Eq.(3-19) and Eq.(3-20). Equation (3.19) is the basis of Fast Field Programs (FFP) for computing the sound field $[68,69]$ In order to use Eq.(3-20), the only assumption we have made about $p(r, z)$ is that it be cylindrically symmetric. Hence, even in a laterally inhomogeneous waveguide, $g\left(k_{r} ; z\right)$ can be clearly defined by applying the above asymptotic Hankel transform (Eq.(3-20)) to the pressure field in this waveguide, as long as the field is cylindrically symmetric.

Here it is of particular interest to examine the wavenumber spectrum $g\left(k_{r} ; z\right)$ in Eq.(3-20) for the case of a horizontally stratified waveguide. 


\subsubsection{The horizontally stratified case}

At this point, let us demonstrate that $g\left(k_{r} ; z\right)$ given by Eq.(3-20) corresponds to a depth-dependent Green's function if, and only if, $p(r, z)$ is the pressure field obtained in a horizontally stratified medium [16]. In that case, $p(r, z)$ satisfies the inhomogeneous Helmholtz equation described in terms of cylindrical coordinates as

$$
\begin{aligned}
\frac{1}{r} \frac{\partial}{\partial r}\left(r \frac{\partial}{\partial r} p(r, z)\right)+\rho(z) \frac{\partial}{\partial z}\left[\frac{1}{\rho(z)} \frac{\partial}{\partial z} p(r, z)\right]+ & k^{2}(z) p(r, z) \\
& =-4 \pi \frac{\delta(r)}{r} \delta\left(z-z_{0}\right) .
\end{aligned}
$$

By applying the zero-order Hankel transform operator $\int_{0} d r J_{0}\left(k_{r} r\right) r$ to both sides of Eq.(3-21) and employing the following relation in the first term

$$
\int_{0}^{\infty} d r J_{0}\left(k_{r} r\right) r\left[\frac{1}{r} \frac{\partial}{\partial r}\left(r \frac{\partial}{\partial r} p(r, z)\right)\right]=-k_{r}^{2} \tilde{g}\left(k_{r} ; z\right)
$$

we obtain

$$
\rho(z) \frac{d}{d z}\left[\frac{1}{\rho(z)} \frac{d}{d z} g\left(k_{r} ; z\right)\right]+\left[k^{2}(z)-k_{r}^{2}\right] g\left(k_{r} ; z\right)=-2 \delta\left(z-z_{0}\right),
$$

where the spectrum $g$ defined in Eq.(3-10) has again been used instead of $\tilde{g}$.

For a fixed value of the wavenumber $k_{r}$, the spectrum $g\left(k_{r} ; z\right)$ is the solution which satisfies the differential equation for a point source (Eq.(3-23)), subject to properly posed boundary conditions. Therefore, $g\left(k_{r} ; z\right)$ is called a depth-dependent Green's function and is conventionally expressed by $g\left(k_{r} ; z, z_{0}\right)$ to emphasize its dependence on the source depth $z_{0}$ as well. 
Once $g\left(k_{r} ; z, z_{0}\right)$ is obtained by solving Eq.(3-23), we can construct the pressure field by substituting $g\left(k_{r} ; z, z_{0}\right)$ into Eq.(3-11). This integral representation can also be transformed into the modal representation in Eq.(2-2) by using Cauchy's residue theorem. To do this, we allow $k_{r}$ to become complex and deform the integration path in the complex- $k_{r}$ plane so as to enclose the poles of the Green's function $g\left(k_{r} ; z, z_{0}\right)$, which correspond to the eigenvalues of the discrete modes. Owing to Cauchy's residue theorem [70], the integral in Eq.(3-15) becomes a sum of residue contributions, each of which is expressed as a modal eigenfunction. Furthermore, if a branch cut exists, then a contribution from the integral around the branch cut must be added. As a result, the pressure field can be expressed as a sum of discrete modes plus a branch line integral as given by Eq.(2-2) in Chapter $2 \cdot 1[16,52,55,56]$.

Conversely, let us apply the asymptotic Hankel transform to the pressure field $p(r, z)$ that was synthesized in Eq.(2-6) for $k_{r} r \gg>1$, as explained in Chapter 2.1. When $p(r, z)$ is obtained over a finite range $(0<r<R)$, as is the case in actual experiments, the asymptotic Hankel transform in Eq.(3-20) has to be computed over a finite aperture $R$ :

$$
g\left(k_{r} ; z\right) \sim \frac{e^{i \pi / 4}}{\sqrt{2 \pi k_{r}}} \int_{0}^{R} p(r, z) \sqrt{r} e^{-i k, r} d r
$$

Equivalently, by using a window function $w_{R}(r)$ that is defined as

$$
w_{R}(r)=\left\{\begin{array}{lc}
1 & (0 \leq r \leq R) \\
0 & (r<0, R<r)
\end{array},\right.
$$

Eq.(3-24) can be rewritten as

$$
g\left(k_{r} ; z\right) \sim \frac{e^{i \pi / 4}}{\sqrt{2 \pi k_{r}}} \int_{-\infty}^{\infty} w_{R}(r) p(r, z) \sqrt{r} e^{-i k r r} d r
$$


Substituting the pressure field given by Eq.(2-6) into Eq.(3-24) and changing the order of the Fourier integration and the mode summation leads to

$$
g\left(k_{r} ; z, z_{0}\right) \sim \frac{i}{\sqrt{k_{r}}} \sum_{n} u_{n}\left(z_{0}\right) u_{n}(z) \int_{0}^{R} e^{-i\left(k-k_{n}\right) r} d r
$$

By executing the integral, Eq.(3-27) results in

$$
g\left(k_{r} ; z, z_{0}\right) \sim \frac{i}{\sqrt{k_{r}}} \sum_{n} u_{n}\left(z_{0}\right) u_{n}(z) \frac{e^{-i\left(k_{r}-K_{n}\right) R}-1}{k_{r}-\kappa_{n}} .
$$

This result shows that the Green's function has peaks at $k_{r}=k_{n}\left(\equiv \operatorname{Re}\left[\kappa_{n}\right]\right)$, i.e., the real part of the mode eigenvalues, which are, however, finite in width and amplitude. This is due to the effects of the finite aperture $R$ and the modal attenuation $\beta_{n} \equiv \operatorname{Im}\left[\kappa_{n}\right]$. The amplitudes are also proportional to the values of the eigenfunctions at the source and receiver depths. Thus one can accurately determine the eigenvalues of the normal modes from the FFT beamformed output of a finite-aperture horizontal array, as long as the source and receiver depths are not close to the null of modes and the eigenvalues of adjacent of modes are not too close. The equivalent result can also be obtained by applying Eq.(3-26) to the same pressure field Eq.(2-6), yielding

$$
g\left(k_{r} ; z, z_{0}\right) \sim \frac{i}{\sqrt{k_{r}}} \sum_{n} u_{n}\left(z_{0}\right) u_{n}(z) \mathfrak{I}\left\{w_{L}(r)\right\} * \mathfrak{I}\left\{e^{-\beta_{n}|r| r}\right\} * \mathfrak{S}\left\{e^{i k_{n} r}\right\}
$$

where $\mathfrak{I}\{\bullet\} \equiv \int_{-\infty}^{\infty} d r e^{i k_{r} r}$ represents a Fourier transform operator and $*$ indicates a convolution operation.

Thus, in a horizontally stratified environment, we can obtain accurate estimates of modal eigenvalues by identifying peaks in the wavenumber spectrum which is obtained by taking the zero-order asymptotic Hankel transform of the pressure field obtained over a finite aperture. 


\subsection{The Hankel transform for a range-dependent medium}

In the horizontally stratified case, as demonstrated in the previous section, the modal eigenvalues can be obtained accurately from the peaks in the FFT beamformed output of a finite-aperture horizontal array. In Ref. 30, this approach was extended to a weakly range-dependent case by discretizing a laterally inhomogeneous waveguide into a finite number of segments whose properties are range-independent. This scheme may be generalized further by introducing the technique of the Short-Time Fourier Transform (STFT) with a short sliding window $w_{L}(r ; \hat{r})$, in which $\hat{r}$ and $L$ indicate the center position and window length of the sliding window, respectively $[32,71,72]$. The spectrum $g_{w}\left(k_{r} ; \hat{r}, z, z_{s}\right)$ is thus defined below to obtain the local character of the wavenumber spectrum $g\left(k_{r}\right)$ in the asymptotic Hankel transform in Eq.(3-20):

$$
g_{w}\left(k_{r}, \hat{r}, z, z_{0}\right) \sim \frac{e^{i \frac{\pi}{4}}}{\sqrt{2 \pi k_{r}}} \int_{-\infty}^{\infty} w_{L}(r ; \hat{r}) p\left(r, z, z_{0}\right) e^{-i k, r} \sqrt{r} d r
$$

The range-varying peaks in $\sqrt{k_{r}}\left|g_{w}\left(k_{r} ; \hat{r}, z, z_{0}\right)\right|$ then reflect the modal evolution of the waveguide. These peak positions, however, do not exactly correspond to the local eigenvalues in the range-dependent case because the eigenvalues do not remain constant over the range covered by the short window. Shortening the window length further to localize their behavior gives rise to another type of error when evaluating Eq.(3-30). This error originates from the so-called "uncertainty principle," in which both wavenumber and range cannot be determined with arbitrary precision $[47,71,72]$.

In order to evaluate quantitatively the output of the above STFT, let us utilize the pressure field expressed by Eq.(2-12) in Chapter 2.2, which is derived from adiabatic mode theory with the assumption of a gradually changing medium in the lateral direction.

Before proceeding, a remark should be made on the choice of the coordinate system. Namely, in a range-dependent medium, it is essential to choose a coordinate system fixed 

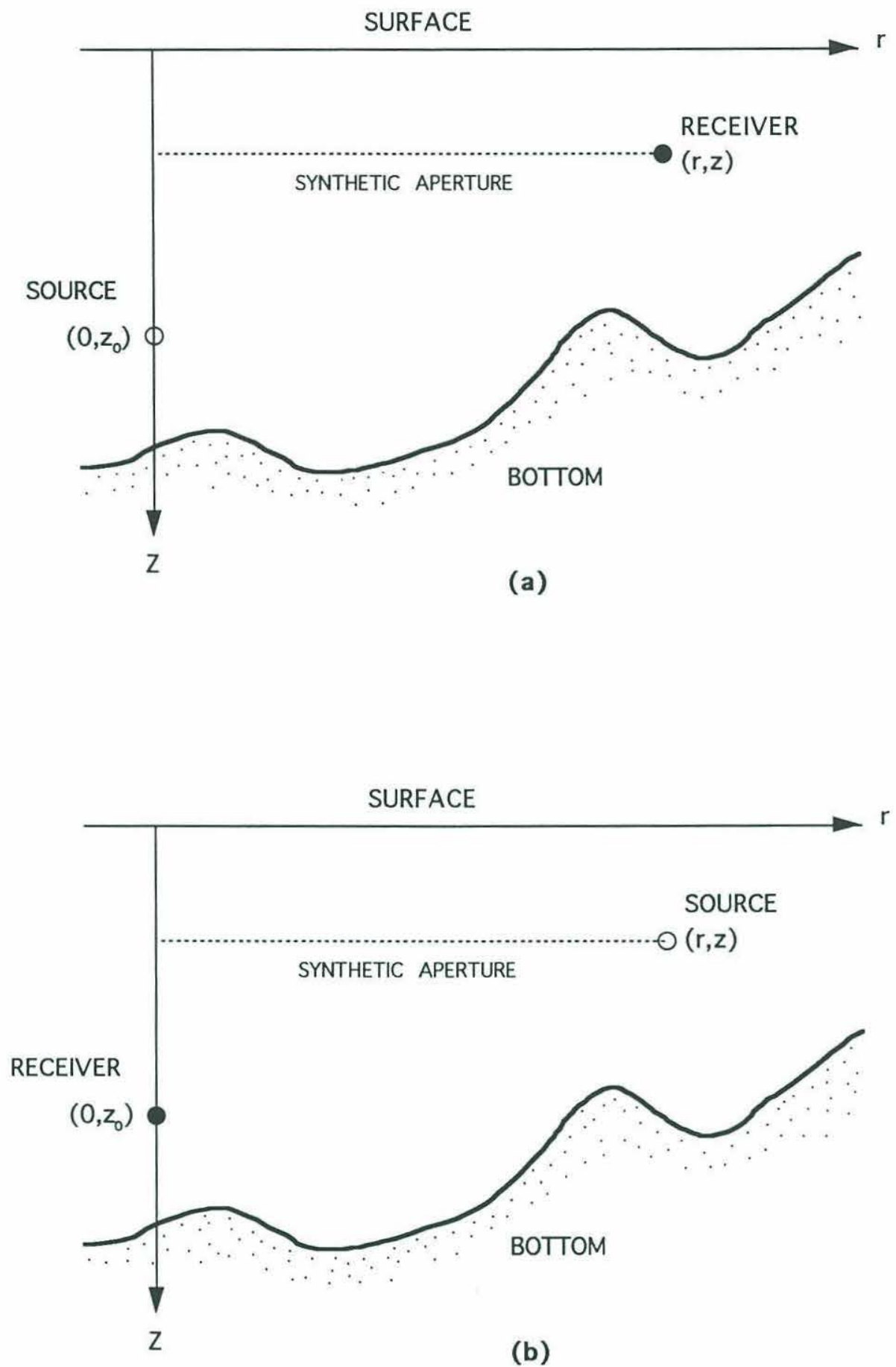

Figure 3-1: Geometry of the reciprocity relation between source and receiver positions. (a) Source and (b) receiver are fixed in space. 
in space for describing both the pressure field and the environmental parameters as a function of position. In Figure 3-1(a), given a fixed point source, the $z$ axis is chosen so as to pass through the source position $\left(0, z_{0}\right)$. The pressure field due to this source, $p\left(r, z, z_{0}\right)$, can be measured by the receiver placed at $(r, z)$. In contrast, if the receiver is fixed and the point source changes its position, then the $z$ axis is chosen so as to pass through the receiver position $\left(0, z_{0}\right)$. By virtue of the reciprocity principle, the pressure due to the source at $(r, z)$ which is measured by the receiver at $\left(0, z_{0}\right)$ in Figure $3-1(b)$ is identical to the pressure due to the source at $\left(0, z_{0}\right)$ which is measured by the receiver at $(r, z)$ in Figure 3-1(a). Therefore, in either case, we can use Eq.(2-12) by choosing the coordinate system as shown in Figure 3-1.

Substitution of the pressure field $p\left(r, z, z_{0}\right)$ in Eq.(2-12) into Eq.(3-30), followed by a change of the order of Fourier integration and mode summation, yields

$$
g_{w}\left(k_{r} ; \hat{r}, z, z_{0}\right) \sim \frac{i}{\sqrt{k_{r}}} \sum_{n} \int_{-\infty} w_{L}(r ; \hat{r}) \frac{u_{n}\left(0, z_{0}\right) u_{n}(r, z)}{\sqrt{\kappa_{n}(r)}} e^{i \int_{0}^{\prime} x_{n}(r) d r} e^{-i k_{r} r} d r
$$

Alternatively, rewriting Eq.(3-31) with the use of a Fourier operator, $\mathfrak{S}\{\bullet\} \equiv \int r e^{-i k r}$, yields

$$
g_{w}\left(k_{r} ; \hat{r}, z, z_{0}\right) \sim \frac{i}{\sqrt{k_{r}}} \sum_{n} u_{n}\left(0, z_{0}\right) g_{n}\left(k_{r}, \hat{r}, z\right)
$$

with

$$
g_{n}\left(k_{r} ; \hat{r}, z\right)=\mathfrak{I}\left\{w_{L}(r ; \hat{r}) \frac{u_{n}(r, z)}{\sqrt{\kappa_{n}(r)}} e^{i \int_{0} \gamma_{n}(r) d r}\right\},
$$

where $g_{n}$ represents the spectrum associated with the $n$th mode. It is then immediately seen that the departure of the peak position in $\left|g_{n}\left(k_{r} ; \hat{r}, z, z_{s}\right)\right|$ from $\operatorname{Re}\left[\kappa_{n}(r)\right]$ originates from the following two factors: One is the range dependence of $g_{n}$; the other is the 
interference with the sidelobes of the adjacent mode $\left(g_{n \pm 1}\right)$. First, we will examine the peak shift due to the first factor; then in Section 3.3 we will examine the peak shift due to the second factor.

\subsubsection{Peak shift due to range dependence in the local eigenvalue}

In this subsection we will confine ourselves to the spectrum $g_{n}$ given by Eq.(3-33) and examine the effect of range dependence in the local eigenvalue on this spectrum.

Since the local eigenvalues vary slowly with range in the framework of adiabatic mode theory, the phase term in Eq.(3-33) may be expanded in a Taylor series around the center position $\hat{r}$ of the window. The expansion to the fourth order yields

$$
\int_{0}^{r} k_{n}(r) d r \approx \int_{0}^{\hat{k}} k_{n}(r) d r+k_{n}(\hat{r})(r-\hat{r})+\left.\frac{1}{2} \frac{d k_{n}}{d r}\right|_{\hat{r}}(r-\hat{r})^{2}+\left.\frac{1}{6} \frac{d^{2} k_{n}}{d r^{2}}\right|_{\hat{r}}(r-\hat{r})^{3},
$$

where $k_{n}(r)$ represents the real part of the local eigenvalue, i.e., $k_{n}(r) \equiv \operatorname{Re}\left[\kappa_{n}(r)\right]$, and this approximation is valid within the range $|r-\hat{r}| \ll<\left.4 \frac{d^{2} k_{n}}{d r^{2}}\right|_{\hat{r}}\left|\frac{d^{3} k_{n}}{d r^{3}}\right|_{\hat{r}}$.

Then substituting Eq.(3-34) into Eq.(3-33) along with a new variable $\tau \equiv r-\hat{r}$ yields

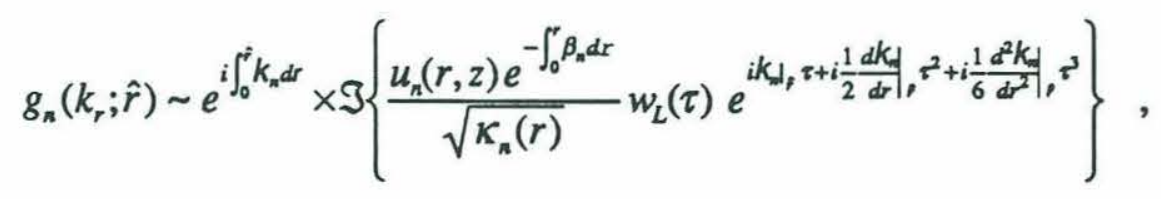

where the window function is assumed to be given as a function of $\tau$, i.e., $w_{L}(r, \hat{r})$ $=w_{L}(\tau)$. In executing the Fourier transform of the $\tau$-dependent functions, it is convenient to integrate with respect to $\tau$ rather than $r$. Hence, from the relation of $\tau \equiv r-\hat{r}$, we may replace $\mathfrak{S}\{\bullet\} \equiv \int d r e^{-i k, r}$ with $e^{-i k \hat{r}} \mathfrak{I}_{\tau}\{\bullet\}$, where $\mathfrak{I}_{\tau}\{\bullet\} \equiv \int d \tau e^{-i k \tau}$. Use of this replacement after deconvolution in Eq.(3-35) yields 


$$
\begin{aligned}
& g_{n}\left(k_{r} ; \hat{r}\right) \sim e^{i \int_{0}^{p} k_{n} d r} \\
& \times \mathfrak{S}\left\{\frac{u_{n}(r, z)}{\sqrt{\kappa_{n}(r)}}\right\} * \mathfrak{S}\left\{e^{-\int_{0}^{r} \beta_{n} d r}\right\} *\left[e^{-i k_{r}} \mathfrak{I}_{\tau}\left\{w_{L}(\tau) e^{i k_{n}, \tau+i \frac{1}{2} \frac{d k_{n}}{d r}, \tau^{2}+i \frac{1}{6} \frac{d^{2} k_{1}}{d r^{2}}, \tau^{3}}\right\}\right] .
\end{aligned}
$$

Since both $\kappa_{n}(r)$ and $u_{n}(r, z)$ vary slowly with range $r$ due to the adiabatic assumption, the spectrum $\mathfrak{I}\left\{u_{n} / \sqrt{\kappa_{n}}\right\}$ is mainly concentrated in a region that is extremely close to zero in wavenumber. Thus, this transform behaves in a manner similar to $\delta\left(k_{r}\right)$ and is not a dominant factor that causes the shift of the peak in Eq.(3-36). As for the second Fourier transform in Eq.(3-36), its major role is to broaden the peak as expected from the result in the horizontally stratified case, where $\beta_{n}(r)$ is constant. Thus the shift in peak position in $\left|g_{n}\right|$ is primarily influenced by the last transform in Eq.(3-36), which, for convenience, we denote by $F_{0}\left(k_{r}\right)$.

In order to evaluate $F_{0}\left(k_{r}\right)$, let us examine it by further decomposition as

$$
\begin{aligned}
F_{0}\left(k_{r}\right) & =\mathfrak{I}_{\tau}\left\{w_{L}(\tau) e^{i k_{1}\left|, \tau+i \frac{1}{2} \frac{d k_{j}}{d r}, \tau^{2}+i \frac{1}{6} \frac{d^{2} k_{k}}{d r^{2}}\right| \tau^{3}}\right\} \\
& =\mathfrak{I}_{\tau}\left\{w_{L}(\tau)\right\} * \mathfrak{I}_{\tau}\left\{e^{i k_{r}(\hat{r}) \tau}\right\} * \mathfrak{I}_{\tau}\left\{e^{i \frac{1}{2} k_{n}^{\prime}(\hat{r}) \tau^{2}}\right\} * \mathfrak{I}_{\tau}\left\{e^{i \frac{1}{\delta} k_{n}^{\prime \prime}(\hat{r}) \tau^{3}}\right\},
\end{aligned}
$$

where $\left.\frac{d k_{n}}{d r}\right|_{f} \equiv k_{n}^{\prime}(\hat{r})$ and $\left.\frac{d^{2} k_{n}}{d r^{2}}\right|_{f} \equiv k_{n}^{\prime \prime}(\hat{r})$. The Fourier transform of each member on the right hand side of Eq.(3-37) can then be evaluated in an analytical manner and these results are described below.

\section{The Fourier transform of each member in Eq. (3-37)}

1. The first transform $\mathfrak{I}_{\tau}\left\{w_{L}(\tau)\right\}$ in Eq.(3-37) takes on a functional form which depends on the type of window. For example, the rectangular window 


$$
w_{L}(\tau)=\left\{\begin{array}{cc}
\frac{1}{L} & (\tau \mid \leq L / 2) \\
0 & (\tau \mid>L / 2)
\end{array}\right.
$$

yields

$$
\mathfrak{I}_{\tau}\left\{w_{L}(\tau)\right\}=\operatorname{sinc}\left(\frac{L}{2} k_{r}\right)
$$

and the Hanning window

$$
w_{L}(\tau)=\left\{\begin{array}{cc}
\frac{2}{L} \cos ^{2}\left(\pi \frac{\tau}{L}\right) & (|\tau| \leq L / 2) \\
0 & (|\tau|>L / 2)
\end{array}\right.
$$

yields

$$
\mathfrak{I}_{\tau}\left\{w_{L}(\tau)\right\}=\operatorname{sinc}\left(\frac{L}{2} k_{r}\right)+\frac{1}{2}\left[\operatorname{sinc}\left(\frac{L}{2}\left(k_{r}-\frac{2 \pi}{L}\right)\right)+\operatorname{sinc}\left(\frac{L}{2}\left(k_{r}+\frac{2 \pi}{L}\right)\right)\right] \text {. }
$$

In Eq.(3-41) the first sidelobe can be decreased by $33 \mathrm{~dB}$ as compared to $13 \mathrm{~dB}$ in Eq.(339) at the cost of discarding some information in the field data by the Hanning taper. In other words, the field data close to the edge of the Hanning window are weighted weakly, while the field data at the center of the window are emphasized. This situation is, however, suitable to our purpose if we recall the fact that the local eigenvalue at the center of the window, $k_{n}(\hat{r})$, is the target we want to detect. Namely, our interest lies in the peak position of the range-varying spectrum rather than in its correct shape; therefore the Hanning window is preferable to the rectangular window in the current scheme.

2. The second Fourier transform $\mathfrak{I}_{\tau}\left\{e^{i k_{n}(\hat{r}) \tau}\right\}$ in Eq.(3-37) is immediately obtained from the definition of the Dirac delta function [47,70], yielding 


$$
\mathfrak{I}_{\tau}\left\{e^{i k(\hat{r}) \tau}\right\}=\delta\left(k_{r}-k_{n}(\hat{r})\right)
$$

We note that the local eigenvalue $k_{n}(\hat{r})$ is constant in Eq.(3-42) as long as $\hat{r}$ is fixed.

3. The third transform $\mathfrak{I}_{\tau}\left(e^{i \frac{1}{2} k_{n}^{\prime}(\hat{r}) \tau^{2}}\right\}$ in Eq.(3-37) can be rewritten as

$$
\begin{aligned}
& \Im_{\tau}\left\{e^{i \frac{1}{2} k_{n}^{\prime}(\hat{r}) \tau^{2}}\right\}=\int_{-\infty}^{+\infty} e^{i\left(\frac{1}{2} k_{n}(\hat{\gamma}) \tau^{2}-k, \tau\right)} d \tau
\end{aligned}
$$

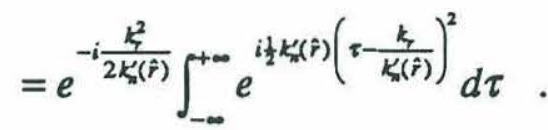

Use of the identity [57]

$$
\int_{-\infty}^{+\infty} e^{-x^{2}} d x=\sqrt{\pi}
$$

in Eq.(3-43) gives

$$
\mathfrak{S}_{\tau}\left\{e^{i \frac{i}{2} k_{n}^{\prime}(\hat{r}) \tau^{2}}\right\}=\sqrt{\frac{2 \pi}{\left|k_{n}^{\prime}(\hat{r})\right|}} e^{-i \frac{k_{\tau}^{2}}{2 k_{n}^{\prime}(\hat{r})}+i s_{g n}\left(k_{n}^{\prime}(\hat{r})\right) \frac{\pi}{4}},
$$

where sgn represents the sign of its argument.

4. The last transform $\mathfrak{I}_{\tau}\left\{e^{i \mathrm{\xi}_{n}^{*}(\hat{r}) \tau^{3}}\right\}$ in Eq.(3-37) may be rewritten as

$$
\begin{aligned}
& \mathfrak{I}_{\tau}\left\{e^{\left.i \frac{i}{6} k_{m} \hat{r}\right) \tau^{3}}\right\}=\int_{-\infty}^{+\infty} e^{i\left(\frac{k_{\pi}(\hat{r}) \tau^{3}-k, \tau}{6}\right)} d \tau \\
& =\int_{-\infty}^{+\infty} e^{-i\left[\frac{v^{3}}{3}+\frac{k_{r}}{\left(-\frac{1}{2} k_{n}^{\prime \prime}(\hat{r})\right)^{\frac{3}{}}} v\right]} \frac{d v}{\left(-\frac{1}{2} k_{n}^{\prime \prime}(\hat{r})\right)^{\frac{3}{3}}},
\end{aligned}
$$


where the integration variable $\tau$ has been changed to $v$ as $\tau=v /\left(-\frac{1}{2} k_{n}^{\prime \prime}(\hat{r})\right)^{\frac{1}{3}}$. Here we notice that the integral in Eq.(3-46) is equivalent to the integral form defining the Airy function [57]:

$$
A i(x) \equiv \frac{1}{2 \pi} \int_{-\infty}^{+\infty} e^{-i\left(\frac{1}{3} v^{3}+x v\right)} d v
$$

Thus we can represent the integral in Eq.(3-46) as $2 \pi A i(x)$ with replacement of $x=k_{r} /\left(-\frac{1}{2} k_{n}^{\prime \prime}(\hat{r})\right)^{\frac{1}{3}}$, yielding

$$
\mathfrak{I}_{\tau}\left\{e^{i \frac{1}{6} k_{m}^{\prime \prime}(\hat{r}) \tau^{3}}\right\}=\frac{2 \pi}{\left(-\frac{1}{2} k_{n}^{\prime \prime}(\hat{r})\right)^{\frac{1}{3}}} A i\left(\frac{k_{r}}{\left(-\frac{1}{2} k_{n}^{\prime \prime}(\hat{r})\right)^{\frac{1}{3}}}\right) .
$$

The results of our analysis of the terms in Items 1-4 indicate that the peak in $\left|F_{0}\left(k_{r}\right)\right|$ forms near $k_{r}=k_{n}(\hat{r})$, primarily due to Eq. $(3-42)$ and the rest of the terms serve to shift the peak position or broaden the peak shape in the spectrum as explained below. In order to determine the departure from $k_{n}(\hat{r})$, the convolutions in Eq.(3-37) must be executed.

Convolution of each member in Eq.(3-37)

1. Convolution of $\mathfrak{I}_{\tau}\left\{w_{L}(\tau)\right\}$ and $\mathfrak{I}_{\tau}\left\{e^{i \frac{1}{6} k_{n}^{* \prime}(\hat{r}) \tau^{3}}\right\}$

First, we will convolve $\mathfrak{I}_{\tau}\left\{w_{L}(\tau)\right\}$ and $\mathfrak{I}_{\tau}\left\{e^{i \frac{1}{\delta} k_{n}^{n}(\hat{r}) \tau^{3}}\right\}$ in Eq.(3-37). As shown above, $\mathfrak{I}_{\tau}\left\{w_{L}(\tau)\right\}$ for the rectangular and Hanning windows are given by Eq.(3-39) and Eq.(3-41), respectively; $\mathfrak{I}_{\tau}\left\{e^{i \frac{1}{6} k_{n}^{\prime \prime}(\hat{r}) \tau^{3}}\right\}$ is given by Eq.(3-48) using the Airy function. To facilitate the expression, let $\mathfrak{I}_{\tau}\left\{w_{L}(\tau)\right\}$ be denoted by $W\left(L k_{r}\right)$, in which the window length $L$ plays the role of making the argument dimensionless, as seen in Eq.(3-39) and Eq.(3-41). In a similar fashion, $\left(-\frac{1}{2} k_{n}^{\prime \prime}(\hat{r})\right)^{-\frac{1}{3}}$ in Eq.(3-48) has the dimension of length, so that denoting it as $\ell$ represents the right-hand side of this equation as $2 \pi \ell A i\left(\ell k_{r}\right)$. Thus, it follows that

$$
F_{1}\left(k_{r}\right)=2 \pi \ell A i\left(\ell k_{r}\right) * W\left(L k_{r}\right)
$$


or, alternatively, using the nondimensional parameters $\varsigma \equiv \ell k_{r}$ and $\varepsilon \equiv L / \ell$,

$$
F_{1}(\varsigma)=2 \pi \ell A i(\varsigma) * W(\varepsilon \varsigma)
$$

Figure 3-2 shows the results of $F_{1}(\varsigma)$ for a set of positive $\varepsilon$, which are obtained by numerically executing the convolution in Eq.(3-50); the Hanning window given by Eq.(3$41)$ is here used for $W\left(L k_{r}\right)$. In this figure, $F_{1}(\varsigma)$ are normalized so that their maximum values take one. Note that, from the definitions $\ell \equiv\left(-\frac{1}{2} k_{n}^{\prime \prime}(\hat{r})\right)^{-t}$ and $\varepsilon \equiv L / \ell$, the positive $\varepsilon$ corresponds to a negative $k_{n}^{\prime \prime}(\hat{r})$. If $\varepsilon<0$, that is, $k_{n}^{\prime \prime}(\hat{r})>0$, then we may simply obtain a symmetric result about the ordinate in Figure 3-2.

As seen from comparison of the results in Figure 3-2, the peak position shifts from 0 to -1 when $|\varepsilon|$ takes larger values, which correspond to $|\ell|<L$ or, equivalently, $\left|k_{n}^{\prime \prime}(\hat{r})\right|>2 / L^{3}$. This is because the substantial contribution to $F_{1}(\varsigma)$ by the convolution in Eq.(3-50) occurs around the range where $A i(\varsigma)$ takes a maximum, i.e., $\varsigma_{0} \approx-1\left(A i^{\prime}\left(\varsigma_{0}\right) \equiv 0\right)$, since $W(\varsigma)$ has a narrow width for a large $|\varepsilon|$ (the first null $=2 \pi / \varepsilon=2 \pi \ell / L$ ). Figure 3-3 shows an example for this case, i.e., $W(\varsigma)$ for $|\varepsilon|=16$, whose bandwidth is small compared to that of $A i(\varsigma)$ at $\varsigma_{0} \approx-1$. In an extreme case such as $|\varepsilon| \gg>1$ or $L\left|k_{n}^{\prime \prime}(\hat{r})\right|^{\frac{1}{3}} \gg>1$, the peak position in $F_{1}(\varsigma)$ is located at $\varsigma \approx-1$, that is, $k_{r} \approx-1 / \ell \approx\left(\frac{1}{2} k_{n}^{\prime \prime}(\hat{r})\right)^{\dagger}$. This value corresponds to the actual departure from $k_{n}(\hat{r})$ and the peak position shifts to the positive direction for $k_{n}^{\prime \prime}(\hat{r})>0$ and negative for $k_{n}^{\prime \prime}(\hat{r})<0$, respectively.

In contrast, it is seen from Figure 3-2 that for small $|\varepsilon|$, that is, $\left|k_{n}^{\prime \prime}(\hat{r})\right|<2 / L^{3}$, the peak position gets close to zero even though the peak width broadens. This is because the effective range in the convolution process becomes large, so that the contribution from the positive and negative loops in $A i(\varsigma)$ for $\varsigma<0$ tends to mutually cancel each other out. As an example of this case, $W(\varsigma)$ for $|\varepsilon|=1$ is compared in Figure 3-3 with $A i(\varsigma)$. 

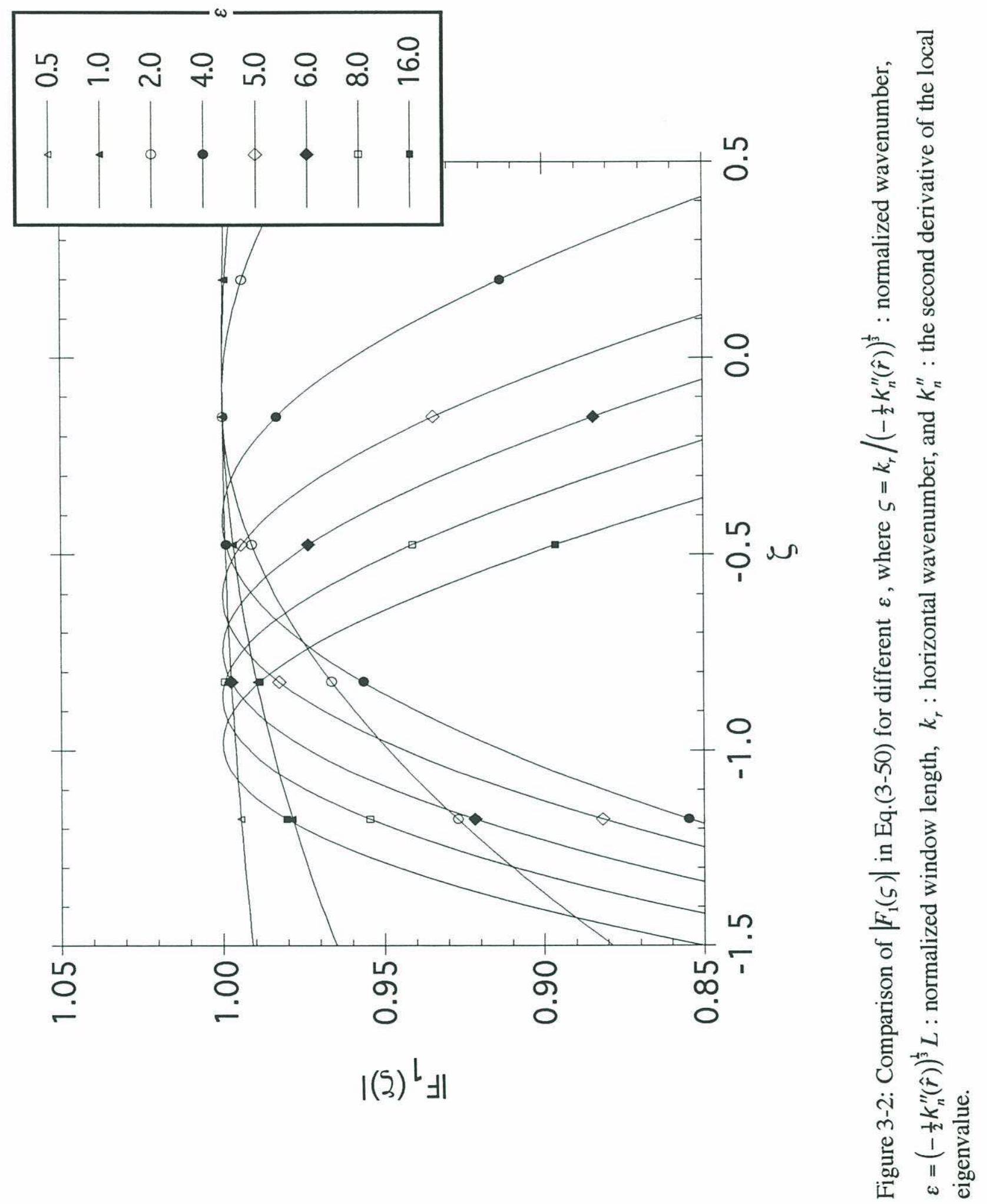


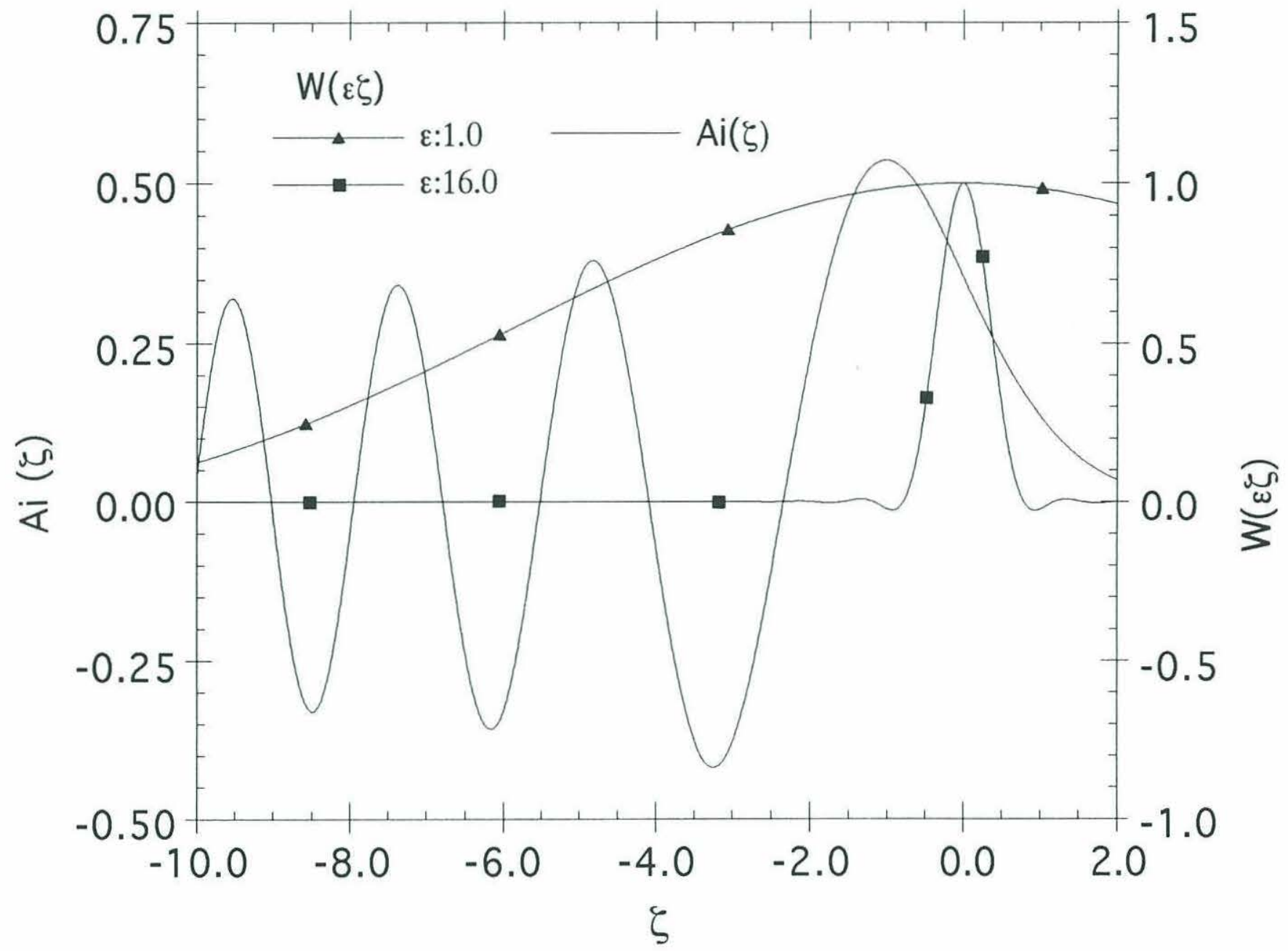

Figure 3-3: Airy function $A i(\varsigma)$ and Fourier transform of the Hanning window $W\left(L k_{r}\right) \equiv W(\varepsilon \varsigma)$ with different window lengths $(L)$. 


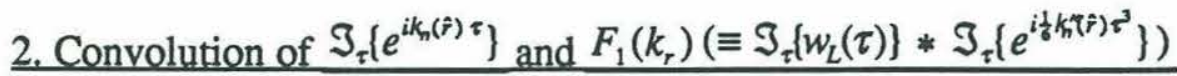

The next step for determining $F_{0}\left(k_{r}\right)$ in Eq.(3-37) is to take a convolution of $F_{1}\left(k_{r}\right)$ in Eq.(3-50) and $\mathfrak{I}_{\tau}\left\{e^{i k_{n}(\hat{r}) \tau}\right\}$, which is represented by $\delta\left(k_{r}-k_{n}(\hat{r})\right)$ as shown in Eq.(3-42). From the sifting property in the Delta function [47,70], it follows that

$$
F_{1}\left(k_{r}\right) * \delta\left(k_{r}-k_{n}(\hat{r})\right)=F_{1}\left(k_{r}-k_{n}(\hat{r})\right)
$$

Thus it is seen that $\varsigma=0$ in Figure 3-2 corresponds to $k_{r}=k_{n}(\hat{r})$. Therefore, as discussed above, the departure of the peak position from $k_{n}(\hat{r})$ depends on both the window length $L$ and $\left|k_{n}^{\prime \prime}(\hat{r})\right|$, and its shifting direction is determined from the sign of $k_{n}^{\prime \prime}(\hat{r})$.

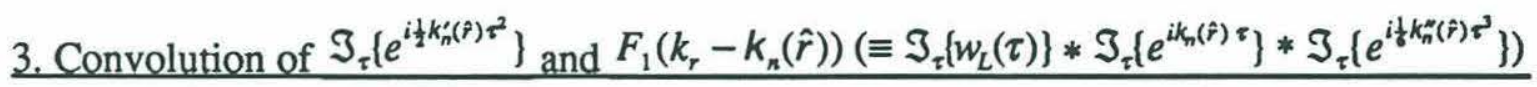

As a final step for obtaining $F_{0}\left(k_{r}\right)$, we have to take a convolution of $F_{1}\left(k_{r}-k_{n}(\hat{r})\right)$ in Eq.(3-50) and $\mathfrak{I}_{\tau}\left\{e^{i \frac{i}{2} k_{n}^{\prime}(P) \tau^{2}}\right\}$, which is evaluated in Eq.(3-45), giving

$$
F_{0}\left(k_{r}\right)=\frac{e^{i \operatorname{sgn} n\left(k_{n}^{\prime}(\hat{r})\right) \frac{\pi}{4}}}{\sqrt{\left|k_{n}^{\prime}(\hat{r})\right|}} \int_{-\infty}^{+\infty} F_{1}\left(\varsigma-k_{n}(\hat{r})\right) e^{-i \frac{\left(k_{r}-\zeta\right)^{2}}{2 k_{n}^{\prime}(\hat{r})}} d \zeta
$$

In order to evaluate the above integral, one may utilize the method of stationary phase $[70,73]$ such that

$$
f(x)=\int q(\varsigma) e^{i x \varphi(\varsigma)} d \varsigma \underset{|x|>>1}{\longrightarrow} \sqrt{\frac{2 \pi}{x\left|\varphi^{\prime \prime}\left(\zeta_{s}\right)\right|}} q\left(\zeta_{s}\right) e^{i\left[x \varphi\left(\zeta_{s}\right)+s_{g n}\left(\varphi^{\prime \prime}\left(\zeta_{s}\right)\right) \frac{\pi}{4}\right]},
$$

where $\zeta_{s}$ is a stationary point which satisfies $\varphi^{\prime}\left(\zeta_{s}\right) \equiv 0$. Accordingly, for Eq.(3-52) we can set $x=1 / k_{n}(\hat{r}), q(\varsigma)=F_{1}\left(\varsigma-k_{n}(\hat{r})\right)$, and $\varphi(\varsigma)=\frac{1}{2}\left(\varsigma-k_{r}\right)^{2}$. Since $\left|k_{n}^{\prime}(\hat{r})\right|$ is small within the framework of the adiabatic approximation, $|x| \gg>1$ is satisfied. It is also found from $\varphi^{\prime}\left(\zeta_{s}\right)=\zeta_{s}-k_{r}$ that $\zeta_{s}=k_{r}$. Hence, we can finally obtain 


$$
F_{0}\left(k_{r}\right) \approx e^{i \operatorname{sgn}\left(k_{n}^{\prime}(\hat{r})\right) \frac{\pi}{4}} F_{1}\left(k_{r}-k_{n}(\hat{r})\right)
$$

As a closing remark, the spectrum amplitude $\left|g_{n}\left(k_{r} ; \hat{r}\right)\right|$, that is proportional to $\left|F_{0}\left(k_{r}\right)\right|$, has a peak shifted from $k_{n}(\hat{r})$, and its departure is primarily determined by the relationship between the window length $L$ and the second derivative of the local eigenvalue $k_{n}^{\prime \prime}(\hat{r})$. As $L\left|k_{n}^{\prime \prime}(\hat{r})\right|^{\dagger}$ increases, the departure from $k_{n}(\hat{r})$ becomes larger. Depending upon whether $k_{n}^{\prime \prime}(\hat{r})$ is positive or negative, the peak is shifted in the positive or negative direction in the wavenumber $k_{r}$, respectively. For $L\left|k_{n}^{\prime \prime}(\hat{r})\right|^{\$} \gg>1$, the shift distance approaches $\left|k_{n}^{\prime \prime}(\hat{r}) / 2\right|^{\dagger}$. For $\left|k_{n}^{\prime \prime}(\hat{r})\right| \rightarrow 0$, the peak position approaches $k_{n}(\hat{r})$.

\subsubsection{Analysis of simulated data}

By taking a simple example of a single mode situation, let us numerically demonstrate that the departure of the estimated peak position from the local eigenvalue is related to the second derivative of the local eigenvalue and the length of the sliding window, as discussed above.

In order to facilitate the numerical analysis, we will initially specify a local eigenvalue $k_{n}(r)$ as a function of range rather than set up an ocean environment to provide $k_{n}(r)$, which requires solving Eq. (2-8) at each range. Thus we can specify the values of $k_{n}(r)$ and compute $k_{n}^{\prime \prime}(r)$ whether or not there actually exists a waveguide for accommodating this mode. The solid line in Figure 3-4 is chosen for $k_{n}(r)$ so that it has a variable $k_{n}^{\prime \prime}(r)$ with range. At $900 \mathrm{~m}$ and $1100 \mathrm{~m}, k_{n}^{\prime \prime}(r)$ has its greatest magnitude but opposite sign. Between these points, $k_{n}(r)$ changes almost linearly with range and $k_{n}^{\prime}(r)$ has its maximum value in this range. In providing these values for $k_{n}(r)$, the cubic spline was utilized, so that the continuity of this curve is ensured up to the second derivative $k_{n}^{\prime \prime}(r)$. The factor $\left(\frac{1}{2} k_{n}^{\prime \prime}(r)\right)^{\dagger}$, discussed in Section 3.2.1, can then be 
calculated; its absolute value is shown in Figure 3-4 with a different scale indicated on the right side of the figure.

Now, in order to obtain the shift of the peak position in the spectrum $g_{n}\left(k_{r} ; z\right)$ in Eq.(3-33), the pressure field $p(r)$ has to be constructed by using the $k_{n}(r)$ specified in Figure 3-4. As seen from Eq.(2-12), the phase term is calculated simply from $\int_{0}^{r} k_{n}\left(r^{\prime}\right) d r^{\prime}$, whereas the amplitude requires the computation of the local eigenfunction $u_{n}(r)$. Since the effect of the range-dependent variation in eigenfunctions is small when compared to the phase variation, we may fix $u_{n}(r)$ to be a constant $C$. As a result, the pressure field is represented in the form of a WKBJ solution as $p(r)=C^{2} e^{i \int_{0} k_{n} d r} / \sqrt{k_{n} r}$.

Then in Eq.(3-33) we can numerically compute the STFT of this $p(r)$ by using the FFT. Figure 3-5(a) shows the range evolution of this output spectrum when using a Hanning window with a $400 \mathrm{~m}$ length and shifting its center every $20 \mathrm{~m}$. Figure 3-5(b) also shows the result obtained by using an $800 \mathrm{~m}$ length Hanning window; here the spectrum at about $1000 \mathrm{~m}$ demonstrates that the STFT processing does not track $k_{n}(r)$ well due to its strong variation over the range covered by the $800 \mathrm{~m}$ length window.

In Figure 3-6 the trajectory of the peak in the output spectrum in Figure 3-5(a) is compared with the exact local eigenvalue $k_{n}(r)$ along with the trajectories for different window lengths. The departure of these peaks from the exact value $k_{n}(r)$ is greatest at about $900 \mathrm{~m}$ and $1100 \mathrm{~m}$, where $\left|\frac{1}{2} k_{n}^{\prime \prime}(r)\right|^{\dagger}$ has its largest value. In addition, Figure 3-6 shows that the longer the window length, the larger the departure from $k_{n}(r)$. For comparison, these departures and $\left(\frac{1}{2} k_{n}^{\prime \prime}(r)\right)^{\frac{t}{t}}$ are shown together in Figure 3-7. This figure indicates that the direction of departure coincides with the sign of $k_{n}^{\prime \prime}(r)$, as discussed previously.

In order to extract local eigenvalues in a laterally varying waveguide, the asymptotic Hankel transform with a sliding window is applied to the range-dependent pressure field. The error in the eigenvalue estimation can be studied by applying the transform to the 


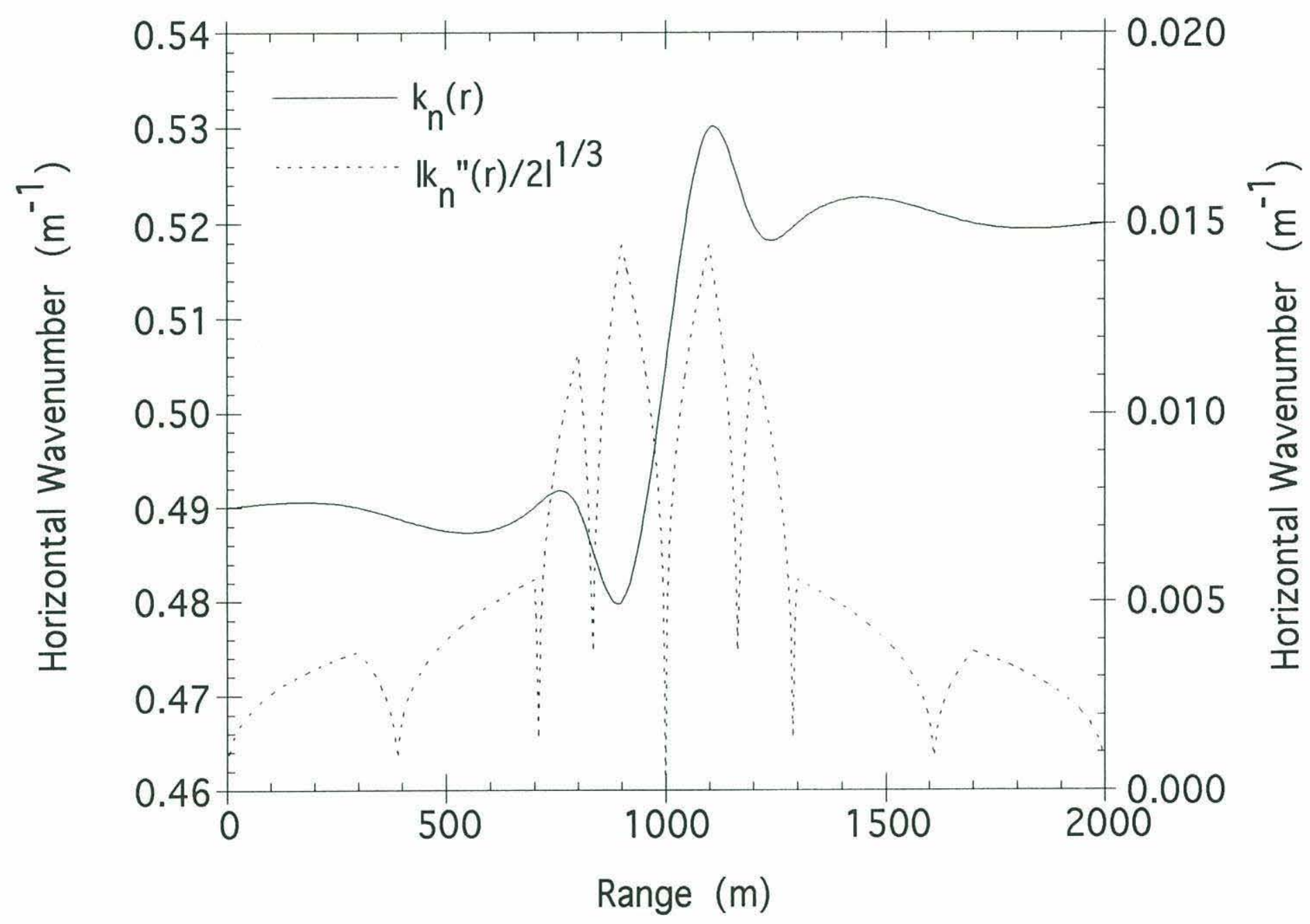

Figure 3-4: Local eigenvalue $k_{n}(r) ;\left|\frac{1}{2} k_{n}^{\prime \prime}(r)\right|^{1 / 3}$ is also shown with a different scale indicated on the right side of the figure. 


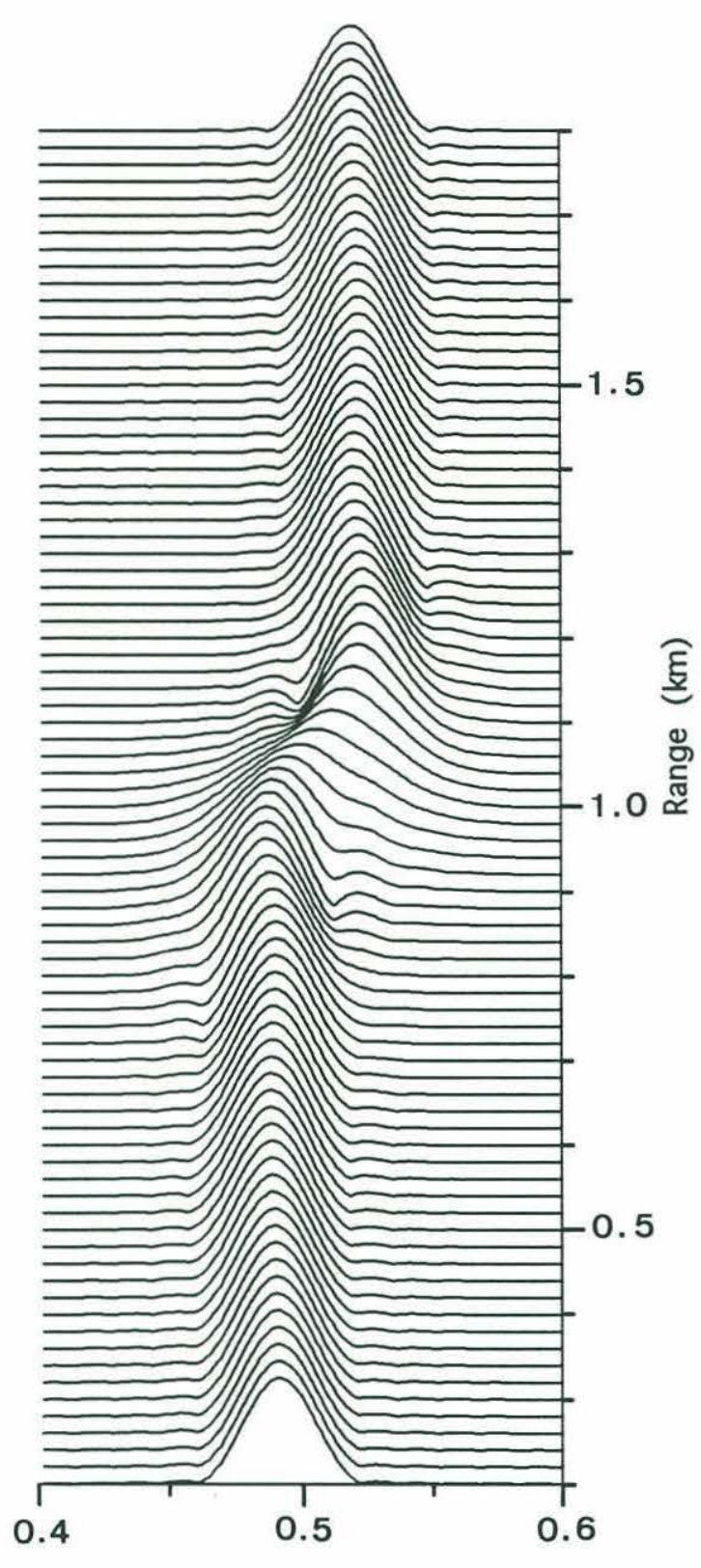

Horizontal Wavenumber $\left(\mathrm{m}^{-1}\right)$

(a)

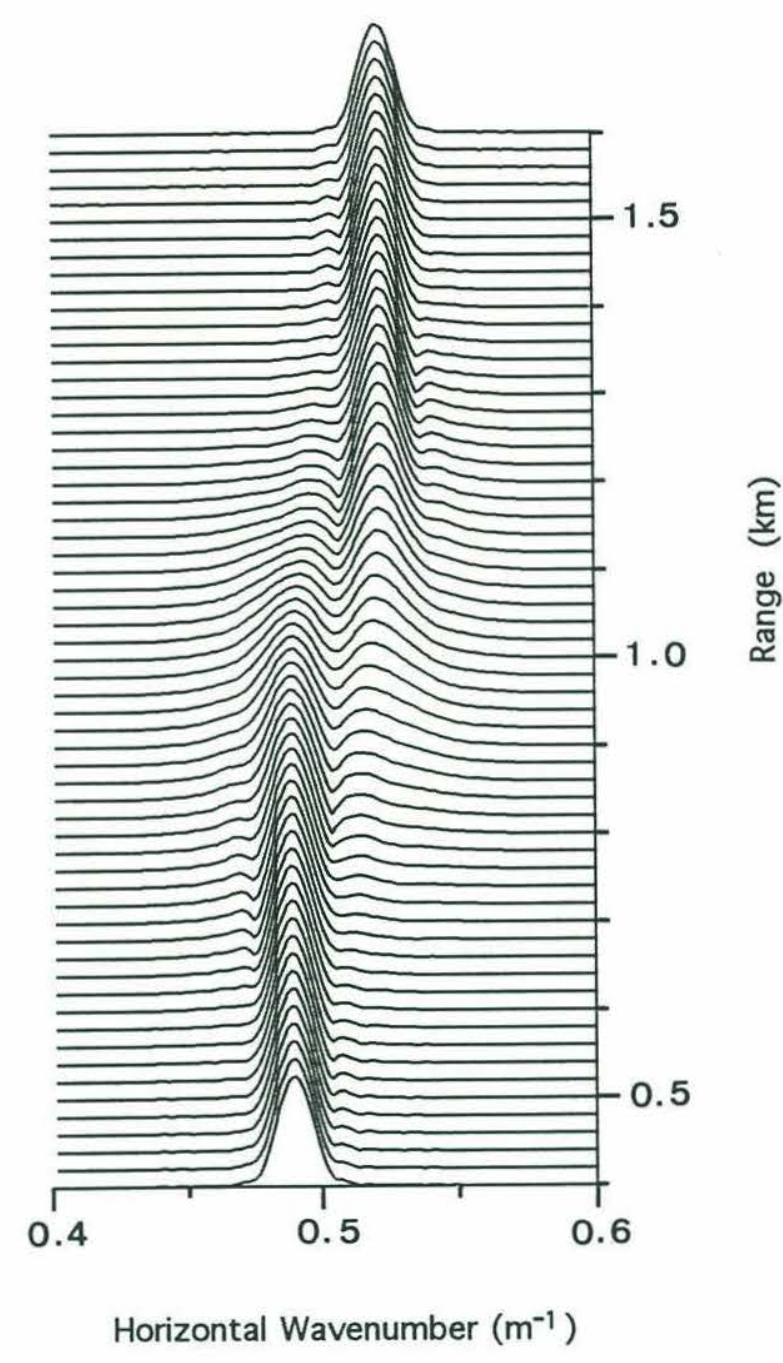

Horizontal Wavenumber $\left(\mathrm{m}^{-1}\right)$

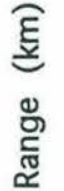

Figure 3-5: Range-dependent evolution of the output spectrum obtained by applying the STFT with a sliding Hanning window to a laterally inhomogeneous pressure field, which is simulated by using a single local eigenvalue shown in Figure 3-4: (a) the window length $=400 \mathrm{~m}$ and (b) the window length=800 $\mathrm{m}$. 


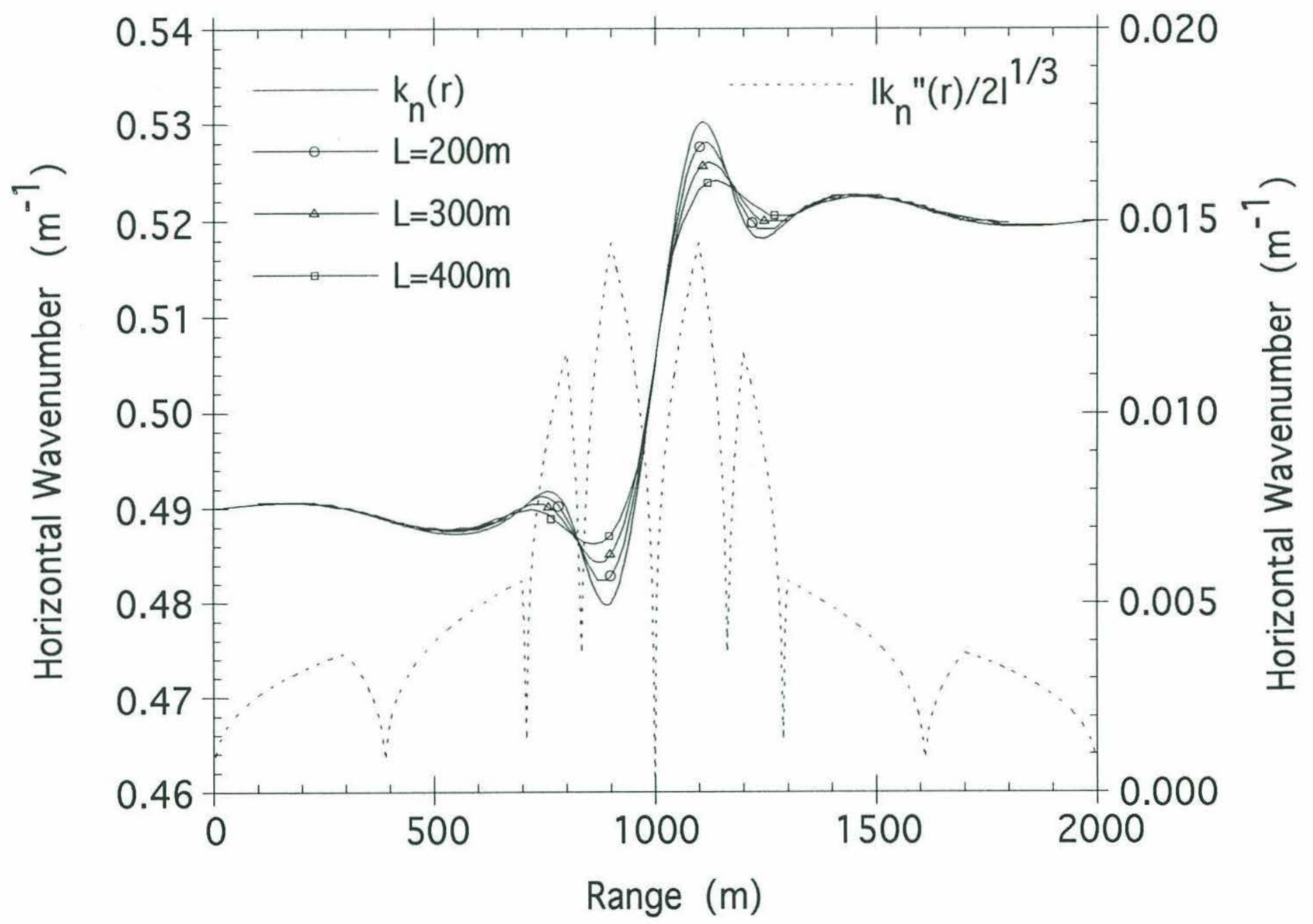

Figure 3-6: Comparison of the local eigenvalue $k_{n}(r)$ and peak trajectories in the STFT output spectrum obtained by applying different length $(L)$ Hanning windows to a laterally inhomogeneous pressure field, which is simulated by using a single local eigenvalue shown in Figure 3-4; $\left|\frac{1}{2} k_{n}^{\prime \prime}(r)\right|^{1 / 3}$ is also shown with a different scale indicated on the right side of this figure. 


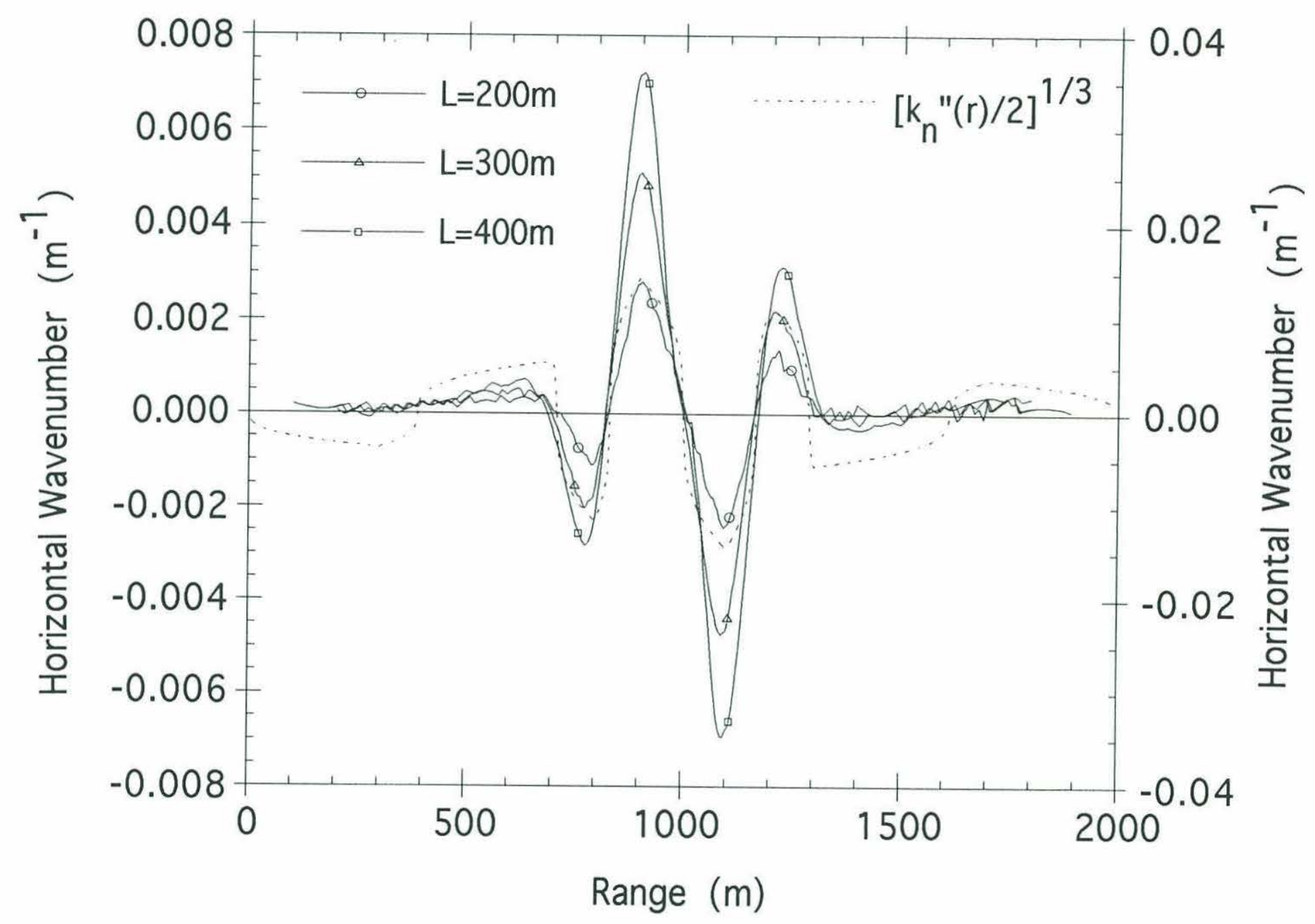

Figure 3-7: Difference of local eigenvalue $k_{n}(r)$ and modal peak trajectories in STFT output spectrum obtained by applying different length $(L)$ Hanning windows to a laterally inhomogeneous pressure field, which is simulated by using a single local eigenvalue shown in Figure 3-4; $\left(\frac{1}{2} k_{n}^{\prime \prime}(r)\right)^{\frac{1}{3}}$ is also shown with a different scale indicated on the right side of this figure. 
adiabatic mode field. For a single mode situation, a numerical analysis confirms that the degree of the departure of the peak position from the exact local eigenvalue is proportional to $L\left|k_{n}^{\prime \prime}(\hat{r})\right|^{\$}$. Multi-mode effects associated with the estimation error in this transform will be dealt with in Section 3.3.

\subsection{Application of mode filtering}

In multi-mode environments, the interference with the sidelobes of adjacent modes may induce another type of peak shift in the spectrum in addition to the shift due to the range-dependence of the local eigenvalue as discussed in Section 3.2. This kind of shift can be reduced by choosing a proper type and length of window, which depends on the difference in the eigenvalues for two adjacent modes.

If the distance of the adjacent modes in the wavenumber domain, however, is too small, then even an optimal window cannot separate two adjacent peaks in the spectrum. To overcome this difficulty, the separation of modes prior to the STFT is desirable. This separation can be realized by using mode filtering applied to data obtained on a fixed vertical array of receivers, if the environment at the array position is known a priori $[74,75]$. With the use of a coordinate system such that the $z$ axis lies along the vertical array (see Figure 3-8), the position of the $j$ th receiver $(1 \leq j \leq J)$ is described by $\left(0, z_{j}\right)$, and the pressure due to a point source at $(r, z)$ and measured by the $j$ th receiver is defined as $p_{j}$. Owing to the reciprocity principle, as discussed in Section 3.2, each $p_{j}$ is theoretically identical to the pressure $p\left(r, z, z_{j}\right)$ which is generated by a single point source at $\left(0, z_{j}\right)$, and so Eq.(2-12) for the adiabatic mode sum can be rewritten for a set of observations $p_{j}$ as

$$
p_{j}=\sum_{n}^{N} u_{j n} q_{n}(r, z) \quad(j=1, \ldots, J)
$$




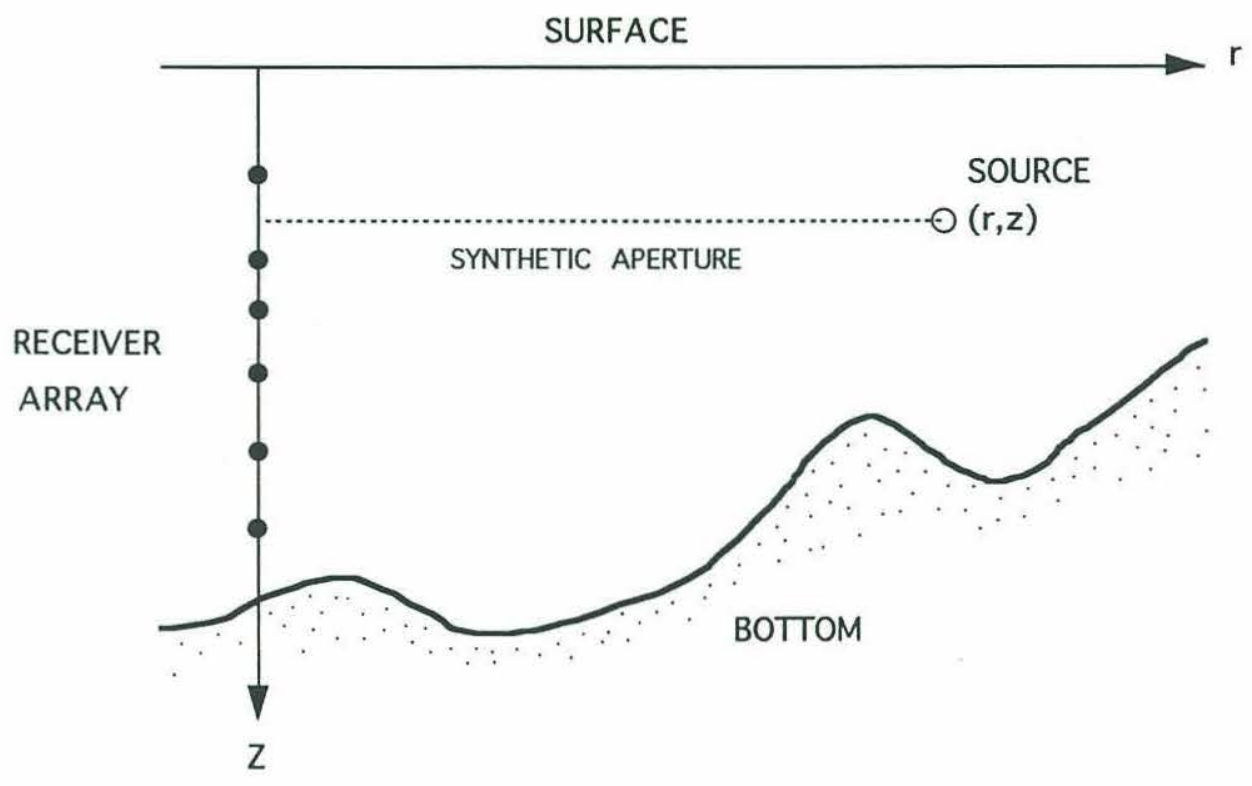

Figure 3-8: Geometry of a fixed vertical array of receivers and moving source with a constant depth. 
with

$$
\begin{aligned}
& u_{j n}=u_{n}\left(0, z_{j}\right), \\
& q_{n}(r, z)=i \sqrt{2 \pi} e^{-i \frac{\pi}{4}} \frac{u_{n}(r, z)}{\sqrt{\kappa_{n}(r) r}} e^{i \int_{0}^{r} k_{N}(r) d r} .
\end{aligned}
$$

As seen from this definition, each $q_{n}(r, z)$ is a range-dependent quantity associated with an individual mode. On the other hand, $u_{j n}$ is a range-independent coefficient obtained by solving the depth equation for the local eigenfunction, Eq.(2-8), with use of the known environment at the array site. If the number of receivers is equal to or more than the number of trapped modes, i.e., $J \geq N$, then Eq.(3-55) can be inverted as described below, and $q_{n}(r, z)$ can be expressed in terms of both the measured pressures $p_{j}$ and calculated eigenfunctions $u_{j n}$, thereby representing the signal carried by individual modes. Eq.(355) can be rewritten in matrix form as

$$
\mathbf{p}=\mathbf{U q}
$$

in which $\mathbf{p}_{j}=\left(p_{j}\right)$ and $\mathbf{q}_{n}=\left(q_{n}\right)$ are vectors with $J$ and $N$ elements, respectively, and $\mathbf{U}_{j n}=\left[u_{j n}\right]$ is a $J \times N$ matrix. If the vertical array is constructed of many receivers, thus enabling us to sample the field densely enough to write

$$
\int \frac{1}{\rho(z)} u_{n}(0, z) u_{m}(0, z) d z \approx \sum_{j=1}^{J} \frac{1}{\rho\left(z_{j}\right)} u_{n}\left(0, z_{j}\right) u_{m}\left(0, z_{j}\right)
$$

then it follows from the orthonormality condition for the local modes in Eq.(2-9) that

$$
\sum_{j=1}^{J} \frac{1}{\rho\left(z_{j}\right)} u_{n}\left(0, z_{j}\right) u_{m}\left(0, z_{j}\right) \approx \delta_{n m}
$$


or, equivalently, in matrix form

$$
\mathbf{V}^{t} \mathbf{U} \approx \mathbf{I}_{N}
$$

where $\mathbf{I}_{N}$ is an $N \times N$ identity matrix and $\mathbf{V}_{j n}=\left[u_{n}\left(0, z_{j}\right) / \rho\left(z_{j}\right)\right]$ is a $J \times N$ matrix. This matrix is also expressed using $\mathbf{U}$ as

$$
\mathbf{V}=\mathbf{R} \mathbf{U},
$$

where $\mathbf{R}$ is a $J \times J$ diagonal matrix

$$
\mathbf{R} \equiv\left[\begin{array}{ccc}
\frac{1}{\rho\left(z_{1}\right)} & & 0 \\
& \ddots & \\
0 & & \frac{1}{\rho\left(z_{J}\right)}
\end{array}\right] .
$$

Keeping the relation in Eq.(3-60) in mind, we can apply the so-called generalized inverse to Eq.(3-58). Namely, by multiplying both sides of Eq.(3-58) by the transpose matrix of $\mathbf{V}$, that is, $\mathbf{V}^{t}$, followed by further multiplication by $\left(\mathbf{V}^{t} \mathbf{U}\right)^{-1}$, we can obtain the desired result:

$$
\mathbf{q}_{I}=\left(\mathbf{V}^{t} \mathbf{U}\right)^{-1} \mathbf{V}^{t} \mathbf{p}
$$

Therefore, $q_{n}(r, z)$ can replace $p\left(r, z, z_{0}\right)$ in the asymptotic Hankel transform with the sliding window (Eq.(3-30)) in order to obtain the wavenumber spectrum for the $n$th local mode, yielding

$$
g_{w}\left(k_{r} ; \hat{r}, z\right) \sim \frac{e^{i \frac{\pi}{4}}}{\sqrt{2 \pi k_{r}}} \int_{-\infty}^{\infty} w_{L}(r ; \hat{r}) \sqrt{r} q_{n}(r, z) e^{-i k, r} d r
$$


or, equivalently, using Eq.(3-57):

$$
g_{w}\left(k_{r} ; \hat{r}, z, z_{s}\right) \sim \frac{i}{\sqrt{k_{r}}} \int_{-\infty} w_{L}(r ; \hat{r}) \frac{u_{n}(r, z)}{\sqrt{\kappa_{n}(r)}} e^{i \int_{0}^{\prime} k_{n}(r) d r} e^{-i k, r} d r
$$

From the peaks of $\sqrt{k_{r}}\left|g_{w}\right|$ obtained above, we can determine the local eigenvalues with the precision discussed in Section 3.2.

We recall, however, that the assumption of knowledge about the environment at the array position was made. In general, the sound speed in the water column as well as the water depth are readily measurable, but the bottom geoacoustic data are not. If there exists an isobath in some direction near the array site and also the bottom properties are constant over this range, then the bottom environment could be obtained by applying the inverse method, which was developed for the range-independent case, to the pressure measured over this range. For example, for a wedge-type environment, the water depth is constant in the direction perpendicular to the sloping bottom. As will be shown in Chapter 4, due to the effect of horizontal refraction, the pressure along this direction is not completely equivalent to that in a horizontally stratified medium with the same water depth, or more precisely, the same bottom environment. Practically, however, this effect will be negligible, because our interest here lies only in the bottom at the array site, and so the pressure field required for this inversion is limited to ranges relatively close to the source (but $\kappa_{n} r \gg>1$ ). This problem will be discussed again in Chapter 5 .

In deriving Eq.(3-64), a conventional least-mean-square (LMS) method has been applied to obtain $\left(\mathbf{V}^{t} \mathbf{U}\right)^{-1}$. When $\mathbf{V}^{t} \mathbf{U}$, however, is close to being a singular matrix such that the eigenvalues of this matrix are near zero, its inverse becomes unstable and, consequently, the output of Eq.(3-65) becomes affected by this processing error. As inferred from the approximation in Eq.(3-61), this problem is associated with the following two factors: One is the shape of the modes of interest and the other is the vertical array 
configuration, i.e., the total number and positions of the receivers. The former factor is characterized by the acoustic frequency and the depth-directional variation of the medium at the array site. In shallow water, the turning point depth of the ray associated with the maximum mode, which is often located in bottom sediment, would be of the most concern in connection with the lowest receiver. On the other hand, the latter factor is associated with the experimental design and, in general, the array spans only the water column or at best covers a few wavelengths below the bottom surface. Depending on the combination of these two factors, the above matrix $\mathbf{V}^{t} \mathbf{U}$ has a possibility of singularity, which leads to the unreliable output of the mode filter.

To overcome this problem, several improved methods are available. Yang used an eigenvector decomposition method (EDM) to estimate source location in the deep ocean in the North Arctic Sea [76]. It was demonstrated that this method can resolve more modes than the conventional least-mean-square (LMS) method. In EDM, one may discard the eigenvectors whose eigenvalues are smaller than a given threshold, whereas the stabilized least-mean-square (SLMS) method keeps those small eigenvalues by adding some small number such as one tenth of the trace of $\mathbf{V}^{t} \mathbf{U}$ to the diagonal components of this matrix $[48,49]$. The latter approach is one of the regularization methods for inverting a singular matrix. By employing these methods, we can improve the operation for obtaining $\mathbf{q}_{I}$ in Eq.(3-64) and expect reliable separation of the modes. Here we will describe these methods by using the well-known, singular value decomposition (SVD) method.

As proved in Ref. 43, the SVD method can decompose a $J \times N$ matrix $\mathbf{U}$ in the following form:

$$
\mathbf{U}=\mathbf{A}_{I} \Lambda_{I} \mathbf{B}_{I}^{t}
$$

with 


$$
\begin{array}{ll}
\Lambda_{I}=\left[\begin{array}{lll}
\lambda_{1} & & 0 \\
& \ddots & \\
0 & & \lambda_{I}
\end{array}\right] \quad\left(\lambda_{1}, \ldots, \lambda_{I}>0\right), \\
\mathbf{A}_{I}^{t} \mathbf{A}_{I}=\mathrm{I}_{I} \quad a_{i}^{t} \cdot a_{i^{\prime}}=\delta_{i^{\prime}} & (i=1, \ldots, I), \\
\mathbf{B}_{I}^{t} \mathbf{B}_{I}=\mathrm{I}_{I} \quad b_{i}^{t} \cdot b_{i^{\prime}}=\delta_{i i^{\prime}} & (i=1, \ldots, I),
\end{array}
$$

where $\mathbf{A}_{I}=\left[a_{i}\right](1 \leq i \leq I)$ is an $N \times I$ matrix ( $a$ is a unit vector with dimension $N$ ) and $\mathbf{B}_{I}=\left[b_{i}\right](1 \leq i \leq I)$ is a $J \times I$ matrix ( $b$ is a unit vector with dimension $J$ ). In addition, $\boldsymbol{a}$ and $\boldsymbol{b}$ are eigenvectors satisfying

$$
\begin{aligned}
\mathbf{U U}^{t} a_{i}=\lambda_{i}^{2} a_{i} & (i=1, \ldots, I), \\
\mathbf{U}^{t} \mathbf{U} b_{i}=\lambda_{i}^{2} b_{i} & (i=1, \ldots, I),
\end{aligned}
$$

respectively, and so $\lambda_{i}^{2}$ are non-zero eigenvalues, which have to be distinguished from the mode eigenvalues $\kappa_{n}$.

If the receivers are placed in the water column and the water density is equal to one all around the receiver positions, which may be assumed without great risk in shallow water, then $\mathbf{R}$ in Eq.(3-63) becomes an identity matrix. Employment of this $\mathbf{R}$ in Eq.(362) leads to $\mathbf{V}=\mathbf{U}$. Hence, by using Eq.(3-67) with this relation, we have

$$
\mathbf{U}^{t} \mathbf{U}=\mathbf{B}_{I} \Lambda_{I}^{2} \mathbf{B}_{I}^{t},
$$

or using the vector $\boldsymbol{b}$ :

$$
\mathbf{U}^{t} \mathbf{U}=\sum_{i=1}^{I} \lambda_{i}^{2} b_{i} b_{i}^{t} .
$$


Here, since $\lambda_{i}$ are not zero, the inverse matrix $\Lambda_{I}^{-1}$ exists. Thus it follows from Eq.(3-73) that [43]

$$
\left(\mathbf{U}^{t} \mathbf{U}\right)^{-1}=\mathbf{B}_{I} \Lambda_{I}^{-2} \mathbf{B}_{I}^{t},
$$

or using vector the $b$ and $\Lambda_{I i k}^{-2}=\delta_{i k} / \lambda_{i}^{2}$ :

$$
\left(\mathbf{U}^{t} \mathbf{U}\right)^{-1}=\sum_{i=1}^{I} \frac{1}{\lambda_{i}^{2}} b_{i} b_{i}^{t}
$$

Substitution of $\mathbf{q}_{t}$ in Eq.(3-64) into Eq.(3-58) with the use of Eq.(3-67) and Eq.(3-75) yields the following identity

$$
\mathbf{p}_{I}=\mathbf{A}_{I} \mathbf{A}_{I}^{t} \mathbf{p},
$$

where $\mathbf{p}$ is the observed field data and $\mathbf{p}_{I}$ is the predicted field data obtained by using the solution $\mathbf{q}_{I}$. In general, $\mathbf{A}_{I} \mathbf{A}_{I}^{t}$ is not equivalent to an identity matrix and represents the resolution of $\mathbf{p}_{I}$. As the rank of the resolution matrix $\mathbf{A}_{I} \mathbf{A}_{I}^{\prime}$, i.e., I decreases, we have lower resolution of $\mathbf{p}_{\boldsymbol{I}}$.

Theoretically Eq.(3-75) and Eq.(3-76) can hold for any $\lambda_{i}(>0)$, but numerically becomes unstable if there exists $\lambda_{i_{0}} \approx 0$. If we use the EDM, these eigenvalues are excluded from Eq.(3-76), yielding

$$
\mathbf{q}_{I}=\left(\sum_{i_{1} \neq i_{0}}^{I} \frac{1}{\lambda_{i_{1}}^{2}} b_{i_{1}} b_{i_{1}}^{t}\right) \mathbf{U}^{t} \mathbf{p} \quad(\check{I} \leq I),
$$

if the following condition is met: 


$$
\frac{\boldsymbol{b}_{i_{0}}^{t} \cdot\left(\mathbf{U}^{t} \mathbf{p}\right)}{\lambda_{i_{0}}}<<\frac{\boldsymbol{b}_{i_{1}}^{t} \cdot\left(\mathbf{U}^{t} \mathbf{p}\right)}{\lambda_{i_{1}}} \quad\left(i_{1}=1, \ldots, \breve{I}\right)
$$

where $\lambda_{i_{1}}$ is a typical eigenvalue after excluding $\lambda_{i_{0}}$.

On the other hand, in the SLMS method [48], one may add a small value $\varepsilon$ in the diagonal term of $\mathbf{U}^{t} \mathbf{U}$, yielding

$$
\mathbf{q}=\left(\mathbf{U}^{t} \mathbf{U}+\varepsilon \mathbf{I}_{l}\right)^{-1} \mathbf{U}^{t} \mathbf{p}
$$

By adding $\varepsilon b_{i}$ to both sides of Eq.(3-72), it is seen that the set of eigenvectors $b$ also satisfy the following eigenvalue system

$$
\left(\mathbf{U}^{t} \mathbf{U}+\varepsilon \mathbf{I}_{I}\right) \boldsymbol{b}_{i}=\left(\lambda_{i}^{2}+\varepsilon\right) \boldsymbol{b}_{i} \quad(i=1, \ldots, I)
$$

with different eigenvalues. Based on eigenvector analysis, the inverse matrix of $\mathbf{U}^{t} \mathbf{U}+\varepsilon \mathbf{I}_{l}$, results in

$$
\left(\mathbf{U}^{t} \mathbf{U}+\varepsilon \mathbf{I}_{I}\right)^{-1}=\mathbf{B}_{I} \mathbf{E}_{l} \mathbf{B}_{I}^{t}
$$

with

$$
\mathbf{E}_{l i^{\prime}}=\left(\frac{\delta_{i i^{\prime}}}{\lambda_{i}^{2}+\frac{\varepsilon}{\lambda_{i}^{2}}}\right) \quad(i=1, \ldots, I)
$$

When $\lambda_{i} \gg>\varepsilon$, we may drop $\varepsilon / \lambda_{i}^{2}$ out of the denominator in Eq.(3-83) and so obtain the same diagonal terms as those in Eq.(3-76). On the other hand, when $\lambda_{i}<<\varepsilon$, the 
diagonal terms become close to $\lambda_{i}{ }^{2} / \varepsilon$ and so becomes negligible. As a result, this added small $\varepsilon$ term has the same effect as that in the eigenvalue decomposition approach.

In this way, by arranging the small eigenvalues in the singular matrix with the use of the EDM or the SLMS, we can obtain a stable output from the mode filter. The effectiveness of this method in mode filtering will be shown numerically in Section 3.4.

The generalized inverse used in the above methods leads to minimizing the vector norm $|\mathbf{p}-\mathbf{U q}|^{2}$ or $|\mathbf{p}-\mathbf{U q}|^{2}+\chi Z(\mathbf{q})[Z(\mathbf{q})$ : measure of smoothness and $\chi$ : Lagrange multiplier]. If the measured pressure $\mathbf{p}$ consists of a set of signals with noise, which obeys the Gaussian distribution with the covariance $\Gamma$, then we need to minimize $(\mathbf{p}-\mathbf{U q})^{t} \Gamma^{-1}(\mathbf{p}-\mathbf{U q})$ in order to maximize the following probability density function for p [43]:

$$
\Pi(\mathbf{p})=\frac{\left|\Gamma^{-1}\right|^{\frac{1}{2}}}{(2 \pi)^{\frac{4}{2}}} e^{-\frac{1}{2}\left(\mathbf{p}-\mathbf{U}_{\mathbf{q}}\right)^{r} \Gamma^{-1}(\mathbf{p}-\mathrm{Uq})} .
$$

As detailed in Ref. 77, the so-called maximum-likelihood inverse can be executed by a generalized inverse in transformed coordinates.

In this section, to reduce the error caused by the interference with other modes when using the asymptotic Hankel transform with a sliding window, we exploited mode filtering by incorporating data obtained with a fixed vertical array of receivers. When this filtering process becomes unstable due to the effect of a singular matrix, we may utilize the EDM or the SLMS for inversion of the singular matrix in order to provide reliable mode separation. In the next section, the asymptotic Hankel transform with a sliding window will be applied together with mode filtering to the pressure field for a laterally inhomogeneous medium; the field will be synthesized numerically using adiabatic mode theory. 


\subsection{Analysis of simulated data}

In this section, we demonstrate through a simulated example that the methods discussed in the previous sections to detect the local eigenvalues are effective. These methods are applied here to the pressure field in a laterally inhomogeneous waveguide, which is numerically simulated by using the adiabatic mode theory explained in Chapter 2.2.

\subsubsection{Ocean and seabed environment}

The laterally inhomogeneous shallow water model used here is shown in Figures 3-9 to 3-14. It is based on a range-dependent, multi-layered sediment model, which we will detail in Sections 5.1 and 5.4. As assumed in the previous two sections, the environment is taken to be cylindrically symmetric around the $z$ axis, where either the source or receiver array will be placed.

The bottom consists of three sediment layers, whose properties at $r=0$ are indicated in Figure 3-9. The density and the attenuation coefficient take on different values in different layers but remain constant within each layer. Also, the sound speed varies continuously in the vertical direction in each layer, but becomes discontinuous across the layer interfaces. The sound speed profile in each layer is described by an $n^{2}$-linear curve:

$$
c(r, z)=c_{j}(r)\left[1-\frac{2 g_{j}(r)}{c_{j}(r)}\left[z-h_{j}(0)\right]\right]^{-\frac{1}{2}} \quad\left(h_{j}(r) \leq z \leq h_{j+1}(r)\right),
$$

where $h_{j}(r)$ is the depth of the layer interface, $c_{j}(r) \equiv c\left(r, h_{j}(0)\right)$ is the sound speed at $z=h_{j}(0)$, and $g_{j}(r)$ is the sound speed gradient in the jth layer. Note that the curve in Figure 3-9 shows the profile at $r=0$, but becomes different at other ranges due to its range dependence. Here the first two sediment layers are subject to lateral variation, but the lowest layer (subbottom) is taken to be range-independent. 


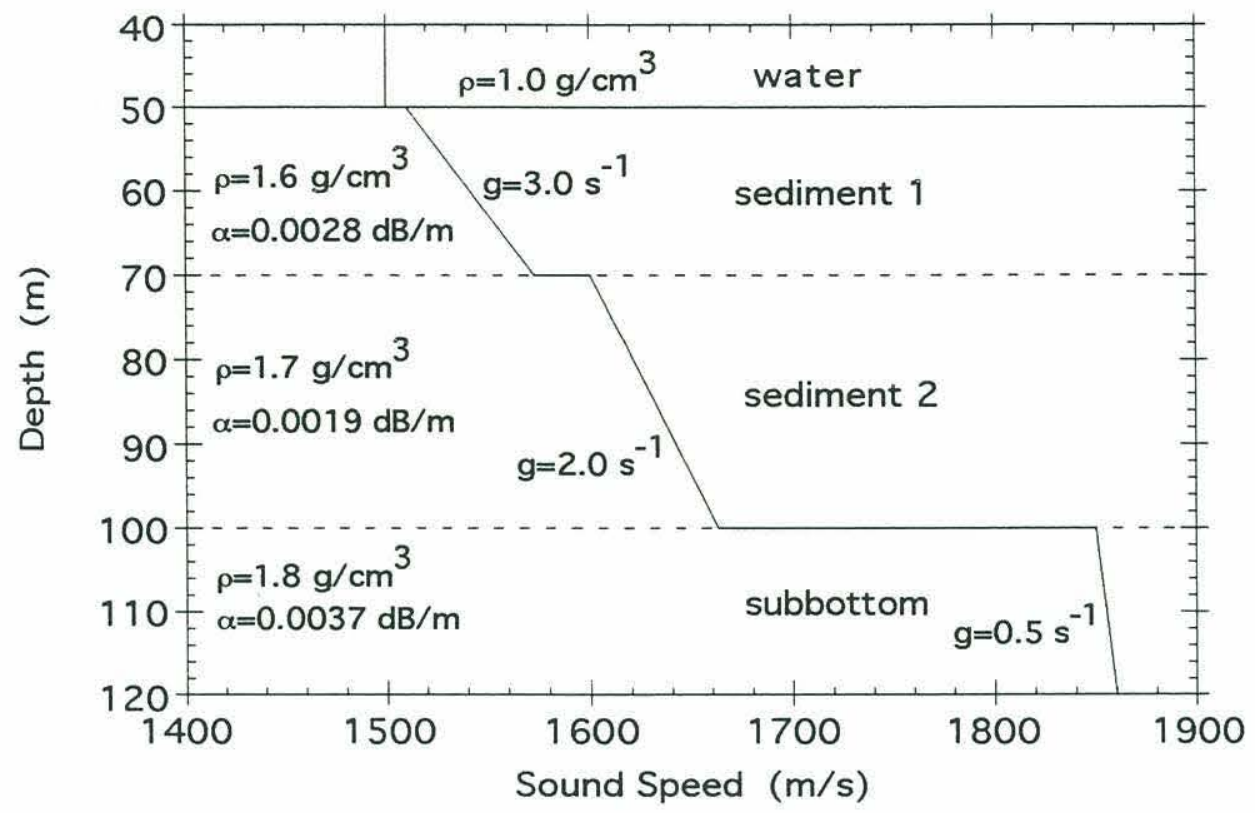

Figure 3-9: Sediment sound speed profile at $r=0$ and other geoacoustic parameters in the shallow water bottom model.

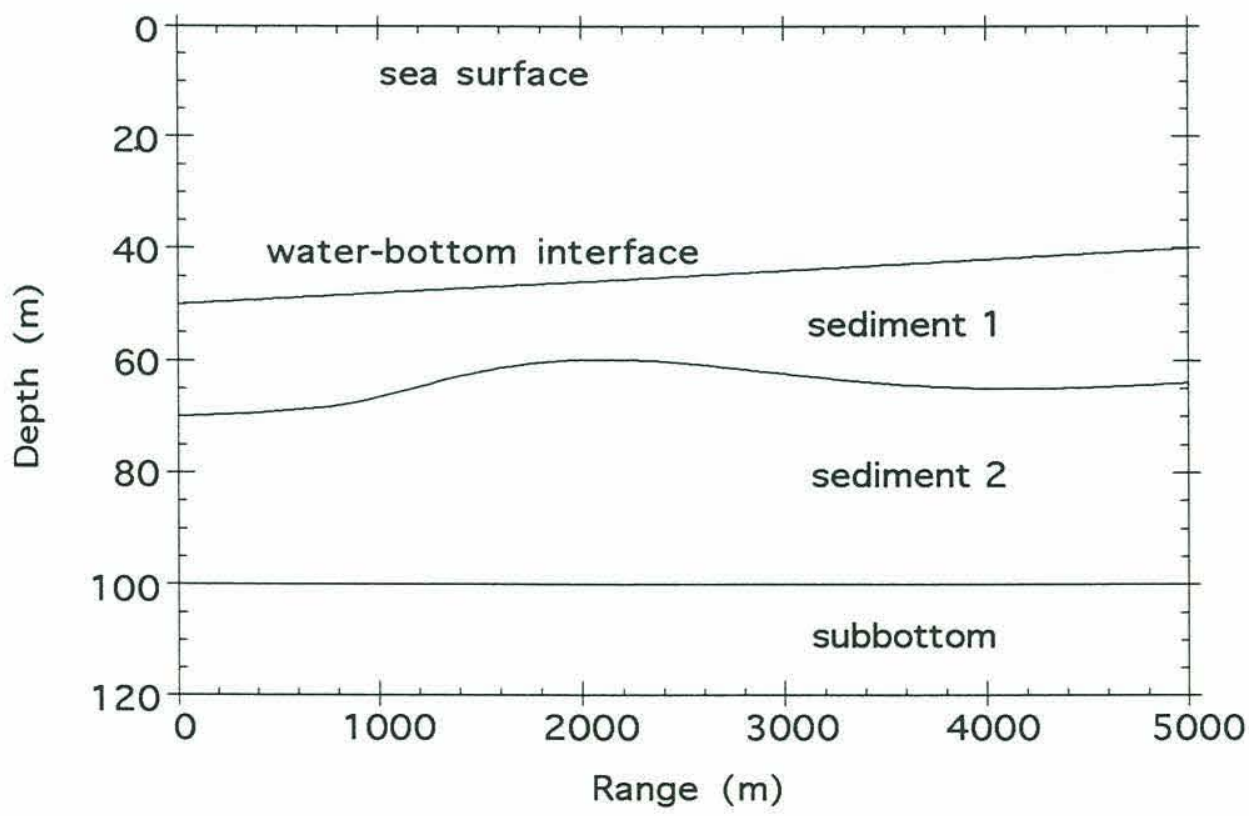

Figure 3-10: Lateral variation of the layer interfaces in the shallow water bottom model. 


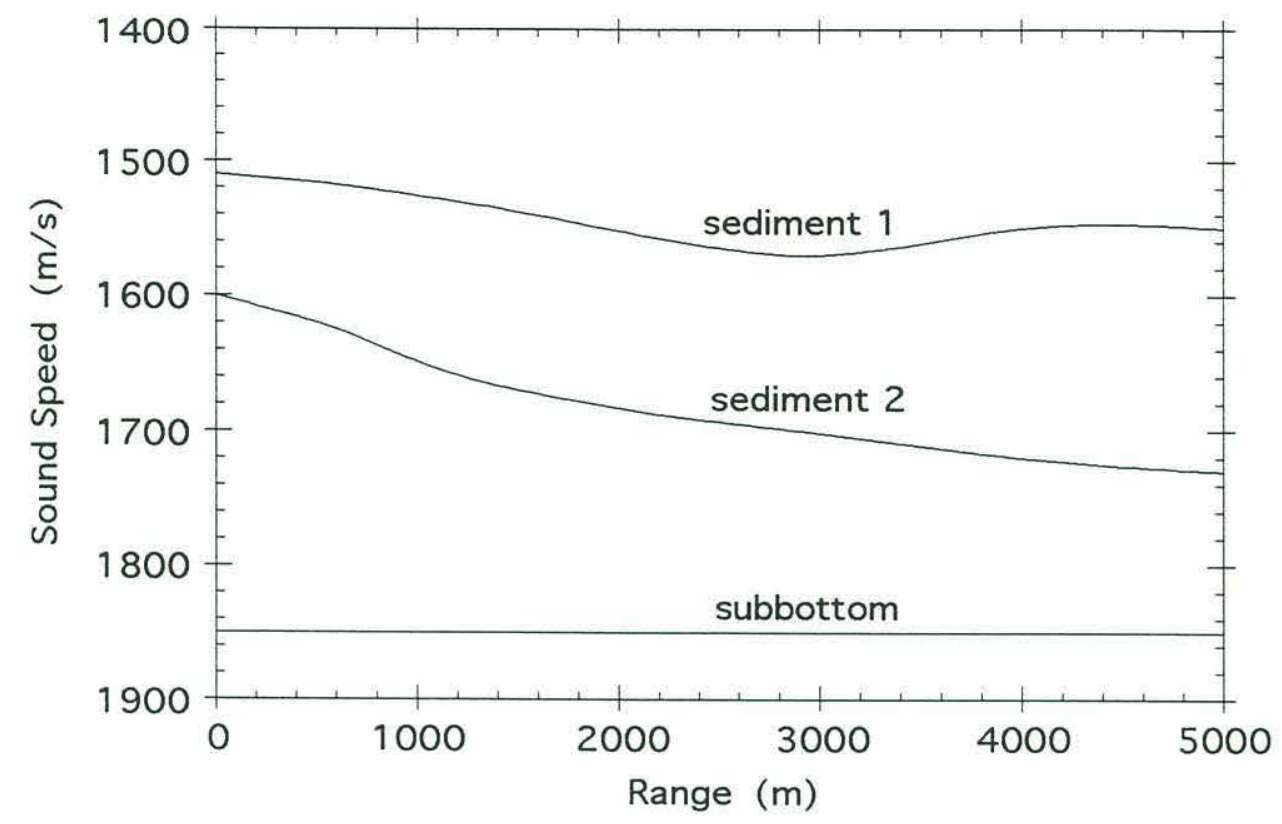

Figure 3-11: Lateral variation of the sound speed at each sediment interface in the shallow water bottom model.

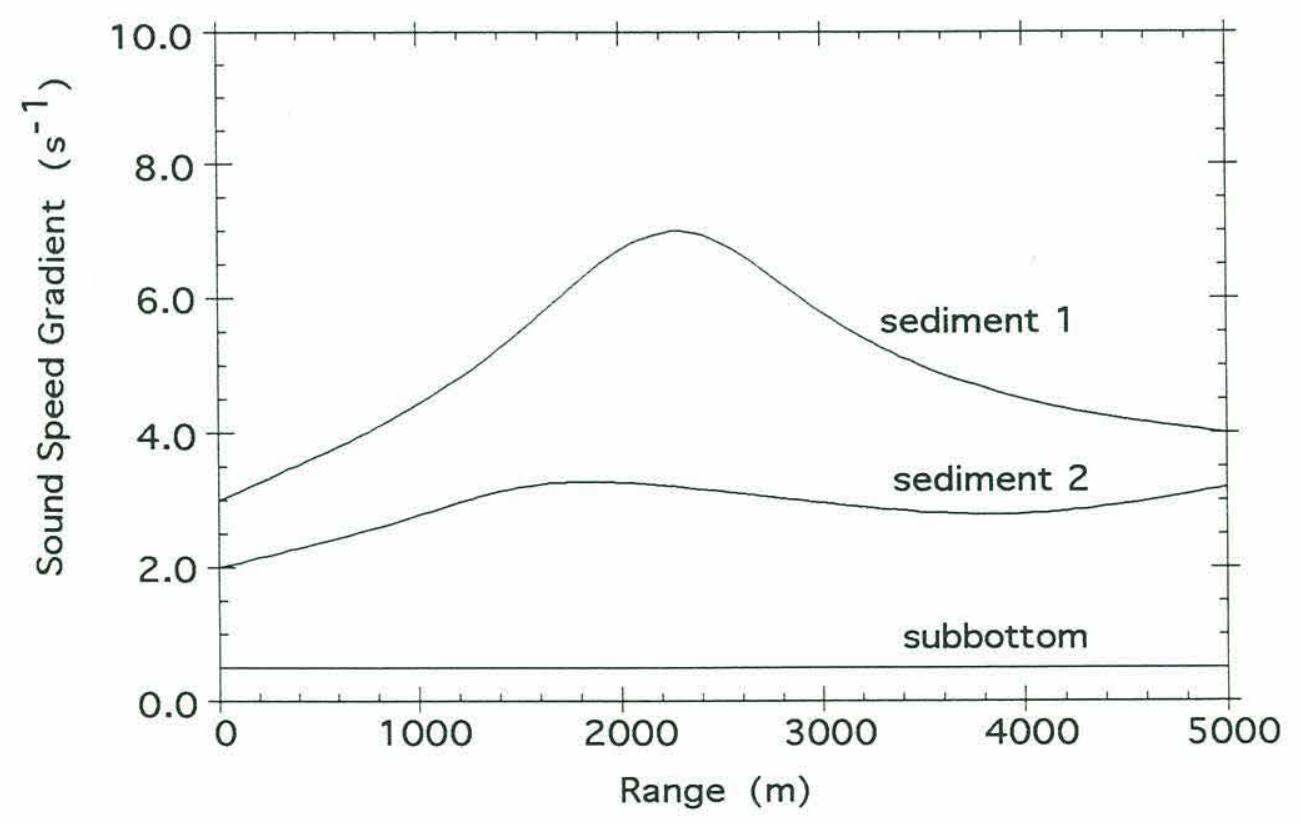

Figure 3-12: Lateral variation of the sound speed gradient in each sediment layer in the shallow water bottom model. 


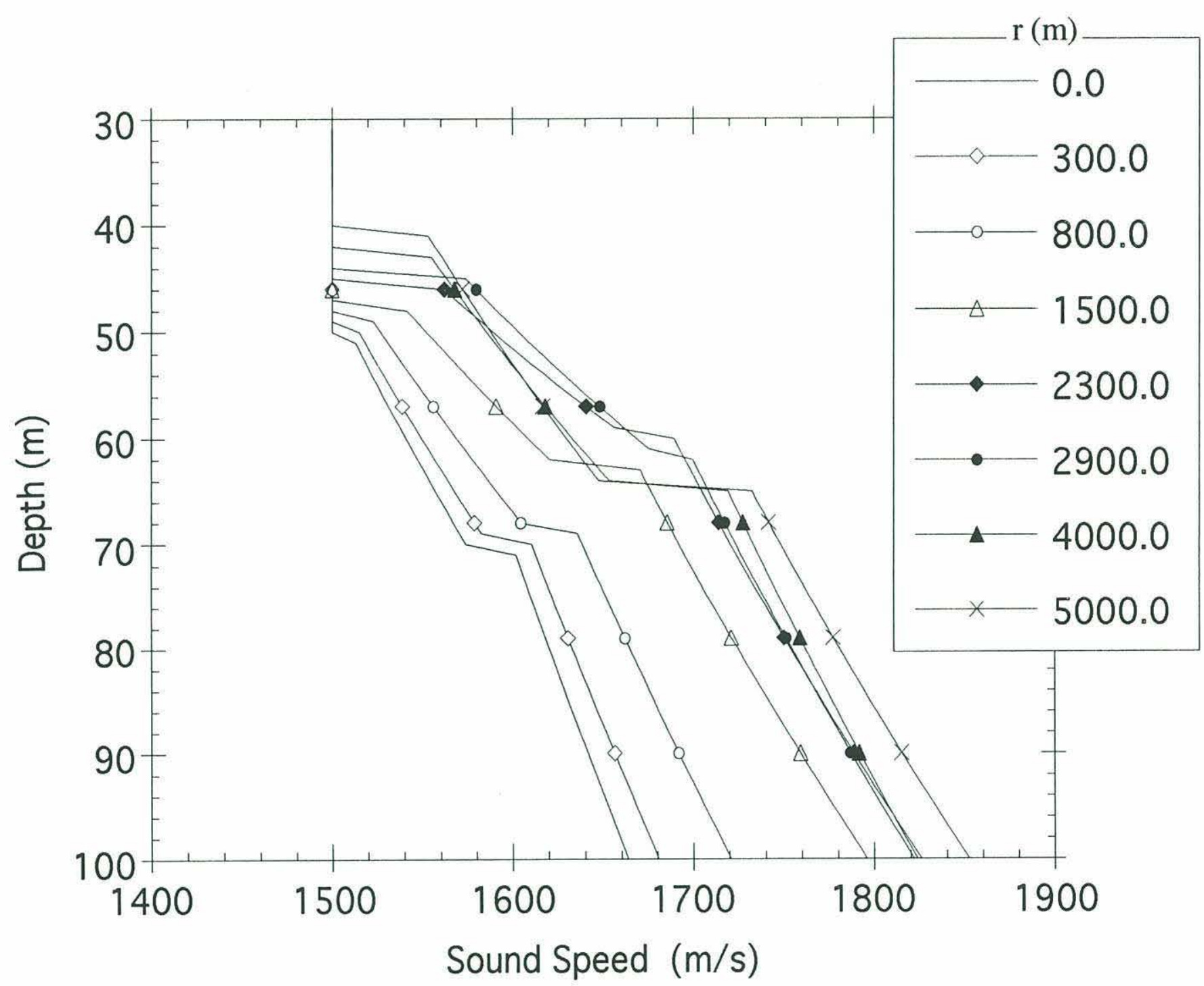

Figure 3-13: Sediment sound speed profiles at different ranges in the shallow water bottom model. 


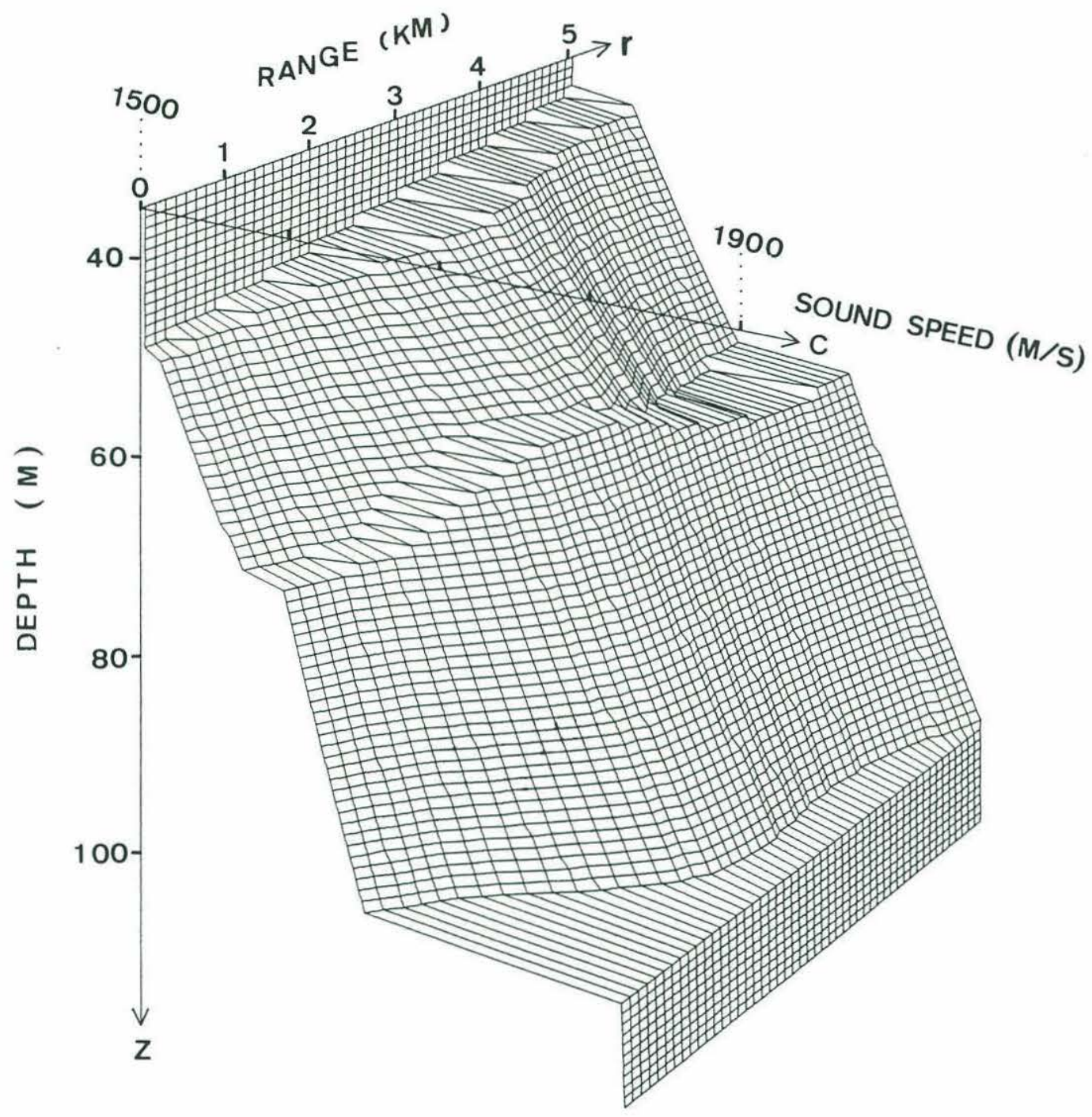

Figure 3-14: Three-dimensional picture of the sediment sound speed profiles in the shallow water bottom model. 
Figure 3-10 shows the variation with range of the layer interface and the bathymetry. These interfaces, including the water-bottom interface, are set so that they vary smoothly with range to meet the adiabatic condition. The sound speed in the sediment also has lateral variation, which is provided through range-dependent coefficients $c_{j}(r)$ and $g_{j}(r)$ in the $n^{2}$-linear curve. Figures 3-11 and 3-12 show the lateral variation of the sound speed at the interface depths and the sound speed gradient in each layer, respectively. The geoacoustic parameters in the subbottom are constant due to its range independence. The sound speed profiles in the sediment at various ranges are shown in Figure 3-13, while a 3-D picture of the profiles is exhibited in Figure 3-14. For simplicity, the sound speed in the water column is taken to be constant such that $\mathrm{c}=1500 \mathrm{~m} / \mathrm{s}$, and the water density is fixed to be $1 \mathrm{~g} / \mathrm{cm}^{3}$. Thus, we can simulate the pressure field by employing adiabatic mode theory in this laterally inhomogeneous waveguide.

\subsubsection{Acoustic environment}

Here, we will consider a point source with a frequency of $75 \mathrm{~Hz}$; the water depth at $r=0$ corresponds to $2.5 \lambda$, where the wavelength $\lambda=20 \mathrm{~m}$ in the water column. Figure 3-15 shows the first nine modes at this range $(r=0)$. The local eigenfunctions $\left|u_{n}(z, 0)\right|$ are plotted versus depth $z$ and are individually normalized so that their maximum absolute values are unity. Also, the local eigenvalues of these modes are indicated in the complex wavenumber domain in Figure 3-16. As seen from this figure, the real parts of the eigenvalues of the first five modes are greater than the wavenumber at the depth of the subbottom surface $k_{4}$ and less than $k_{1}=2 \pi / \lambda$, so that these modes are trapped between the sea surface and the subbottom interface. The modes with eigenvalues that are smaller than this wavenumber are similar to leaky modes because of the small sound speed gradient in the subbottom layer. As this gradient goes to zero, the distance of these adjacent modes in the $k_{r}$-plane decreases and eventually they become part of the modal continuum when $g_{4}=0$. 


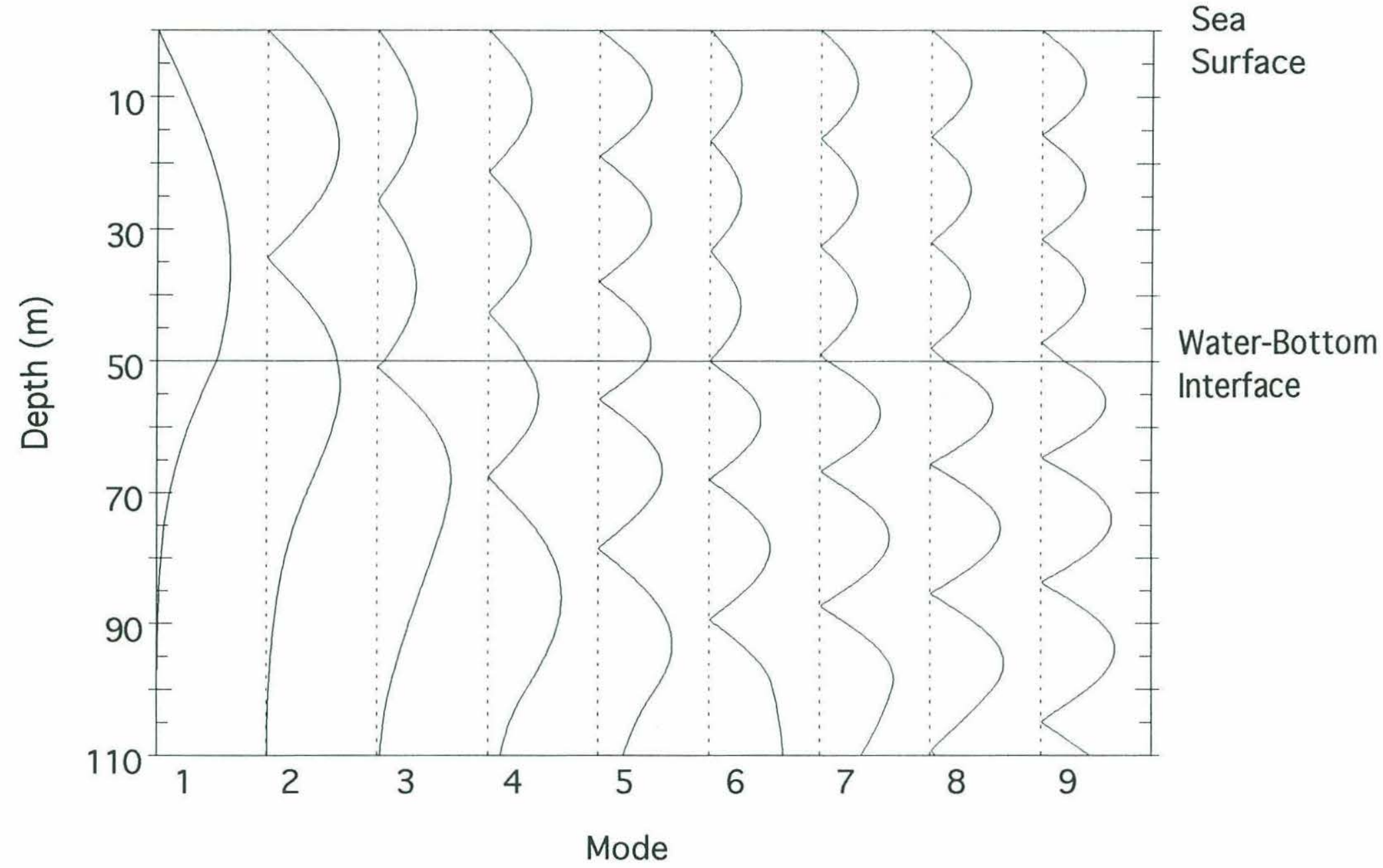

Figure 3-15: Local eigenfunctions $\left|u_{n}(z, 0)\right|$ of the first nine modes excited at $r=0$ in the shallow water model shown in Figures 3-9 to 3-14. 


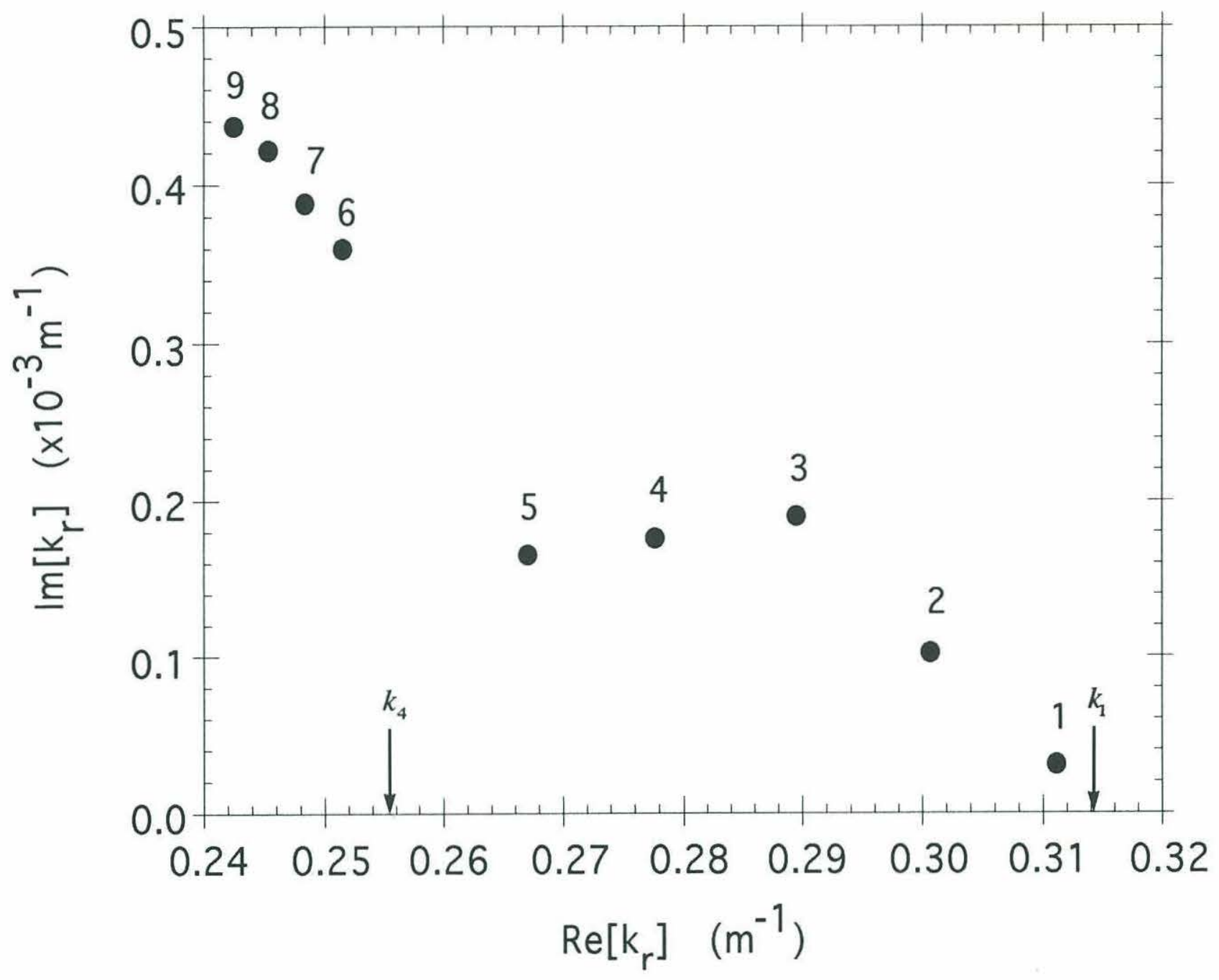

Figure 3-16: Local eigenvalues of the first nine modes represented in the complex $k_{r}$-plane. 


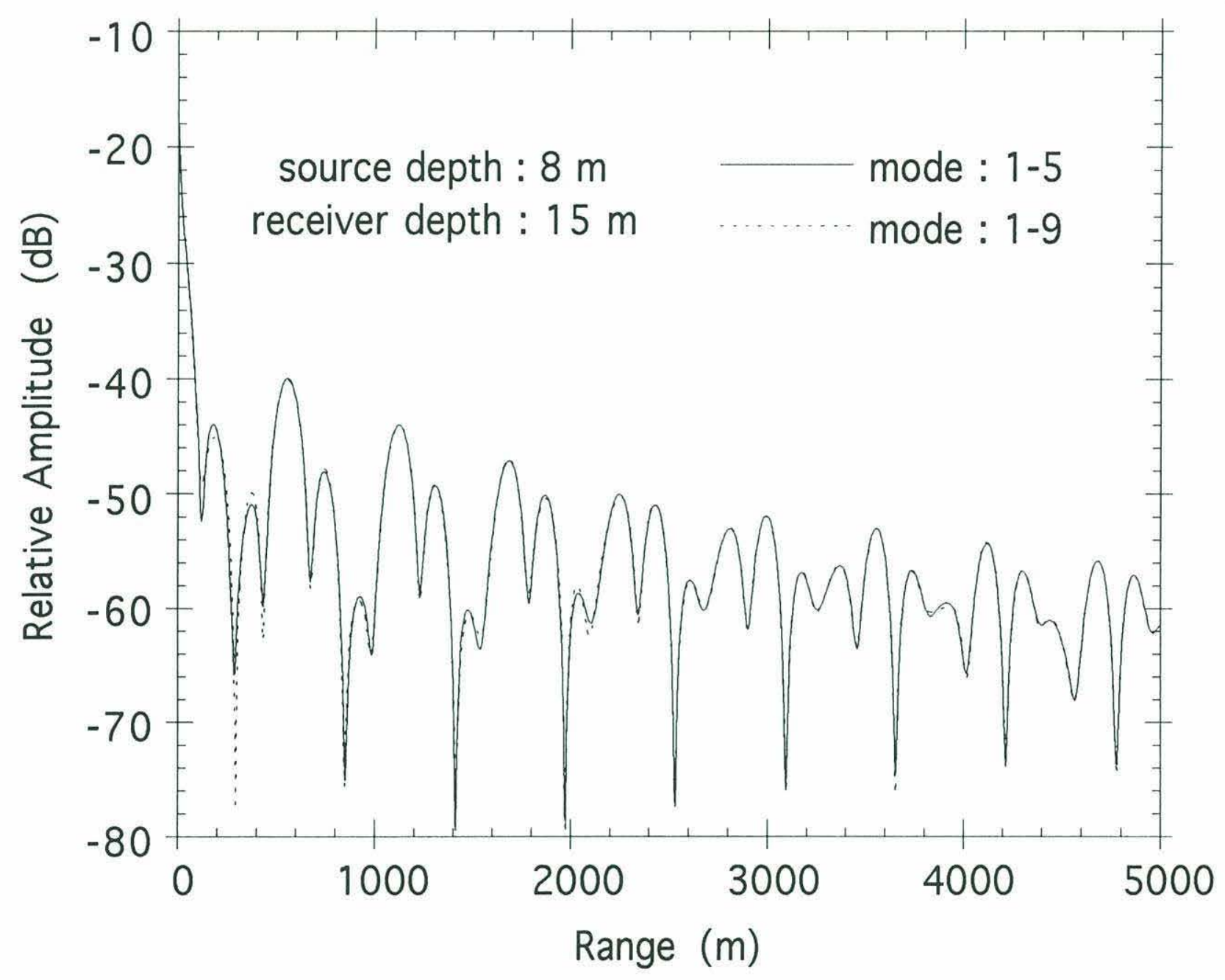

Figure 3-17: Comparison of the pressure field consisting of the first five modes and the pressure field consisting of the first nine modes. The pressure field is simulated by using normal mode theory in a horizontally stratified waveguide which has the bottom properties shown in Figure 3-9. 
In order to examine the effect of these higher modes, let us temporarily consider a horizontally stratified environment having the same properties as that at $r=0$. The pressure field $p\left(r, z, z_{0}\right)$ is then represented by the sum of those modes based on Eq.(2-6) in the normal mode theory. Thus we can compare two kinds of fields: One is a sum of the lowest five modes and the other is a sum of the lowest nine modes which includes the higher modes. These two results are compared in Figure 3-17, where we have plotted the relative amplitude, $20 \log _{10}\left|p\left(r, z, z_{0}\right)\right|$, due to a point source of unit amplitude at $r=1 \mathrm{~m}$. As seen from a comparison of these results, we cannot recognize substantial differences in the relative amplitudes. Thus we will basically use the lowest five modes to simulate the pressure fields. When the effect of the higher modes, however, is examined, these higher modes will be used in simulating the fields.

Now in the case of the range-dependent environment specified above, the local modes vary with range so as to satisfy the depth equation in Eq.(2-8). Since the local eigenvalues $\kappa_{n}(r)$ must be used as a measure for evaluating the results, which will be obtained from the output spectrum of the Hankel transform with the sliding window, we need to have highly accurate values of $\kappa_{n}(r)$ for comparison. We obtained these eigenvalues every $5 \mathrm{~m}$ in range by solving the range-dependent characteristic equation. In this process, we first obtained the approximate values for the local eigenvalues by utilizing Eq.(5-23), which will be derived for the purpose of relating the perturbed local eigenvalues and the perturbed geoacoustic parameters in an inverse problem in Chapter 5; these approximate values can then be converged to highly accurate values by using the Newton-Raphson method iteratively $[54,78]$. The local eigenvalues thus found for modes one through nine are shown as a function of range in Figure 3-18.

As seen from this figure, the range variation of the local eigenvalues of modes three through five is relatively large, because these three modes are strongly affected by the top two range-dependent sediment layers. This fact can also be inferred from the mode shapes in Figure 3-15. Of particular interest here is that the range variation of the local 


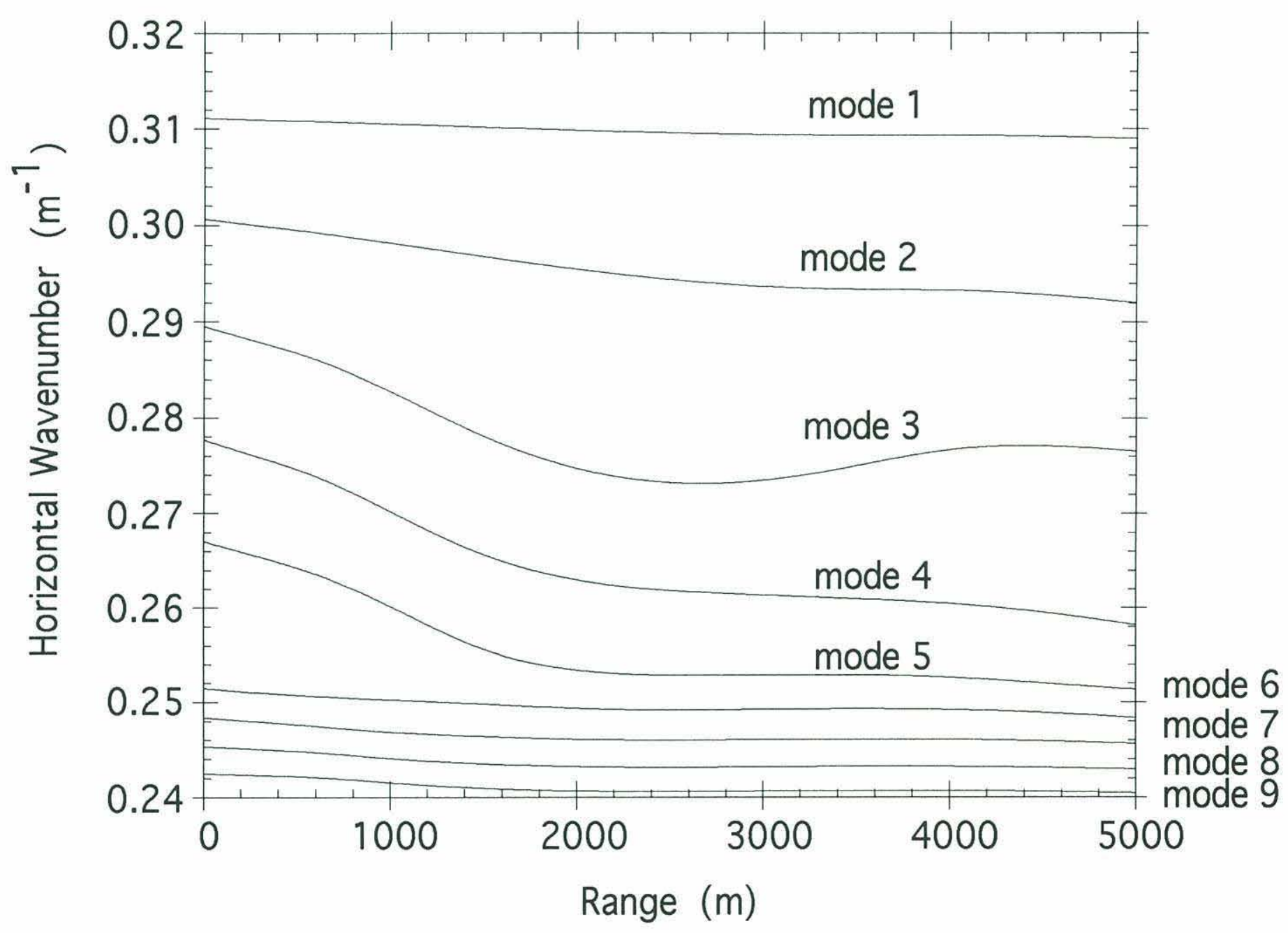

Figure 3-18: Local eigenvalues of the first nine modes as a function of range; the modes are obtained by using adiabatic mode theory in the model of a laterally inhomogeneous shallow water waveguide shown in Figures 3-9 to 3-14. 


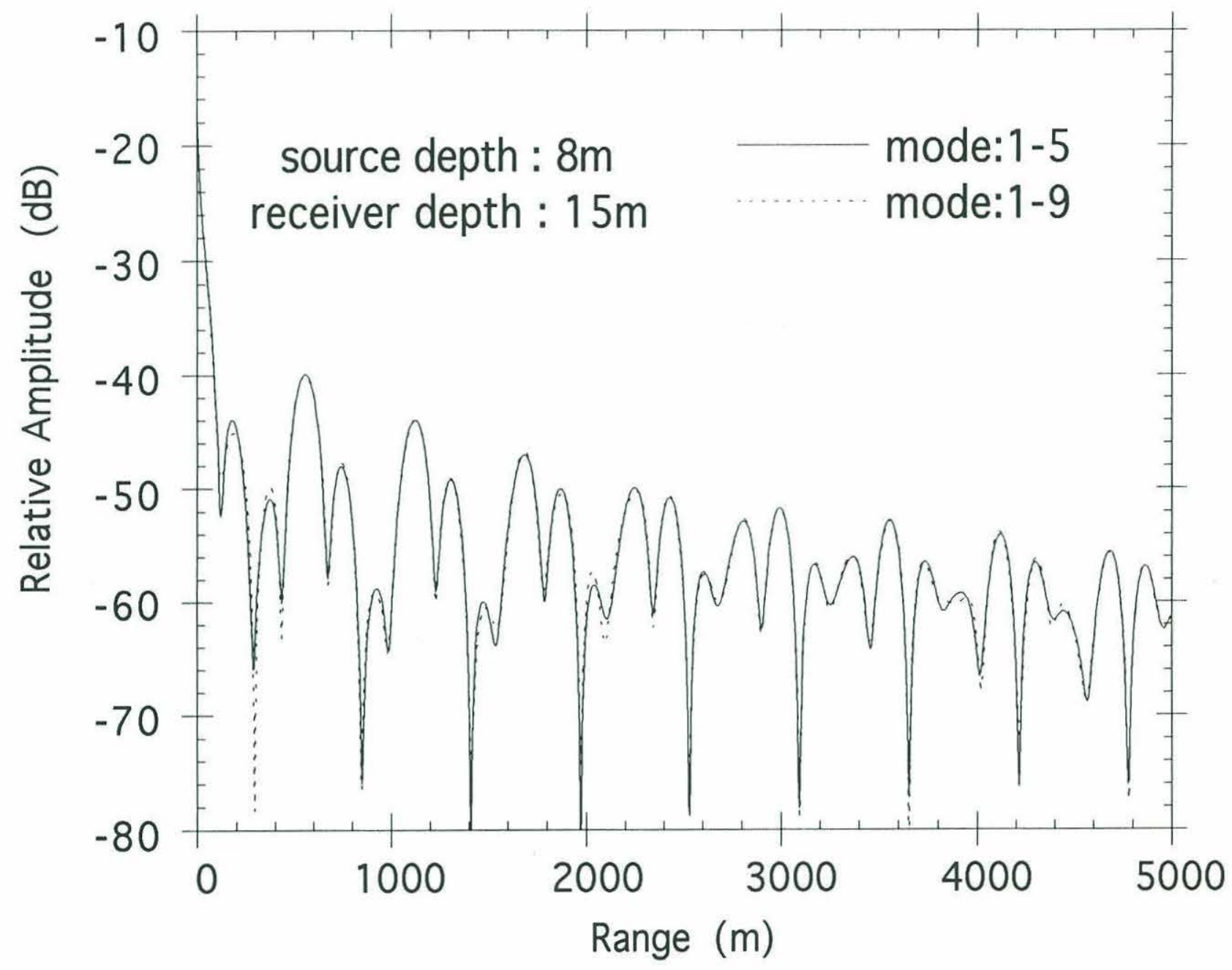

Figure 3-19: Comparison of the pressure field consisting of the first five modes and the pressure field consisting of the first nine modes. The pressure field is simulated by using adiabatic mode theory in the model of a laterally inhomogeneous shallow water waveguide shown in Figures 3-9 to 3-14. 
eigenvalue of the third mode shows a different behavior. Namely, as the water depth decreases with range, the local eigenvalues of the other modes decrease monotonically with range, whereas the local eigenvalue of the third mode first decreases, and then at a range of about $2500 \mathrm{~m}$, begins to increase. Apparently the third mode is affected more intensely by the variation in sediment properties than by bathymetric change.

Let us check the effect of the higher modes in this range-dependent environment. Figure 3-19 compares the pressure fields with and without those higher modes (modes 6-9) in the adiabatic mode sum in Eq.(2-12). Again we cannot recognize substantial differences between the two results.

In this way, we can make use of the pressure field that is synthesized by a sum of adiabatic modes. Once again, the objective in this chapter is to recover the rangedependent local eigenvalues from the given pressure field.

\subsubsection{Application of an asymptotic Hankel transform with a sliding window}

First let us apply a conventional asymptotic Hankel transform to the simulated pressure fields. In this transform, the rectangular window is used to truncate the field at 5 $\mathrm{km}$, so that all range-dependent components are weighted equally. For the horizontally stratified case in Figure 3-17, the output spectrum shown in Figure 3-20 clearly indicates that each peak corresponds to an individual mode. Note that several small peaks around the dominant ones are sidelobes caused by using the rectangular window in the transform. In contrast, as seen from Figure 3-21 for the range-dependent case in Figure 3-19, the peaks in the output spectrum are not distinctive except for the first mode. The peak of the second mode is split and the third mode peak is very hard to distinguish. As expected from the variation of $\kappa_{n}(r)$ in Figure 3-18, the latter result for the third mode is due to the interference with the adjacent fourth mode, which actually shares the same wavenumber at a different range. 


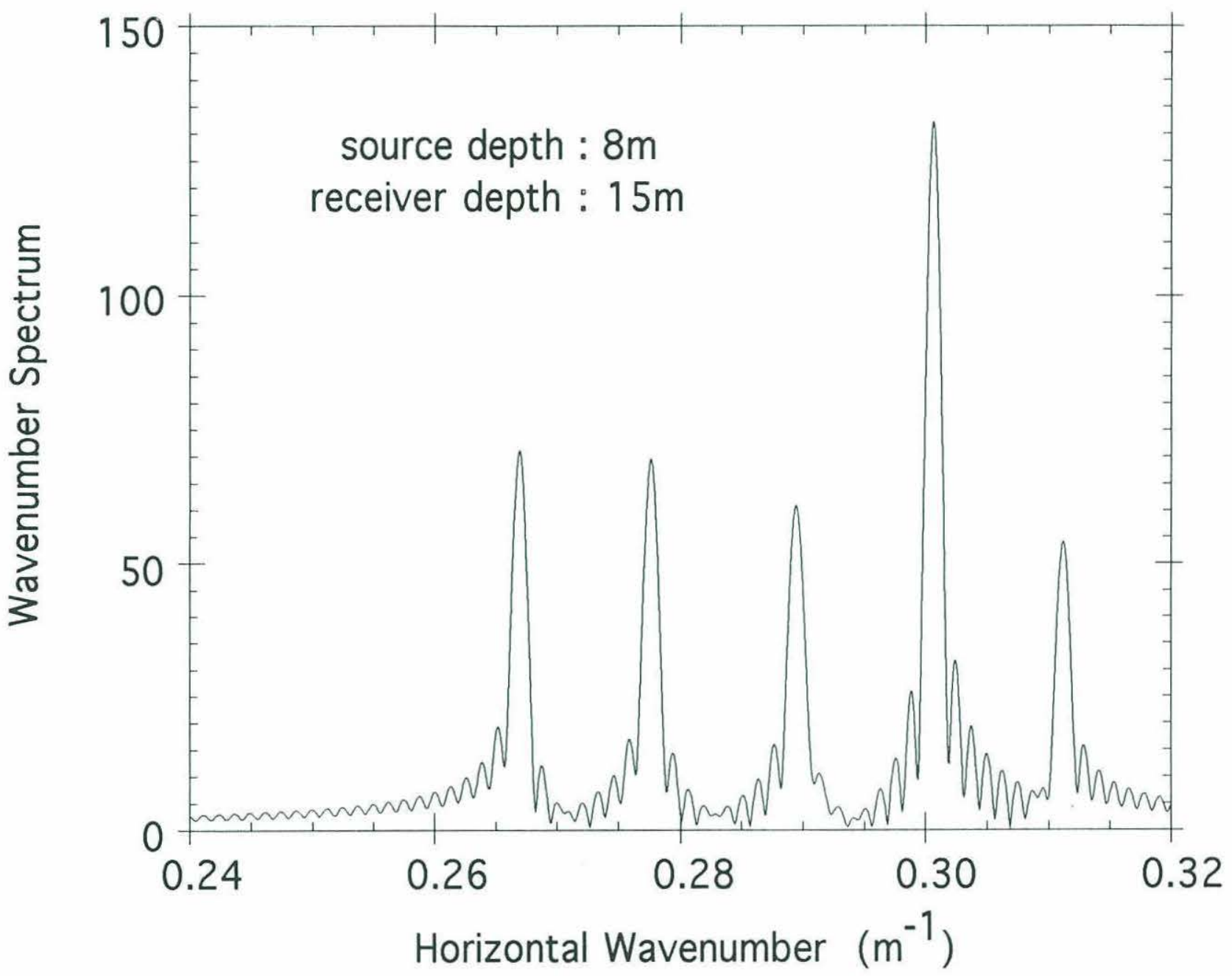

Figure 3-20: Wavenumber spectrum obtained by applying the asymptotic Hankel transform to the pressure field over a finite range $(5 \mathrm{~km})$ in the horizontally stratified waveguide which has the bottom sediment shown in Figure 3-9. The pressure field is simulated by using normal mode theory. 


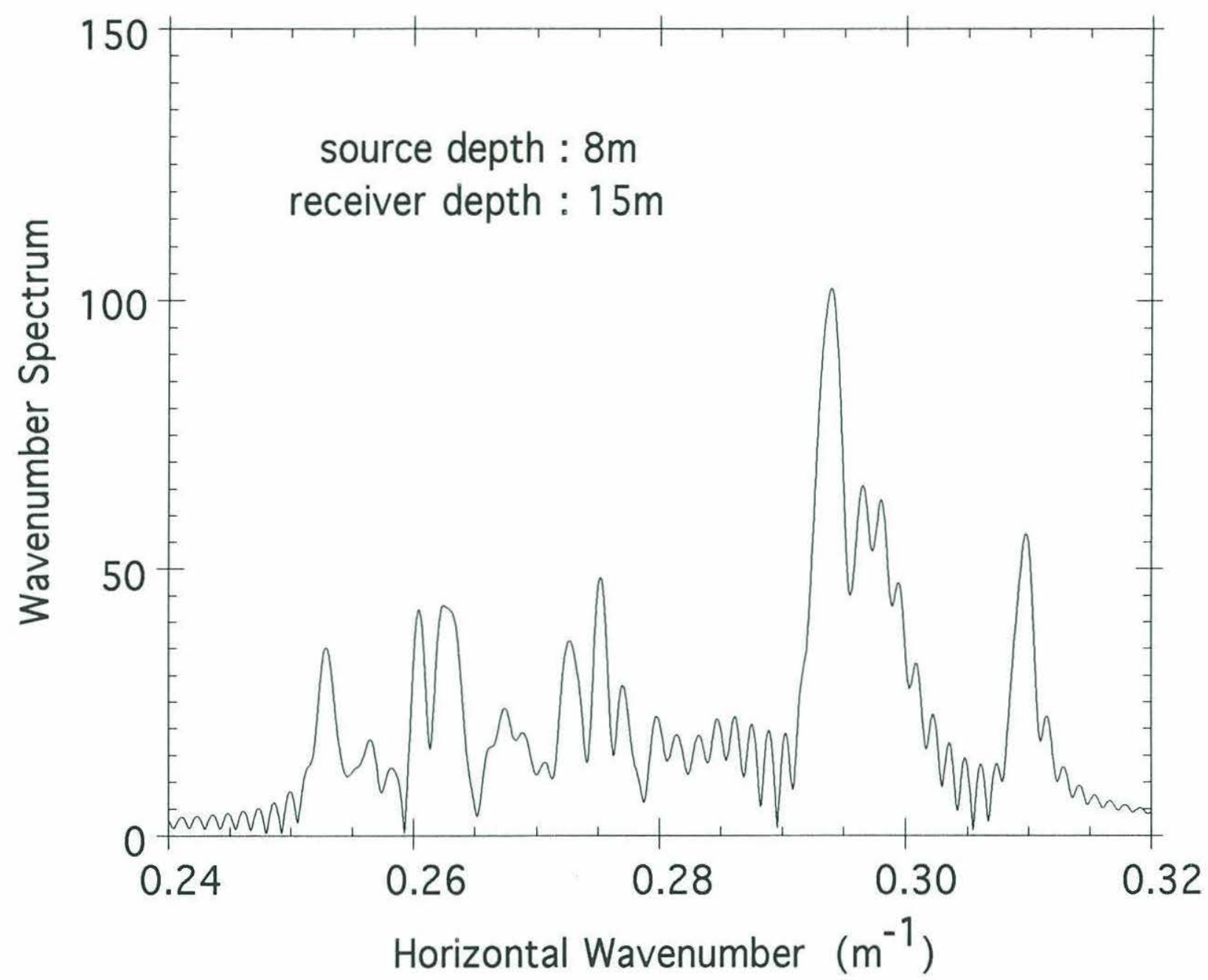

Figure 3-21: Wavenumber spectrum obtained by applying the asymptotic Hankel transform to the pressure field over a finite range $(5 \mathrm{~km})$ in the laterally inhomogeneous waveguide. The pressure field is simulated by using adiabatic mode theory and the shallow water model shown in Figures 3-9 to 3-14 is used as this waveguide. 
Next let us examine the local character of the above spectrum by using a sliding Hanning window. Figures 3-22(a) and (b) show the output spectrum of Eq.(3-30) when using a Hanning window with a length $(L)$ of $1000 \mathrm{~m}$ and $2000 \mathrm{~m}$, respectively. Here the center position of this window is slid every $50 \mathrm{~m}$. In the case of $L=1000 \mathrm{~m}$, the spectrum became unstable due to the interference of adjacent modes; this result stems from a short window length. The result for $L=2000 \mathrm{~m}$ improves this situation at the cost of losing the local character, but even in this case we cannot distinguish clearly the peak of mode 3 through mode 5 in the region around $r=1500 \mathrm{~m}$.

In order to improve this result, let us utilize a mode filter by incorporating data from a vertical receiving array at $r=0$. The array considered here consists of 10 receivers placed in the water column every $5 \mathrm{~m}$ as shown in Figure 3-23. On use of Eq.(3-64) the pressure field can be separated by modes and then the above asymptotic Hankel transform with the Hanning window may be applied to the output of this mode filter. Figure 3-24 shows a set of range-dependent spectra for each mode when using $L=1000 \mathrm{~m}$. Figures 3-25(a) and (b) show the peak positions of mode 3 and mode 5 in Figures 3-24, respectively, and these peak positions are compared with the local eigenvalues. We chose only to show the peak positions of modes 3 and 5 because they are representative of the peak positions for the other modes. It is observed that the departure from the exact values is noticeable at the range where the curvature of the local eigenvalue is large. To effect comparison, the difference of these two curves, i.e., the amount of the departure from the local eigenvalue, is plotted again in Figures 3-26. This difference is further amplified for the case of $L=2000 \mathrm{~m}$ as shown in Figures 3-27, which we discussed in Section 3.2. Namely, as the window length increases, the departure from the exact eigenvalue becomes larger.

In order to study the effect of the higher modes on the performance of the mode filtering operation, let us apply the same method to the pressure field that is synthesized by including those higher modes. In this case, some of the eigenvalues in Eq.(3-68) 


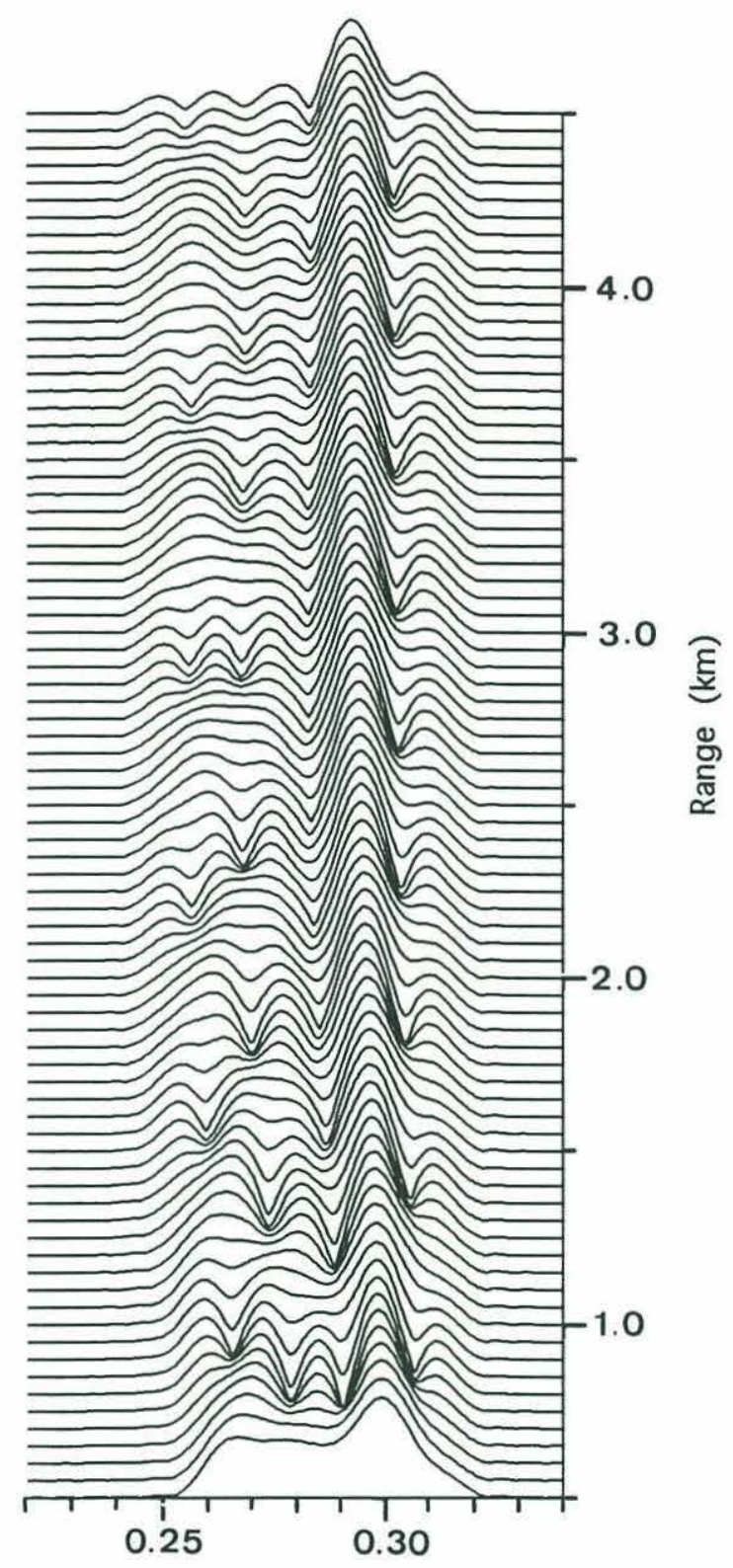

Horizontal Wavenumber $\left(\mathrm{m}^{-1}\right)$

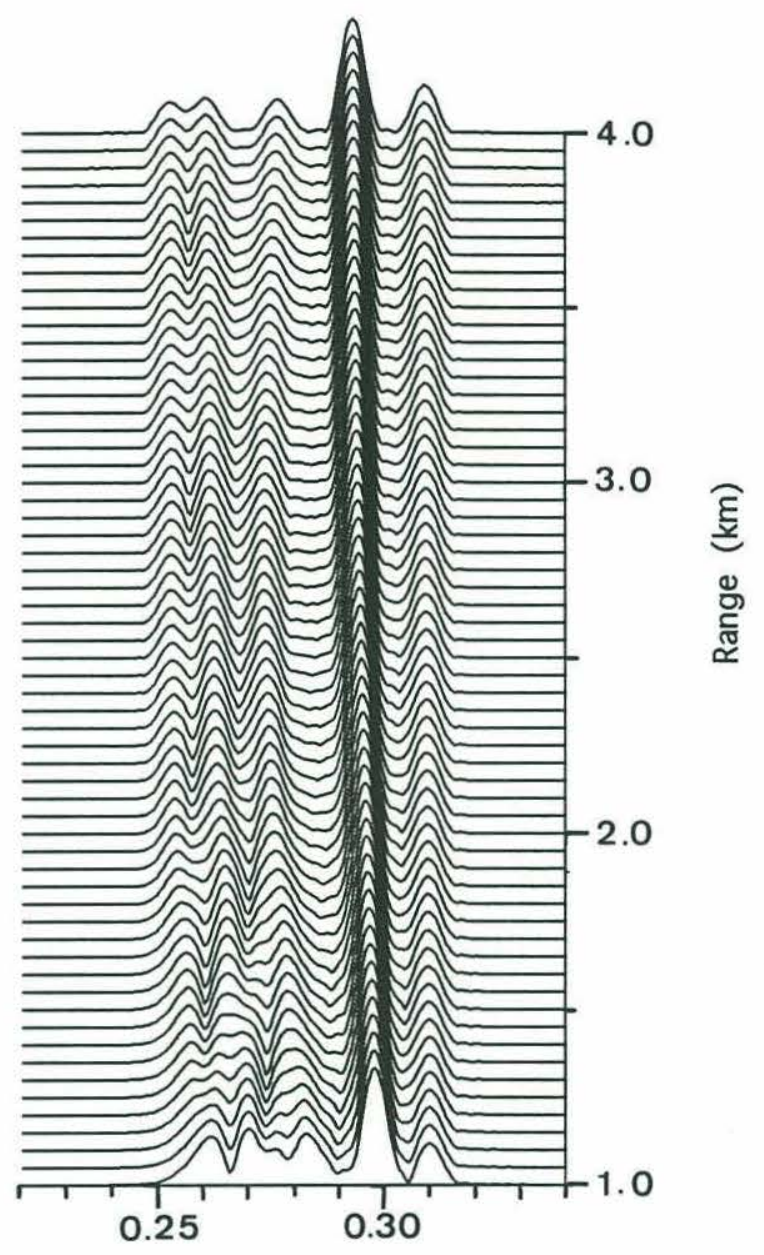

Horizontal Wavenumber $\left(\mathrm{m}^{-1}\right)$

(a)

(b)

Figure 3-22: Range-dependent evolution of the wavenumber spectrum obtained by applying the asymptotic Hankel transform with a sliding Hanning window to the pressure field which is simulated for the model of a laterally inhomogeneous shallow water waveguide shown in Figures 3-9 to 3-14. Source depth is $8 \mathrm{~m}$ and receiver depth is $15 \mathrm{~m}$ : (a) window length $=1000 \mathrm{~m}$ and (b) window length $=2000 \mathrm{~m}$. 


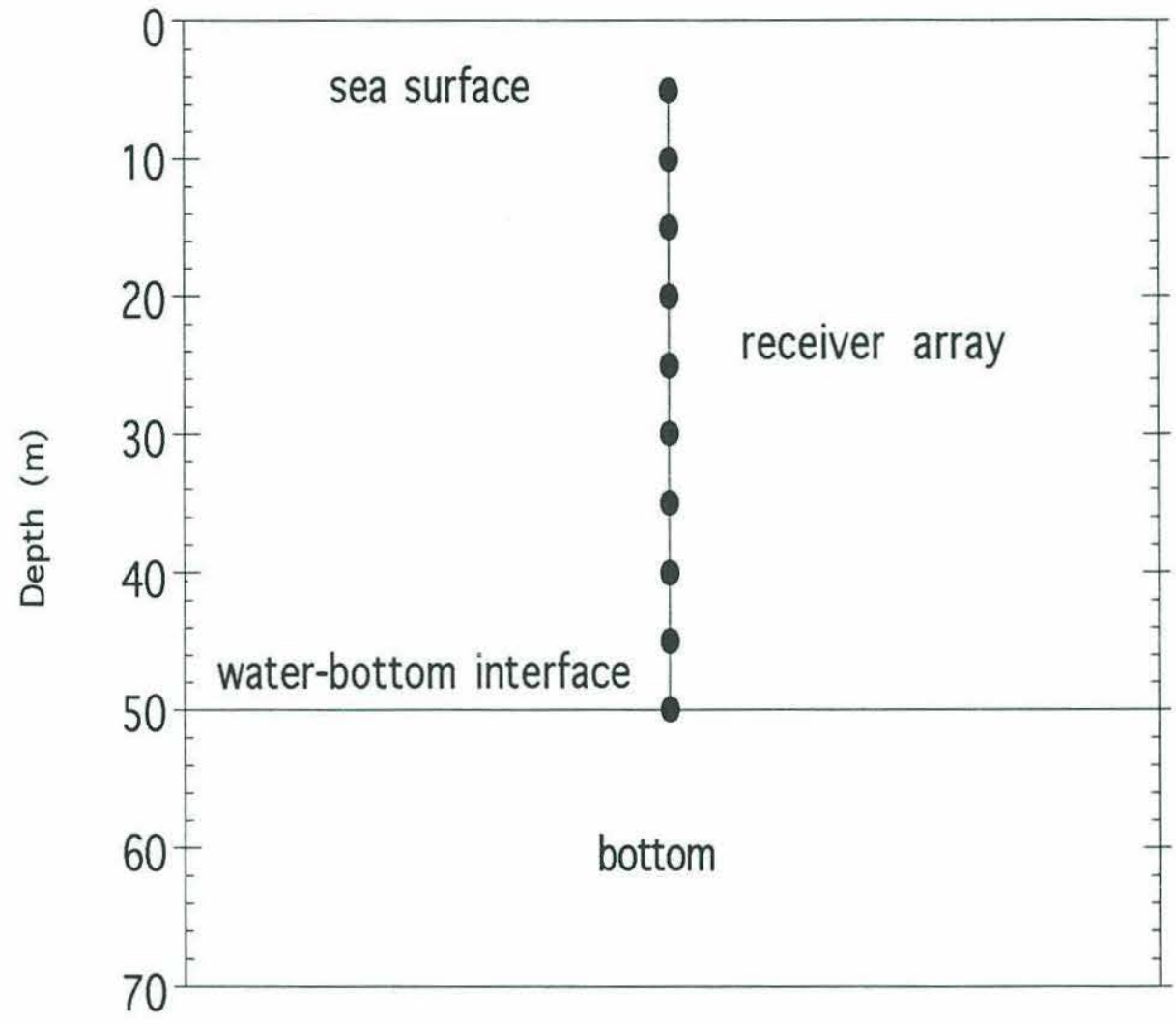

Figure 3-23: Geometry of a vertical array of receivers fixed at $r=0$ in the shallow water model. 


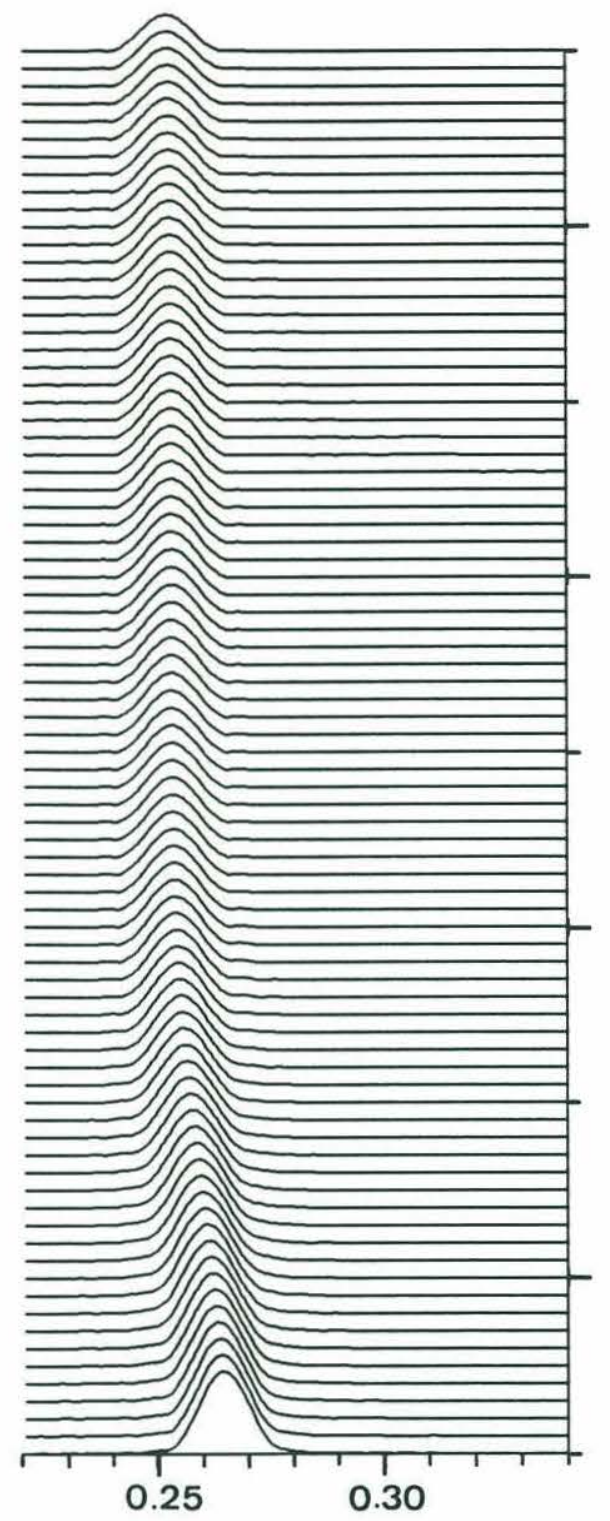

Horizontal Wavenumber $\left(\mathrm{m}^{-1}\right)$

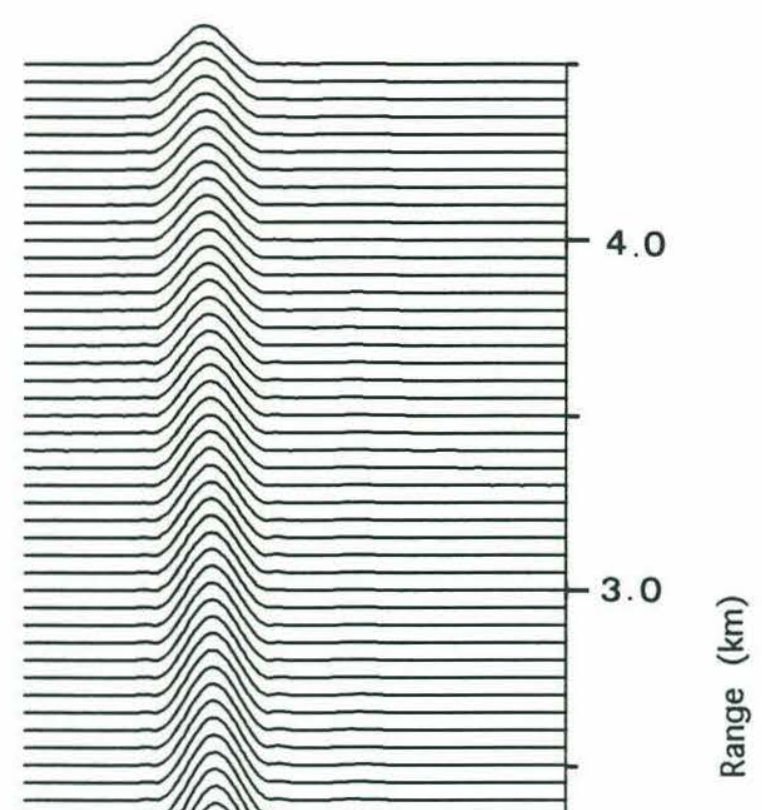

2.0

1.0

Mode 5

Mode 4

Figure 3-24: Range-dependent evolution of the individual modal spectra obtained by mode filtering with use of the vertical array in Figure 3-22. 


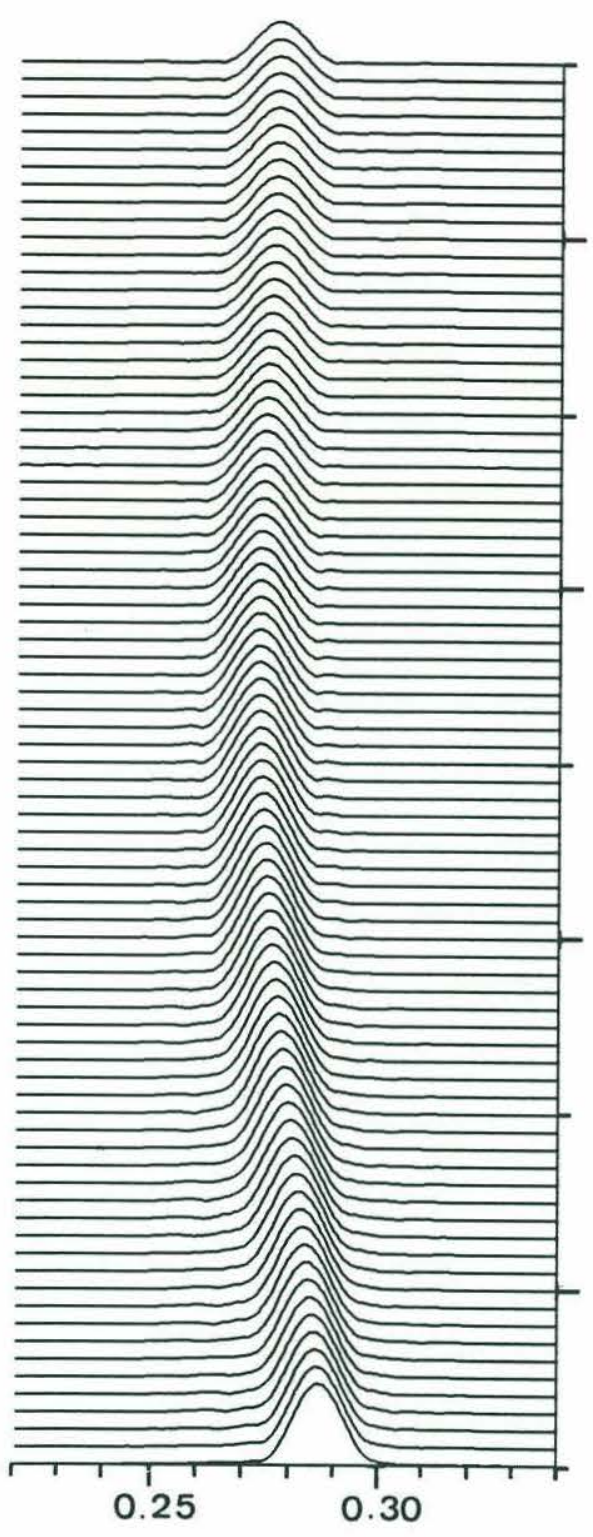

Horizontal Wavenumber $\left(\mathrm{m}^{-1}\right)$

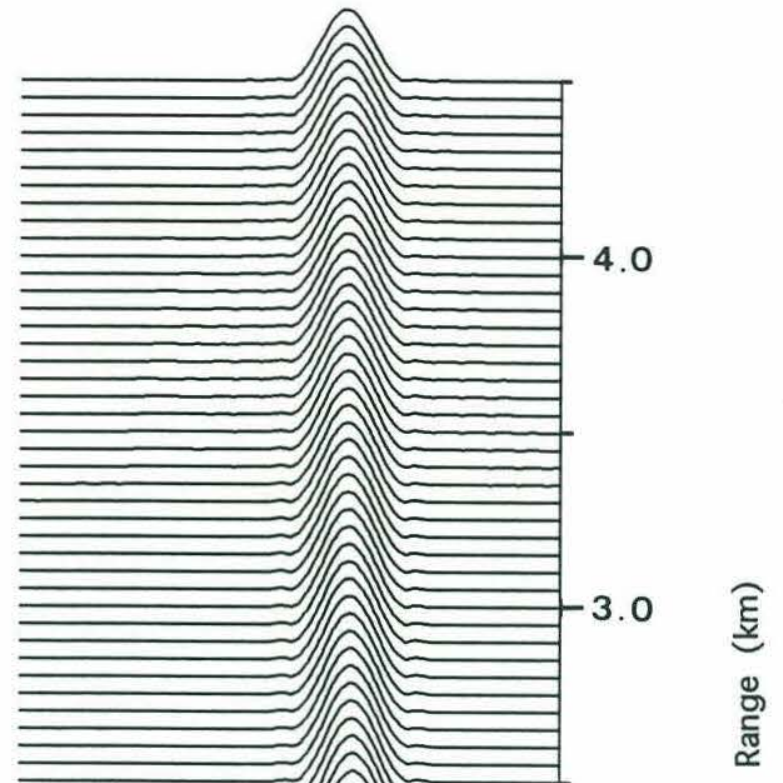

Mode 3
Mode 2

Figure 3-24, continued 


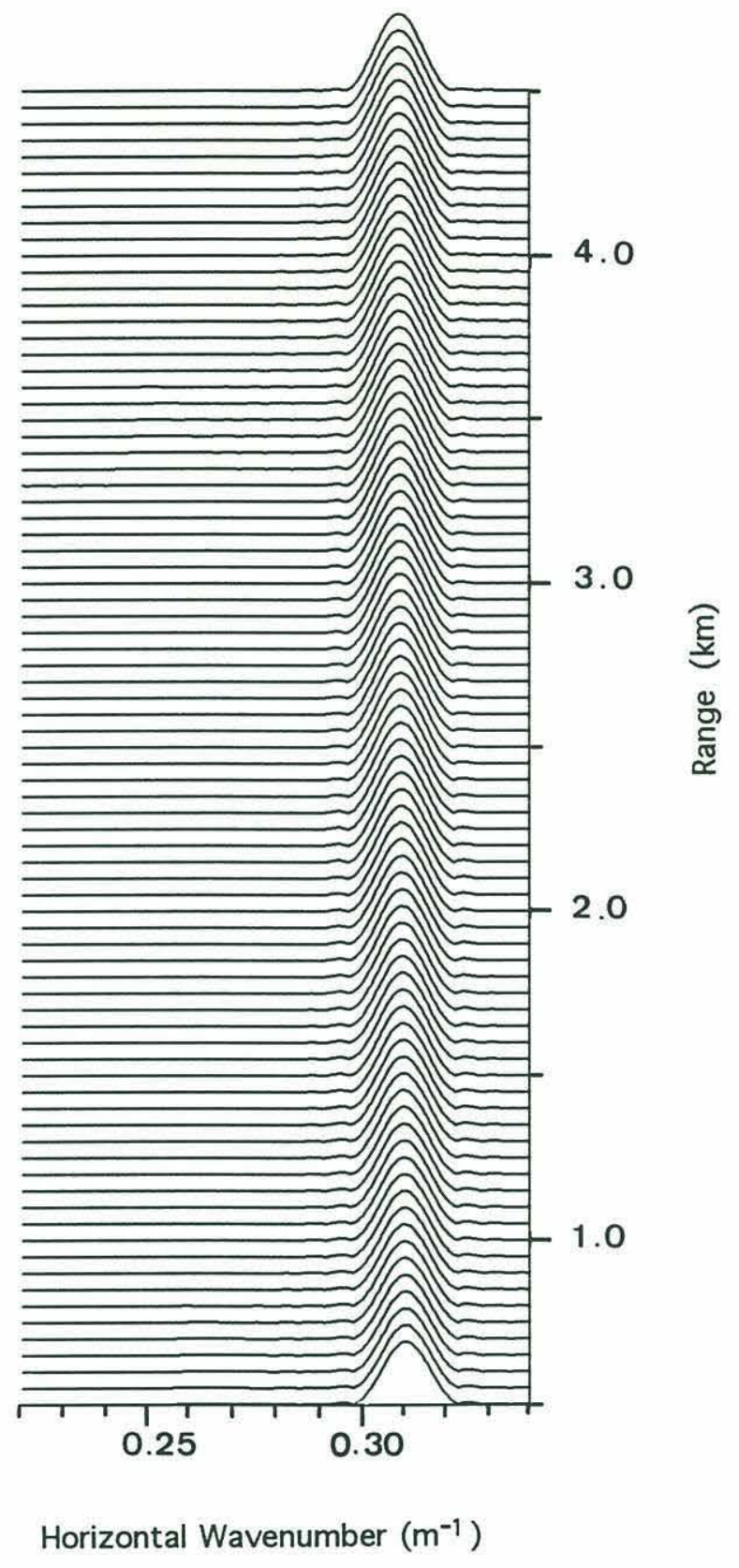

\section{Mode 1}

Figure 3-24, continued 


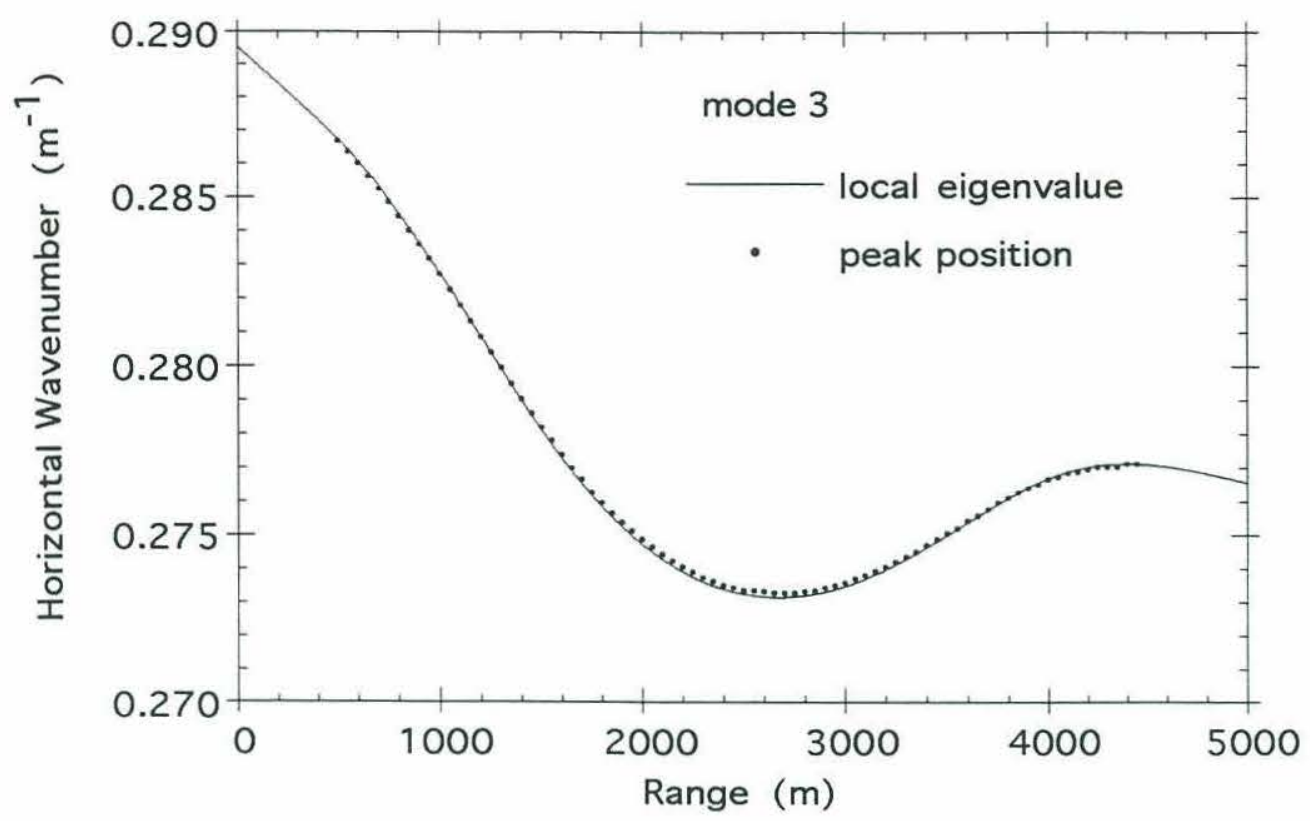

(a)

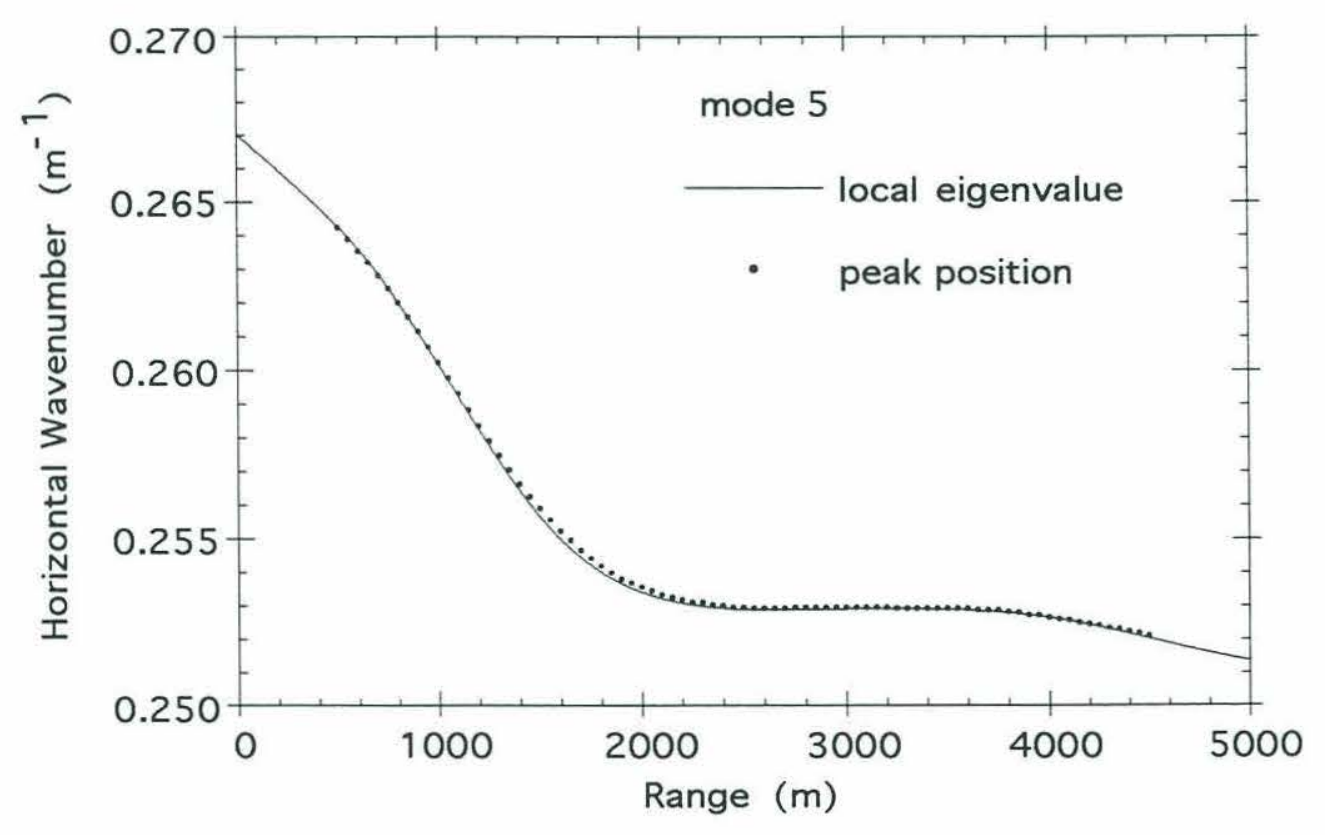

(b)

Figure 3-25: Comparison of local eigenvalue and modal peak trajectories in the spectrum in Figure 3-24: (a) mode 3 and (b) mode 5. 


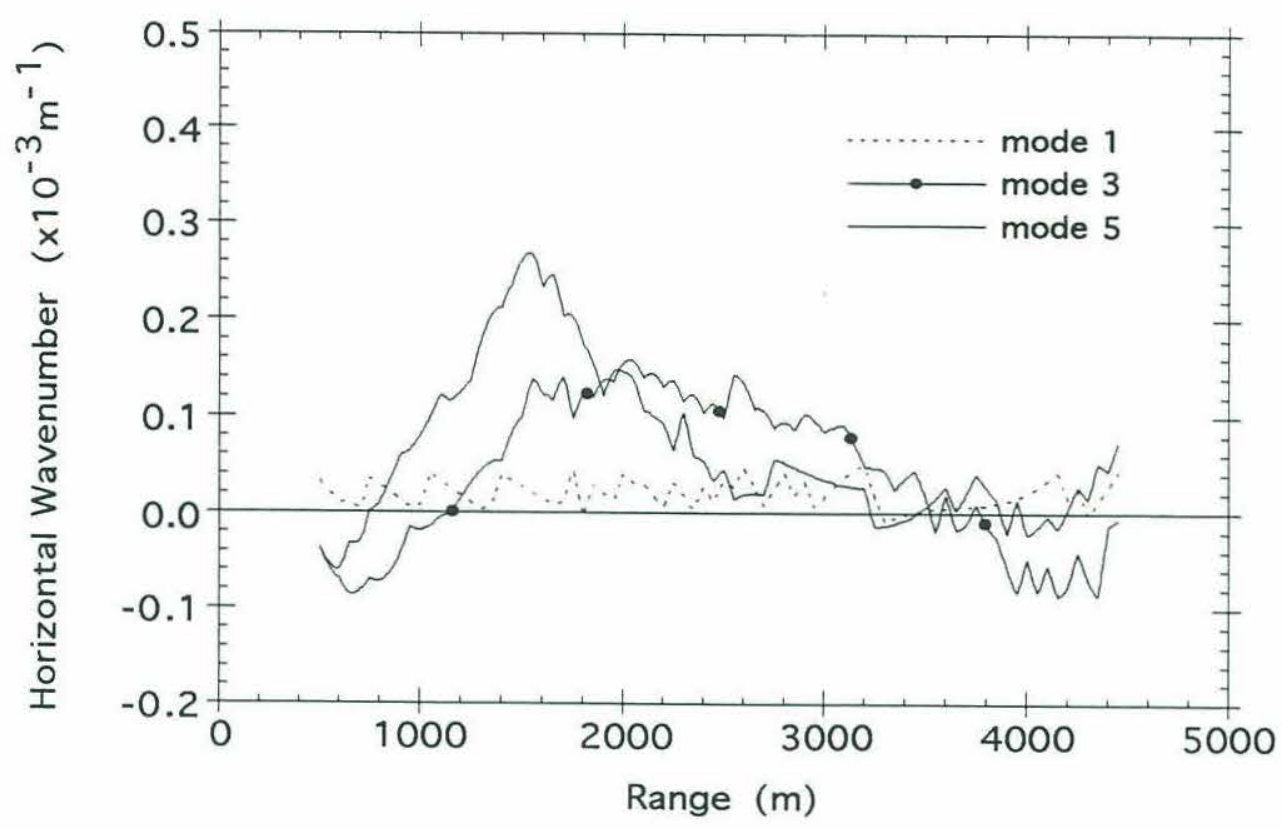

Figure 3-26: Comparison of the departures from the local eigenvalue for three different modes.

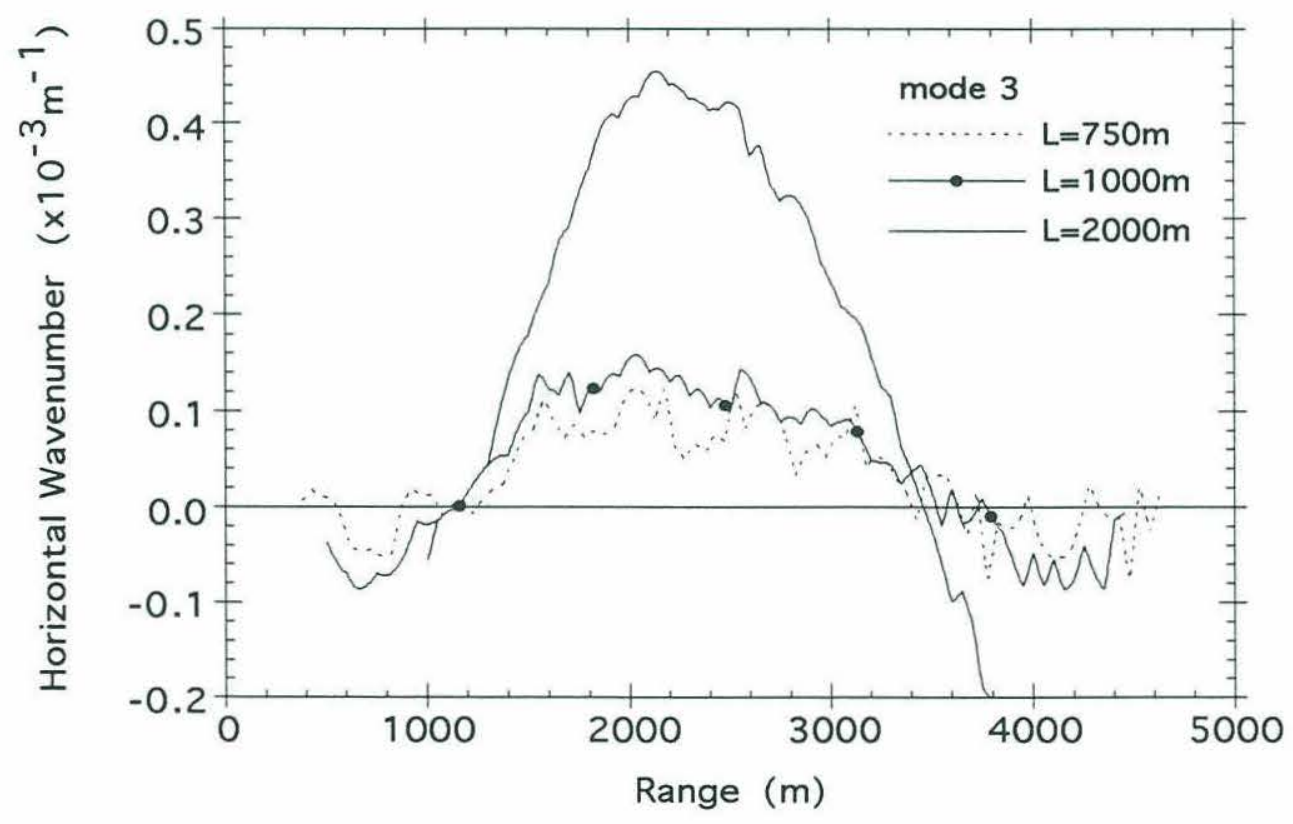

Figure 3-27: Comparison of the departures from the local eigenvalue for three different Hanning window lengths. 


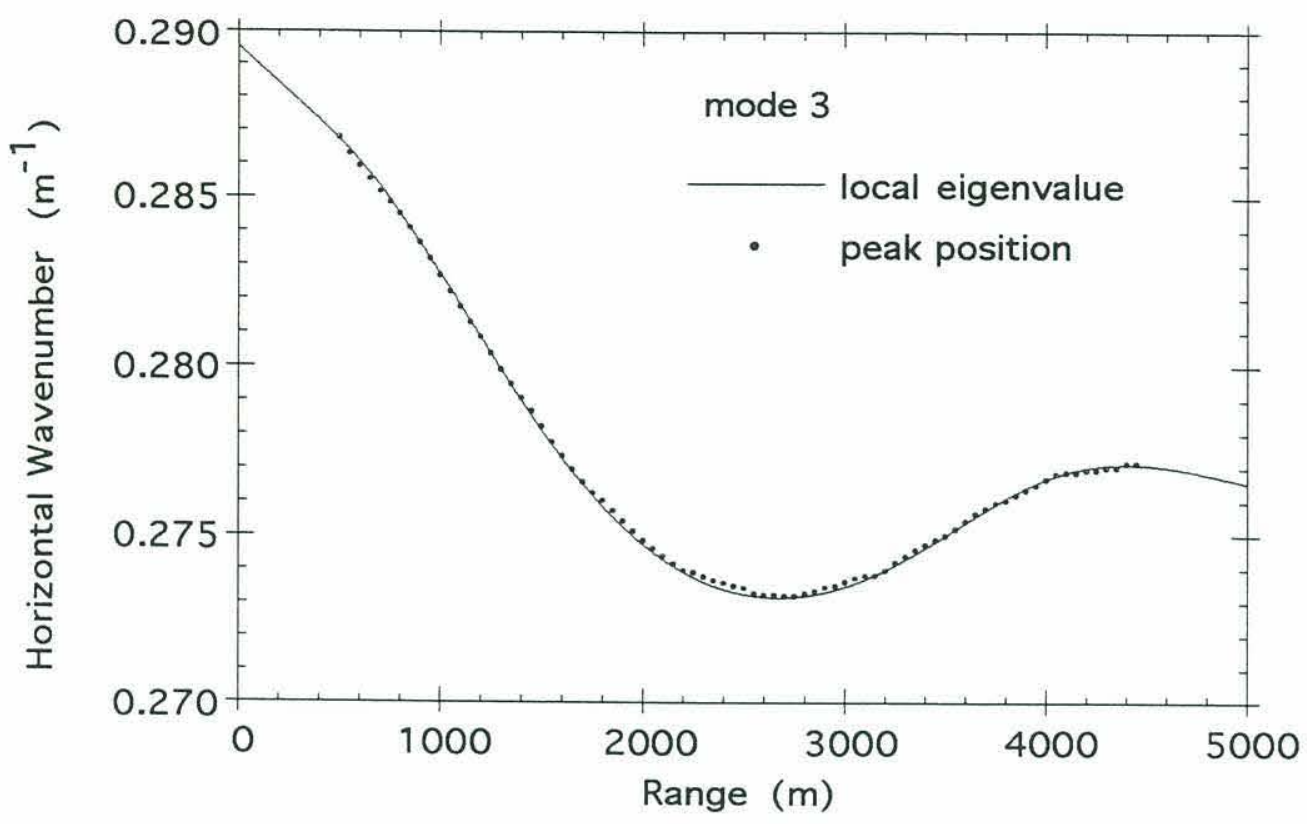

(a)

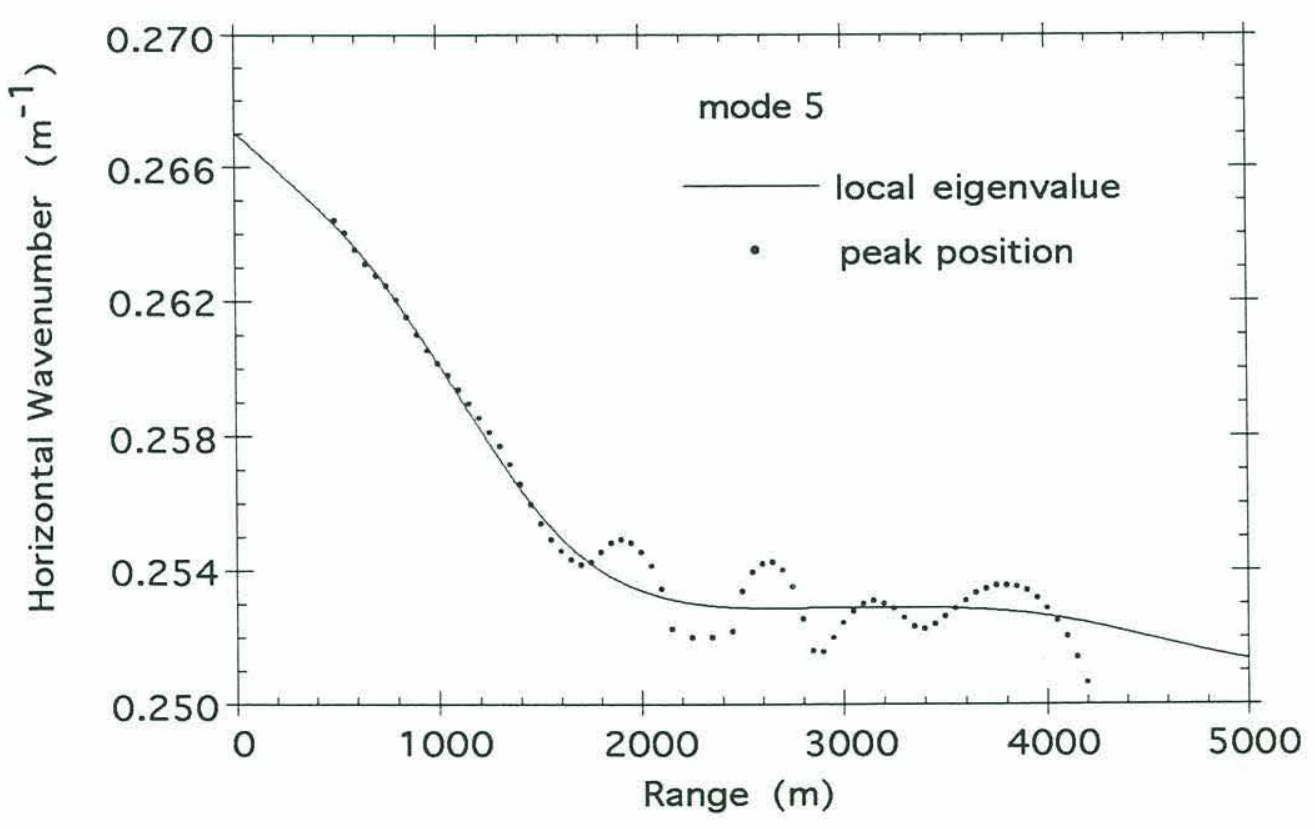

(b)

Figure 3-28: Comparison of exact local eigenvalue and modal peak trajectories in the spectrum separated by mode filtering with EDM. The pressure is simulated by using the first nine local modes in the model of a laterally inhomogeneous shallow water waveguide shown in Figures 3-9 to 3-14: (a) mode 3 and (b) mode 5. 


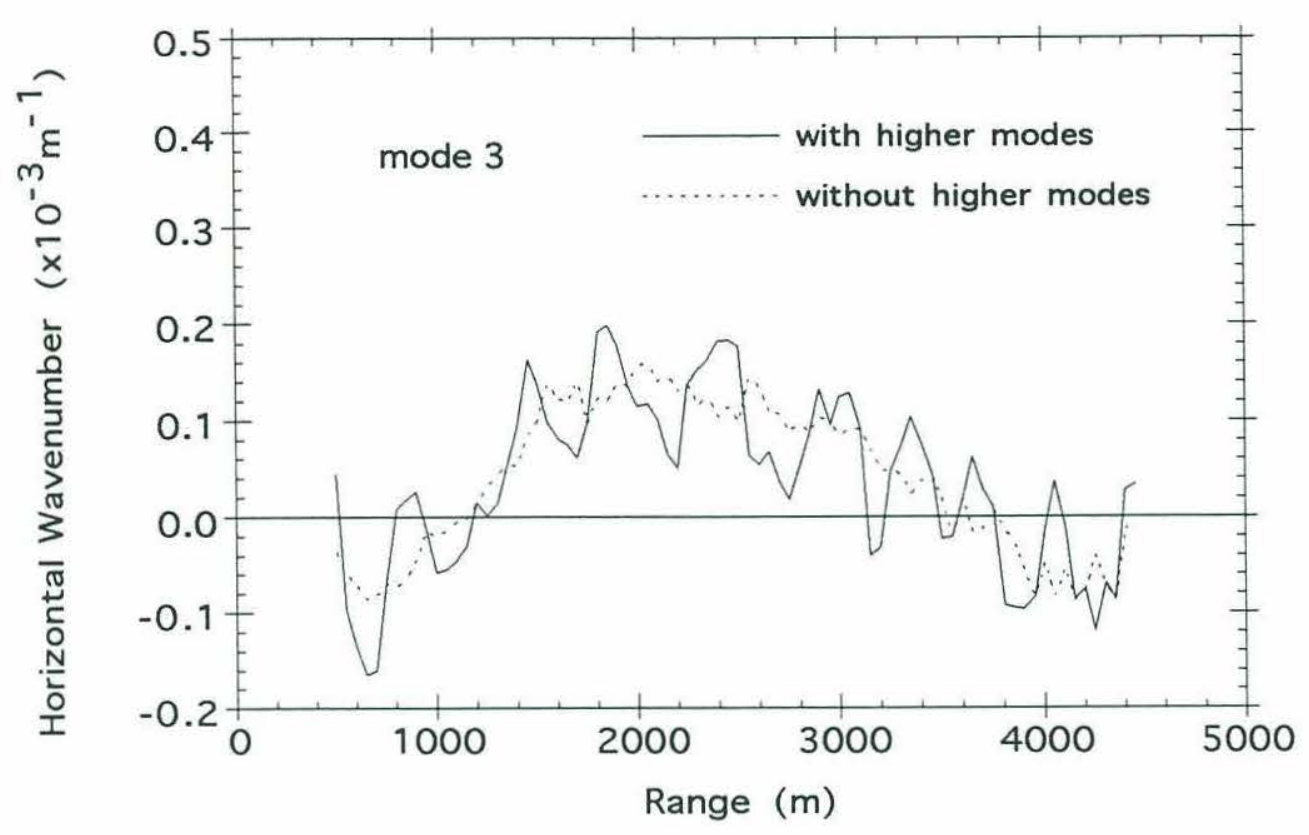

(a)

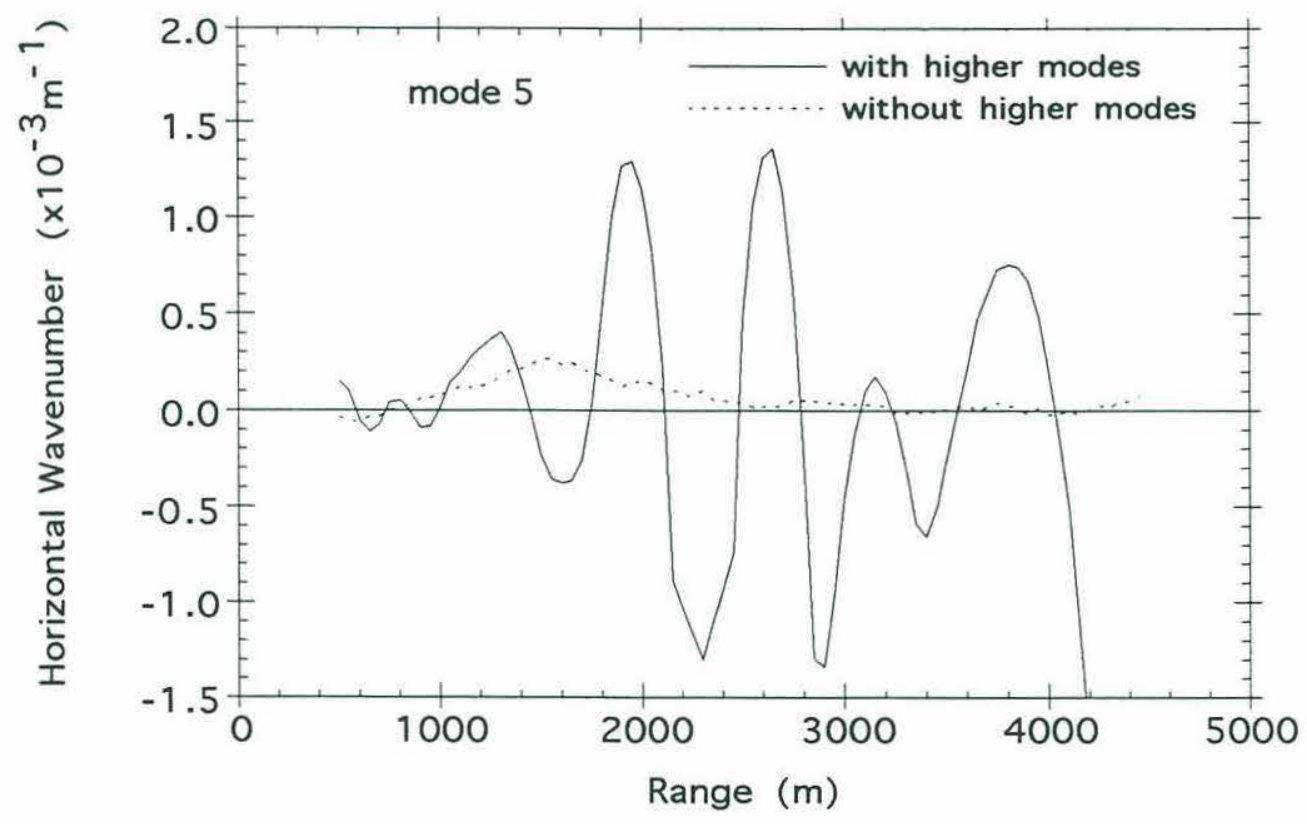

(b)

Figure 3-29: Comparison of the departures from the local eigenvalue for the field consisting of five modes and the field consisting of nine modes: (a) mode 3 and (b) mode 5. 
Table 3-1 : Eigenvalues of the matrix in Eq.(3-68), which were used for the EDM.

$\begin{array}{lc}i_{1} & \text { Eigenvalue }\left(\lambda_{i_{1}}\right) \\ 1 & 0.4507 \\ 2 & 0.4460 \\ 3 & 0.4357 \\ 4 & 0.1552 \\ 5 & 0.0158 \\ 6 & 0.0005\end{array}$

become extremely small, so that the generalized inverse matrix becomes unstable. (Note that these eigenvalues must be distinguished from the modal eigenvalues in Figures 3-16 and 3-18.) Hence, we utilized the EDM with $\breve{I}=6$ in Eq.(3-77) to obtain the stable generalized inverse matrix (Table 3-1). This result is shown in Figures 3-28(a) and (b) for modes 3 and 5 in the same manner as above. In Figure 3-29, the departure from the local eigenvalue is compared with the departure in Figure 3-26, which is obtained for the field without those higher modes. These results show that in the case of mode 3 , the effect of the higher modes can be eliminated by using EDM, whereas the fifth mode closest to the higher modes suffers from the interference with those modes.

At the end of this section let us study the effect of noise. In order to simulate the noisy data, we simply add white noise to the real and imaginary parts of the pressure field produced in Section 3.4.2. The white noise can be generated by using a Gaussian normal distribution routine. Note that the noise added at different receivers was taken to be independent from each other. Figure 3-30 shows examples of synthesized noisy pressure fields. The signal-to-noise ratio (SNR) was defined by using the range-averaged signal intensity. Here let us use the same mode filtering and Hankel transform as those used in 
Figure 3-24. Figure 3-31 shows examples of the resulting modal evolution. In Figures 332 the peak trajectories and the departure from the local eigenvalues are compared for the different SNR's. These figures indicate that mode 3 is the most sensitive to the noise and we cannot recognize the peak beyond a range of $3000 \mathrm{~m}$ for a SNR of $10 \mathrm{~dB}$. This is due to the fact that the attenuation of mode 3 is the largest in the present model (see Figure 316), so that mode 3 decreases most rapidly with range and has a larger influence from the noise. 

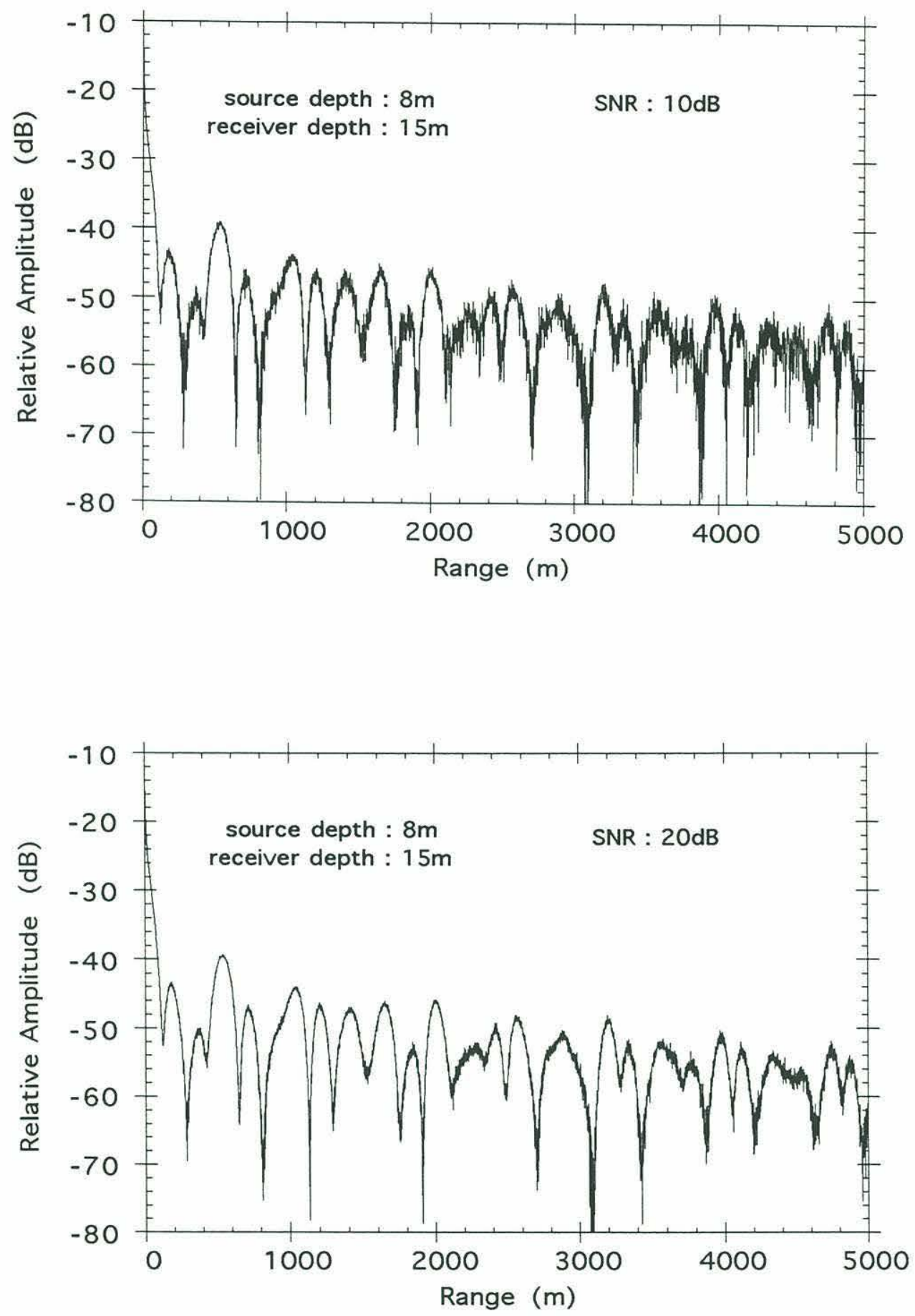

Figure 3-30: Synthesized noisy pressure fields. White noise has been added to the pressure field in Figure 3-19. 


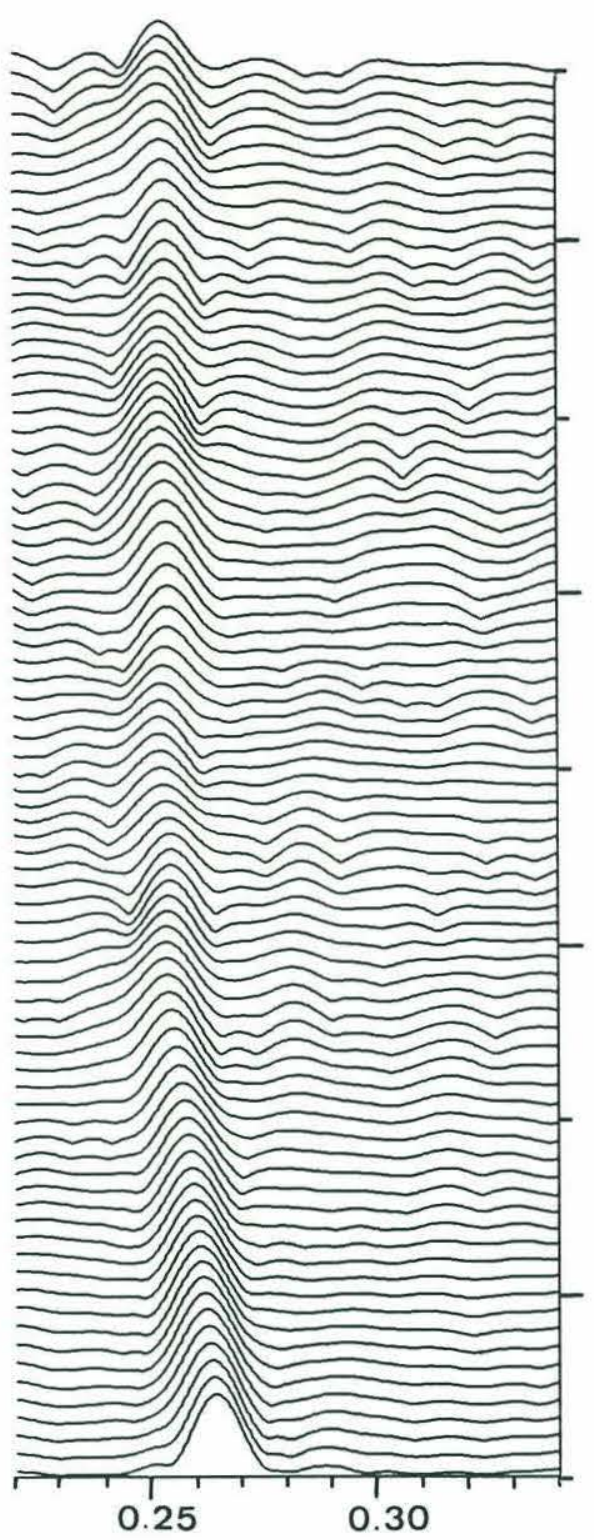

Horizontal Wavenumber $\left(\mathrm{m}^{-1}\right)$

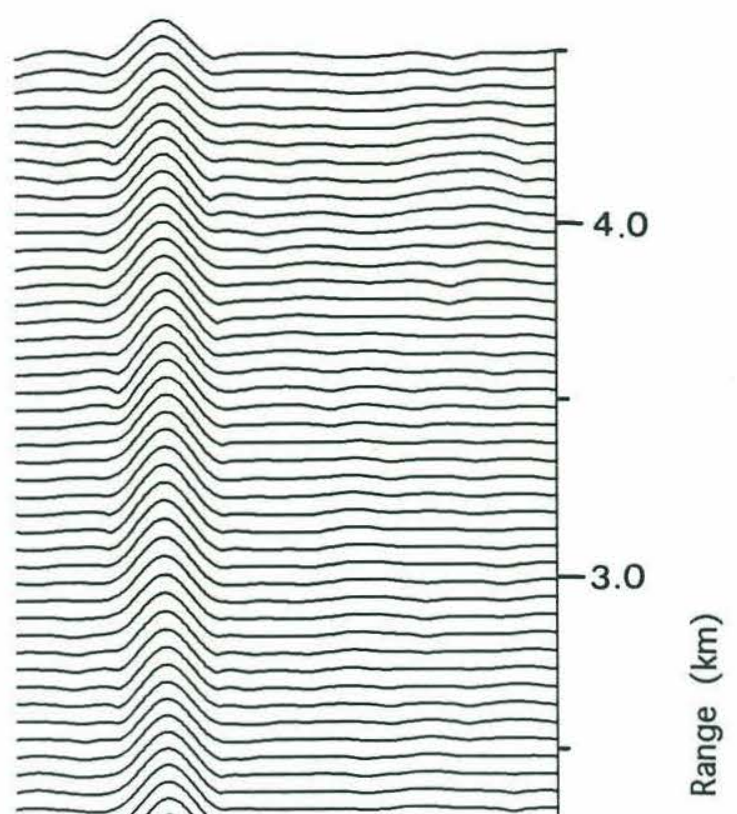

SNR $10 \mathrm{~dB}$

SNR 20dB

Figure 3-31: Range-dependent evolution of the wavenumber spectrum (mode 5) obtained by applying the same mode filtering and Hankel transform as those in Figure 3-24 to the noisy pressure fields. 

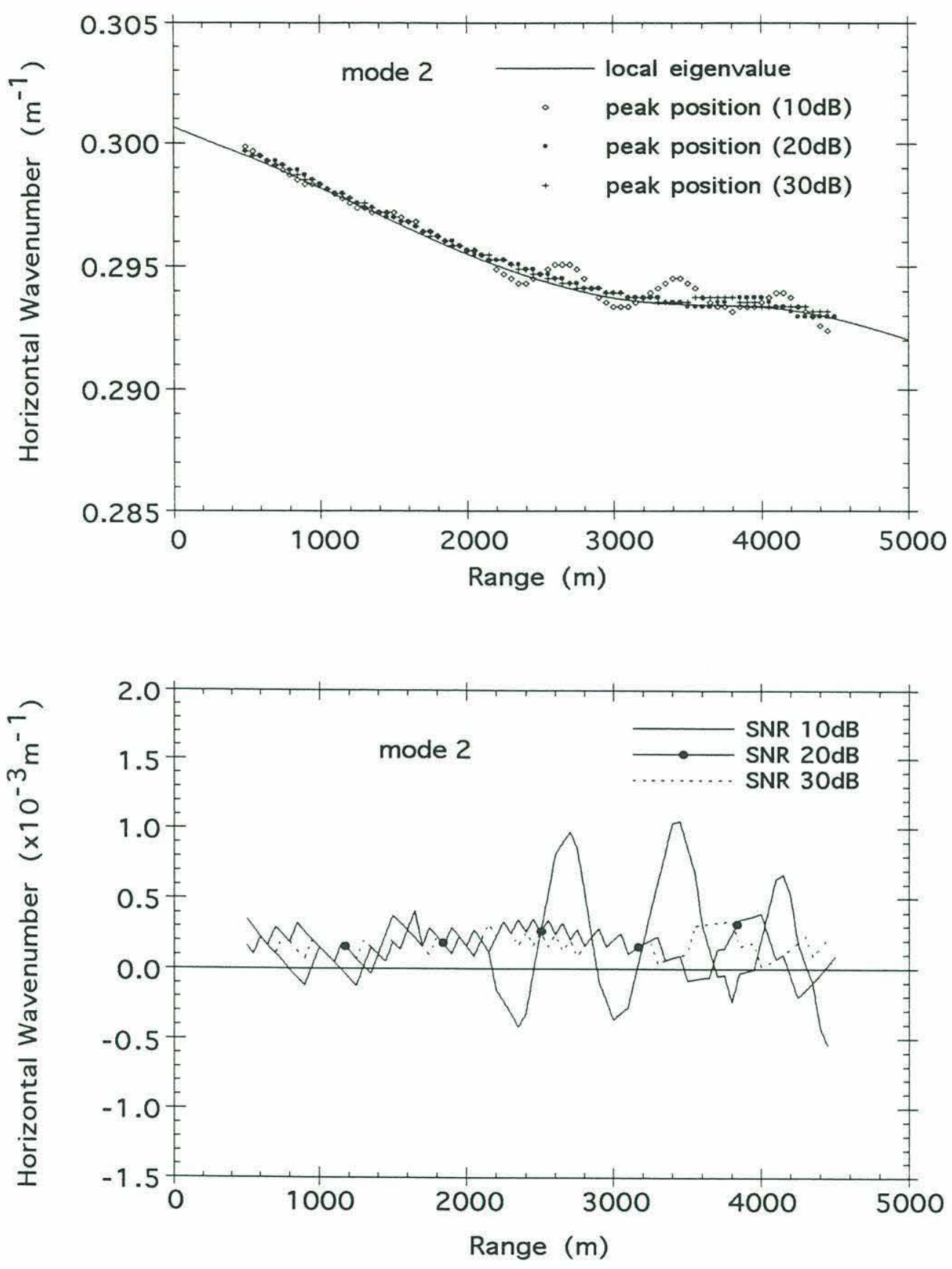

(a)

Figure 3-32: Comparison of local eigenvalue and peak trajectories for different SNR's (upper), and departure from the local eigenvalue (lower). (a) mode 2 , (b) mode 3 , and (c) mode 5. 

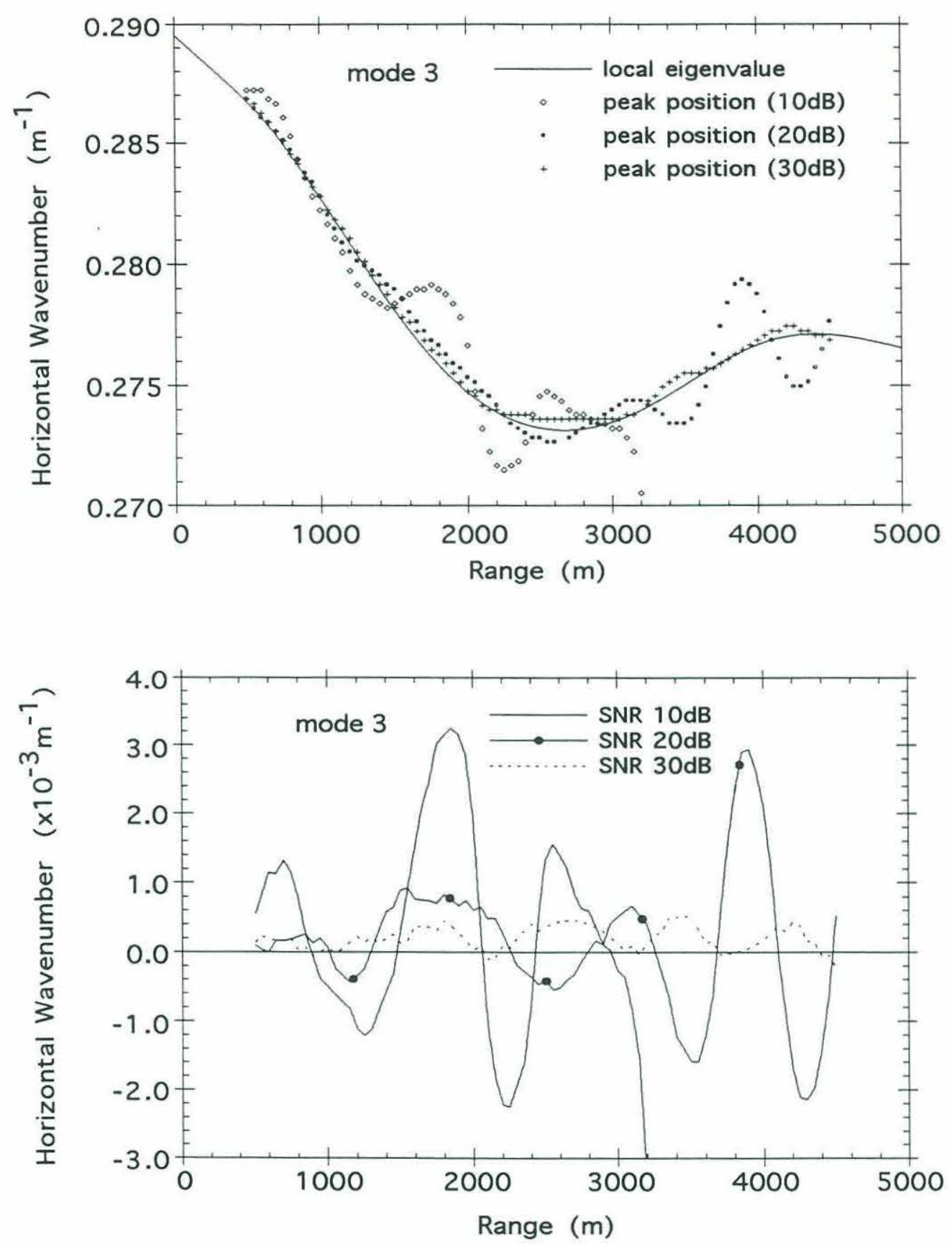

(b)

Figure 3-32, continued. 

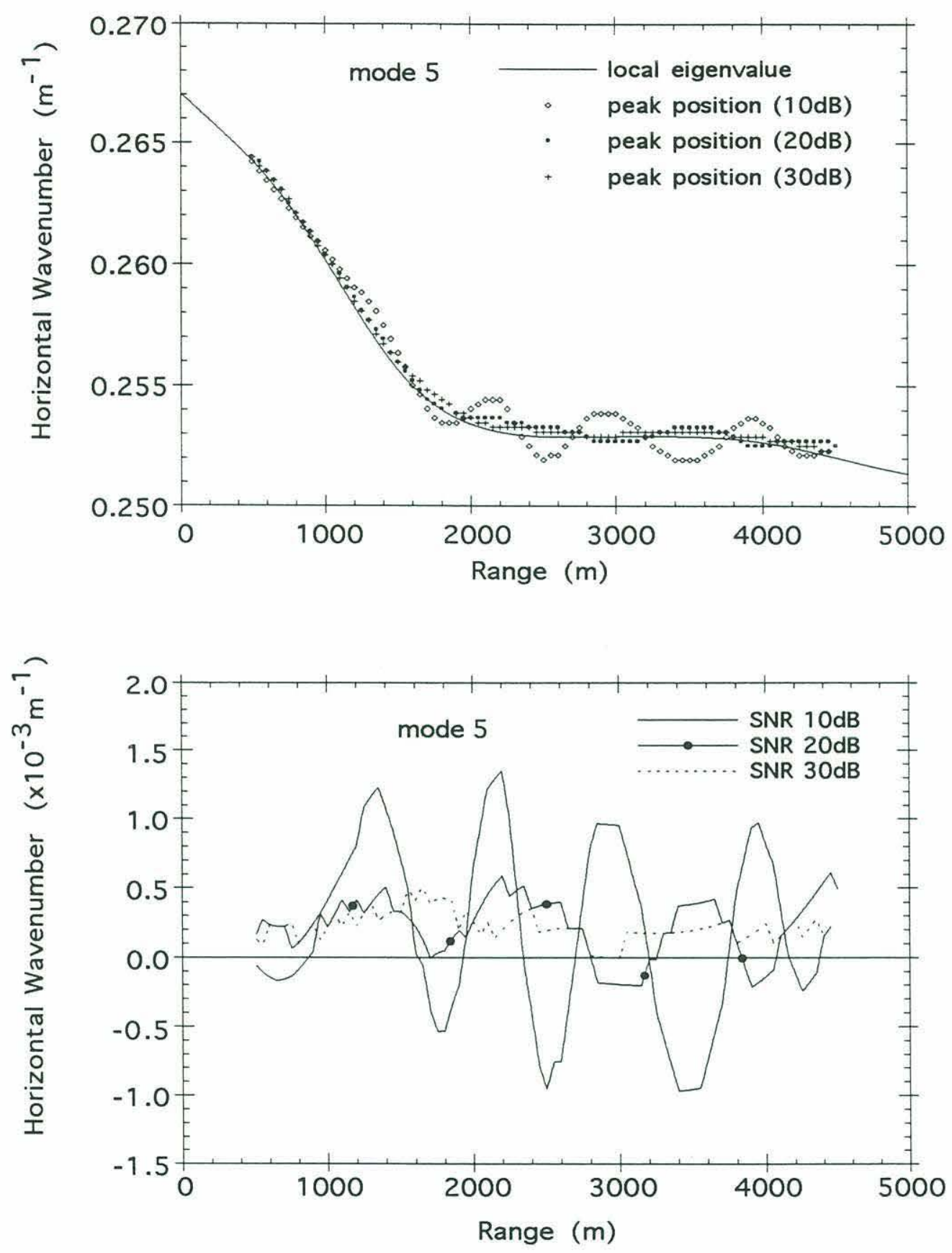

(c)

Figure 3-32, continued. 


\subsection{Summary}

In an attempt to extract local eigenvalues from the pressure field in a laterally inhomogeneous waveguide, the asymptotic Hankel transform with a sliding window is utilized in this Chapter.

In Section 3.1, we reviewed the definition of the Hankel transform and then, in the case of a horizontally stratified waveguide, it was shown that the eigenvalues of the normal modes can be accurately detected by applying a zero-order asymptotic Hankel transform to the pressure field in the waveguide.

In Section 3.2, a sliding window was introduced into the zero-order asymptotic Hankel transform to detect the local eigenvalues in a laterally varying waveguide. In order to analyze the errors in this method, we applied the asymptotic Hankel transform with a sliding window to the pressure field expressed by the adiabatic mode sum and examined analytically the effect of range dependence on the local eigenvalue estimates. In a single mode situation, it was found that the departure of the peak position in the output spectrum from the local eigenvalue depends on both the second derivative of the local eigenvalue and the window length and type.

In addition to this error, another type of departure from the local eigenvalue is induced by the interference with the sidelobes of adjacent modes. To reduce the latter type of error, the separation of modes prior to the application of the Hankel transform with a sliding window is desirable. In order to accomplish this, we exploited mode filtering by incorporating data from a fixed vertical array of receivers in Section 3.3. When this filtering process becomes unstable due to the involvement of a singular matrix, it was demonstrated that the eigenvector decomposition method (EDM) and the stabilized least-mean-square method (SLMS) are useful in providing reliable mode separation.

In Section 3.4, we applied the asymptotic Hankel transform with a sliding window and mode filtering to a pressure field which was simulated numerically by using adiabatic mode theory in a model of a laterally inhomogeneous shallow water waveguide. The 
results indicated that the use of mode filtering improved the detection of the local eigenvalues. When the field includes higher modes, it is confirmed that the EDM is useful in providing a stable results for mode separation.

In order to address the case of a 3-D varying environment, results obtained in this chapter will be extended in the next chapter. 


\section{Chapter 4 \\ Analysis of the Effect of Horizontal Refraction \\ on the Hankel Transform}

In the previous chapter, we assumed the waveguide to be cylindrically symmetric around a fixed source or receiver array, so that the sound propagates in the radial direction and does not suffer from horizontal refraction. In general, however, we must take into consideration the effect of horizontal refraction when a medium with bottom sediments varies arbitrarily but gradually in the horizontal direction so that the pressure field does not remain symmetric.

In this chapter, we will explore a method for determining local eigenvalues for noncylindrically symmetric fields. In the first section, the problem of horizontal refraction is raised. In Section 4.2, we explore the use of a general Hankel transform with a sliding window based on the scheme discussed in Chapter 3. In Section 4.3, an alternative representation of a 2-D Fourier transform with a sliding window is derived and we examine the effect of horizontal refraction. In Section 4.4, the results obtained in Sections 4.2 and 4.3 are numerically studied by applying them to a pressure field that is simulated by using the horizontal ray method. In Section 4.5 , a way for effective measurement in a 3-D varying environment is considered.

\subsection{Problem definition}

When we utilize the Nx2D method in a 3-D varying waveguide, the pressure field can be synthesized by using only the sound speed profile and bottom sediment conditions along a radial, just as if the medium were symmetric. The simulated field can provide a 
good approximation to the pressure field and actually includes horizontal refraction to some extent. This is due to the following approximate relation:

$$
\int_{(0,0)}^{(x, y)} \kappa_{n}(x(s), y(s)) d s \sim \int_{0}^{r} \kappa_{n}\left(r, \theta_{0}\right) d r
$$

where the integral on the left-hand side is executed along the horizontal ray path $s$ of the mode propagating from the origin to $(x, y)$, whereas the integral on the right-hand side is taken simply along the fixed radial ( $\theta_{0}$ direction).

In the inverse problem for detecting eigenvalues from a given pressure field, however, we are not able to take advantage of Eq.(4-1); that is, to apply the asymptotic Hankel transform with the sliding window in Eq.(3-30) to a non-cylindrically symmetric field along some fixed radial by assuming Eq.(4-1). If we do this, then the wavenumber of the peaks detected by this transform is only the component projected on to this radial direction (Figure 4-1) and, consequently, we would always underestimate the local eigenvalues. This is because what is accounted for by the transform is the rate of spatial change of phase along the radial, not the accumulated phase shown in Eq.(4-1).

Thus the key to this problem is how to invert for the horizontal refraction angle at each observation point. If this angle were known a priori, then we could compensate for the underestimated wavenumber discussed above by using this angle. For example, assume a 3-D varying waveguide such that the horizontal refraction of modes is dominated only by bathymetric change. We can then predict the refraction angle by means of the horizontal ray technique. In shallow water, however, this assumption does not always hold, especially for higher modes in the lower frequency region. Namely, those modes are often more sensitive to the variation of the sediment structure and properties rather than the bathymetric change in the propagation process [79]. 


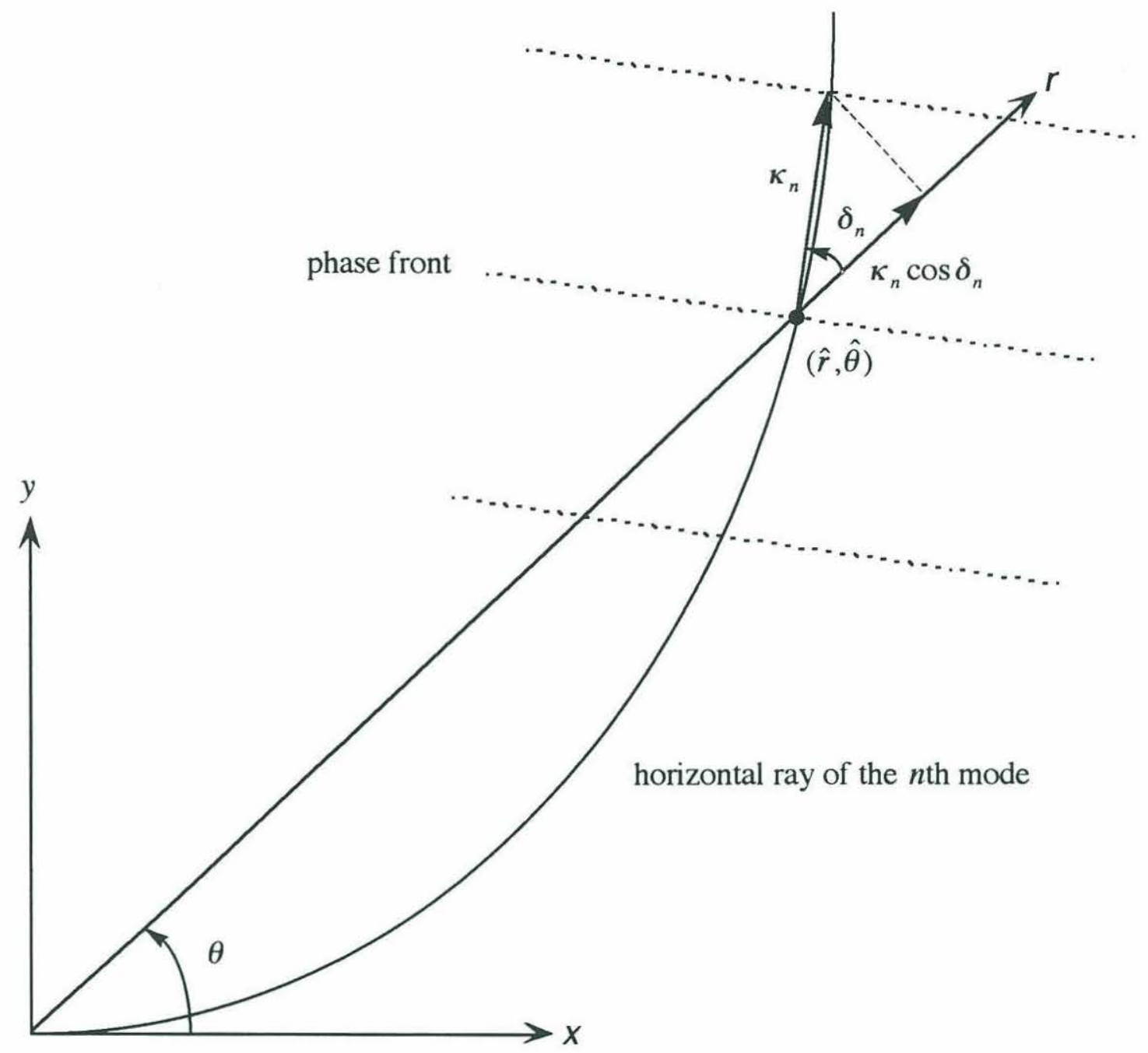

Figure 4-1: Schematic illustration of the phase front passing the observing point $(\hat{r}, \hat{\theta})$ and wavenumber component in the radial direction. 
Therefore, in order to obtain accurate local eigenvalues in a non-cylindrically symmetric environment, we must use a 2-D Fourier transform or general Hankel transform.

Since the region of the pressure field to be examined is an intermediate range (e.g., $r<R_{I} \sim 10 \mathrm{~km}$ ), the phase front associated with a mode is primarily spreading out in the radial direction. Also this phase front does not change its direction suddenly due to the adiabatic assumption. Thus if we use a cylindrical coordinate system, the direction of this modal evolution can be described along a radial plus a perturbation. With this viewpoint, the measurement along the radial direction, as carried out in the cylindrically symmetric case, can be the most effective way to provide the pressure data for determining the dominant variation in the local eigenvalue. Hence, based on this notion, we will continue to use a cylindrical coordinate system in the following sections.

\subsection{General Hankel transform with a sliding window}

In this section, we will explore a method for obtaining local eigenvalues for a noncylindrically symmetric field. In this case, we have to start from the 2-D Fourier transform in the form of Eq.(3-5) to obtain the spatial spectrum in terms of the cylindrical coordinates $\left(k_{r}, \varphi\right)$. As discussed in Chapter 3 , a window function may be incorporated into Eq.(3-5) in order to obtain the local character of the wavenumber spectrum:

$$
g\left(k_{r}, \varphi ; \hat{r}, \hat{\theta}, z\right)=\frac{1}{2 \pi} \int_{0}^{\infty} d r w_{L}(r ; \hat{r}) r \int_{0}^{2 \pi} d \theta v_{\Omega}(\theta ; \hat{\theta}) p(r, \theta, z) e^{-i k, \cos (\theta-\varphi)}
$$

where $\tilde{g}$ in Eq.(3-5) has been replaced by $g$ with the use of the relation in Eq.(3-10). Here, as shown in Figure 4-2, the window is given by the product of $w_{L}(r ; \hat{r})$ and $v_{\Omega}(\theta ; \hat{\theta})$; the former is a window function for the range direction with a center position of $\hat{r}$ and a length of $L$ (as in the symmetric case), and the latter is a window function for the azimuthal direction with a center direction of $\hat{\theta}$ and an angular width of $\Omega$. By 


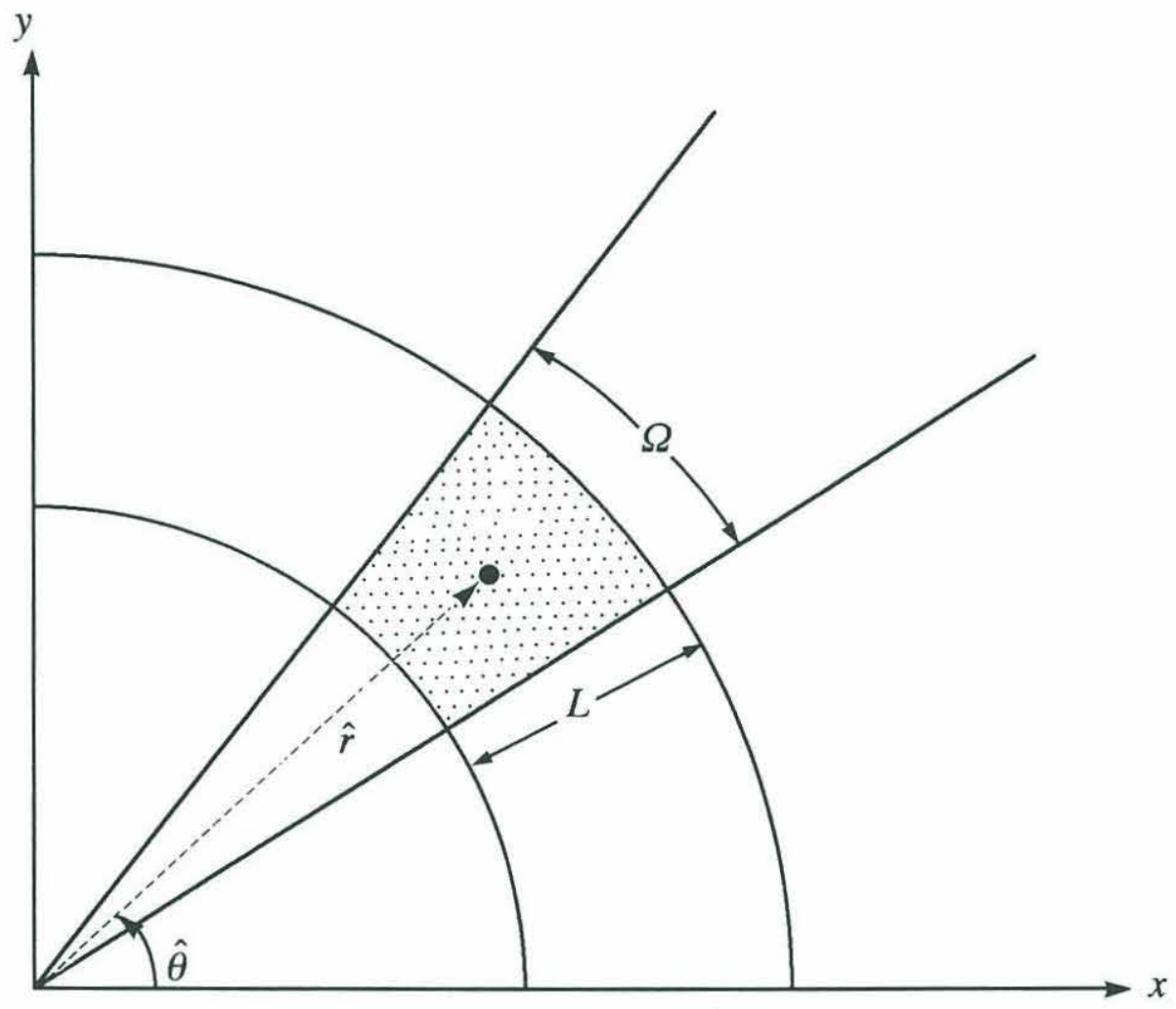

Figure 4-2: Schematic illustration of the 2-D window defined by $w_{L}(r ; \hat{r}) \times v_{\Omega}(\theta ; \hat{\theta})$. 
choosing a small width for $\Omega$, we can reduce the variation in the propagation direction of the phase front associated with $\kappa_{n}$ inside this window, as long as the range of interest is in $r<R_{I}$. On the other hand, the minimum length of $L$ is restricted in order to attain the required resolution in the output spectrum in $k_{r}$.

\subsubsection{Derivation of the general Hankel transform with a sliding window}

Now, in order to derive a general Hankel transform, the exponential term in Eq.(4-2) can be rewritten using the generating function for the Bessel function [57]:

$$
e^{i k_{r} r \sin \theta}=\sum_{n=-\infty}^{\infty} J_{n}\left(k_{r} r\right) e^{i n \theta}
$$

where $J_{n}\left(k_{r} r\right)$ is the $n$th order Bessel function. After converting the sine function to a cosine function by adding $\pi / 2$ to its argument, we substitute Eq.(4-3) into Eq.(4-2), which yields

$$
g\left(k_{r}, \varphi ; \hat{r}, \hat{\theta}, z\right)=\frac{1}{2 \pi} \int_{0}^{\infty} d r w_{L}(r ; \hat{r}) r \int_{0}^{2 \pi} d \theta v_{\Omega}(\theta ; \hat{\theta}) p(r, \theta, z)\left[\sum_{n=-\infty}^{\infty} J_{n}\left(k_{r} r\right) e^{i n\left(\varphi-\theta-\frac{\pi}{2}\right)}\right] .
$$

By changing the order of summation and integration with respect to $\theta$, it follows that

$$
g\left(k_{r}, \varphi ; \hat{r}, \hat{\theta}, z\right)=\int_{0}^{\infty} d r w_{L}(r ; \hat{r}) r \sum_{n=-\infty}^{\infty} C_{n}(r ; \hat{\theta}, z) J_{n}\left(k_{r} r\right) e^{i n\left(\varphi-\frac{\pi}{2}\right)}
$$

where $C_{n}(r)$ represents the Fourier coefficient at range $r$, which is defined by

$$
C_{n}(r ; \hat{\theta}, z)=\frac{1}{2 \pi} \int_{0}^{2 \pi} d \theta v_{\Omega}(\theta ; \hat{\theta}) p(r, \theta, z) e^{-i n \theta}
$$


Owing to the window function $v_{\Omega}(\theta ; \hat{\theta})$, the pressure field required for Eq.(4-6) is limited in the azimuthal width $\Omega$. Here attention should be paid to the size of $\Omega$. Namely, this cannot be reduced as much as desired, depending on the type of the window $v_{\Omega}(\theta ; \hat{\theta})$. If $v_{\Omega}(\theta ; \hat{\theta})$ has a discontinuity such as a rectangular window, then the Fourier series representation for this function has an error due to the Gibb's phenomenon [70]. Hence, in this sense, the Hanning window is preferable to a rectangular window.

Since the range of interest is far enough to satisfy $k_{r} r \gg 1$, we can employ the asymptotic form for the Bessel function as done in Chapter 3. To do this, we use the identity:

$$
J_{n}\left(k_{r} r\right)=\frac{1}{2}\left[H_{n}^{(1)}\left(k_{r} r\right)+H_{n}^{(2)}\left(k_{r} r\right)\right]
$$

with the asymptotic form for the Hankel function [57]:

$$
\begin{aligned}
& H_{n}^{(1)}\left(k_{r} r\right) \sim \sqrt{\frac{2}{\pi k_{r} r}}\left[\wp_{1}+i \wp_{2}\right] e^{i\left(k_{r}-\frac{\pi}{4}\right)}, \\
& H_{n}^{(2)}\left(k_{r} r\right) \sim \sqrt{\frac{2}{\pi k_{r} r}}\left[\wp_{1}-i \wp_{2}\right] e^{-i\left(k_{r} r-\frac{\pi}{4}\right)},
\end{aligned}
$$

where

$$
\begin{aligned}
& \wp_{1}=1-\frac{\left(4 n^{2}-1^{2}\right)\left(4 n^{2}-3^{2}\right)}{2 !\left(8 k_{r} r\right)^{2}}+\cdots, \\
& \wp_{2}=\frac{4 n^{2}-1^{2}}{8 k_{r} r}-\frac{\left(4 n^{2}-1^{2}\right)\left(4 n^{2}-3^{2}\right)\left(4 n^{2}-5^{2}\right)}{3 !\left(8 k_{r} r\right)^{3}}+\cdots .
\end{aligned}
$$

In the case of cylindrically symmetric fields, we used only the initial term in the asymptotic form of the zero-order Hankel function as shown in Eq.(3-17) and Eq.(3-18), 
while here the higher order terms in Eq.(4-9) cannot be simply neglected because $n$ goes to infinity in the summation in Eq.(4-5).

The pressure field due to a point source at $r=0$ is dominated by an outgoing wave in the region of interest $\left(r<R_{I}\right)$, even when subjected to horizontal refraction. Thus, when we substitute Eq.(4-7) with Eq.(4-8) and Eq.(4-9) into Eq.(4-5), the terms including $e^{i k r}$ do not substantially contribute to the integral in Eq.(4-5) and can be dropped out of this equation, which yields

$$
\begin{aligned}
g\left(k_{r}, \varphi ; \hat{r}, \hat{\theta}\right) \sim \frac{e^{i \frac{\pi}{4}}}{\sqrt{2 \pi k_{r}}} \int_{0}^{\infty} d r w_{L}(r ; \hat{r}) \sqrt{r} e^{-i k_{r} r} \\
\times \sum_{n=-\infty}^{\infty} C_{n}(r ; \hat{\theta})\left[1-i \frac{\left(4 n^{2}-1^{2}\right)}{8 k_{r} r}-\frac{\left(4 n^{2}-1^{2}\right)\left(4 n^{2}-3^{2}\right)}{2 !\left(8 k_{r} r\right)^{2}}\right. \\
\left.+i \frac{\left(4 n^{2}-1^{2}\right)\left(4 n^{2}-3^{2}\right)\left(4 n^{2}-5^{2}\right)}{3 !\left(8 k_{r} r\right)^{3}}+\cdots\right] e^{i n \varphi} .
\end{aligned}
$$

For simplicity, the functional notation for depth is omitted in Eq.(4-10) and also in the following equations.

By keeping the leading term in the numerator of each fraction within the bracket when summing over $n$, Eq.(4-10) can be approximated by

$$
\begin{aligned}
g\left(k_{r}, \varphi ; \hat{r}, \hat{\theta}\right) \sim & \frac{e^{i \frac{\pi}{4}}}{\sqrt{2 \pi k_{r}}} \int_{0}^{\infty} d r w_{L}(r ; \hat{r}) \sqrt{r} e^{-i k, r} \\
& \times \sum_{n=-\infty}^{\infty} C_{n}(r ; \hat{\theta})\left[1+\frac{-i n^{2}}{2 k_{r} r}+\frac{1}{2 !}\left(\frac{-i n^{2}}{2 k_{r} r}\right)^{2}+\frac{1}{3 !}\left(\frac{-i n^{2}}{2 k_{r} r}\right)^{3}+\cdots\right] e^{i n \varphi} .
\end{aligned}
$$

Noticing that the inside of the bracket is a form of a Taylor series for $e^{-i \frac{n^{2}}{2 k r}}$, we obtain the following form: 


$$
g\left(k_{r}, \varphi ; \hat{r}, \hat{\theta}\right) \sim \frac{e^{i \frac{\pi}{4}}}{\sqrt{2 \pi k_{r}}} \int_{0}^{\infty} d r w_{L}(r ; \hat{r}) \sqrt{r} e^{-i k_{r} r} \sum_{n=-\infty}^{\infty} C_{n}(r ; \hat{\theta}) e^{-i \frac{n^{2}}{2 k_{r}}} e^{i n \varphi}
$$

The right-hand side of Eq.(4-12) can be evaluated numerically in principle, but realistically this requires too much computational effort. This is due to the factor $k_{r} r$ located in the denominator in $e^{-i \frac{n^{2}}{2 k r}}$, so that we cannot simply make use of the FFT when performing the integral with respect to $r$, as done in Chapter 3.

Therefore we will examine Eq.(4-12) analytically in order to evaluate it in an approximate manner. In Section 4.2.2, we expand Eq.(4-12) by using a differential operator with respect to the azimuthal angle in order to study the relationship between the zero-order Hankel transform in Eq.(3-30) and the general Hankel transform in Eq.(4-12). On the other hand, in Section 4.2.3, we derive an approximate form of this transform by resorting to the stationary phase method. Based on the analysis of the horizontal refraction effect with the use of this approximate form, we will explore a method for determining the horizontal refraction angle.

\subsubsection{Relationship to the zero-order Hankel transform}

Unless the term $e^{-i \frac{n^{2}}{2 k_{r}}}$ is included in Eq.(4-12), the sum over $n$ stands for the Fourier series for $p(r, \varphi)$, so that this representation reduces to the asymptotic form of a zeroorder Hankel transform given by Eq.(3-30) with replacement of $\theta$ by $\varphi$. As a special case, if $p(r, \theta)$ has no angular dependence such as in the cylindrically symmetric case, we have $C_{0}=p(r, \hat{\theta})$ and $C_{n}=0(n \neq 0)$ from Eq.(4-6) and thus Eq.(4-12) again reduces to Eq. $(3-30)$. Therefore we see that the term $e^{-i \frac{n^{2}}{2 k, r}}$ is a key factor for resolving the horizontal refraction effect in this transform. Actually, as observed from the fact that $e^{-i \frac{n^{2}}{2 k, r}}$ has both $n$ - and $k_{r}$-dependence, this term contains the interaction of transforms in the radial and angular directions. 
It is well known that the index $n$ in the Fourier series corresponds to the operator $-i \partial / \partial \theta$, i.e., in general:

$$
\sum_{n} n^{j} C_{n} e^{i n \theta}=\left(\frac{\partial}{i \partial \theta}\right)^{j} \sum_{n} C_{n} e^{i n \theta} \quad(j: \text { arbitrary integer }) .
$$

If one applies these properties in Eq.(4-13) to Eq.(4-12), then it follows that

$$
\left.g\left(k_{r}, \varphi ; \hat{r}, \hat{\theta}\right) \sim \frac{e^{i \frac{\pi}{4}}}{\sqrt{2 \pi k_{r}}} \int_{-\infty}^{\infty} d r w_{L}(r ; \hat{r}) \sqrt{r} e^{-i k k_{r}}\left[e^{i \frac{1}{2 k_{r}}\left(\frac{\partial}{\partial \theta}\right)^{2}} p(r, \theta)\right]\right|_{\theta=\varphi},
$$

or, using Eq.(4-11),

$$
\begin{aligned}
g\left(k_{r}, \varphi ; \hat{r}, \hat{\theta}\right) \sim \frac{e^{i \frac{\pi}{4}}}{\sqrt{2 \pi k_{r}}} \int_{-\infty}^{\infty} d r w_{L}(r ; \hat{r}) \sqrt{r} e^{-i k, r} \\
\quad \times\left[p(r, \varphi)+\left.\frac{i}{2 k_{r} r}\left(\frac{\partial^{2} p}{\partial \theta^{2}}\right)\right|_{\theta=\varphi}-\left.\frac{1}{8\left(k_{r} r\right)^{2}}\left(\frac{\partial^{4} p}{\partial \theta^{4}}\right)\right|_{\theta=\varphi}+\cdots\right] .
\end{aligned}
$$

Here, considering the condition $w_{L}(r ; \hat{r})=0$ for $|r-\hat{r}|>L$, the integration range has been formally extended from 0 to $-\infty$ in order to facilitate a comparison with the zero-order Hankel transform.

It can be observed that Eq.(4-15) will again reduce to Eq.(3-30) if we keep only the first term in the bracket and set $\varphi=\theta_{0}$. As a matter of fact, this situation corresponds to the cylindrically symmetric case because $\partial^{2 n} p / \partial \theta^{2 n}=0(n \geq 1)$. Accordingly, the rest of the terms represent the effect of horizontal refraction and can be considered to be small if these derivatives are small. Therefore Eq.(4-15) shows that the general Hankel transform can be represented by a zero-order Hankel transform as a primary term plus additional terms which account for horizontal refraction, as expected in Section 4.1. 


\subsubsection{Analysis of the horizontal refraction effect}

In this section, based on the general Hankel transform with a sliding window that was obtained in Section 4.2.1, we explore an approximate method for detecting the horizontal refraction angle in order to determine local eigenvalues.

First, in order to clarify the role of $\varphi$ in Eq.(4-12), let us replace it with a new angle $\delta$ defined by

$$
\delta \equiv \varphi-\hat{\theta}
$$

If the phase front launched from the source in the direction $\hat{\theta}$ passes through the observation point at the angle $\varphi$, then $\delta$ represents the horizontal refraction angle at this point. On use of this angle, Eq.(4-12) becomes

$$
g\left(k_{r}, \delta ; \hat{r}, \hat{\theta}\right) \sim \frac{e^{i \frac{\pi}{4}}}{\sqrt{2 \pi k_{r}}} \int_{-\infty}^{\infty} d r w_{L}(r ; \hat{r}) \sqrt{r} e^{-i\left(1-\frac{1}{2} \delta^{2}\right) k_{r}} \sum_{n=-\infty}^{\infty} C_{n}(r ; \hat{\theta}) e^{-\frac{i}{2 k_{r}}\left(n-\delta k_{r},\right)^{2}} e^{i n \hat{\theta}}
$$

where the integration range has been extended from 0 to $-\infty$ owing to $w_{L}(r ; \hat{r})$. From this form we can interpret Eq.(4-17) by dividing it into the following two stages:

1. By rescaling $k_{r}$ as $k_{r} \longrightarrow\left(1-\frac{1}{2} \delta^{2}\right) k_{r}$, where $1-\frac{1}{2} \delta^{2}$ is the scaling factor, the integral with respect to $r$ can be considered to represent a zero-order asymptotic Hankel transform with the new wavenumber $\left(1-\frac{1}{2} \delta^{2}\right) k_{r}$. Note that this scaling factor approximately stands for $\cos \delta$ since $|\delta|<<1$.

2. This transform then operates on the range-dependent function $F(r, \delta ; \hat{\theta})$ defined by

$$
F(r, \delta ; \hat{\theta}) \equiv \sum_{n=-\infty}^{\infty} C_{n}(r ; \hat{\theta}) e^{-\frac{i}{2 k r}\left(n-\delta k_{r}\right)^{2}} e^{i n \hat{\theta}}
$$




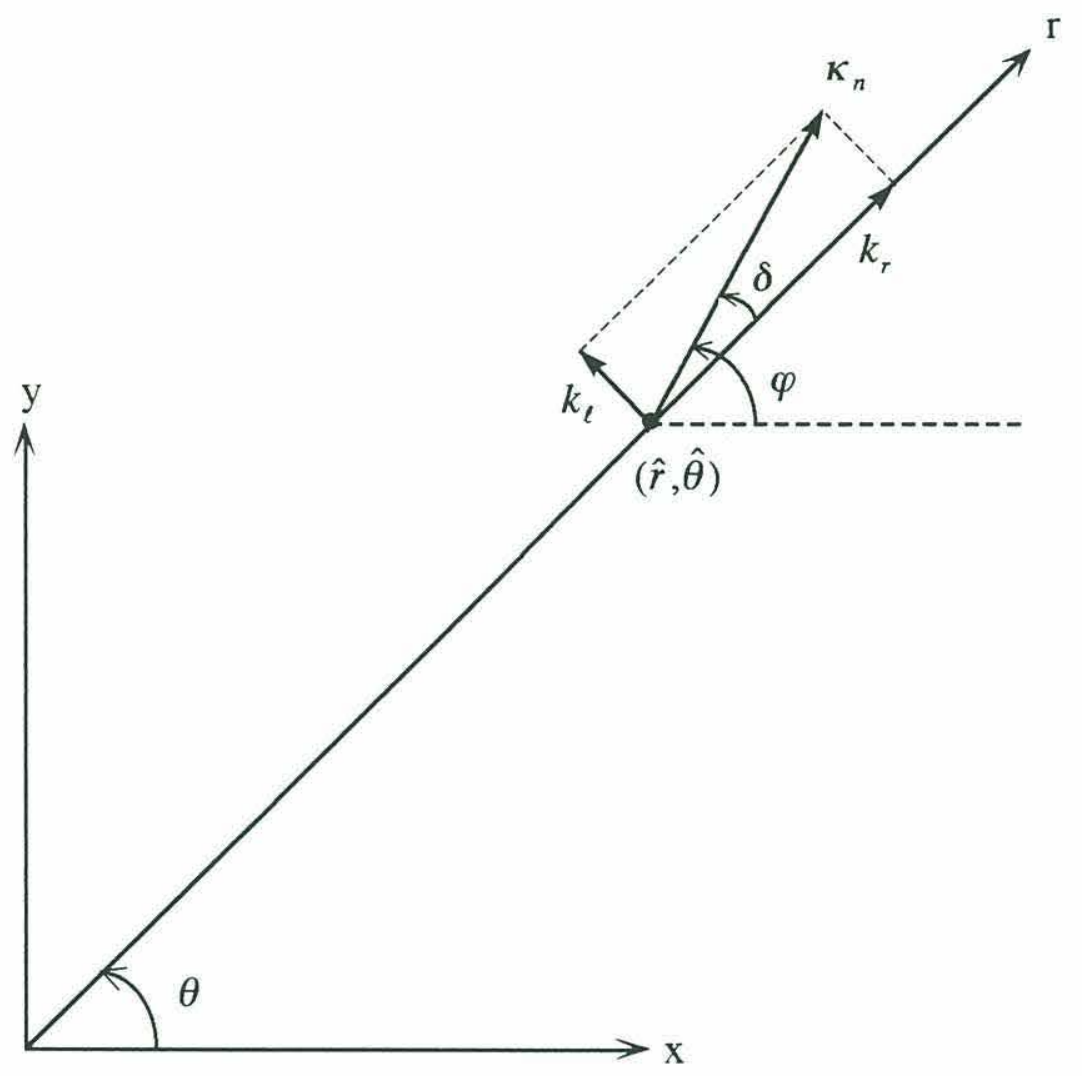

Figure 4-3: Wavenumber and its components projected in the radial and azimuthal directions. 
where $e^{-\frac{i}{2 k_{r} r}(n-\delta k, r)^{2}}$ may be regarded as the weight of the Fourier coefficients in this series. Inside this weighting function, $\delta$ plays the role of a parameter controlling this function.

In order to have more insight into Eq.(4-18), let us express this equation using $k_{\ell}$, i.e., the horizontal wavenumber component perpendicular to $k_{r}$ (see Figure 4-3). As $r$ and $k_{r}$, and as $\theta$ and $n$ are both pairs of conjugate variables, the arc length $\ell$ is also conjugate to $k_{\ell}$. In the Fourier transform, $k_{\ell}$ corresponds to $-i \partial / \partial \ell$ in the same manner as $n$ corresponds to $-i \partial / \partial \theta$. On the other hand, we have the following relation from $\ell=\theta r:$

$$
\frac{1}{r} \frac{\partial}{\partial \theta}=\frac{\partial}{\partial \ell}
$$

Thus, by combining these two relations, it is immediately seen that $k_{\ell}$ and $n$ are related by $k_{\ell}=n / r$. Using this relation, $F(r, \delta ; \hat{\theta})$ can be rewritten in terms of $k_{r}$ and $\ell$, instead of $n$ and $\theta$, as

$$
F(r, \delta ; \hat{\theta}) \equiv \sum_{k_{l}=-\infty}^{\infty} \mathrm{C}_{k_{l}}(r ; \hat{\theta}) e^{-i \frac{k_{r}}{2}\left(\frac{k_{1}}{k_{l}}-\delta\right)^{2}} e^{i k_{\ell} \hat{\ell}},
$$

where $\mathrm{C}_{k_{t}}(r ; \hat{\theta}) \equiv C_{n}(r ; \hat{\theta})$.

Since $k_{r} r \gg 1$ is satisfied, the weighting function $e^{i k_{r} Q}$ with $Q=-\frac{1}{2}\left(k_{\ell} / k_{r}-\delta\right)^{2}$ becomes a rapidly oscillating function except for its stationary points. These points, denoted by $k_{\iota_{s}}$, can be easily determined by solving $[70,73]$

$$
\left.0 \equiv \frac{\partial Q}{\partial k_{\ell}}\right|_{k_{\ell,}}=-\frac{1}{k_{r}}\left(\frac{k_{\ell_{s}}}{k_{r}}-\delta\right) .
$$


Thus $k_{\ell_{s}}$ is related to $\delta$ as

$$
k_{\ell_{s}}=\delta k_{r}
$$

and the primary contribution to $F(r, \delta ; \hat{\theta})$ occurs around these points. Hence, if we fix $\delta$ as $\bar{\delta}$, i.e., fix $\varphi$ as $\hat{\theta}+\bar{\delta}$, in Eq.(4-20), then this weighting function filters out components only around $\bar{k}_{\ell_{s}}\left(=\bar{\delta} k_{r}\right)$, which yields

$$
F(r, \bar{\delta} ; \hat{\theta}) \approx \mathrm{C}_{\bar{k}_{t_{s}}}(r ; \hat{\theta}) e^{i \bar{k}_{t_{s}} \hat{l}},
$$

where $\mathrm{C}_{\bar{k}_{t_{s}}}(r ; \hat{\theta})$ is the Fourier coefficient for $k_{\ell}=\bar{k}_{\ell_{s}}$ (see Figure 4-4). If this component of the given pressure is small, i.e., $C_{\bar{k}_{\ell_{s}}}(r ; \hat{\theta}) \approx 0$, then we have $F(r, \bar{\delta} ; \hat{\theta}) \approx 0$ from Eq.(423) and, consequently, it follows that we have $g\left(k_{r}, \bar{\delta} ; \hat{r}, \hat{\theta}\right) \approx 0$ for this specific $\bar{\delta}$.

The above approximation holds, even when using $n$ instead of $k_{r}$, and so Eq.(4-22) and Eq.(4-23) are rewritten as

$$
n_{s}=\delta k_{r} r
$$

and

$$
F(r, \bar{\delta} ; \hat{\theta}) \approx C_{\bar{n}_{s}}(r ; \hat{\theta}) e^{i \bar{n}_{s} \hat{\theta}},
$$

respectively. We see that, when deriving Eq.(4-25) from Eq.(4-18), $k_{r} r$ is placed in the denominator in the exponential term as compared to being placed in the numerator in Eq.(4-20). But, recalling that $n=k_{\ell} r$, the term $n$ is of order $r$ and consequently the exponential term in Eq.(4-18) turns out to be proportional to $k_{r} r$ in the same way as Eq.(4-20). Thus we can utilize the stationary phase method in using $n$.

When changing the value of $\delta, F(r, \delta ; \hat{\theta})$ varies approximately in accordance with Eq.(4-25) and has its maximums at $\delta_{H}$, where $C_{n}(r ; \hat{\theta})$ is maximum. Physically this 


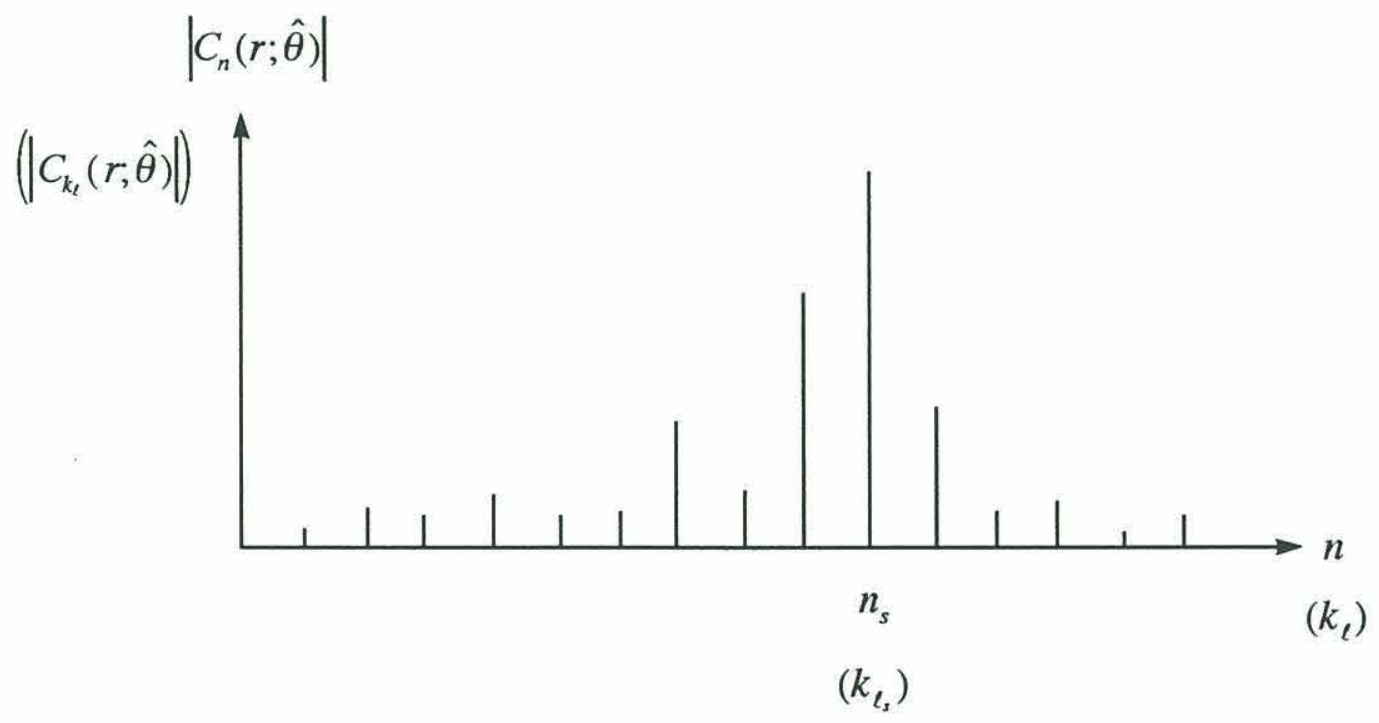

Figure 4-4: Schematic illustration of the Fourier coefficient $\left|C_{n}(r ; \hat{\theta})\right|$, which has its local maximum at $n_{s}$. This can also be represented in terms of $k_{\ell}$ by using $k_{\ell}=r / n$. 
means that each $\delta_{H}$ matches the propagating direction of the major phase front associated with the modes at the observing point $(\hat{r}, \hat{\theta})$. Thus each $\delta_{H}$ represents the horizontal refraction angle of the local modes at $(\hat{r}, \hat{\theta})$. In order to find those $\delta_{H}, F(r, \delta ; \hat{\theta})$ in Eq.(4-18) or $g\left(k_{r}, \delta ; \hat{r}, \hat{\theta}\right)$ in Eq.(4-17) has to be evaluated by changing $\delta$, but it is not straightforward as mentioned in Section 4.2.1.

Instead, let us take the variation of $F(r, \delta ; \hat{\theta})$ with respect to $\delta$. To do this in a simple, approximate manner, one can differentiate $F(r, \delta ; \hat{\theta})$ in Eq.(4-18) with respect to $\delta$ after a Taylor expansion of the weighting function and then keep the leading term:

$$
\frac{\partial F}{\partial \delta} \approx i \sum_{n=-\infty}^{\infty}\left(n-\delta k_{r} r\right) C_{n}(r ; \hat{\theta}) e^{i n \hat{\theta}}
$$

The above $\delta_{H}$ may be approximated then by the angle satisfying $|\partial F / \partial \delta|=0$. In this process, however, $\delta_{H}$ cannot be determined independently of $k_{r}$, since this variable is involved in Eq.(4-26). To represent this condition in terms of $k_{r}$, as well as $\delta$, one can apply the zero-order Hankel transform with the same window $w_{L}$ to Eq.(4-26), after dividing it by $k_{r} r$, yielding

$$
\left|\int_{-\infty}^{\infty} d r w_{L}(r ; \hat{r}) \sqrt{r} e^{-i k, r} \sum_{n=-\infty}^{\infty}\left(\frac{n}{k_{r} r}-\delta\right) C_{n}(r ; \hat{\theta}) e^{i n \hat{\theta}}\right| \approx 0 .
$$

Owing to this approximation, the FFT is now available for use in Eq.(4-27). From this result, we can approximately determine the horizontal refraction angles $\delta_{H}$ in connection with the local eigenvalue or local mode. As presented in Item 1 on page 105 , we changed the scale of the wavenumber by a factor of $1-\frac{1}{2} \delta^{2}$ when operating with the zero-order asymptotic Hankel transform; therefore, the $k_{r}$-scale of its output spectrum has to be multiplied by the reciprocal of this factor. By this process, we can correct the 
underestimated peak position obtained by using the zero-order asymptotic Hankel transform with a sliding window.

In this section, we applied the general Hankel transform with a sliding window to non-cylindrically symmetric fields in an attempt to determine the local eigenvalues in a 3D varying waveguide. By expanding the general Hankel transform in a Taylor series, we found that the first term corresponds to the same form as that obtained for the cylindrically symmetric waveguide in Chapter 3 ; the rest of the terms take on a form given by applying the zero-order Hankel transform with a sliding window to the pressure field differentiated with respect to the azimuthal angle. Therefore, if these terms are small enough to neglect, then the effect of horizontal refraction is negligible. If it is not so, we have to take into consideration the horizontal refraction effect by evaluating the general Hankel transform with a sliding window.

Since we cannot use the FFT when executing the general Hankel transform, we tried to evaluate it analytically in an approximate manner. By resorting to the stationary phase method, we derived an approximate form for this transform. It was then found that the Fourier coefficients play the role of a filter for $\delta$ or $\varphi$ to match the direction of the major phase front. Based on this analysis, we found a method for determining the horizontal refraction angle approximately by taking the first-order variation of this transform with respect to $\varphi$. Finally, by utilizing a scaling factor, $1-\delta^{2} / 2$, we can compensate for the deficit in wavenumber that is obtained when using the zero-order Hankel transform in a 3-D varying waveguide.

In the next section, by exploiting an alternative form of the 2-D Fourier transform, we will examine the effect of horizontal refraction on the zero-order asymptotic Hankel transform with a sliding window when employing it in a non-cylindrically symmetric waveguide. 


\subsection{Study of the horizontal refraction effect using an alternative representation}

In Section 4.2, we studied the effect of horizontal refraction by applying the general Hankel transform with a sliding window to the pressure field in a 3-D varying waveguide. In this section, we will re-examine this effect but will use an alternative representation of a 2-D Fourier transform with the same sliding window in a cylindrical coordinate system. The purpose of this section is to afford a better understanding of the role of the variable $\varphi$ (or $\delta$ ) in the 2-D Fourier transform in connection with the horizontal refraction effect.

To do this, let us first separate the pressure field into its constituent modes with the use of the vertical receiver array fixed at $r=0$ as discussed in Chapter 3.3. The output of the mode filter $q_{n}(r, \theta, z)$ for the $n$th mode can be expressed as

$$
q_{n}(r, \theta, z)=A_{n}(r, \theta, z) e^{i S_{n}(r, \theta)},
$$

where the phase $S_{n}$ represents the accumulated phase along the horizontal ray path $s$ for the $n$th mode on the $\mathrm{x}-\mathrm{y}$ plane:

$$
S_{n}(r, \theta)=\int_{0}^{s(r, \theta)} \kappa_{n} d s
$$

Recalling the relation in Eq.(4-1), we can define the average eigenvalue $K_{n}(r, \theta)$ as

$$
S_{n}(r, \theta) \equiv r K_{n}(r, \theta),
$$

because the range $r$ does not represent the exact integration path length $s$. A Taylor expansion of $K_{n}(r, \theta)$ with respect to the azimuthal angle around $\hat{\theta}$, which is the center direction of the window $v_{\Omega}(\theta ; \hat{\theta})$, yields

$$
K_{n}(r, \theta) \approx \alpha_{n}(r ; \hat{\theta})+\beta_{n}(r ; \hat{\theta})(\theta-\hat{\theta})+\cdots
$$


with

$$
\begin{aligned}
& \alpha_{n}(r ; \hat{\theta}) \equiv K_{n}(r, \hat{\theta})=\frac{1}{r} S_{n}(r, \hat{\theta}), \\
& \left.\beta_{n}(r ; \hat{\theta}) \equiv \frac{\partial K_{n}(r, \theta)}{\partial \theta}\right|_{\hat{\theta}}=\left.\frac{1}{r} \frac{\partial S_{n}(r, \theta)}{\partial \theta}\right|_{\hat{\theta}} .
\end{aligned}
$$

Here let us assume that $\beta_{n}$ can also be obtained approximately by using the phase difference between two output signals of the mode filter:

$$
\beta_{n}(r ; \hat{\theta}) \approx \frac{S_{n}(r, \hat{\theta}+\Delta \theta)-S_{n}(r, \hat{\theta})}{r \Delta \theta},
$$

where the pressure fields corresponding to each signal are separated by $\Delta \theta$ in the azimuthal direction. Thus substitution of Eq.(4-30) and Eq.(4-31) into Eq.(4-28) yields

$$
q_{n}(r, \theta, z) \approx A_{n}(r, \theta, z) e^{i r\left[\alpha_{n}(r)+\beta_{n}(r)(\theta-\hat{\theta})\right]}
$$

Now let us operate on Eq.(4-35) with a 2-D Fourier transform with the same window used in Eq.(4-2):

$$
g\left(k_{r}, \varphi ; \hat{r}, \hat{\theta}\right)=\int_{0}^{\infty} d r w_{L}(r ; \hat{r}) r e^{i r \alpha_{n}(r)} \int_{0}^{2 \pi} d \theta v_{\Omega}(\theta ; \hat{\theta}) A_{n}(r, \theta) e^{i r \beta_{n}(r)(\theta-\hat{\theta})} e^{-i k \operatorname{ros}(\theta-\varphi)},
$$

where the functional notation of $z$ has been omitted for simplicity. For convenience, let us express the phase term inside the $\theta$-integral in Eq.(4-36) as $k_{r} r Q_{n}$ with $Q_{n}(\theta)$ defined by

$$
Q_{n}(\theta, r)=\frac{\beta_{n}(r)}{k_{r}}(\theta-\hat{\theta})-\cos (\theta-\varphi)
$$


Then, owing to $k_{r} r \gg>1$, we can utilize a stationary phase method $[70,73]$ for this integral and determine the stationary point $\theta_{s}$ from the condition satisfying

$$
\left.\frac{\partial Q}{\partial \theta}\right|_{\theta_{s}}=\frac{\beta_{n}(r)}{\kappa_{r}}+\sin \left(\theta_{s}-\varphi\right)=0
$$

By solving this equation for $\theta_{s}$, we have

$$
\theta_{s}=\varphi-\sin ^{-1}\left(\frac{\beta_{n}(r)}{\kappa_{r}}\right)
$$

Use of $\theta$, along with $\frac{\partial^{2} Q}{\partial \theta^{2}}=1$ in the formula of the stationary phase method given by Eq.(3-53) yields

$$
\begin{aligned}
g\left(k_{r}, \varphi ; \hat{r}, \hat{\theta}\right) \sim e^{i \frac{\pi}{4}} \sqrt{\frac{2 \pi}{k_{r} r}} \int_{-\infty}^{\infty} w_{L}(r ; \hat{r}) v_{\Omega}\left(\theta_{s} ; \hat{\theta}\right) A_{n}\left(r, \theta_{s}\right) \\
\\
\quad \times e^{i\left[\left[\alpha_{n}(r)+\beta_{n}(r)\left(\varphi-\theta_{0}\right)-\beta_{n}(r) \sin ^{-1}\left(\frac{\beta_{n}(r)}{k_{r}}\right)-k_{,} \cos \left(\sin ^{-1}\left(\frac{\beta_{n}(r)}{k_{k}}\right)\right)\right]\right.} r d r \quad,
\end{aligned}
$$

Since $\left|\beta_{n}(r)\right|<<k_{r}$, due to the assumption of gradual variation of the medium, we may use the following approximation in Eq.(4-40):

$$
\sin ^{-1}\left(\frac{\beta_{n}(r)}{\kappa_{r}}\right) \approx \frac{\beta_{n}(r)}{\kappa_{r}}
$$

and

$$
\cos \left(\sin ^{-1}\left(\frac{\beta_{n}(r)}{\kappa_{r}}\right)\right) \approx 1-\frac{1}{2}\left(\frac{\beta_{n}(r)}{\kappa_{r}}\right)^{2}
$$

As a result, Eq.(4-40) becomes 


$$
g\left(k_{r}, \varphi ; \hat{r}, \hat{\theta}\right) \sim e^{i \frac{\pi}{4}} \sqrt{\frac{2 \pi}{k_{r}}} v_{\Omega}(\varphi ; \hat{\theta}) \int_{-\infty}^{\infty} w_{L}(r ; \hat{r}) A_{n}(r, \varphi) e^{i\left[\alpha_{n}(r)+\beta_{n}(r)(\varphi-\hat{\theta})-\frac{\beta_{n}^{2}(r)}{2 k_{r}}-k_{r}\right]} \sqrt{r} d r \quad,
$$

where we changed the integration range in the same way as in Section 4.2.2 and approximated the amplitude term as $v_{\Omega}\left(\theta_{s} ; \hat{\theta}\right) \approx v_{\Omega}(\varphi ; \hat{\theta})$ and $A_{n}\left(r, \theta_{s}\right) \approx A_{n}(r, \varphi)$ by dropping $\beta_{n}(r) / k_{r}$ in Eq.(4-39) because of its negligible effect. But we cannot neglect it in the phase term. As discussed in the previous section, if we can match $\varphi$ to the propagation direction of the phase front associated with the $n$th mode at the observing point $(\hat{r}, \hat{\theta})$, then the exact wavenumber for the local eigenvalue may be obtained from Eq.(4-43).

Therefore, let us study the effect of horizontal refraction by specifying the values of $\varphi$ in the form of a 2-D Fourier transform given by Eq.(4-43).

1. Case of $\varphi=\hat{\theta}$

By setting $\varphi=\hat{\theta}$ in Eq.(4-43), we have

$$
g\left(k_{r}, \hat{\theta} ; \hat{r}, \hat{\theta}\right) \sim \frac{e^{i \frac{\pi}{4}}}{\sqrt{2 \pi k_{r}}} \int_{-\infty}^{\infty} w_{L}(r ; \hat{r}) A_{n}(r, \hat{\theta}) e^{i\left[\alpha_{n}(r)-\frac{\beta_{n}^{2}(r)}{2 k_{r}}-k_{r}\right]} \sqrt{r} d r
$$

or, using the definition $q_{n}(r, \hat{\theta})=A_{n}(r, \hat{\theta}) e^{i r \alpha_{n}(r)}$,

$$
g\left(k_{r}, \hat{\theta} ; \hat{r}, \hat{\theta}\right) \sim \frac{e^{i \frac{\pi}{4}}}{\sqrt{2 \pi k_{r}}} \int_{-\infty}^{\infty} w_{L}(r ; \hat{r}) q_{n}(r, \hat{\theta}) e^{-i r \frac{\beta_{n}^{2}(r)}{2 k_{r}}} e^{-i k_{r} r} \sqrt{r} d r
$$

where $v_{\Omega}(\hat{\theta}, \hat{\theta})=1$ has been employed. It is observed that Eq.(4-45) differs from the zero-order asymptotic Hankel transform in Eq.(3-30), which was obtained for the cylindrically symmetric case, by a factor of $e^{-i \frac{\beta_{s}^{2}(r)}{2 k_{r}}}$. If the sign of the exponential term is positive, then we can obtain the peak position close to the local eigenvalue as will be 
detailed in Item 3. But, in this case, we obtain an underestimate value from the peak position in the output spectrum of Eq.(4-45) as explained in Item 3 again.

2. Case of $\varphi=\hat{\theta}+\beta_{n}(r) / 2 k_{r}$

If we set

$$
\varphi=\hat{\theta}+\frac{\beta_{n}(r)}{2 k_{r}},
$$

then it follows from Eq.(4-43) that

$$
g\left(k_{r}, \hat{\theta}+\frac{\beta}{2 k_{r}} ; \hat{r}, \hat{\theta}\right) \sim \frac{e^{i \frac{\pi}{4}}}{\sqrt{2 \pi k_{r}}} \int_{-\infty}^{\infty} w_{L}(r ; \hat{r}) A_{n}(r, \hat{\theta}) e^{i\left[\alpha_{n}(r)-k_{r}\right]} \sqrt{r} d r
$$

where $\beta_{n} / 2 k_{r}$ in the argument for $v_{\Omega}$ and $A_{n}$ has been neglected in the same manner as in deriving Eq.(4-43). If we rewrite Eq.(4-47) by using $q_{n}(r, \hat{\theta})$, it follows that

$$
g\left(k_{r}, \hat{\theta}+\frac{\beta_{n}}{2 k_{r}} ; \hat{r}, \hat{\theta}\right) \sim \frac{e^{i \frac{\pi}{4}}}{\sqrt{2 \pi k_{r}}} \int_{-\infty} w_{L}(r ; \hat{r}) q_{n}(r, \hat{\theta}) e^{-i k r} \sqrt{r} d r
$$

Here we notice that the right-hand side of Eq.(4-48) is equivalent to the zero-order asymptotic Hankel transform with the sliding window in Eq.(3-30), which was obtained in the cylindrically symmetric waveguide. As stated in Section 4.2, the peak position determined from this spectrum is always underestimated due to the horizontal refraction effect.

3. Case of $\varphi=\hat{\theta}+\beta_{n}(r) / k_{r}$

By noticing that $\beta_{n}$ corresponds to $k_{\ell}(=n / r)$, the wavenumber component in the azimuthal direction defined in Section 4.2, we can predict the refraction angle $\delta \equiv \varphi-\hat{\theta}$ from Eq.(4-27) as: 


$$
\varphi=\hat{\theta}+\frac{\beta_{n}(r)}{k_{r}}
$$

This relation can also be understood from a physical point of view if $k_{r} \approx \kappa_{n}$. As illustrated in Figures 4-1 and 4-3, if the phase front associated with the $n$th mode passes through the observation point $(\hat{r}, \hat{\theta})$, then $\delta \equiv \varphi-\hat{\theta}$ is related to the wavenumbers $k_{r}$ and $\beta_{n}$ as $\beta_{n} / k_{r} \approx \tan \delta \approx \delta$. Here the horizontal refraction angle $\delta$ is considered relatively small. Thus employment of this relation in Eq.(4-43) yields

$$
g\left(k_{r}, \hat{\theta}+\frac{\beta_{n}}{k_{r}} ; \hat{r}, \hat{\theta}\right) \sim \frac{e^{i \frac{\pi}{4}}}{\sqrt{2 \pi k_{r}}} \int_{--}^{-} w_{L}(r ; \hat{r}) A_{n}(r, \hat{\theta}) e^{i\left[\alpha_{n}(r)+\frac{\beta_{n}^{2}(r)}{2 k_{r}}-k_{r}\right]} \sqrt{r} d r
$$

or, equivalently by using $q_{n}(r, \hat{\theta})=A_{n}(r, \hat{\theta}) e^{i r \alpha_{n}(r)}$,

$$
g\left(k_{r}, \hat{\theta}+\frac{\beta_{n}}{k_{r}} ; \hat{r}, \hat{\theta}\right) \sim \frac{e^{i \frac{\pi}{4}}}{\sqrt{2 \pi k_{r}}} \int_{-.}^{-} w_{L}(r ; \hat{r}) q_{n}(r, \hat{\theta}) e^{i \frac{\beta_{n}^{2}(r)}{2 k_{r}}} e^{-i k, r} \sqrt{r} d r
$$

As pointed out in Section 4.2.1, we cannot evaluate Eq.(4-51) using the FFT due to the

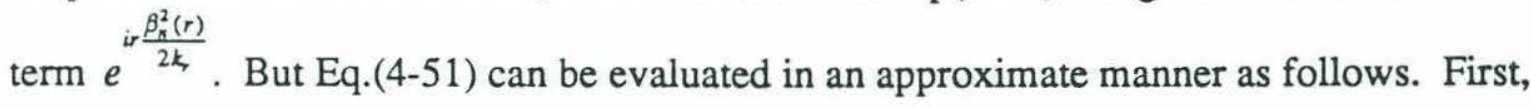
let Eq.(4-51) be rewritten as

$$
g\left(k_{r}, \hat{\theta}+\frac{\beta_{n}}{k_{r}} ; \hat{r}, \hat{\theta}\right) \sim \frac{e^{i \frac{\pi}{4}}}{\sqrt{2 \pi k_{r}}} \int_{-\ldots} w_{L}(r ; \hat{r}) q_{n}(r, \hat{\theta}) e^{-i\left(1-\frac{1}{2}\left(\frac{\beta_{n}(r)}{k_{r}}\right)^{2}\right) k_{k}} \sqrt{r} d r
$$

If we replace $\beta_{n} / k_{r}$ with $\delta$ in Eq.(4-52), then the resulting form can be identified with the integral with respect to $r$ in Eq.(4-17). Thus, following the description in Item 1, page 105, we can consider Eq.(4-52) as a zero-order Hankel transform with a sliding window 
by changing the scale of $k_{r}$ by a factor of $1-\frac{1}{2} \delta^{2}$. Since $\delta$ is a function of $k_{r}$, the above statement is not completely correct; but, we can fix $k_{r}$ in $\delta\left(=\beta_{n} / k_{r}\right)$ by giving some approximate value $\left(\bar{\kappa}_{n}(r)\right)$ that is close to the exact local eigenvalue $\kappa_{n}(r)$. For example, we can use the peak position in the output spectrum of Eq.(4-48) for $\bar{\kappa}_{n}(r)$. Of course, this is not an exact local eigenvalue, but $\kappa_{n}(r)-\bar{\kappa}_{n}(r)$ is of second order, so this choice does not affect $1-\frac{1}{2} \delta^{2}$ much to leading order. More simply, if we fix $\beta_{n}(r)$ in Eq.(4-52) as $\beta_{n}(\hat{r})$, then the scale factor above can be immediately calculated and the local eigenvalue at the observation point $\hat{r}$ is approximately given by

$$
\kappa_{n}(\hat{r}) \approx \bar{\kappa}_{n}(\hat{r})+\frac{\beta_{n}^{2}(\hat{r})}{2 \bar{\kappa}_{n}(\hat{r})}
$$

Thus the above procedure can be summarized as follows: (1) First we apply the zeroorder asymptotic Hankel transform with a sliding window to the output of the mode filter even in a horizontally refracting environment; and (2) We compensate for the underestimated output by using $\beta_{n}(r)$ as shown in Eq.(4-53) to obtain the local eigenvalues.

Of particular interest is that, as seen from a comparison of Eq.(4-46) and Eq.(4-49), the zero-order asymptotic Hankel transform in Eq.(4-48) corresponds to the 2-D transform obtained when adjusting $\varphi$ by one-half of the deflection angle, i.e., $\beta_{n} / 2 \kappa_{n}$. The deficit in adjustment for $\varphi$ results in an underestimate for the local eigenvalue as shown in Eq.(4-53).

As noted in Item 1 (page 115), i.e., the case of $\varphi=\hat{\theta}$, the underestimated amount becomes $\beta_{n}^{2} / \kappa_{n}$ because of the factor $e^{i r \frac{\beta_{n}^{2}(r)}{2 k_{n}}}$, which differs from $e^{-i r \frac{\beta_{n}^{2}(r)}{2 k_{r}}}$ by $\beta_{n}^{2} / \kappa_{n}$ in the exponent. So this factor serves to increase the deficit in the peak position in the output spectrum. 
In the next section, the result in Eq.(4-53) will be compared numerically to the results obtained using the general Hankel transform in Section 4.2. The method used in this section would not be practically applicable to real data, because we cannot expect to obtain the stable phase difference $\beta_{n}(r)$ due to the effect of noise. In this section, we focused rather on the analytical description of horizontal refraction by deriving an alternative representation with the use of the phase difference $\beta_{n}(r)$ in the different azimuthal angles. We also compared the resulting transform to the zero-order asymptotic Hankel transform with a sliding window and found that the underestimated amount for the local eigenvalue in the latter transform can be expressed in terms of the phase difference $\beta_{n}(r)$.

\subsection{Analysis of simulated data}

In this section, we will examine numerically the methods obtained from the previous sections to determine the local eigenvalues in a 3-D varying waveguide. Here we will utilize a non-cylindrically symmetric pressure field which is simulated by using the horizontal ray method.

\subsubsection{Bottom environment and field simulation}

We will use a wedge-type bottom as shown in Figure 4-5. The range-dependent parameters characterizing the bottom sediment are given as a function of the $x$-coordinate

only, so that the ocean environment does not change in the $y$-direction. As for the bottom change in the $x$-direction, we set the same variation used for the bottom model in Chapter 3.4. Hence Figures 3-9 through 3-14 hold true for the current bottom model by replacing the variable $r$ with $x$. If we employ the same source whose frequency is $75 \mathrm{~Hz}$, then we have the equivalent local modes at $r=0$ as shown in Figures 3-15 and 3-16. In this ocean environment, the local modes also depend on the range $x$ only and remain unchanged in the $y$-direction. Thus the local eigenvalues $\kappa_{n}(x)$ take on the same values as those in 


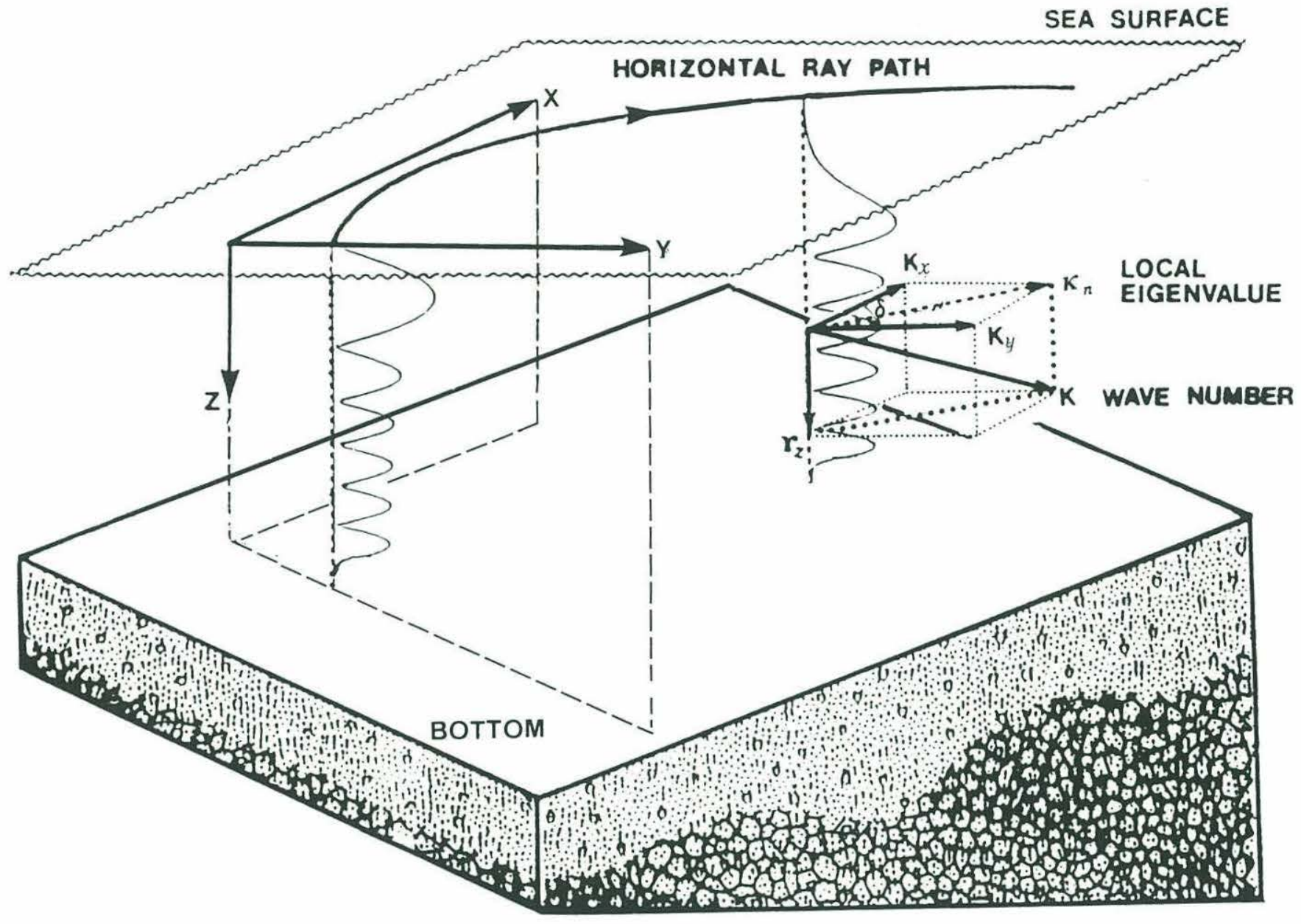

Figure 4-5: Geometry of the ocean model. 
Figure 3-18. The pressure field, however, is different from that in Chapter 3.4 due to the effect of horizontal refraction, as explained in Chapter 2. To accommodate this effect in the field simulation, we can use the horizontal ray theory that was described in Chapter 2.4.

Since $\kappa_{n}(x)$ has already been given along the $x$-axis by solving the depth equation for each local mode, the horizontal ray path associated with this mode can be easily determined by applying $\kappa_{n}(x)$ to the ray equation defined by Eq.(2-16). Then we can calculate the phase by integrating $\kappa_{n}(x)$ along this ray path and thus obtain the pressure field by using Eq.(2-17).

Figure 4-6 represents the contour map for the horizontal refraction angle $\delta_{n}$ of the third mode with respect to the range $r$ and azimuthal angle $\theta$ of the observation point. Here we chose only to show the third mode because it is representative of the other modes. From this figure it can be seen that horizontal refraction takes place most strongly around $\theta=70^{\circ}$ from the $x$-axis. When we fix the azimuthal angle to be $70^{\circ}$, Figure 4-7 shows the refraction angle $\delta_{n}$ for different modes. As seen from this result, in general, the higher the mode, the stronger the horizontal refraction. The angle of horizontal refraction is one of the unknown variables that we have to infer by applying the general Hankel transform with a sliding window to the pressure field.

Before proceeding, it would be of interest to compare the field obtained by the horizontal ray method with the field given by the Nx2D method, because the difference in the results of these two methods is due to the horizontal refraction as discussed in Chapter 2. Figure 4-8 compares these two fields, which are simulated using the first five modes, in the azimuthal direction of $70^{\circ}$. The overlap of the two curves demonstrates that the pressure field is almost equivalent, and thus the Nx2D method can provide a good approximation, as far as the amplitude of the pressure field is concerned. In the present example no substantial difference occurs because the pressure field is dominated by the 


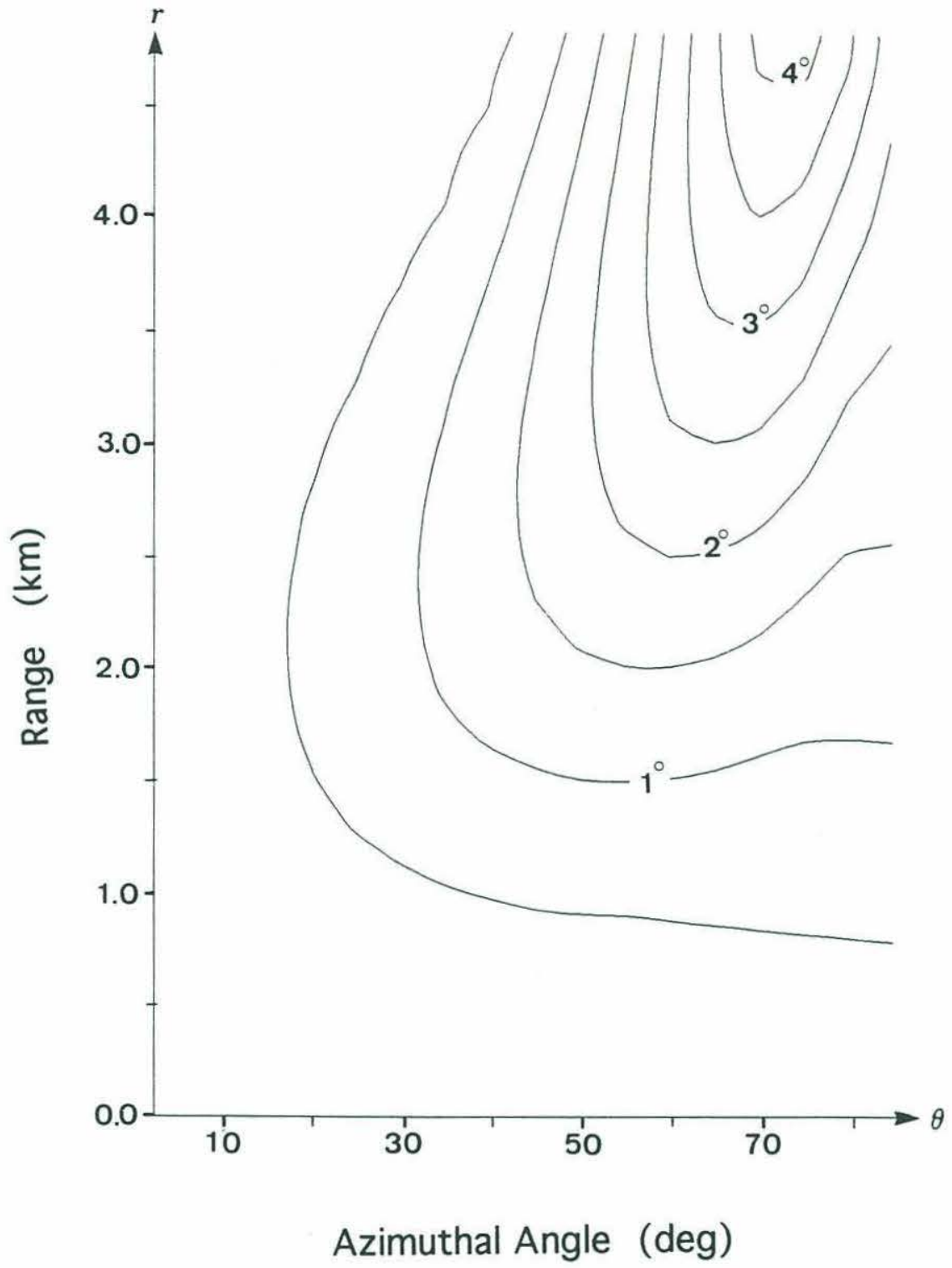

Figure 4-6: Contour map for the horizontal refraction angle of the third mode. 


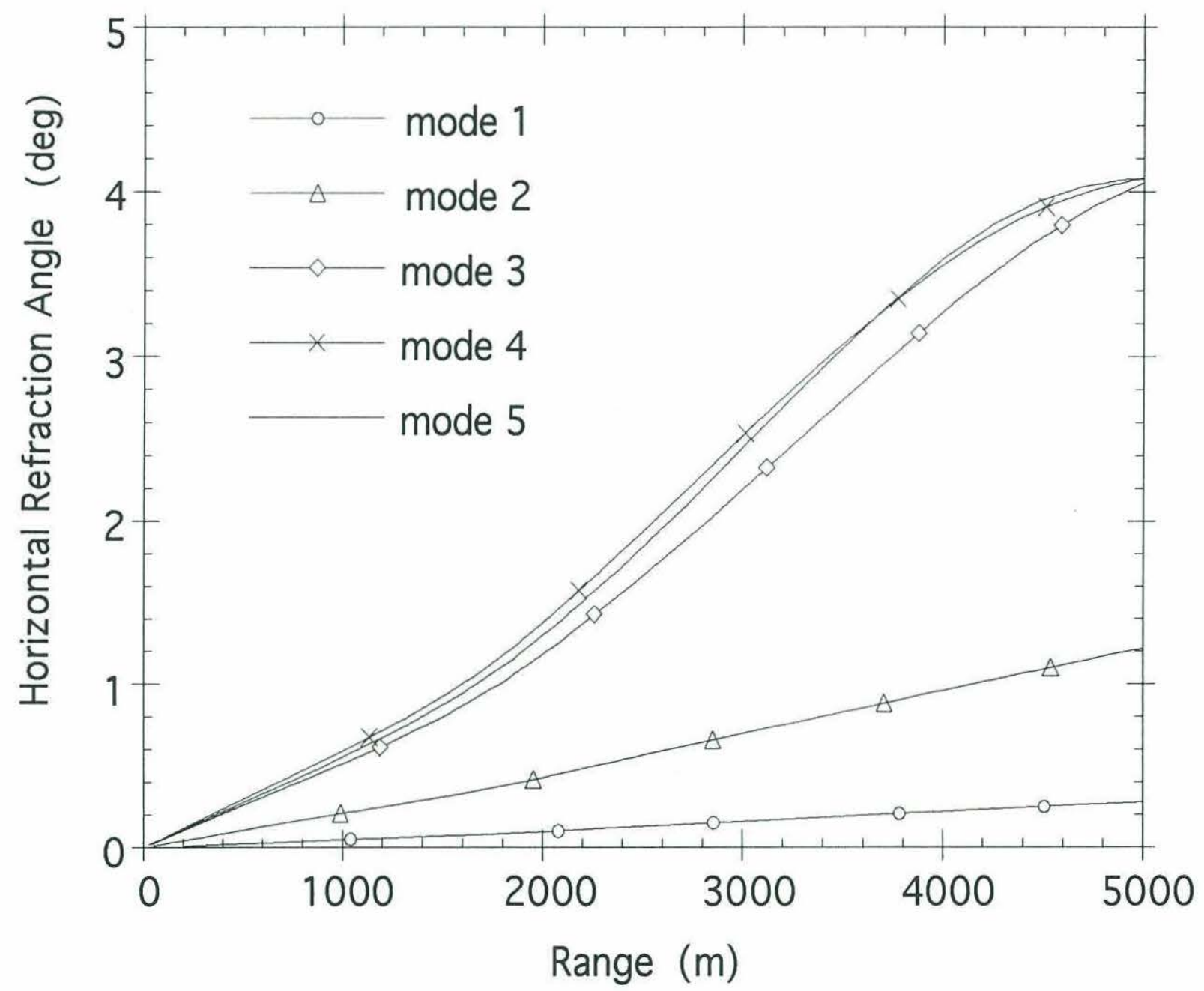

Figure 4-7: Comparison of horizontal refraction angles for different modes when setting the azimuthal direction at $70^{\circ}$. 


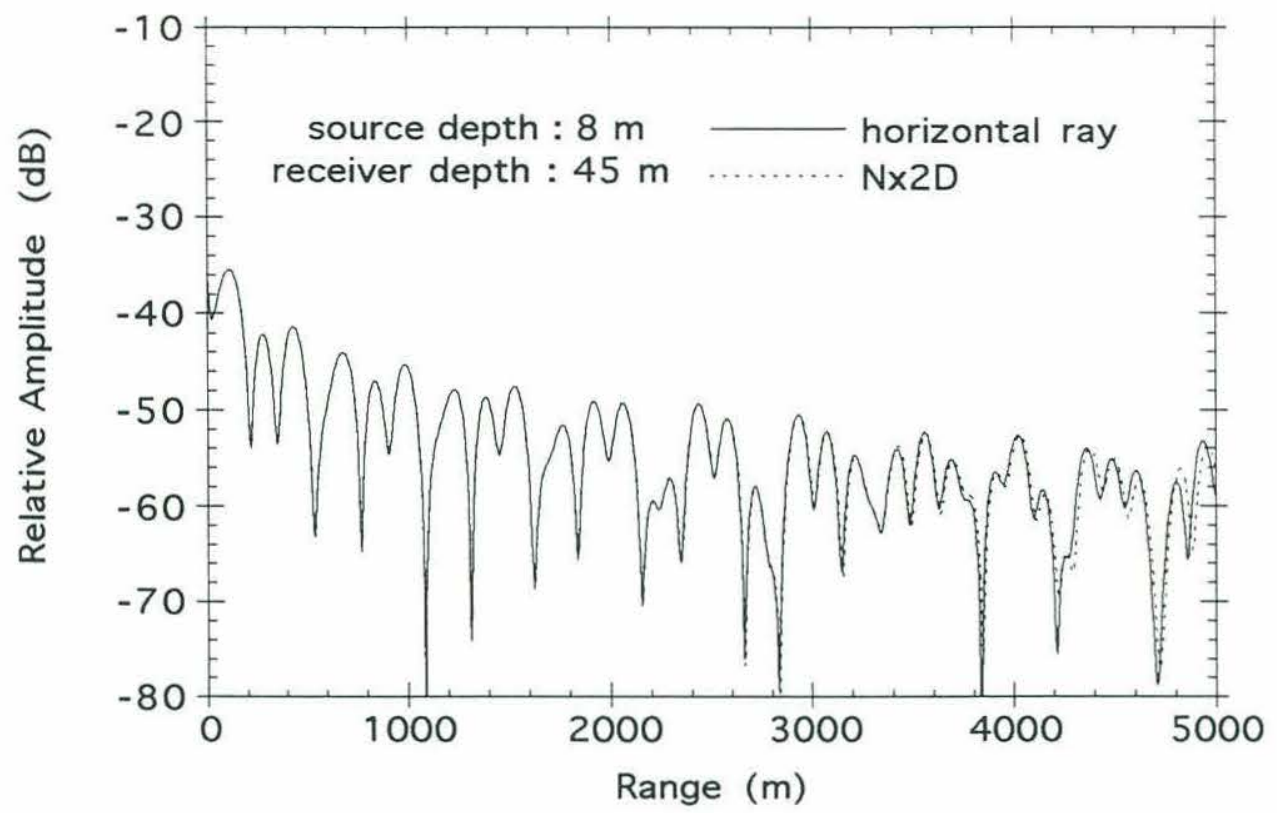

Figure 4-8: Comparison of the pressure fields simulated by the horizontal ray method and the NX2D method when setting the azimuthal direction at $70^{\circ}$.

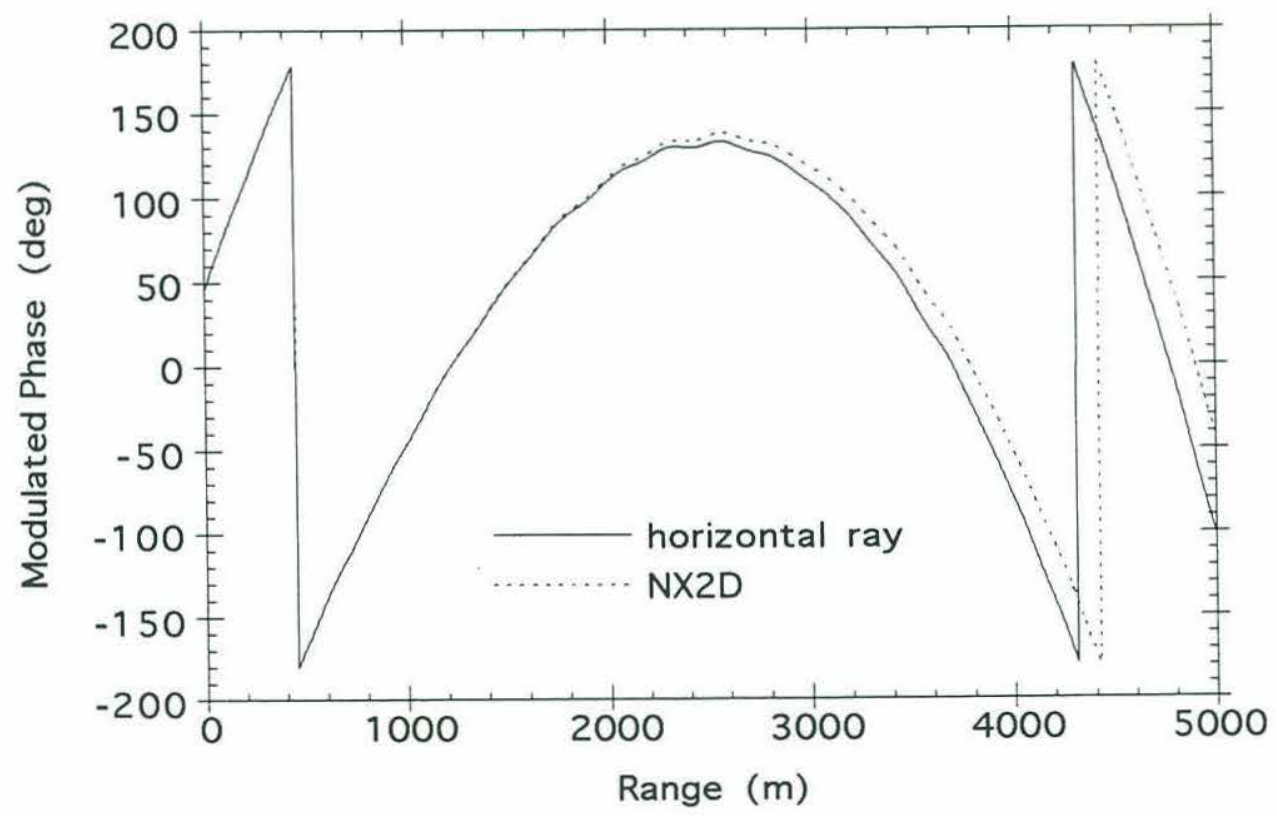

Figure 4-9: Comparison of the phases of the third mode simulated by the horizontal ray method and the NX2D method when setting the azimuthal angle at $\theta=70^{\circ}$. Both phases are modulated as $\Phi(r, \theta) \rightarrow \Phi(r, \theta)-k_{0} r$ with $k_{0}=\omega / c_{0}$ and $c_{0}=1660 \mathrm{~m} / \mathrm{s}$. 
lowest two modes, which have relatively weak horizontal refraction as seen from Figure 4-8.

If we choose the third mode alone and compare its phase as obtained from the horizontal ray method and the Nx2D method, then the difference in these two phases can be clearly observed for ranges over $3000 \mathrm{~m}$, as shown in Figure 4-9. Note that both phases $\left.\Phi(r, \theta)\right|_{\theta=70^{.}}$are modulated as $\Phi(r, \theta) \rightarrow \Phi(r, \theta)-k_{0} r$ with $k_{0}=\omega / c_{0}$ and $c_{0}=1660 \mathrm{~m} / \mathrm{s}$, in order to effect the comparison. As discussed in Section 4.1, the difference in the phase, or more specifically, the spatial frequency (horizontal wavenumber), plays an important role in describing the horizontal refraction accurately for a particular mode.

\subsubsection{Application of the general Hankel transform with a sliding window}

Now, we will try to determine the local eigenvalues in this horizontally refracting environment by applying the general Hankel transform with a sliding window to the noncylindrically symmetric field simulated above.

In the first stage, let us assume that, due to measurement limitations, the pressure field $p(r, \theta)$ is given only in the radial direction at selected azimuthal angles, i.e., azimuthally every $\Delta \theta$ (see Figure 4-20(a)). Thus, when calculating the Fourier coefficient $C_{n}(r ; \hat{\theta})$ in Eq.(4-6) using the FFT, the sampling interval in azimuthal angle is automatically limited to $\Delta \theta$. Consequently, the sample distance $r \Delta \theta$ in the azimuthal direction increases with increasing range $r$, and at some range violates the spatial Nyquist criterion [80]. As a result of aliasing errors, the general Hankel transform based on $C_{n}$ does not work properly beyond this range. Therefore, for this transform to perform correctly, there must exist a maximum range, which depends on $\Delta \theta$ and also the degree of horizontal refraction of the field. This problem will be discussed in the next section. 
Since horizontal refraction occurs most strongly in an azimuthal direction around $70^{\circ}$, as seen from Figure 4-6, we will examine the performance of the general Hankel transform by setting the center of the azimuthal window in this direction.

Before doing this, let us show that we have a deficit in the peak position of the output spectrum due to the effect of horizontal refraction if the zero-order Hankel transform with a sliding window is applied to the pressure field in this direction. Figure 4-10 compares the local eigenvalue of the third mode and the peak position obtained by using the Hanning window with $L=1000 \mathrm{~m}$ that is slid every $50 \mathrm{~m}$. Here the pressure field for the third mode has been separated by mode filtering with the use of a vertical array of receivers that is placed at $r=0$ as shown in Figure 3-23. We observe that the deficit in the horizontal wavenumber increases in accordance with the increase of the horizontal refraction angle. To confirm this, the deficit is plotted in Figure 4-11 and is compared with $\kappa_{n}-\kappa_{n} \cos \delta_{n}$, where $\delta_{n}$ is the horizontal refraction angle shown in Figure 4-7.

As discussed in Section 4.1, what is required in the first place is to determine the horizontal refraction angle $\delta_{n}$ for each mode at various ranges. When setting the center of the window as $\hat{\theta}=70^{\circ}$ and $\hat{r}=4250 \mathrm{~m}$, the window length as $\Omega=10^{\circ}$ and $L=1000 m$, and $\Delta \theta=1^{\circ}$, Figure 4-12(a) shows the value of the left-hand side in Eq.(427) with respect to both the horizontal wavenumber $k_{r}$ and the refraction angle $\delta$. As long as the ridge corresponding to each mode is separated far enough apart to avoid interference from each other, the propagation direction of the phase front associated with each mode can then be determined from the angle $\delta_{H}$ at which the value in Eq.(4-27) takes a local minimum. In Figure 4-12(a), we can find $\delta_{H}$ for the first and second modes but it is hard to locate the angle $\delta_{H}$ for the third through fifth modes due to interference. In particular, the ridge associated with the fourth mode is completely buried in the tail of adjacent modes and cannot be recognized. In order to improve this situation, we can increase the window length $L$. Figure 4-12(b) shows the result for $L=1500 \mathrm{~m}$. 


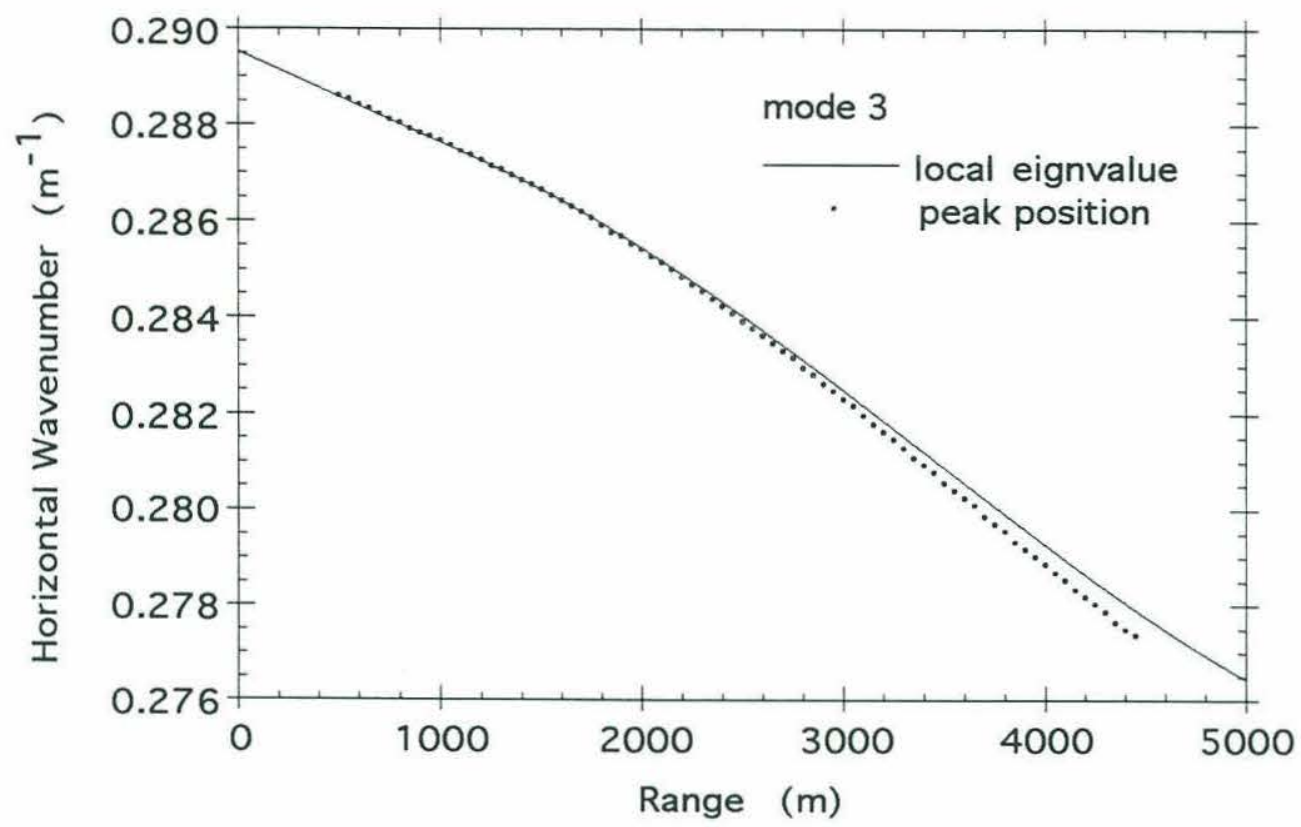

Figure 4-10: Comparison of local eigenvalue and peak position that is obtained by applying the zero-order Hankel transform with the sliding Hanning window with length $L=1000 \mathrm{~m}$ to the pressure field in the azimuthal direction $\theta=70^{\circ}$. The pressure field for the third mode has been separated by mode filtering with the use of the vertical array in Figure 3-23.

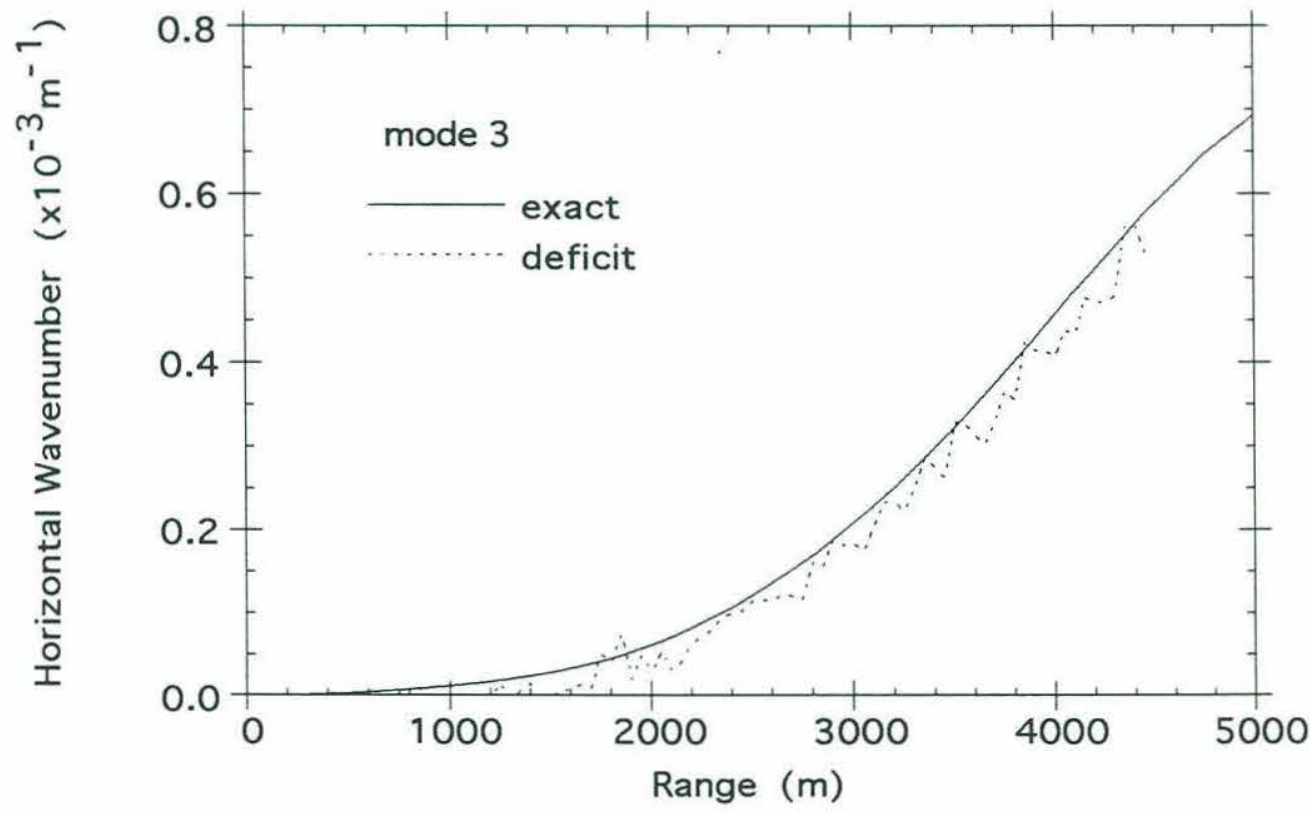

Figure 4-11: Comparison of the deficit in the wavenumber in Figure 4-10 and the exact value $\left(\kappa_{n}-\kappa_{n} \cos \delta_{n}\right)$. 


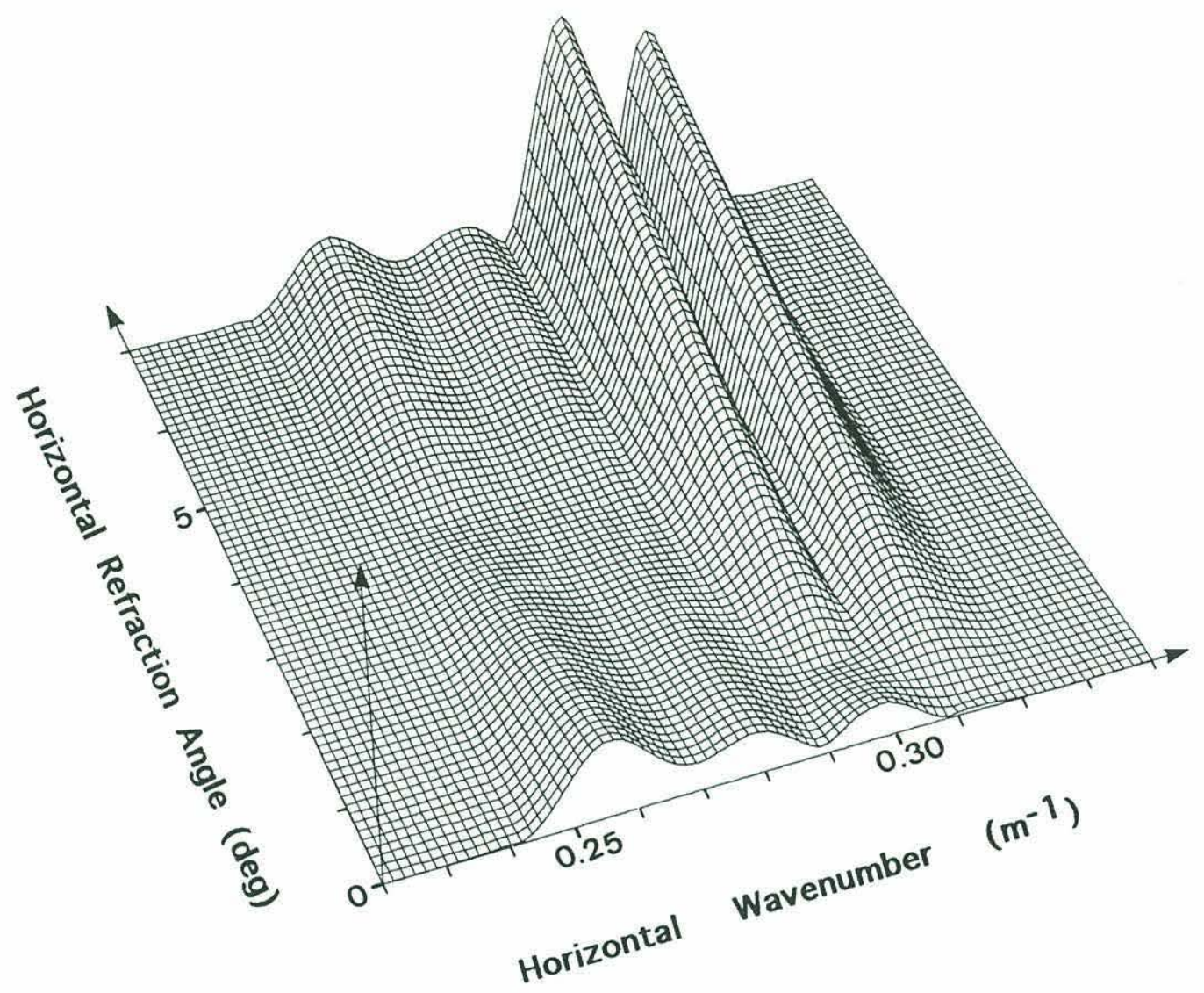

(a)

Figure 4-12: Left-hand side versus $k_{r}$ and $\delta$ of Eq.(4-27) when setting the center of the Hanning window as $(\hat{r}, \hat{\theta})=\left(4250 \mathrm{~m}, 70^{\circ}\right)$, the width of window as $\Omega=10^{\circ}$, and the sampling width as $\Delta \theta=1^{\circ}$ : (a) window length $L=1000 \mathrm{~m}$ and (b) window length $L=1500 \mathrm{~m}$. 


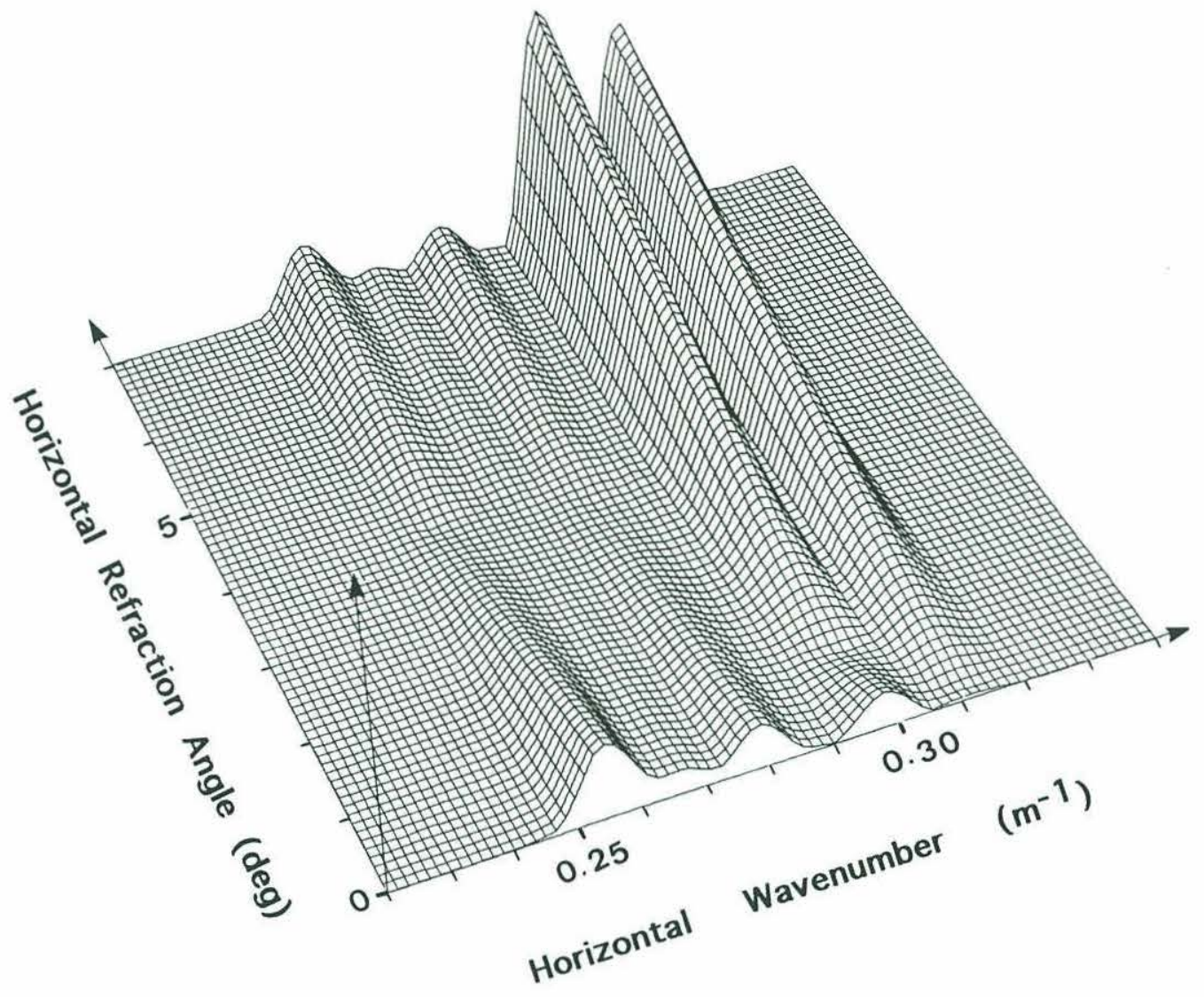

(b)

Figure 4-12, continued. 
Although we could improve the precision of the angle $\delta_{H}$ for the third and fifth modes, $\delta_{H}$ for the fourth mode is still hard to localize.

Thus, as we had shown previously in Chapter 3, we need to separate the pressure field by modes before applying the above transform. By incorporating data from the vertical array shown in Figure 3-23, we can perform mode filtering. Figures 4-13(a) and 4-13(b) show the results for the second and third modes, respectively, when applying the transform with $L=1000 \mathrm{~m}$ after the separation of modes; these two modes are representative of ones having relatively strong and weak refraction, respectively, as shown in Figure 4-7. As seen from the results in Figure 4-13(a) and 4-13(b), we can easily find $\delta_{H}$ in the single mode situation.

Here it would be of interest to examine the value of the Fourier coefficient $C_{n}(r ; \hat{\theta})$. In accordance with Eq.(4-24) and Eq.(4-25), as discussed in Section 4.2.3, this coefficient takes on its maximum value at $\delta_{H}$ found above, where the spectrum of the general Hankel transform takes its maximum. Figure 4-14 shows the absolute value of $C_{n}(r ; \hat{\theta})$ at the same range $(r=4250 \mathrm{~m})$ after the separation of modes. In this figure, the coefficients at the other ranges are also compared, where each $\left|C_{n}(r ; \hat{\theta})\right|$ is normalized so that its maximum value becomes one. In the same manner, Figure 4-15 shows the range evolution of $\left|C_{n}(r ; \hat{\theta})\right|$ in the azimuthal direction $\hat{\theta}=70^{\circ}$. It can be observed that $n_{s}$, at which $\left|C_{n}(r ; \hat{\theta})\right|$ takes on its maximum value, varies with range.

Let us next examine the maximum range for the general Hankel transform to work properly when changing the sampling distance $\Delta \theta$ in the azimuthal angle. Figure 4-16 shows this result for the different $\Delta \theta$ and also compares with the exact refraction angle of the horizontal ray $\left(\delta_{n}\right)$. As discussed in the beginning of this subsection, the maximum range for this transform to hold true decreases with increasing $\Delta \theta$. From the spatial Nyquist rate that is given by $\pi / \kappa_{n} \sin \delta_{H}$, we can see that the results in Figure 4-16 match roughly this condition. 


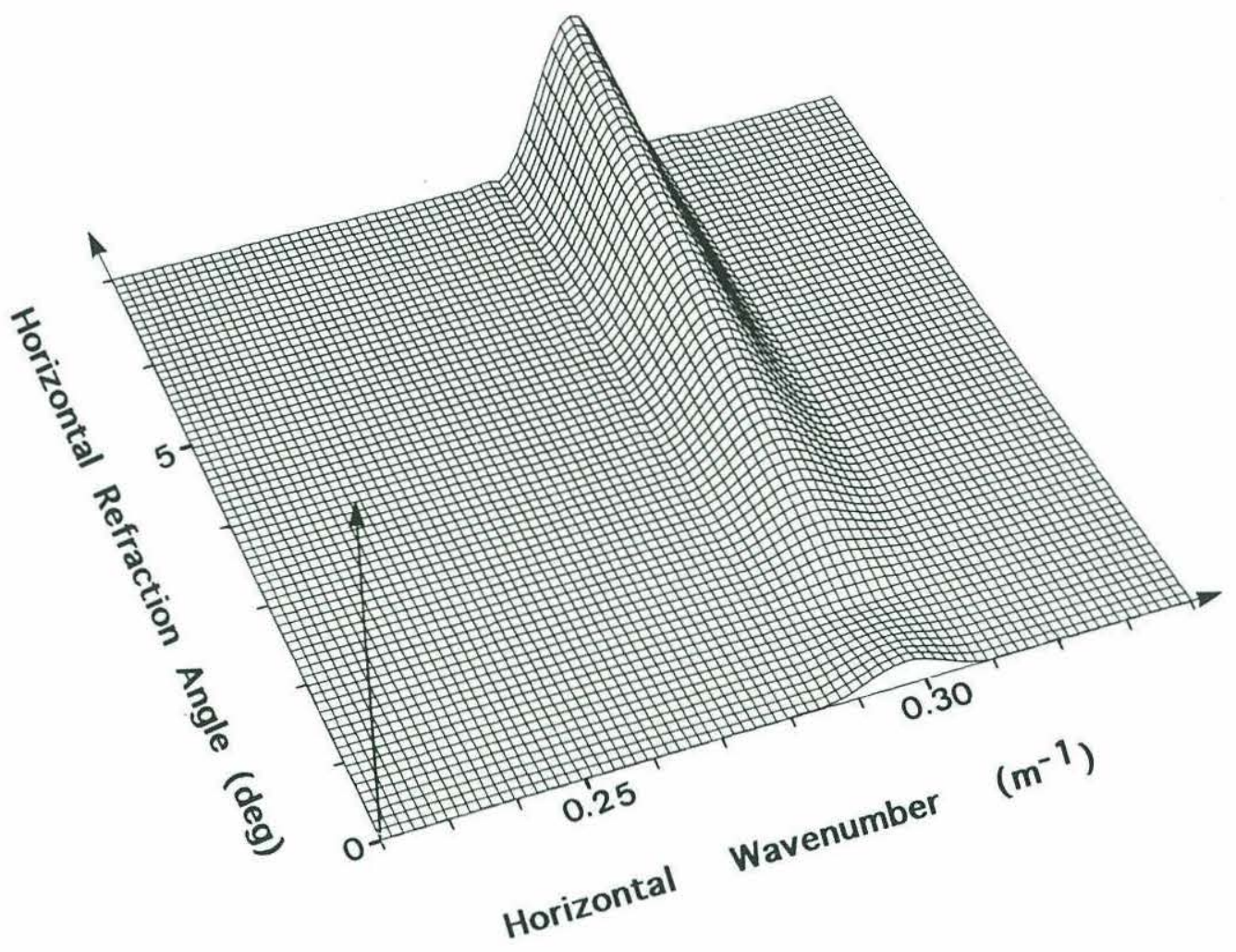

(a)

Figure 4-13: Left-hand side of Eq.(4-27) versus $k_{r}$ and $\delta$ after separation of modes when setting the center of the Hanning window as $(\hat{r}, \hat{\theta})=\left(4250 \mathrm{~m}, 70^{\circ}\right)$, the window length as $(L, \Omega)=\left(1000 \mathrm{~m}, 10^{\circ}\right)$, and the sampling width as $\Delta \theta=1^{\circ}$ : (a) mode 2 and (b) mode 3. 


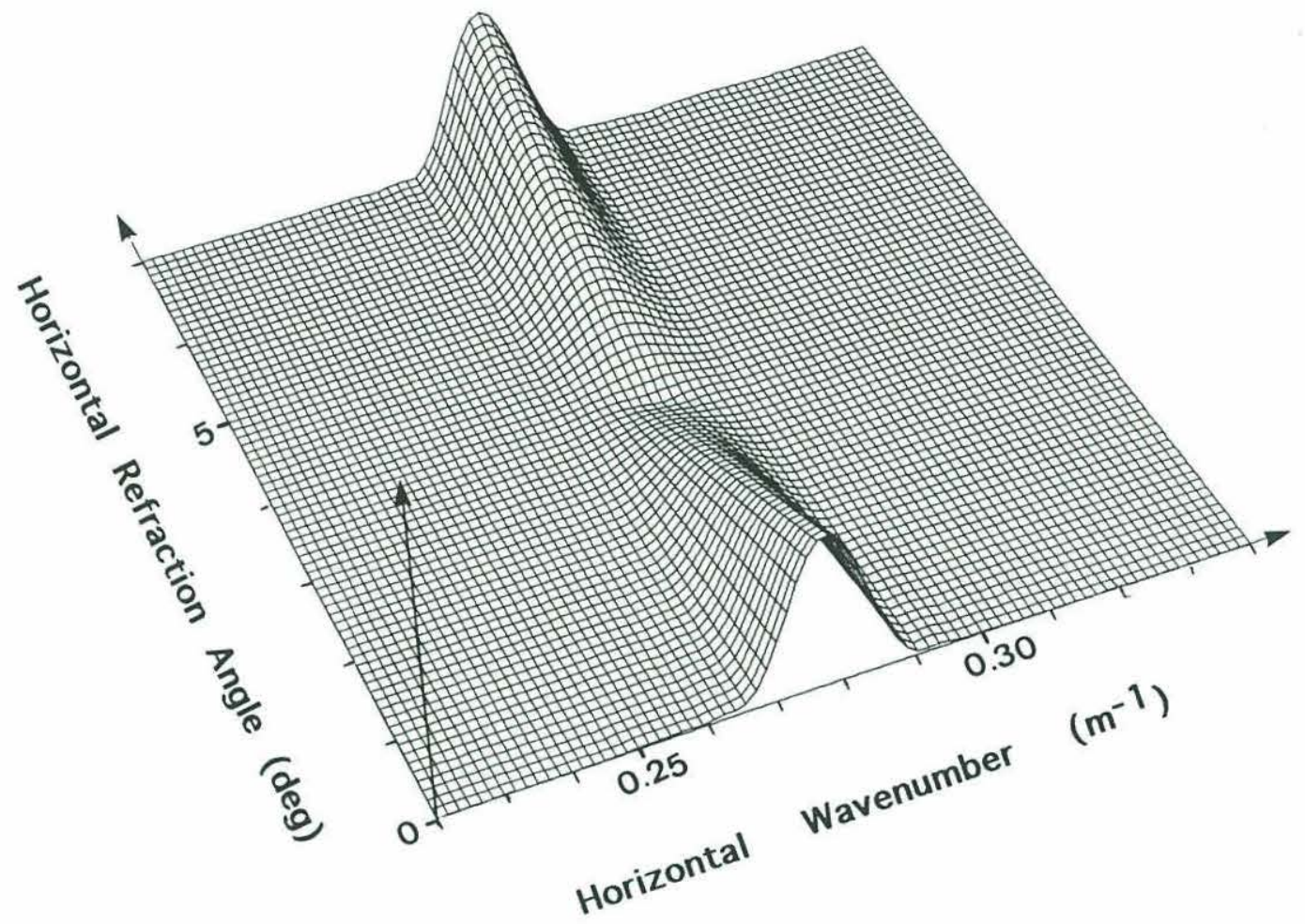

(b)

Figure 4-13, continued. 


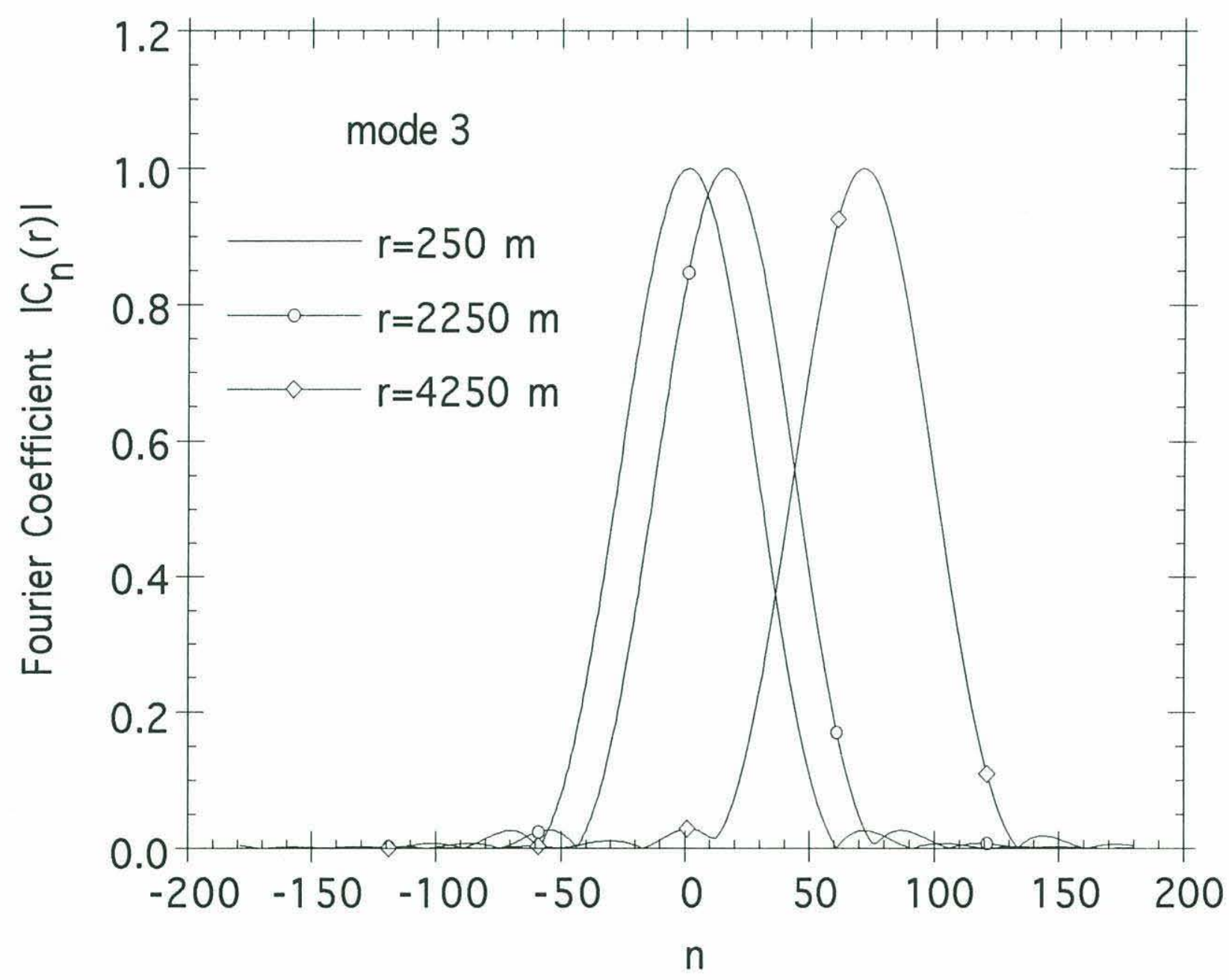

Figure 4-14: Comparison of the Fourier coefficients $\left|C_{n}(r ; \hat{\theta})\right|$ at the different ranges when setting the center direction of the Hanning window $v_{\Omega}$ as $\hat{\theta}=70^{\circ}$. The pressure field for the third mode has been separated by mode filtering. 


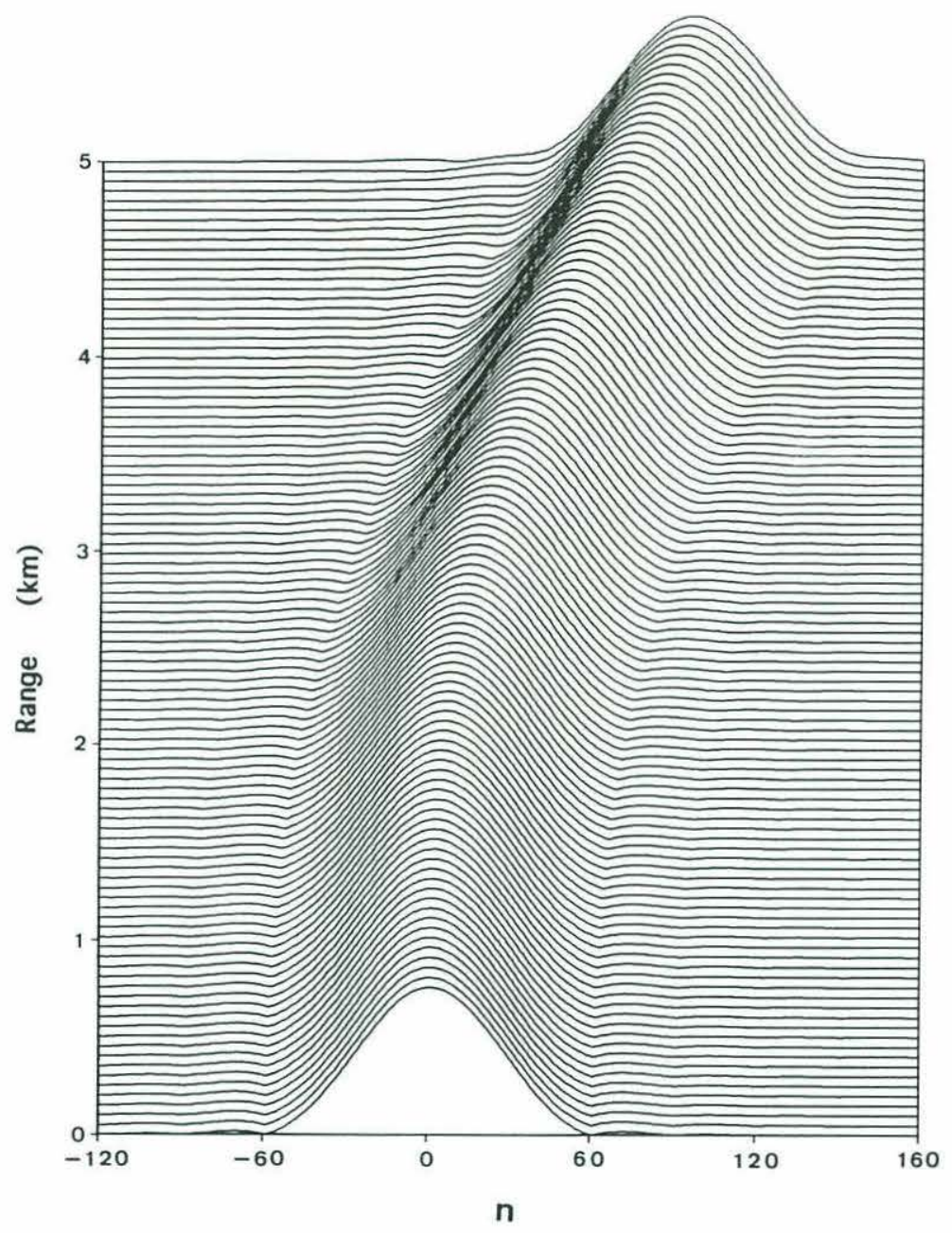

Figure 4-15: Range evolution of the Fourier coefficient $\left|C_{n}(r ; \hat{\theta})\right|$ along the radial with the fixed azimuth $\hat{\theta}=70^{\circ}$. The pressure field for the third mode has been separated by mode filtering. 


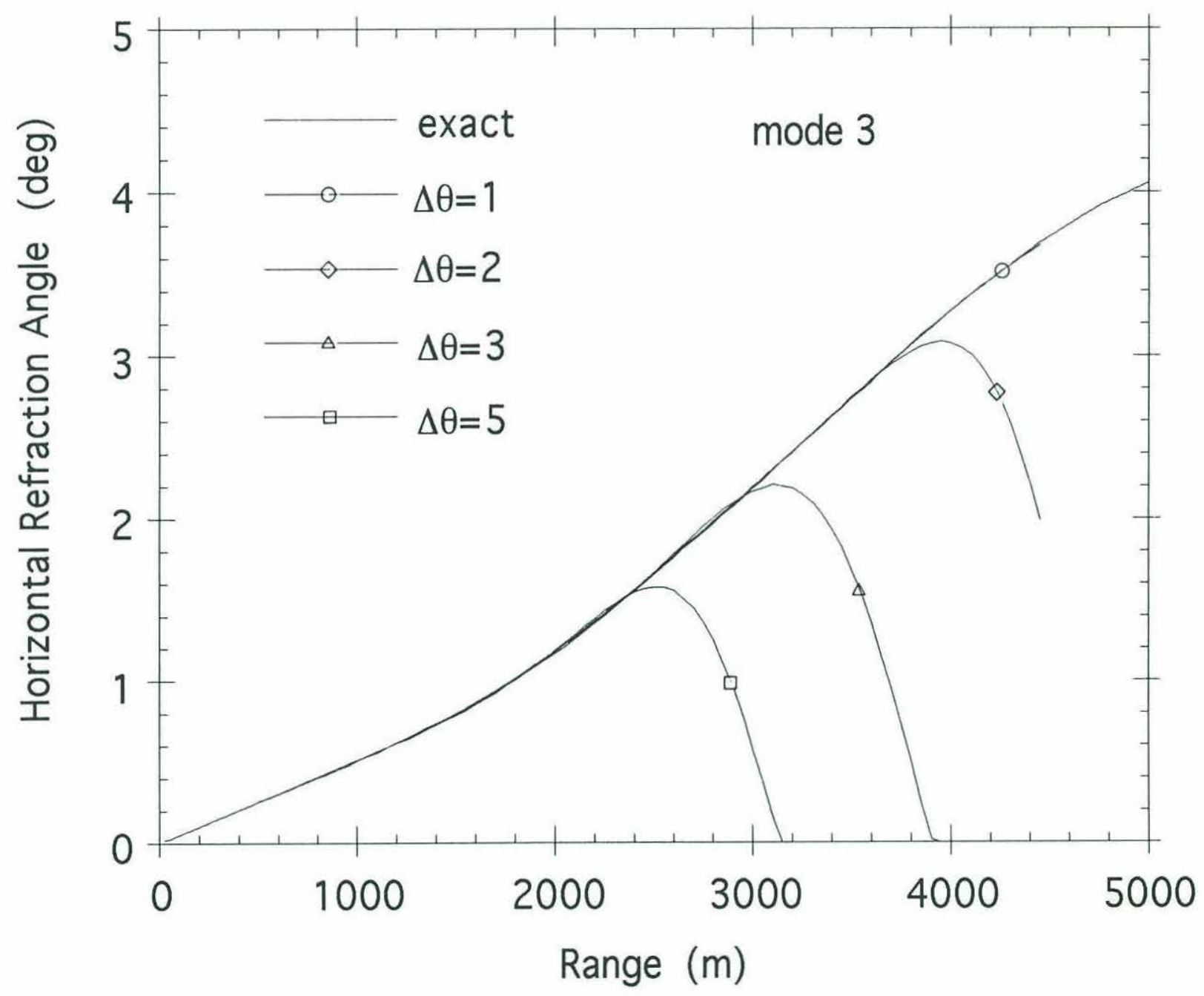

Figure 4-16: Comparison of the angles $\delta_{H}$ satisfying Eq.(4-27) for different sampling widths $\Delta \theta$. The center direction of the Hanning window $v_{\Omega}$ is set at $\hat{\theta}=70^{\circ}$. The exact angle $\left(\delta_{n}\right)$ of the horizontal ray direction is also shown. 
In Figure 4-17 we compare the results for different window widths with a fixed $\Delta \theta\left(=2^{\circ}\right)$. We cannot find a substantial difference among them at the range which satisfies the Nyquist criterion. On the other hand, when the range gets close to the maximum, the result shows that the narrow window width loses its precision faster than the wide window.

As discussed in Chapter 4.2.3, we can compensate for the underestimated peak position by using a scale factor $1-\frac{1}{2} \delta_{H}^{2}$, where the refraction angle has been obtained above. Figure 4-18 shows a comparison of the compensated amount in a wavenumber using $\delta_{H}$ and the exact wavenumber, i.e., $\kappa_{n}-\kappa_{n} \cos \delta_{n}$. In the same figure, the results for different $\Delta \theta$ are also compared. Due to the failure in the determination of $\delta_{H}$ as seen from Figure 4-17, we have an incorrect result beyond the maximum range which depends on $\Delta \theta$.

In the final stage, we apply the alternative method discussed in Chapter 4.3 to the simulated field. In this method, it is assumed that we can obtain the phase difference of the output signals of the mode filter which is applied to the pressure field along adjacent radials separated by the azimuthal width $\Delta \theta$. Figure 4-19 shows the results of Eq.(4-53), i.e., $\kappa_{n}(\hat{r})-\bar{\kappa}_{n}(\hat{r})$, for the different azimuthal widths $\Delta \theta$. In this figure, the exact wavenumber $\kappa_{n}-\kappa_{n} \cos \delta_{n}$ is also compared. It can be observed that the error increases with increasing width $\Delta \theta$. As seen from a comparison of the two curves of $\Delta \theta=55^{\circ}-70^{\circ}$ and $85^{\circ}-70^{\circ}$, the error has a different range dependence even for the same width $|\Delta \theta|$. This is due to the fact that the modal phase front has a different behavior in accordance with the local characteristics of the ocean environment, as shown in Figure 4-6.

As seen from a comparison of Figure 4-18 and Figure 4-19, both methods provide close results for determining the deficit in the wavenumber, as long as the observation range is within the maximum range associated with the Nyquist criterion.

In the next section, let us examine the relation between this maximum range and the sampling width in the azimuthal direction. 


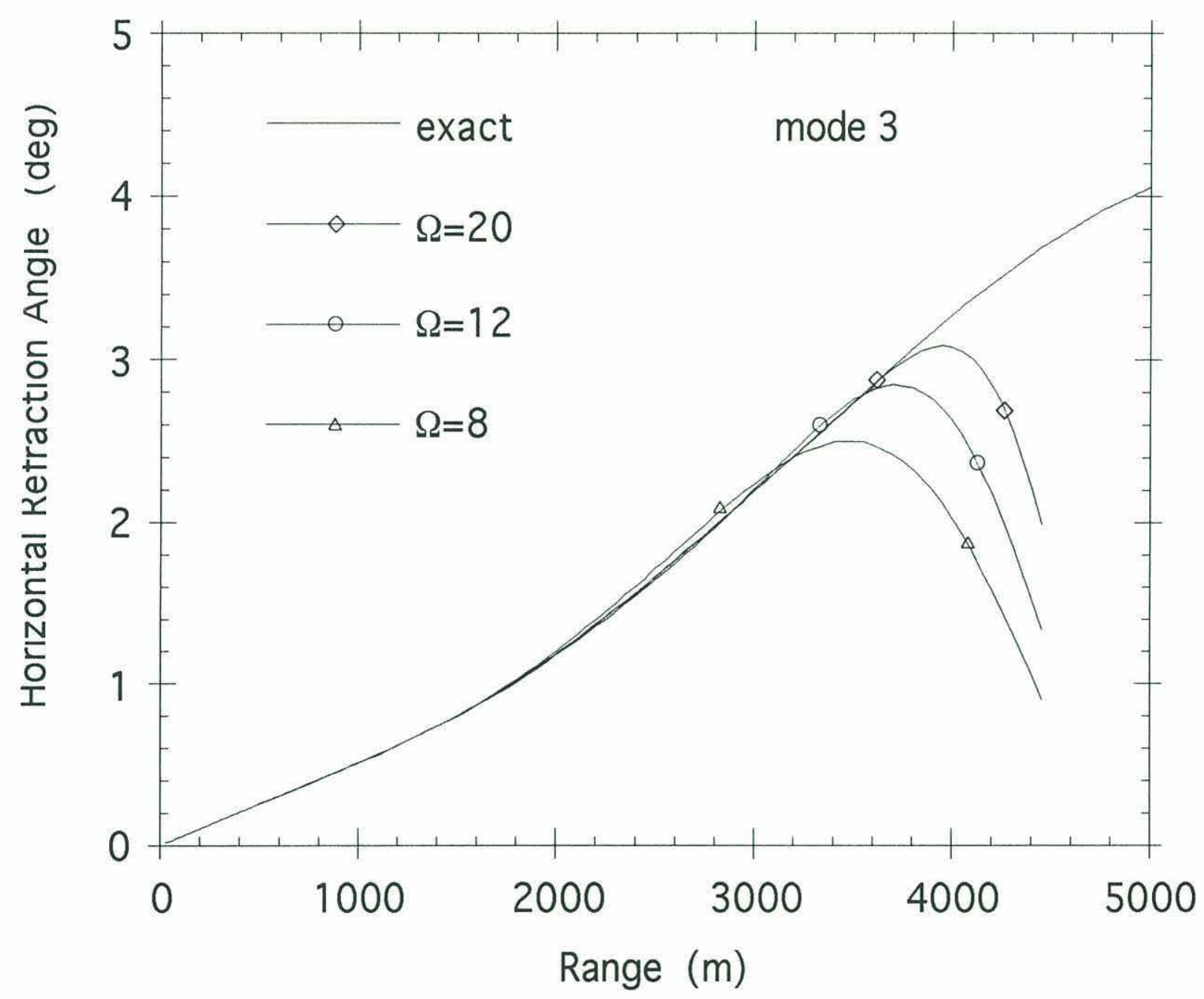

Figure 4-17: Comparison of the angles $\delta_{H}$ satisfying Eq.(4-27) for the different widths $\Omega$ of the Hanning window $v_{\Omega}$. The sampling width is set at $\Delta \theta=2^{\circ}$ and the center direction of the window is set at $\hat{\theta}=70^{\circ}$. The exact angle $\left(\delta_{n}\right)$ of the horizontal ray direction is also shown. 


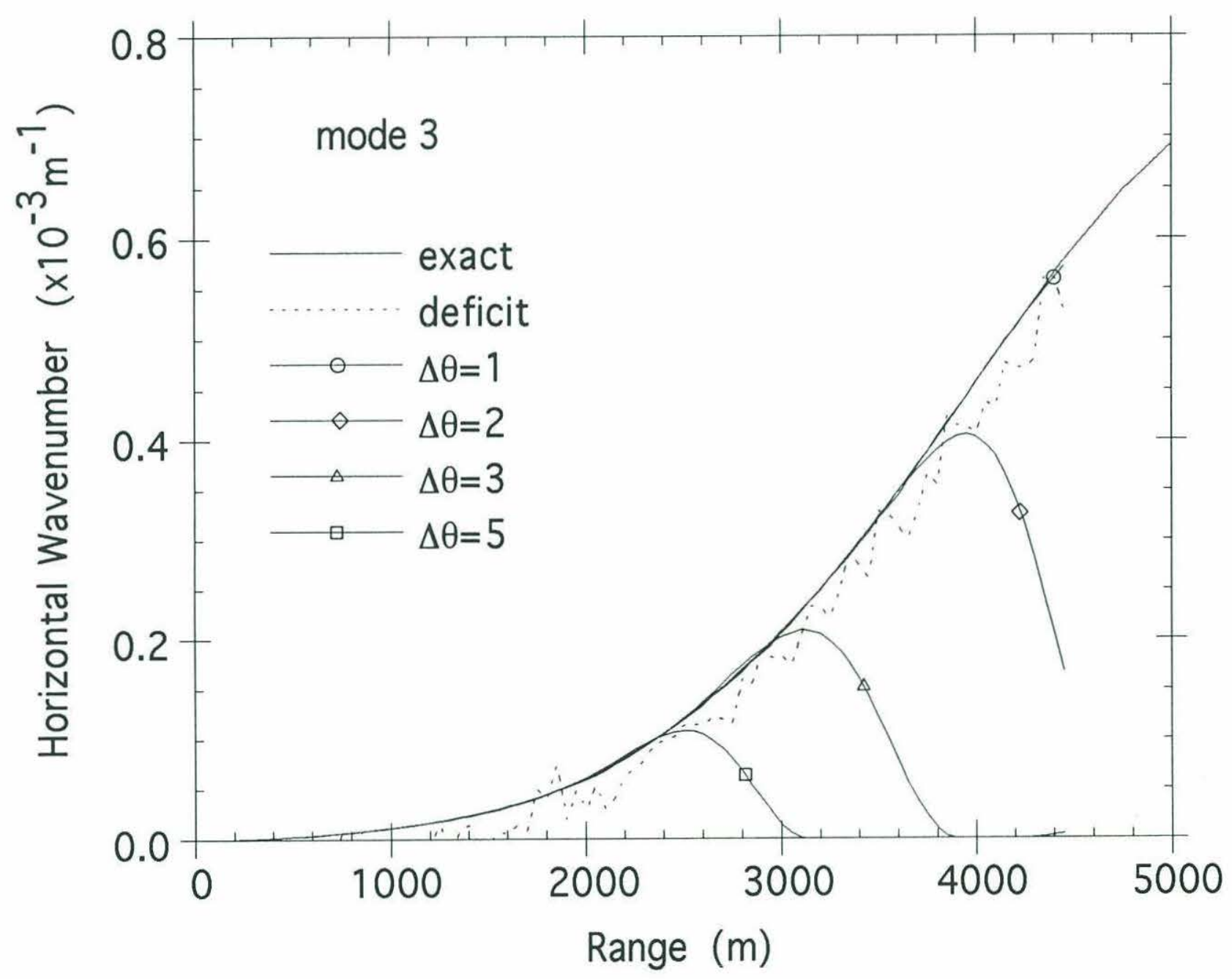

Figure 4-18: Comparison of the deficits in the wavenumber for the different sampling widths $\Delta \theta$. The center direction of the Hanning window $v_{\Omega}$ is set at $\hat{\theta}=70^{\circ}$. Both exact values $\left(\kappa_{n}-\kappa_{n} \cos \delta_{n}\right)$ and deficits in Figure 4-11 are also compared. 


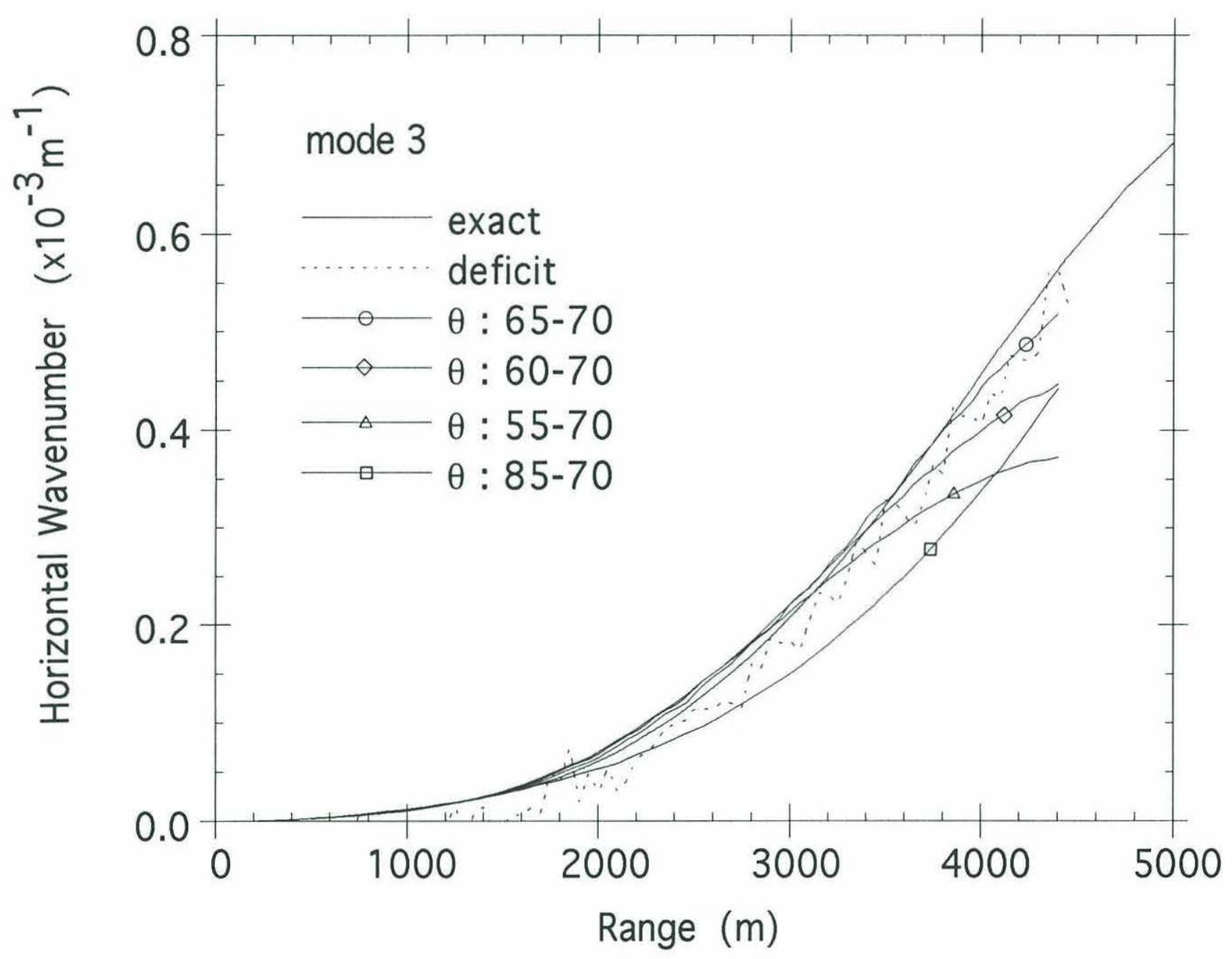

Figure 4-19: Comparison of the compensated amounts $\beta_{n}^{2} / 2 \bar{\kappa}_{n}$ in Eq.(4-53) for the different radials. The center direction of the Hanning window $v_{\Omega}$ is set at $\hat{\theta}=70^{\circ}$. Exact values $\left(\kappa_{n}-\kappa_{n} \cos \delta_{n}\right)$ and deficits in Figure 4-11 are also compared. 


\subsection{Experimental Design}

In this section, we will present an experimental design for measuring effectively the pressure field in a 3-D varying environment that also copes with the horizontal refraction effect.

As discussed in Section 4.2, unless the horizontal refraction is negligibly weak in a 3-D varying environment, we need to take into consideration this effect by using the general Hankel transform with a sliding window. In this transform, we first need to calculate the Fourier coefficients $C_{n}(r ; \hat{\theta})$ in Eq.(4-6), which is given by executing the integral with respect to $\theta$. Thus if the pressure field $p(r, \theta)$ is given in the azimuthal direction in addition to the radial direction, then the coefficient can be immediately calculated.

The pressure $p(r, \theta)$, however, cannot be provided continuously as a function of both range $r$ and azimuthal angle $\theta$ due to limitations in measurements made in the ocean. Namely, we can measure the field only along some transect sampled by a moving ship or a buoy. As discussed in the beginning of this chapter, since the phase front of pressure propagates mainly in the radial direction in the region of interest, the measurement should basically be carried out in the radial direction at selected azimuth angles $\theta_{i}$ (Figure 4-20(a)).

We have to then calculate $C_{n}(r ; \hat{\theta})$ using only the discrete values of the pressure field $p\left(r, \theta_{i}\right)(i=1, \ldots, I)$ in the azimuthal direction. Since we can, however, execute the integration for $C_{n}(r ; \hat{\theta})$ in Eq.(4-6) by using the FFT, the issue to resolve lies in the sampling distance $r \Delta \theta_{i}$. In order to avoid aliasing errors, this distance has to be less than one-half of the wavelength $\lambda_{\ell} / 2$, where $\lambda_{\ell}$ is defined as

$$
\lambda_{\ell}=\frac{2 \pi}{k_{\ell}}=\frac{2 \pi}{\kappa_{n} \sin \delta_{M}} .
$$


Here $\delta_{M}$ is the maximum refraction angle in the region of interest and $\kappa_{n}$ is the local eigenvalue of the $n$th mode (Figure 4-3). The above spatial Nyquist condition is expressed then as

$$
\Delta \theta_{i}<\frac{\pi}{r \kappa_{n} \sin \delta_{M}}
$$

Here we assume that we can incorporate data from the vertical array of receivers at $r=0$ in order to separate the pressure field by modes. If this is not so, $\kappa_{n}$ has to be replaced with $\omega / c_{w}$, where $c_{w}$ is the minimum sound speed in the profile. In general, we can safely use the minimum sound speed in the water column, yielding

$$
\Delta \theta_{i}<\frac{c_{w}}{2 r f \sin \delta_{M}}
$$

where $f$ is an acoustic frequency. In Eq.(4-56), we do not know the exact $\delta_{M}$, but we can estimate it by using the horizontal ray method along with an assumed sedimentary or rigid bottom.

As seen from these results, we have to decrease $\Delta \theta_{i}$ while increasing the range $r$. For example, we can set the measurement transects in the ocean as shown in Figure 420(b).

In general, the higher the mode, the stronger the horizontal refraction. Namely, $\delta_{M}$ has a larger value for the higher modes in Eq.(4-55). Therefore, we have to use smaller $\Delta \theta_{i}$ for those modes at long ranges. Since, however, the higher modes attenuate faster than the lower modes, we do not have to design the measurement strategy for the higher modes at long ranges.

As an alternative method with respect to the issues discussed above, but in a more approximate way, we first calculate $C_{n}\left(r_{j} ; \hat{\theta}\right)$ at selected ranges $r_{j}$ by using the pressure field $p\left(r_{j}, \theta\right)(j=1, \ldots, J)$ measured along an arc at these ranges; then, $C_{n}(r ; \hat{\theta})$ can be 


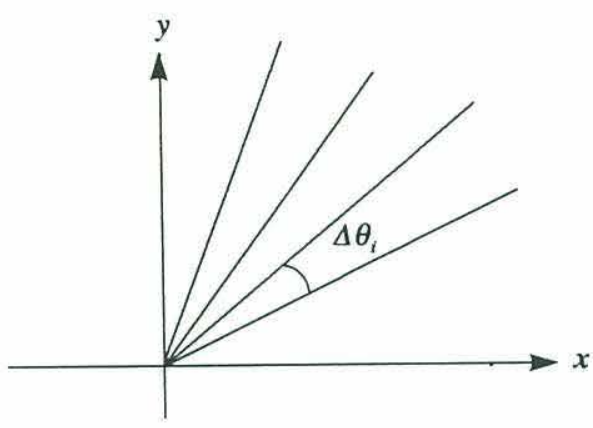

(a)

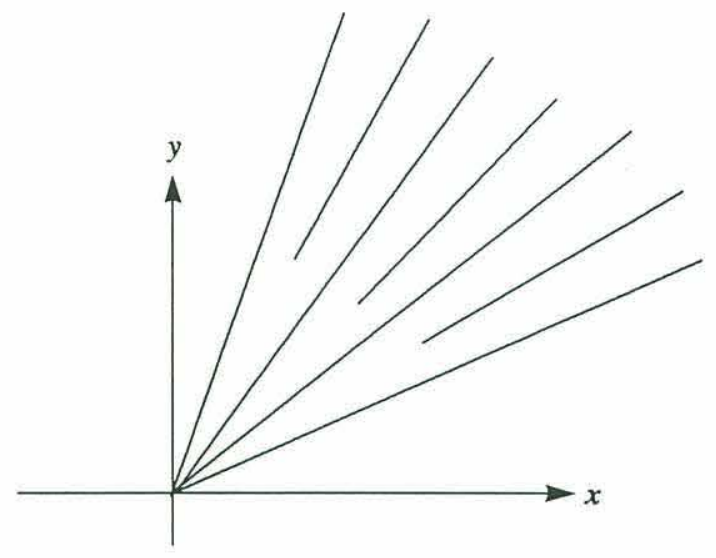

(b)

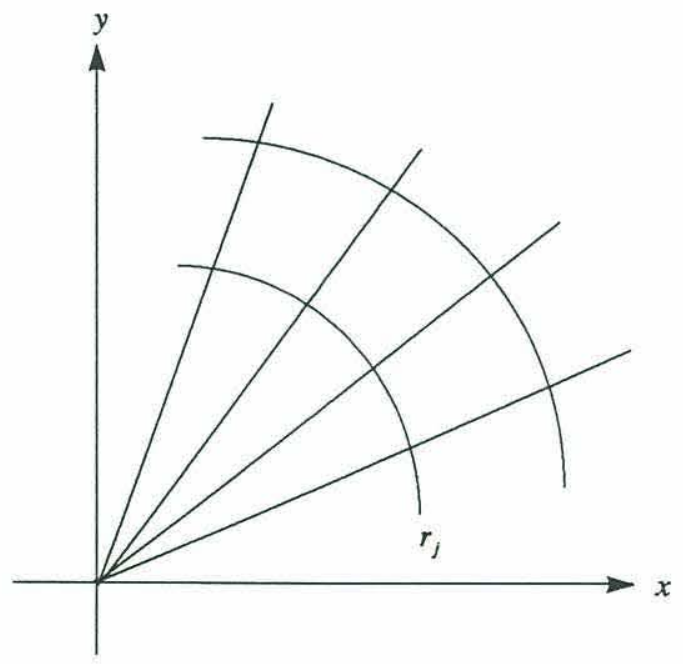

(c)

Figure 4-20: Design of transects for measuring the sound field in the ocean. 
interpolated between those ranges as shown in Figure 4-20(c). To employ this approach, the single mode environment based on mode filtering would be preferable to increase the precision of the interpolation.

Finally, if the horizontal refraction angle is negligibly small, i.e., $\delta_{M} \ll<1$, then we do not have to use the general Hankel transform and can apply the zero-order Hankel transform to the field in the radial direction of interest in the same way as shown in Chapter 3.

\subsection{Summary}

In an attempt to detect the local eigenvalues in a 3-D varying shallow water waveguide, we introduced a sliding window in a 2-D Fourier transform based on a cylindrical coordinate system and then studied the effect of horizontal refraction on the determination of the local eigenvalues.

In Section 4.1, we discussed issues arising from horizontal refraction in a 3-D varying waveguide. When applying the zero-order Hankel transform with a sliding window to the pressure field measured along a radial, the wavenumber determined from the peak position in the output spectrum has a deficit due to horizontal refraction.

In Section 4.2, we derived the general Hankel transform with a sliding window from the 2-D Fourier transform. By expanding it in a Taylor series, we found that the first term corresponds to the zero-order Hankel transform with a sliding window, which was obtained for the cylindrically symmetric waveguide in Chapter 3; the rest of the terms correspond to the zero-order Hankel transform of the pressure field differentiated with respect to the azimuthal angle.

In order to analyze the effect of horizontal refraction on the Hankel transform, we utilized the stationary phase method and evaluated the transform in an approximate manner. It was then found that the Fourier coefficients play the role of a filter for the variable $\varphi$ to match the direction of the major phase front associated with a mode. Based 
on this analysis, we can determine the horizontal refraction angle approximately by taking the variation of this transform with respect to $\varphi$. Then, by changing the scale of the wavenumber with the use of the determined refraction angle, it was shown that we can compensate for the deficit in the wavenumber obtained when using the zero-order Hankel transform with a sliding window in a 3-D varying waveguide.

In Section 4.3, in order to re-examine analytically the effect of horizontal refraction, we derived an alternative representation for a 2-D Fourier transform by using the phase difference between the outputs of the mode filter which was applied to the pressure field in the different azimuthal directions. By comparing the resulting transform with the zeroorder asymptotic Hankel transform, we demonstrated that the compensated amount for the local eigenvalue in the latter transform can be determined by using the phase difference in mode filtering.

In Section 4.4, we examined numerically the horizontal refraction effect by applying the transforms obtained in Section 4.2 and 4.3 to the pressure field that is simulated using the horizontal ray method. It was shown that we can determine the horizontal refraction angle by using the general Hankel transform, as long as the pressure field is sampled in the azimuthal direction so as to satisfy the spatial Nyquist criterion. We also showed that the deficit in the wavenumber can be determined by using these transforms.

In Section 4.5, we considered a method for the efficient measurement of the pressure field in a 3-D varying shallow water waveguide to deal with the horizontal refraction effect. Based on the spatial Nyquist criterion, the sampling width in the azimuthal angle for the general Hankel transform to work properly was determined in connection with the maximum range.

So far we have tried to detect the local eigenvalues in horizontally varying environments in Chapter 3 and Chapter 4. In the next chapter, we will use those local eigenvalues as input data to a method for determining the range-dependent geoacoustic properties. 


\section{Chapter 5 \\ Inverse Method for the Local Bottom Properties}

The purpose of this chapter is to develop a method for determining the local bottom properties from the local eigenvalues, which were estimated by using the Hankel transform with a sliding window in Chapters 3 and 4.

In the first place, we will try to understand how the range variation of the local bottom properties in the horizontal direction affects the range variation of the local eigenvalues. In order to analyze the relation between these two variations, we begin by setting up the bottom sediment model in the following section. Based on this bottom model, we derive the relation between the perturbed local eigenvalue and the perturbed geoacoustic parameters in Section 5.2. To verify this perturbation relation, we apply it to a range-dependent Pekeris model in Section 5.3. In Section 5.4, by representing the sound speed profile in each sediment layer as an $n^{2}$-linear curve, we express the perturbation relation more explicitly in terms of sediment parameters. In section 5.5, based on the perturbation relation, we develop an inverse method for determining the bottom parameters from the local eigenvalues. In Section 5.6, we apply the inverse method to the shallow water model used in Chapter 5.3. We show that the geoacoustic parameters can then be numerically obtained by solving the perturbation equation iteratively with range.

\subsection{Geoacoustic model}

From past studies, it is well known that the ocean bottom is multilayered structure and some experiments show the range-dependent variation of the geoacoustic properties in the sediment $[81,82]$. With these studies in mind, we set up a shallow water bottom 
model composed of a range-dependent, multilayered fluid medium, as shown in Figure 5-1. We use this bottom model throughout the chapter and develop an inverse method based on it.

In this bottom model, the sediment in each layer $(L \leq j \leq M)$ is assumed to have different physical properties, so that the sound speed, the density, and the attenuation coefficient may be discontinuous across each layer interface. Within each layer, however, the sound speed is assumed to vary in a continuous manner with respect to space. This variation is arbitrary in the vertical direction but gradual in the horizontal direction to meet the adiabatic condition. On the other hand, both the density and attenuation coefficient are assumed to be constant in each layer, but they may take on different values in different layers.

Since the local eigenvalues are provided along the radial as discussed in Chapter 4, the geoacoustic properties determined inversely from these eigenvalues are limited to ones along this radial only. Hence the azimuthal angle is only a parameter for distinguishing the radial direction and so the functional notation regarding an azimuth will be omitted throughout this chapter.

The layer interface $h_{j}(r)(1 \leq j \leq M)$, therefore, is expressed as a function of range only. In particular, the sea surface interface $h_{1}$ is always set to be zero in our model, and $h_{L}(r)$ corresponds to the bottom interface. Thus the layers $j=1$ through $j=L-1$ are located in the water column. Since the physical properties in the water column are considered to vary continuously, we do not need a layered structure in the water column in general. If we use these criteria, we can set $L=2$. If there exists, however, a water body with different densities, then it would be convenient to represent it using a layered structure. In any case, the density must be constant in each layer in the model.

For the same reasons mentioned above, the sound speed is expressed as a function of range and depth only, i.e., $c(z, r)$. In order for the adiabatic condition to be met, $|\partial c / \partial r|$ as well as $\left|d h_{j} / d r\right|$ have to be small enough to make the mode coupling coefficients small. 


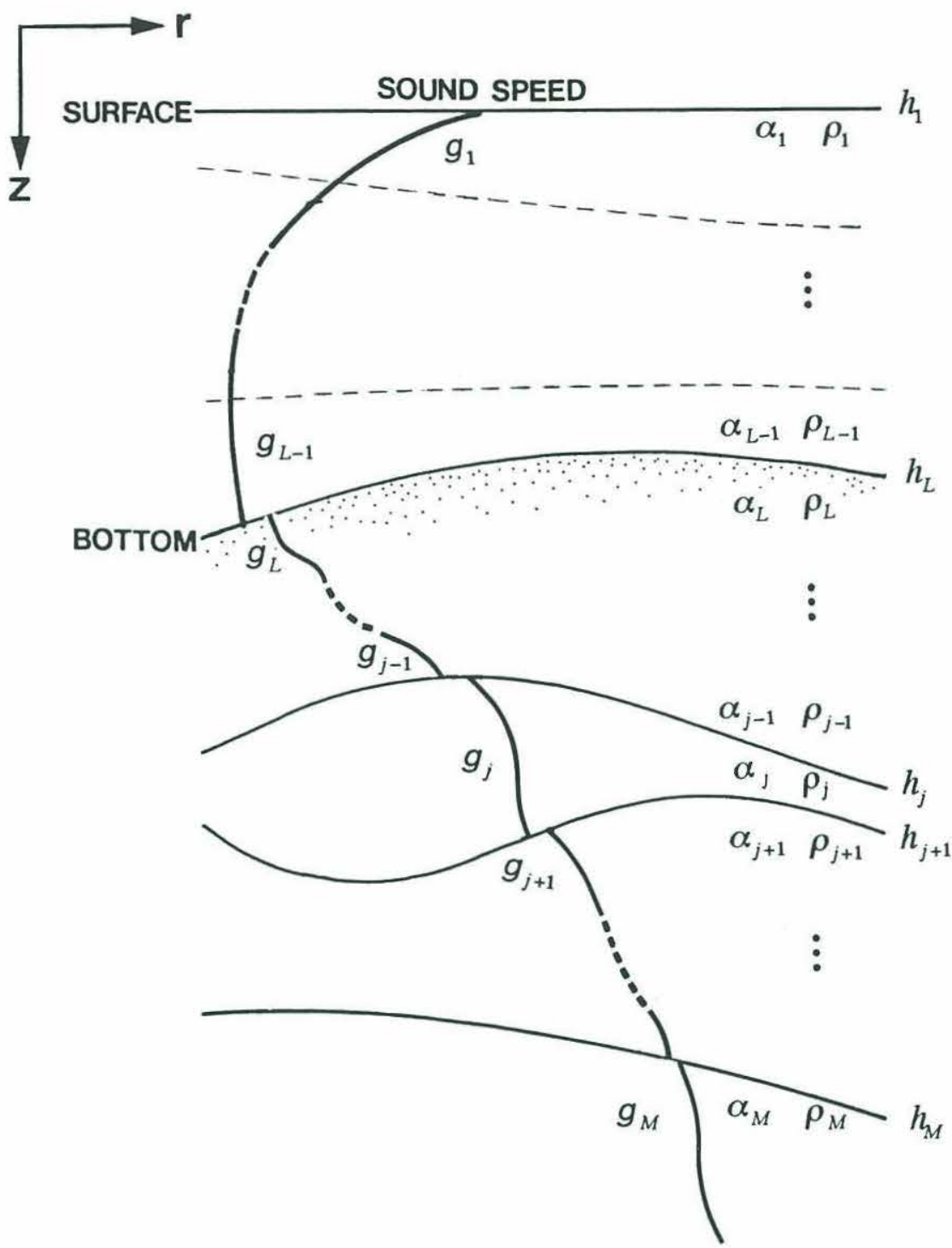

Figure 5-1: Shallow water model of non-stratified, multi-layer bottom sediment with horizontal changes in sound speed profile. 
As stated in Section 2.1, the branch line integral stems from the assumption of constant sound velocity in the lowest layer (half-space), i.e., the gradient $g_{M}=0$. If a small, positive number is assigned to $g_{M}$, then this branch line does not emerge and all modes become discrete; therefore, to avoid the difficulty arising from conversion between discrete and continuous modes in the propagation process, we assume $g_{M}>0$ in this model.

Based on this multilayered sediment bottom model, we will develop a method for determining the geoacoustic properties in the remaining sections using a perturbative approach.

\subsection{Relation between perturbed local eigenvalues and perturbed local bottom properties}

In this section, we will derive a relationship between perturbed local eigenvalues and perturbed geoacoustic parameters by using linear perturbation theory $[43,47]$.

With the geoacoustic model described in the previous section, we can utilize adiabatic mode theory, as long as the sound speed in the water column varies gradually. Since the density is assumed to be constant in each sediment layer, it is immediately seen from Eq.(2-8) that the local eigenfunctions $u_{n}$ satisfy

$$
\frac{\partial^{2} u_{n}}{\partial z^{2}}+\left[k^{2}(r, z)-\kappa_{n}^{2}(r)\right] u_{n}(r, z)=0 \quad .
$$

The local eigenfunctions $u_{n}$ also satisfy the orthonormality condition

$$
\int_{0}^{\infty} \frac{1}{\rho(r, z)} u_{n}(r, z) u_{m}(r, z) d z=\delta_{n m}
$$

and the following boundary conditions:

$$
\left.u_{n}(r, z)\right|_{z=0}=0,
$$


and

$$
\lim _{z \rightarrow \infty} \frac{\partial u_{n}}{\partial z}=0
$$

Eq.(5-3) represents a simple pressure-release condition at the sea surface. Thus surface roughness is ignored here, which should not incur a large error for low-frequency modes. On the other hand, the boundary condition at infinite depth is based on the assumption of a positive gradient in the lowest layer. If $g_{M}=0$ instead, then we confine ourselves to trapped modes only, so that the condition in Eq.(5-4) is still satisfied.

In addition to the above conditions, both pressure and vertical particle velocity must be continuous across each layer interface:

$$
\left.u_{n}\right|_{z=h_{j}-0}=\left.u_{n}\right|_{\varepsilon=h_{j}+0}
$$

and

$$
\left.\frac{1}{\rho_{j-1}} \frac{\partial u_{n}}{\partial z}\right|_{z=h_{j}-0}=\left.\frac{1}{\rho_{j}} \frac{\partial u_{n}}{\partial z}\right|_{z=h_{j}+0},
$$

where $h_{j}-0$ and $h_{j}+0$ refer to the limit approaching the layer interface $z=h_{j}$ from above and below, respectively. Although the continuity of particle velocity is originally defined in the direction normal to the interface in Eq.(5-6), the approximation made here leads to accurate results, as long as the interface varies gradually within the framework of the adiabatic approximation.

First, let us derive an integral version of Eq.(5-1), which is the basis for deriving the perturbation relation between the local eigenvalues and the geoacoustic properties [43]. Multiplying Eq. (5-1) by $u_{n} / \rho$, integrating over the entire depth, and integrating by parts in the first term yields 


$$
-\left.\frac{1}{\rho} u_{n} \frac{\partial u_{n}}{\partial z}\right|_{0} ^{\infty}+\int_{0}^{\infty} \frac{1}{\rho}\left(\frac{\partial u_{n}}{\partial z}\right)^{2} d z-\int_{0}^{\infty} \frac{1}{\rho} k^{2} u_{n}^{2} d z+\kappa_{n}^{2} \int_{0}^{\infty} \frac{1}{\rho} u_{n}^{2} d z=0
$$

Since the first term vanishes due to Eq.(5-3) and Eq.(5-4), we obtain the following form:

$$
\int_{0}^{\infty} \frac{1}{\rho}\left(\frac{\partial u_{n}}{\partial z}\right)^{2} d z-\int_{0}^{\infty} \frac{1}{\rho} k^{2} u_{n}^{2} d z+\kappa_{n}^{2} \int_{0}^{\infty} \frac{1}{\rho} u_{n}^{2} d z=0 .
$$

This form also provides a foundation for representing the group velocity associated with the $n$th mode in an integral form.

To accommodate the variation in interface depth $h_{j}(1 \leq j \leq M)$ in Eq.(5-8), let us rewrite the integration in Eq.(5-8) as $\int_{0}^{\infty} d z \rightarrow \sum_{j=1}^{M} \int_{h_{j}}^{h_{j+1}} d z$, that is

$$
\sum_{j=1}^{M} \frac{1}{\rho_{j}} \int_{h_{j}}^{h_{j+1}}\left(\frac{\partial u_{n}}{\partial z}\right)^{2} d z-\sum_{j=1}^{M} \frac{1}{\rho_{j}} \int_{h_{j}}^{h_{j+1}} k^{2} u_{n}^{2} d z+\kappa_{n}^{2} \sum_{j=1}^{M} \frac{1}{\rho_{j}} \int_{h_{j}}^{h_{j+1}} u_{n}^{2} d z=0,
$$

where $h_{M+1}=\infty$ has been set for notational convenience. Noting that each integration interval corresponds to a layer $\left(h_{j} \leq z \leq h_{j+1}\right)$, we have placed $\rho(z)$ outside of the integral in Eq.(5-9), because the density is assumed to be constant within each layer in the model.

In an attempt to derive a perturbation relation between the local eigenvalues and the geoacoustic parameters, we will perturb Eq.(5-9). When the medium (both water and bottom) changes with range, i.e., $k \rightarrow k+\Delta k$ and $h_{j} \rightarrow h_{j}+\Delta h_{j}$ as $r \rightarrow r+\Delta r$, the local eigenvalues and local eigenfunctions are also subject to change, i.e., $\kappa_{n} \rightarrow \kappa_{n}+\Delta \kappa_{n}$ and $u_{n} \rightarrow u_{n}+\Delta u_{n}$, thereby making Eq.(5-9) change as described below.

In order to avoid confusion in the mathematical development, we will derive the perturbation of Eq.(5-9) term-by-term and present each perturbation separately. Later we will combine all the perturbations. 
1. Range variation of the first integral in Eq.(5-9)

Taking a perturbation of the first term in Eq.(5-9) yields

$$
\begin{aligned}
& \sum_{j=1}^{M} \frac{1}{\rho_{j}} \int_{h_{j}+\Delta h_{j}}^{h_{j+1}+\Delta h_{j+1}}\left[\frac{\partial\left(u_{n}+\Delta u_{n}\right)}{\partial z}\right]^{2} d z \\
& \quad=\sum_{j=1}^{M} \frac{1}{\rho_{j}}\left(\int_{h_{j}+\Delta h_{j}}^{h_{j}}+\int_{h_{j}}^{h_{j+1}}+\int_{h_{j+1}}^{h_{j+1}+\Delta h_{j+1}}\right)\left(\frac{\partial u_{n}}{\partial z}+\frac{\partial \Delta u_{n}}{\partial z}\right)^{2} d z .
\end{aligned}
$$

If we confine ourselves to first order perturbation, then it follows that the left-hand side of Eq. $(5-10)$ becomes

$$
\begin{aligned}
& \sum_{j=1}^{M} \frac{1}{\rho_{j}} \int_{h_{j}+\Delta h_{j}}^{h_{j+1}+\Delta h_{j+1}}\left[\frac{\partial\left(u_{n}+\Delta u_{n}\right)}{\partial z}\right]^{2} d z \\
& \quad=\sum_{j=1}^{M} \frac{1}{\rho_{j}}\left[-\left.\left(\frac{\partial u_{n}}{\partial z}\right)^{2}\right|_{h_{j}} \Delta h_{j}+\left.\left(\frac{\partial u_{n}}{\partial z}\right)^{2}\right|_{h_{j+1}} \Delta h_{j+1}+\int_{h_{j}}^{h_{j+1}} 2 \frac{\partial u_{n}}{\partial z} \frac{\partial \Delta u_{n}}{\partial z} d z+\int_{h_{j}}^{h_{j+1}}\left(\frac{\partial u_{n}}{\partial z}\right)^{2} d z\right] .
\end{aligned}
$$

If we rewrite the second term on the right-hand side of Eq.(5-11) using $j^{\prime} \equiv j+1$ as

$$
\left.\sum_{j=1}^{M} \frac{1}{\rho_{j}}\left(\frac{\partial u_{n}}{\partial z}\right)^{2}\right|_{h_{j+1}} \Delta h_{j+1}=\left.\sum_{j^{\prime}=2}^{M} \frac{1}{\rho_{j^{\prime}-1}}\left(\frac{\partial u_{n}}{\partial z}\right)^{2}\right|_{h_{j}} \Delta h_{j^{\prime}},
$$

where the $(\mathrm{M}+1)$ term has dropped out due to $\left.\frac{\partial u_{n}}{\partial z}\right|_{u_{u+1}}=0$ in Eq.(5-4), then the first two terms in Eq. $(5-11)$ become

$$
\sum_{j=1}^{M} \frac{1}{\rho_{j}}\left[-\left.\left(\frac{\partial u_{n}}{\partial z}\right)^{2}\right|_{h_{j}} \Delta h_{j}+\left.\left(\frac{\partial u_{n}}{\partial z}\right)^{2}\right|_{h_{j+1}} \Delta h_{j+1}\right]=\left.\sum_{j=2}^{M}\left(\rho_{j-1}-\rho_{j}\right)\left(\frac{1}{\rho} \frac{\partial u_{n}}{\partial z}\right)^{2}\right|_{h_{j}} \Delta h_{j} .
$$

Here the $\Delta h_{1}$-term has dropped out due to $\Delta h_{1}=0$. 
The third term in Eq.(5-11) can be integrated by parts:

$$
\begin{aligned}
& \sum_{j=1}^{M} \frac{1}{\rho_{j}} \int_{h_{j}}^{h_{j+1}} 2 \frac{\partial u_{n}}{\partial z} \frac{\partial \Delta u_{n}}{\partial z} d z \\
& \quad=\sum_{j=1}^{M} \frac{2}{\rho_{j}}\left(\left.\frac{\partial u_{n}}{\partial z} \Delta u_{n}\right|_{h_{j}} ^{h_{j+1}}-\int_{h_{j}}^{h_{j+1}} \frac{\partial^{2} u_{n}}{\partial z^{2}} \Delta u_{n} d z\right) \\
& \quad=2 \sum_{j=2}^{M}\left(\left.\Delta u_{n}\right|_{h_{j}-0}-\left.\Delta u_{n}\right|_{h_{j}+0}\right)\left(\left.\frac{1}{\rho} \frac{\partial u_{n}}{\partial z}\right|_{h_{j}}\right)-\sum_{j=1}^{M} \frac{2}{\rho_{j}} \int_{h_{j}}^{h_{j+1}} \frac{\partial^{2} u_{n}}{\partial z^{2}} \Delta u_{n} d z,
\end{aligned}
$$

where Eq.(5-6) has been used in the last equation. At this point, let us apply the following relation,

$$
\left.\Delta u_{n}\right|_{h_{j}-0}-\left.\Delta u_{n}\right|_{h_{j}+0}=-\left.\left(\rho_{j-1}-\rho_{j}\right)\left(\frac{1}{\rho} \frac{\partial u_{n}}{\partial z}\right)\right|_{h_{j}} \Delta h_{j}
$$

to the first term on the right-hand side in Eq.(5-14). The proof of Eq.(5-15) is provided in Appendix A. Eq. (5-14) is then rewritten as

$$
\sum_{j=1}^{M} \frac{1}{\rho_{j}} \int_{h_{j}}^{h_{j+1}} 2 \frac{\partial u_{n}}{\partial z} \frac{\partial \Delta u_{n}}{\partial z} d z=-\left.2 \sum_{j=2}^{M}\left(\rho_{j-1}-\rho_{j}\right)\left(\frac{1}{\rho} \frac{\partial u_{n}}{\partial z}\right)^{2}\right|_{h_{j}} \Delta h_{j}-\sum_{j=1}^{M} \frac{2}{\rho_{j}} \int_{h_{j}}^{h_{j+1}} \frac{\partial^{2} u_{n}}{\partial z^{2}} \Delta u_{n} d z \text {. }
$$

Thus substitution of both Eq.(5-13) and Eq.(5-16) into Eq.(5-11) yields:

$$
\begin{aligned}
& \sum_{j=1}^{M} \frac{1}{\rho_{j}} \int_{h_{j}+\Delta h_{j}}^{h_{j+1}+\Delta h_{j+1}}\left[\frac{\partial\left(u_{n}+\Delta u_{n}\right)}{\partial z}\right]^{2} d z \\
& \quad=-\left.\sum_{j=2}^{M}\left(\rho_{j-1}-\rho_{j}\right)\left(\frac{1}{\rho} \frac{\partial u_{n}}{\partial z}\right)^{2}\right|_{h_{j}} \Delta h_{j}-\sum_{j=1}^{M} \frac{1}{\rho_{j}}\left[2 \int_{h_{j}}^{h_{j+1}} \frac{\partial^{2} u_{n}}{\partial z^{2}} \Delta u_{n} d z+\int_{h_{j}}^{h_{j+1}}\left(\frac{\partial u_{n}}{\partial z}\right)^{2} d z\right] .
\end{aligned}
$$


2. Range variation of the second integral in Eq.(5-9)

By taking a perturbation of the second integral in Eq.(5-9), we find to first order that

$$
\begin{aligned}
& \sum_{j=1}^{M} \frac{1}{\rho_{j}} \int_{h_{j}+\Delta h_{j}}^{h_{j+1}+\Delta h_{j+1}}\left[(k+\Delta k)^{2}\left(u_{n}+\Delta u_{n}\right)^{2}\right] d z \\
& \quad=\sum_{j=1}^{M} \frac{1}{\rho_{j}}\left[-\left.\left(k^{2} u_{n}^{2}\right)\right|_{h_{j}} \Delta h_{j}+\left.\left(k^{2} u_{n}^{2}\right)\right|_{k_{j+1}} \Delta h_{j+1}+\int_{h_{j}}^{h_{j+1}}\left(2 k \Delta k u_{n}^{2}+k^{2} 2 u_{n} \Delta u_{n}+k^{2} u_{n}^{2}\right) d z\right] .
\end{aligned}
$$

Use of Eq.(5-5) after replacement of the index $(j+1 \rightarrow j)$ in the second term on the righthand side of Eq.(5-18) yields

$$
\begin{aligned}
& \sum_{j=1}^{M} \frac{1}{\rho_{j}} \int_{h_{j}+\Delta h_{j}}^{h_{j+1}+\Delta h_{j+1}}\left[(k+\Delta k)^{2}\left(u_{n}+\Delta u_{n}\right)^{2}\right] d z=\left.\sum_{j=2}^{M}\left(\frac{\left.k^{2}\right|_{h_{j}-0}}{\rho_{j-1}}-\frac{\left.k^{2}\right|_{j}+0}{\rho_{j}}\right) u_{n}^{2}\right|_{h_{j}} \Delta h_{j} \\
& \quad+\sum_{j=1}^{M} \frac{1}{\rho_{j}}\left[\int_{h_{j}}^{h_{j+1}} 2 k \Delta k u_{n}^{2} d z+\int_{h_{j}}^{h_{j+1}} 2 k^{2} u_{n} \Delta u_{n} d z+\int_{h_{j}}^{h_{j+1}} k^{2} u_{n}^{2} d z\right] .
\end{aligned}
$$

3. Range variation of the third integral in Eq.(5-9)

By taking a perturbation of the third integral in Eq.(5-9), we find to first order that

$$
\begin{aligned}
& \left(\kappa_{n}+\Delta \kappa_{n}\right)^{2} \sum_{j=1}^{M} \frac{1}{\rho_{j}} \int_{h_{j}+\Delta h_{j}}^{h_{j+1}+\Delta h_{j+1}}\left(u_{n}+\Delta u_{n}\right)^{2} d z=\kappa_{n}^{2} \sum_{j=1}^{M} \frac{1}{\rho_{j}}\left(-\left.u_{n}^{2}\right|_{h_{j}} \Delta h_{j}+\left.u_{n}^{2}\right|_{h_{j+1}} \Delta h_{j+1}\right) \\
& +2 \kappa_{n} \Delta \kappa_{n} \sum_{j=1}^{M} \frac{1}{\rho_{j}} \int_{h_{j}}^{h_{j+1}} u_{n}^{2} d z+\kappa_{n}^{2} \sum_{j=1}^{M} \frac{1}{\rho_{j}}\left[\int_{h_{j}}^{h_{j+1}}\left(2 u_{n} \Delta u_{n}+u_{n}^{2}\right) d z\right] .
\end{aligned}
$$

By using Eq. (5-5) after replacement of the index $(j+1 \rightarrow j)$ in the first term on the righthand side of Eq.(5-20), it follows that 


$$
\begin{aligned}
& \left(\kappa_{n}+\Delta \kappa_{n}\right)^{2} \sum_{j=1}^{M} \frac{1}{\rho_{j}} \int_{h_{j}+\Delta k_{j}}^{h_{j+1}+\Delta h_{j+1}}\left(u_{n}+\Delta u_{n}\right)^{2} d z \\
& =\left.\kappa_{n}^{2} \sum_{j=2}^{M}\left(\frac{1}{\rho_{j-1}}-\frac{1}{\rho_{j}}\right) u_{n}^{2}\right|_{h_{j}} \Delta h_{j}+2 \kappa_{n} \Delta \kappa_{n}+\kappa_{n}^{2} \sum_{j=1}^{M} \frac{1}{\rho_{j}}\left[2 \int_{h_{j}}^{h_{j+1}} u_{n} \Delta u_{n} d z+\int_{h_{j}}^{h_{j+1}} u_{n}^{2} d z\right] .
\end{aligned}
$$

Now, by substituting Eq.(5-17), Eq.(5-19) and Eq.(5-21) to Eq.(5-9), we obtain

$$
\begin{gathered}
2 \kappa_{n} \Delta \kappa_{n}-\sum_{j=2}^{M}\left[\left.\left(\frac{\left.\gamma_{n}^{2}\right|_{k_{j}-0}}{\rho_{j-1}}-\frac{\left.\gamma_{n}^{2}\right|_{h_{j}+0}}{\rho_{j}}\right) u_{n}^{2}\right|_{h_{j}}+\left.\left(\rho_{j-1}-\rho_{j}\right)\left(\frac{1}{\rho} \frac{\partial u_{n}}{\partial z}\right)^{2}\right|_{h_{j}}\right] \Delta h_{j} \\
-\sum_{j=1}^{M} \frac{2}{\rho_{j}} \int_{h_{j}}^{h_{j+1}} k \Delta k u_{n}^{2} d z-\sum_{j=1}^{M} \frac{2}{\rho_{j}} \int_{h_{j}}^{h_{j+1}}\left[\frac{\partial^{2} u_{n}}{\partial z^{2}}+\left(k^{2}-\kappa_{n}^{2}\right) u_{n}\right] \Delta u_{n} d z \\
+\left[\int_{0}^{\infty} \frac{1}{\rho}\left(\frac{\partial u_{n}}{\partial z}\right)^{2} d z-\int_{0}^{\infty} \frac{1}{\rho} k^{2} u_{n}^{2} d z+\kappa_{n}^{2} \int_{0}^{-} \frac{1}{\rho} u_{n}^{2} d z\right]=0,
\end{gathered}
$$

where $\gamma_{n} \equiv\left(k^{2}-\kappa_{n}^{2}\right)^{1 / 2}$ is the vertical wavenumber, and $\left.\gamma_{n}\right|_{h_{j}-0}$ and $\left.\gamma_{n}\right|_{k_{j}+0}$ stand for the wavenumbers on the upper and lower sides of $h_{j}$, respectively. The discontinuity in $\gamma_{n}$ at these interfaces $h_{j}$ originates from the discontinuity in the sound speed at $h_{j}$. At this point we find that the terms in the last two brackets in Eq.(5-22) become zero owing to Eq.(5-1) and Eq.(5-8), respectively. Both density and sound speed in the water column can be considered generally to change continuously, so that each coefficient of $\Delta h_{j}$ from $j=2$ through $j=L-1$ in Eq.(5-22) becomes zero. By splitting the integrals that include $\Delta k$ into the water region and the bottom region and dividing both sides of Eq. $(5-22)$ by $2 \kappa_{n}$, we can finally obtain 


$$
\begin{aligned}
\Delta \kappa_{n}= & \frac{1}{2 \kappa_{n}} \sum_{j=L}^{M}\left[\left.\left(\frac{\left.\gamma_{n}^{2}\right|_{h_{j}-0}}{\rho_{j-1}}-\frac{\left.\gamma_{n}^{2}\right|_{h_{j}+0}}{\rho_{j}}\right) u_{n}^{2}\right|_{h_{j}}+\left.\left(\rho_{j-1}-\rho_{j}\right)\left(\frac{1}{\rho} \frac{\partial u_{n}}{\partial z}\right)^{2}\right|_{h_{j}}\right] \Delta h_{j} \\
& +\frac{1}{\kappa_{n} \rho_{w}} \int_{0}^{h_{L}} k \Delta k u_{n}^{2} d z+\frac{1}{\kappa_{n}} \sum_{j=L}^{M} \frac{1}{\rho_{j}} \int_{h_{j}}^{h_{j+1}} k \Delta k u_{n}^{2} d z,
\end{aligned}
$$

where $\rho_{w}$ stands for the water density. If we use $2 \kappa_{n} \Delta \kappa_{n}=\Delta \kappa_{n}^{2}$ and $2 k \Delta k=\Delta k^{2}$, then Eq. (5-23) can be rewritten as

$$
\begin{aligned}
\Delta \kappa_{n}^{2}= & \sum_{j=L}^{M}\left[\left.\left(\frac{\left.\gamma_{n}^{2}\right|_{h_{j}-0}}{\rho_{j-1}}-\frac{\left.\gamma_{n}^{2}\right|_{h_{j}+0}}{\rho_{j}}\right) u_{n}^{2}\right|_{h_{j}}+\left.\left(\rho_{j-1}-\rho_{j}\right)\left(\frac{1}{\rho} \frac{\partial u_{n}}{\partial z}\right)^{2}\right|_{h_{j}}\right] \Delta h_{j} \\
& +\frac{1}{\rho_{w}} \int_{0}^{h_{L}} \Delta k^{2} u_{n}^{2} d z+\sum_{j=L}^{M} \frac{1}{\rho_{j}} \int_{h_{j}}^{h_{j+1}} \Delta k^{2} u_{n}^{2} d z .
\end{aligned}
$$

Both Eq.(5-23) and Eq.(5-24) show how the range variation of the local eigenvalue depends on the range variation of each sediment interface depth and the range variation of the wavenumber (sound speed) in each sediment layer. Thus these equations can reveal which layer interface and which portion of the sediment contributes to the range variation of the local modes. In the next section, we will apply Eq.(5-24) to a range-dependent Pekeris waveguide and will demonstrate that it provides an accurate result.

\subsection{Range-dependent Pekeris waveguide}

In this section, to check the validity of the resulting equation for $\Delta \kappa_{n}$ (Eq.(5-24)) obtained in the previous section, we will apply Eq.(5-24) to a range-dependent Pekeris waveguide [16,52], which can be regarded as the simplest case of the multilayered model we described in Section 5.1 except that here we assume $g_{M}=0$. If we, however, confine 
ourselves to trapped modes only, then the boundary condition in Eq.(5-4) is satisfied as seen from Eqs.(5-31) and (5-33), so that Eq.(5-24) is applicable to the Pekeris waveguide as well.

The advantage of using a Pekeris waveguide is that the characteristic equation is provided in a simple, analytical form and, consequently, the range variation of the local eigenvalues $\Delta \kappa_{n}$ can be obtained in closed form by directly differentiating the characteristic equation. This enables us to compare the two analytical forms for $\Delta \kappa_{n}$, which are derived in different ways.

Before deriving those forms, let us summarize the features of a range-dependent Pekeris waveguide. As illustrated in Figure 5-2, the sound speed in both the water column and bottom is taken to be constant vertically but is allowed to change in the horizontal direction such that

$$
c(r, z)= \begin{cases}c_{1}(r) & (0 \leq z \leq h(r)), \\ c_{2}(r) & (h(r) \leq z<\infty) .\end{cases}
$$

Since we are interested in trapped modes only, the following condition has to be met at each range:

$$
\frac{\omega}{c_{2}(r)}<\operatorname{Re}\left[\kappa_{n}(r)\right]<\frac{\omega}{c_{1}(r)} .
$$

The bathymetry is also range dependent, but the density in both regions remains constant:

$$
\rho(r, z)= \begin{cases}\rho_{1} & (0 \leq z \leq h(r)), \\ \rho_{2} & (h(r) \leq z<\infty) .\end{cases}
$$

In this Pekeris waveguide, the local eigenvalues satisfy the following characteristic equation obtained by using the boundary conditions at the sea surface and the waterbottom interface. 


$$
\gamma_{1 n} \cot \left(\gamma_{1 n} h\right)=i \varepsilon \gamma_{2 n}
$$

where $\varepsilon \equiv \rho_{1} / \rho_{2}$; and $\gamma_{1 n}$ and $\gamma_{2 n}$ are the vertical wavenumbers in the water column and bottom, respectively, and are defined by

$$
\begin{aligned}
& \gamma_{1 n}=\left(k_{1}^{2}-\kappa_{n}^{2}\right)^{\frac{1}{2}}, \\
& \gamma_{2 n}=\left(k_{2}^{2}-\kappa_{n}^{2}\right)^{\frac{1}{2}},
\end{aligned}
$$

with $k_{1} \equiv \omega / c_{1}$ and $k_{2} \equiv \omega / c_{2}$. In the case of trapped modes, $k_{2}<\kappa_{n}$ is satisfied from Eq.(5-26), so that Eq.(5-30) can be rewritten as

$$
\gamma_{2 n}=i \sqrt{\kappa_{n}^{2}-k_{2}^{2}}
$$

The local eigenfunction can also be determined so as to satisfy the boundary condition at the sea surface and the radiation condition at infinite depth, yielding

$$
\begin{aligned}
& u_{n}(r, z)=\left(\frac{2 \rho_{1} \gamma_{1 n}}{\gamma_{1 n} h-\sin \left(\gamma_{1 n} h\right) \cos \left(\gamma_{1 n} h\right)-\varepsilon^{2} \tan \left(\gamma_{1 n} h\right) \sin ^{2}\left(\gamma_{1 n} h\right)}\right)^{1 / 2} \sin \left(\gamma_{1 n} z\right) \\
& u_{n}(r, z)=\left(\frac{2 \rho_{1} \gamma_{1 n}}{\gamma_{1 n} h-\sin \left(\gamma_{1 n} h\right) \cos \left(\gamma_{1 n} h\right)-\varepsilon^{2} \tan \left(\gamma_{1 n} h\right) \sin ^{2}\left(\gamma_{1 n} h\right)}\right)^{1 / 2} \sin \left(\gamma_{1 n} h\right) e^{i \gamma_{2 n}(z-h)} \\
& (h<z),
\end{aligned}
$$

where the $u_{n}$ satisfy the orthonormality condition Eq.(5-2) along with Eq.(5-5) and Eq.(56) at $z=h(r)$. 


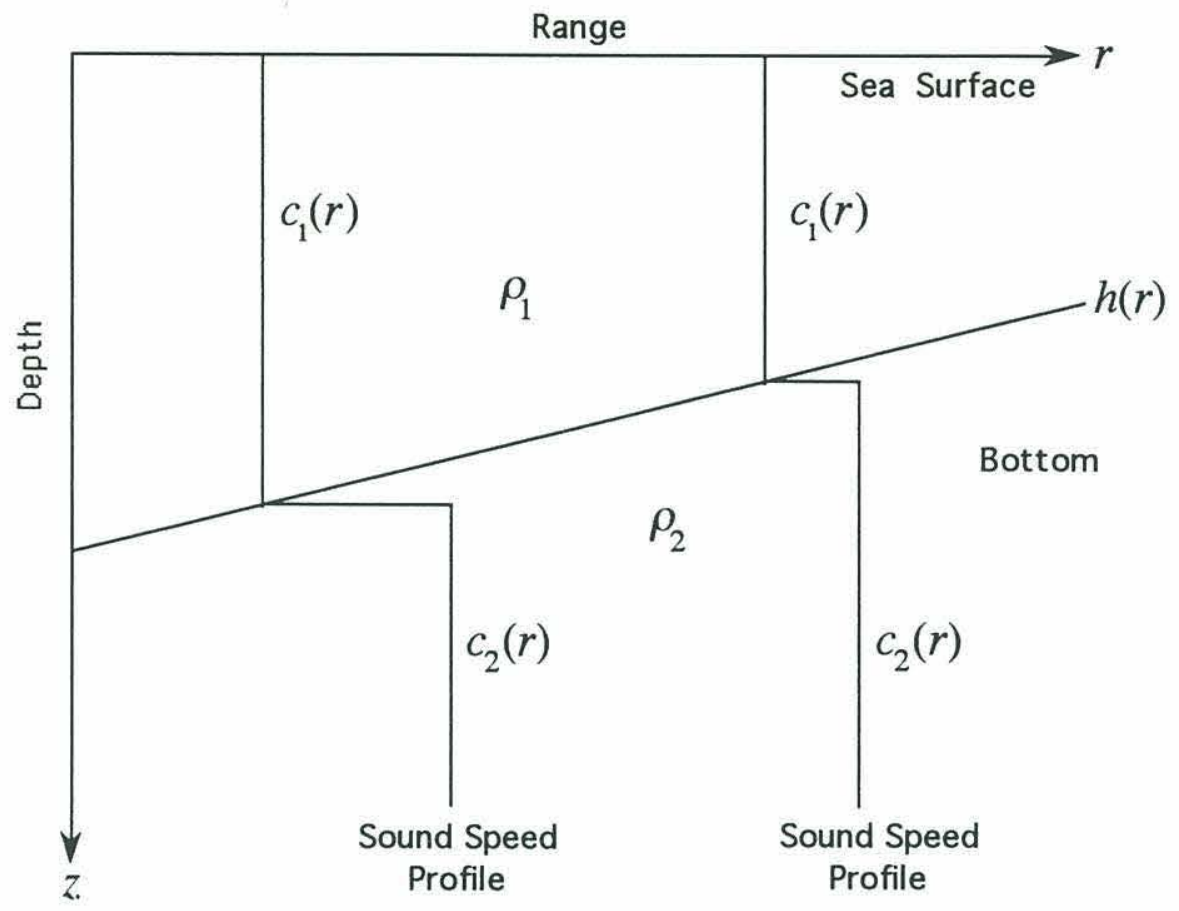

Figure 5-2: Range-dependent Pekeris waveguide. 


\subsubsection{Derivation of $\Delta \kappa_{n}$ by differentiation of the characteristic equation}

In this subsection, we will derive the analytical form for $\Delta \kappa_{n}$ directly from the characteristic equation given by Eq.(5-28).

Differentiating both sides of Eq.(5-28) with respect to $r$ gives

$$
\frac{d \gamma_{1 n}}{d r} \cot \left(\gamma_{1 n} h\right)+\gamma_{1 n} \frac{-1}{\sin ^{2}\left(\gamma_{1 n} h\right)}\left(\frac{d \gamma_{1 n}}{d r} h+\gamma_{1 n} \frac{d h}{d r}\right)=i \varepsilon \frac{d \gamma_{2 n}}{d r}
$$

Rearrangement of this equation yields

$$
\frac{d \gamma_{1 n}}{d r}\left(\cot \left(\gamma_{1 n} h\right)-\frac{\gamma_{1 n} h}{\sin ^{2}\left(\gamma_{1 n} h\right)}\right)-i \varepsilon \frac{d \gamma_{2 n}}{d r}=\frac{\gamma_{1 n}^{2}}{\sin ^{2}\left(\gamma_{1 n} h\right)} \frac{d h}{d r}
$$

In order to represent $d \gamma_{j n} / d r$ in Eq.(5-35) in terms of $d \kappa_{n} / d r$, let us also differentiate both Eq.(5-29) and (5-30) with respect to $r$ :

$$
\frac{d \gamma_{1 n}}{d r}=\frac{1}{2 \gamma_{1 n}}\left(\frac{d k_{1}^{2}}{d r}-\frac{d \kappa_{n}^{2}}{d r}\right)
$$

and

$$
\frac{d \gamma_{2 n}}{d r}=\frac{1}{2 \gamma_{2 n}}\left(\frac{d k_{2}^{2}}{d r}-\frac{d \kappa_{n}^{2}}{d r}\right)=\frac{i \varepsilon}{2 \gamma_{1 n} \cot \left(\gamma_{1 n} h\right)}\left(\frac{d k_{2}^{2}}{d r}-\frac{d \kappa_{n}^{2}}{d r}\right),
$$

where Eq. (5-28) has been used in (5-37). Thus substituting both Eqs.(5-36) and (5-37) into Eq.(5-35) yields

$$
\begin{gathered}
\frac{1}{2 \gamma_{1 n}}\left(\frac{d k_{1}^{2}}{d r}-\frac{d \kappa_{n}^{2}}{d r}\right)\left(\frac{\sin \left(\gamma_{1 n} h\right) \cos \left(\gamma_{1 n} h\right)-\gamma_{1 n} h}{\sin ^{2}\left(\gamma_{1 n} h\right)}\right)+\frac{\varepsilon^{2}}{2 \gamma_{1 n} \cot \left(\gamma_{1 n} h\right)}\left(\frac{d k_{2}^{2}}{d r}-\frac{d \kappa_{n}^{2}}{d r}\right) \\
=\frac{\gamma_{1 n}^{2}}{\sin ^{2}\left(\gamma_{1 n} h\right)} \frac{d h}{d r} .
\end{gathered}
$$


Rearrangement after multiplying both sides of Eq.(5-38) by $2 \gamma_{1 n} \sin ^{2}\left(\gamma_{1 n} h\right)$ yields

$$
\begin{aligned}
& {\left[\gamma_{1 n} h-\sin \left(\gamma_{1 n} h\right) \cos \left(\gamma_{1 n} h\right)-\varepsilon^{2} \tan \left(\gamma_{1 n} h\right) \sin ^{2}\left(\gamma_{1 n} h\right)\right] \frac{d \kappa_{n}^{2}}{d r}} \\
& \quad=2 \gamma_{1 n}^{3} \frac{d h}{d r}+\left[\gamma_{1 n} h-\sin \left(\gamma_{1 n} h\right) \cos \left(\gamma_{1 n} h\right)\right] \frac{d k_{1}^{2}}{d r}-\varepsilon^{2} \tan \left(\gamma_{1 n} h\right) \sin ^{2}\left(\gamma_{1 n} h\right) \frac{d k_{2}^{2}}{d r}
\end{aligned}
$$

Multiplying both sides of Eq.(5-39) by $\Delta r$ leads to the following form:

$$
\begin{aligned}
\Delta \kappa_{n}^{2}= & \frac{2 \gamma_{1 n}^{3}}{\gamma_{1 n} h-\sin \left(\gamma_{1 n} h\right) \cos \left(\gamma_{1 n} h\right)-\varepsilon^{2} \tan \left(\gamma_{1 n} h\right) \sin ^{2}\left(\gamma_{1 n} h\right)} \Delta h \\
& +\frac{\gamma_{1 n} h-\sin \left(\gamma_{1 n} h\right) \cos \left(\gamma_{1 n} h\right)}{\gamma_{1 n} h-\sin \left(\gamma_{1 n} h\right) \cos \left(\gamma_{1 n} h\right)-\varepsilon^{2} \tan \left(\gamma_{1 n} h\right) \sin ^{2}\left(\gamma_{1 n} h\right)} \Delta k_{1}^{2} \\
& +\frac{-\varepsilon^{2} \tan \left(\gamma_{1 n} h\right) \sin ^{2}\left(\gamma_{1 n} h\right)}{\gamma_{1 n} h-\sin \left(\gamma_{1 n} h\right) \cos \left(\gamma_{1 n} h\right)-\varepsilon^{2} \tan \left(\gamma_{1 n} h\right) \sin ^{2}\left(\gamma_{1 n} h\right)} \Delta k_{2}^{2} .
\end{aligned}
$$

This resulting form reveals the dependence of $\Delta \kappa_{n}$ on $\Delta h, \Delta k_{1}$, and $\Delta k_{2}$.

\subsubsection{Derivation of $\Delta \kappa_{n}$ by using the perturbation equation (Eq.(5-24))}

In this section, we demonstrate that we can derive the same form as Eq.(5-40) by utilizing Eq.(5-24) obtained in Section 5.2.

The range-dependent Pekeris model corresponds to the case $L=2, M=2, \rho_{w}=\rho_{1}$, and $h_{2}=h(r)$ in our shallow water model in Figure 5-1 except that $g_{M}=0$. As discussed in the beginning of this section, however, Eq.(5-24) is applicable to the Pekeris waveguide as long as we deal with trapped modes only.

By using Eq.(5-28) through Eq.(5-32), we can express $\left.\gamma_{n}^{2}\right|_{n_{2} \pm 0},\left.u_{n}^{2}\right|_{h_{2}}$, and $\left.\left(\partial u_{n} / \partial z\right)^{2}\right|_{n_{2}}$ as follows: 


$$
\begin{aligned}
& \left.\gamma_{n}^{2}\right|_{h_{2}+0}=\gamma_{1 n}^{2} \equiv k_{1}^{2}-\kappa_{n}^{2} \\
& \left.\gamma_{n}^{2}\right|_{n_{n}-0}=\gamma_{2 n}^{2} \equiv k_{2}^{2}-\kappa_{n}^{2} \\
& \left.u_{n}^{2}\right|_{n}=\left(\frac{2 \rho_{1} \gamma_{1 n}}{\gamma_{1 n} h-\sin \left(\gamma_{1 n} h\right) \cos \left(\gamma_{1 n} h\right)-\varepsilon^{2} \tan \left(\gamma_{1 n} h\right) \sin ^{2}\left(\gamma_{1 n} h\right)}\right) \sin ^{2}\left(\gamma_{1 n} h\right) \\
& \left.\left(\frac{1}{\rho} \frac{\partial u_{n}}{\partial z}\right)^{2}\right|_{h_{2}}=\frac{\gamma_{1 n}^{2}}{\rho_{1}}\left(\frac{2 \gamma_{1 n}}{\gamma_{1 n} h-\sin \left(\gamma_{1 n} h\right) \cos \left(\gamma_{1 n} h\right)-\varepsilon^{2} \tan \left(\gamma_{1 n} h\right) \sin ^{2}\left(\gamma_{1 n} h\right)}\right) \cos ^{2}\left(\gamma_{1 n} h\right)
\end{aligned}
$$

Putting Eqs.(5-41)-(5-44) into the first term in Eq.(5-24) yields

$$
\begin{aligned}
\sum_{j=2}^{2}\left[\left.\left(\frac{\left.\gamma_{n}^{2}\right|_{h_{j}-0}}{\rho_{j-1}}-\frac{\gamma_{n}^{2} \mid h_{j}+0}{\rho_{j}}\right) u_{n}^{2}\right|_{h_{j}}+\left.\left(\rho_{j-1}-\rho_{j}\right)\left(\frac{1}{\rho} \frac{\partial u_{n}}{\partial z}\right)^{2}\right|_{h_{j}}\right] \Delta h_{j} \\
=\left[\left(\frac{\gamma_{1 n}^{2}}{\rho_{1}}-\frac{\gamma_{2 n}^{2}}{\rho_{2}}\right) \rho_{1} \sin ^{2}\left(\gamma_{1 n} h\right)+\left(\rho_{1}-\rho_{2}\right) \frac{\gamma_{1 n}^{2}}{\rho_{1}} \cos ^{2}\left(\gamma_{1 n} h\right)\right] \\
\\
\quad\left(\frac{2 \gamma_{1 n}}{\gamma_{1 n} h-\sin \left(\gamma_{1 n} h\right) \cos \left(\gamma_{1 n} h\right)-\varepsilon^{2} \tan \left(\gamma_{1 n} h\right) \sin ^{2}\left(\gamma_{1 n} h\right)}\right) \Delta h .
\end{aligned}
$$

The members in the bracket on the right hand side of Eq.(5-45) may be further rearranged by using Eq.(5-28) as

$$
\begin{aligned}
{[\quad] } & =\left[\left(\gamma_{1 n}^{2}-\varepsilon \gamma_{2 n}^{2}\right) \sin ^{2}\left(\gamma_{1 n} h\right)+\left(1-\frac{1}{\varepsilon}\right) \gamma_{1 n}^{2} \cos ^{2}\left(\gamma_{1 n} h\right)\right] \\
& =\left[\left(\gamma_{1 n}^{2}-\varepsilon \frac{\gamma_{1 n}^{2} \cot ^{2}\left(\gamma_{1 n} h\right)}{-\varepsilon^{2}}\right) \sin ^{2}\left(\gamma_{1 n} h\right)+\left(1-\frac{1}{\varepsilon}\right) \gamma_{1 n}^{2} \cos ^{2}\left(\gamma_{1 n} h\right)\right] \\
& =\gamma_{1 n}^{2} .
\end{aligned}
$$


Thus the first term in Eq.(5-24) finally becomes

$$
\begin{gathered}
\sum_{j=2}^{2}\left[\left.\left(\frac{\left.\gamma_{n}^{2}\right|_{h_{j}-0}}{\rho_{j-1}}-\frac{\left.\gamma_{n}^{2}\right|_{h_{j}+0}}{\rho_{j}}\right) u_{n}^{2}\right|_{h_{j}}+\left.\left(\rho_{j-1}-\rho_{j}\right)\left(\frac{1}{\rho} \frac{\partial u_{n}}{\partial z}\right)^{2}\right|_{h_{j}}\right] \Delta h_{j} \\
=\frac{2 \gamma_{1 n}^{3}}{\gamma_{1 n} h-\sin \left(\gamma_{1 n} h\right) \cos \left(\gamma_{1 n} h\right)-\varepsilon^{2} \tan \left(\gamma_{1 n} h\right) \sin ^{2}\left(\gamma_{1 n} h\right)} \Delta h .
\end{gathered}
$$

In the same way, the second term in Eq. $(5-24)$ becomes

$$
\begin{aligned}
& \frac{1}{\rho_{w}} \int_{0}^{h_{n}} \Delta k^{2} u_{n}^{2} d z \\
& \quad=\frac{\Delta k_{1}^{2}}{\rho_{1}} \frac{2 \rho_{1} \gamma_{1 n}}{\gamma_{1 n} h-\sin \left(\gamma_{1 n} h\right) \cos \left(\gamma_{1 n} h\right)-\varepsilon^{2} \tan \left(\gamma_{1 n} h\right) \sin ^{2}\left(\gamma_{1 n} h\right)} \int_{0}^{h} \sin ^{2}\left(\gamma_{1 n} z\right) d z \\
& =\frac{\gamma_{1 n} h-\sin \left(\gamma_{1 n} h\right) \cos \left(\gamma_{1 n} h\right)}{\gamma_{1 n} h-\sin \left(\gamma_{1 n} h\right) \cos \left(\gamma_{1 n} h\right)-\varepsilon^{2} \tan \left(\gamma_{1 n} h\right) \sin ^{2}\left(\gamma_{1 n} h\right)} \Delta k_{1}^{2} .
\end{aligned}
$$

Similarly the third term in Eq. (5-24) becomes

$$
\begin{aligned}
& \sum_{j=2}^{2} \frac{1}{\rho_{j}} \int_{h_{j}}^{h_{j+1}} \Delta k^{2} u_{n}^{2} d z \\
& \quad=\frac{\Delta k_{2}^{2}}{\rho_{2}} \frac{2 \rho_{1} \gamma_{1 n}}{\gamma_{1 n} h-\sin \left(\gamma_{1 n} h\right) \cos \left(\gamma_{1 n} h\right)-\varepsilon^{2} \tan \left(\gamma_{1 n} h\right) \sin ^{2}\left(\gamma_{1 n} h\right)} \sin ^{2}\left(\gamma_{1 n} h\right) \int_{h}^{\infty} e^{i 2 \gamma_{2 n}(z-k)} d z \\
& =\frac{2 \varepsilon \gamma_{1 n}}{\gamma_{1 n} h-\sin \left(\gamma_{1 n} h\right) \cos \left(\gamma_{1 n} h\right)-\varepsilon^{2} \tan \left(\gamma_{1 n} h\right) \sin ^{2}\left(\gamma_{1 n} h\right)} \frac{\sin ^{2}\left(\gamma_{1 n} h\right)}{-2 i \gamma_{2 n}} \Delta k_{2}^{2} \\
& =\frac{-\varepsilon^{2} \tan \left(\gamma_{1 n} h\right) \sin ^{2}\left(\gamma_{1 n} h\right)}{\gamma_{1 n} h-\sin \left(\gamma_{1 n} h\right) \cos \left(\gamma_{1 n} h\right)-\varepsilon^{2} \tan \left(\gamma_{1 n} h\right) \sin ^{2}\left(\gamma_{1 n} h\right)} \Delta k_{2}^{2}
\end{aligned}
$$

Subsequently, the three results in Eqs.(5-47), (5-48), and (5-49) are added in accordance with Eq.(5-24), yielding 


$$
\begin{aligned}
\Delta \kappa_{n}^{2}= & \frac{2 \gamma_{1 n}^{3}}{\gamma_{1 n} h-\sin \left(\gamma_{1 n} h\right) \cos \left(\gamma_{1 n} h\right)-\varepsilon^{2} \tan \left(\gamma_{1 n} h\right) \sin ^{2}\left(\gamma_{1 n} h\right)} \Delta h \\
& +\frac{\gamma_{1 n} h-\sin \left(\gamma_{1 n} h\right) \cos \left(\gamma_{1 n} h\right)}{\gamma_{1 n} h-\sin \left(\gamma_{1 n} h\right) \cos \left(\gamma_{1 n} h\right)-\varepsilon^{2} \tan \left(\gamma_{1 n} h\right) \sin ^{2}\left(\gamma_{1 n} h\right)} \Delta k_{1}^{2} \\
& +\frac{-\varepsilon^{2} \tan \left(\gamma_{1 n} h\right) \sin ^{2}\left(\gamma_{1 n} h\right)}{\gamma_{1 n} h-\sin \left(\gamma_{1 n} h\right) \cos \left(\gamma_{1 n} h\right)-\varepsilon^{2} \tan \left(\gamma_{1 n} h\right) \sin ^{2}\left(\gamma_{1 n} h\right)} \Delta k_{2}^{2} .
\end{aligned}
$$

Therefore it is immediately seen that Eq.(5-50) is completely equivalent to Eq.(5-40), which we obtained independently in Section 5.3.2.

In this section, we demonstrated that the perturbation equation (Eq.(5-24)) derived in Section 5.2 provides an accurate result when applied to the range-dependent Pekeris waveguide. In this simple waveguide, we represented the range variation of the local eigenvalues $\Delta \kappa_{n}$ explicitly in terms of $\Delta h, \Delta k_{1}$, and $\Delta k_{2}$.

In the next section, by representing the sound speed profile in our multilayered model with the use of the $n^{2}$-linear profile, we will express $\Delta \kappa_{n}$ explicitly in terms of the perturbed geoacoustic parameters.

\subsection{Range-dependent $\boldsymbol{n}^{2}$-linear profile}

Section 5.2 showed how the range variation of the local eigenvalues depends on the range variations of both the interface depth and the wavenumber in each sediment layer. But, as seen from Eq.(5-24), the resulting equation includes integrals having the range variation of the wavenumber inside the integrals. In order to express the range variations explicitly, we need to execute the integrals in Eq.(5-24) by specifying the sound speed profile in each sediment layer. Here we use the $n^{2}$-linear profile to represent the sound speed profiles. 


\subsubsection{Definition of a range-dependent $\boldsymbol{n}^{2}$-linear profile}

In this subsection, we will approximate the sound speed profile in each sediment layer by using the $n^{2}$-linear curve with range-dependent coefficients, which are defined by

$$
c(r, z)=c_{j}(r)\left[1-\frac{2 g_{j}(r)}{c_{j}(r)}\left(z-\bar{h}_{j}\right)\right]^{-\frac{1}{2}} \quad\left(h_{j}(r) \leq z \leq h_{j+1}(r)\right),
$$

where $c_{j}(r) \equiv c\left(r, \bar{h}_{j}\right)$ is the sound speed at $z=\bar{h}_{j}, g_{j}(r)$ is the sound speed gradient in the $j$ th layer, and $\bar{h}_{j} \equiv h_{j}\left(r_{0}\right)$ stands for the sediment interface depth at some reference range point $r_{0}$ (e.g., $r_{0}=0$ ). Note that $\bar{h}_{j}$ is a constant (see Figure 5-3). Due to the relation

$$
\left|g_{j}(r)\right|<<c_{j}(r)
$$

Eq.(5-51) can be related approximately to the following linear profile:

$$
c(r, z) \approx c_{j}(r)+g_{j}(r)\left(z-\bar{h}_{j}\right) \quad\left(h_{j}(r) \leq z \leq h_{j+1}(r)\right) .
$$

Thus we see that the difference in the sound speeds represented by Eqs.(5-51) and (5-53) increases with increasing $\left|z-\bar{h}_{j}\right|$. It should be noted that $g_{j}(r)$ can take either a positive or negative value except for the lowest layer $l=M$, where only a positive gradient is allowed to meet the boundary condition given in Eq.(5-4).

To accommodate attenuation, Eq.(5-51) may be extended to the following form of the wavenumber:

$$
k^{2}(r, z)=K_{j}^{2}(r)+\mu_{j}(r)\left(z-\bar{h}_{j}\right) \quad\left(h_{j}(r) \leq z \leq h_{j+1}(r)\right),
$$

with

$$
K_{j}(r) \equiv k_{j}(r)+i \alpha_{j}: \text { complex wavenumber at } z=\bar{h}_{j} \text {, }
$$



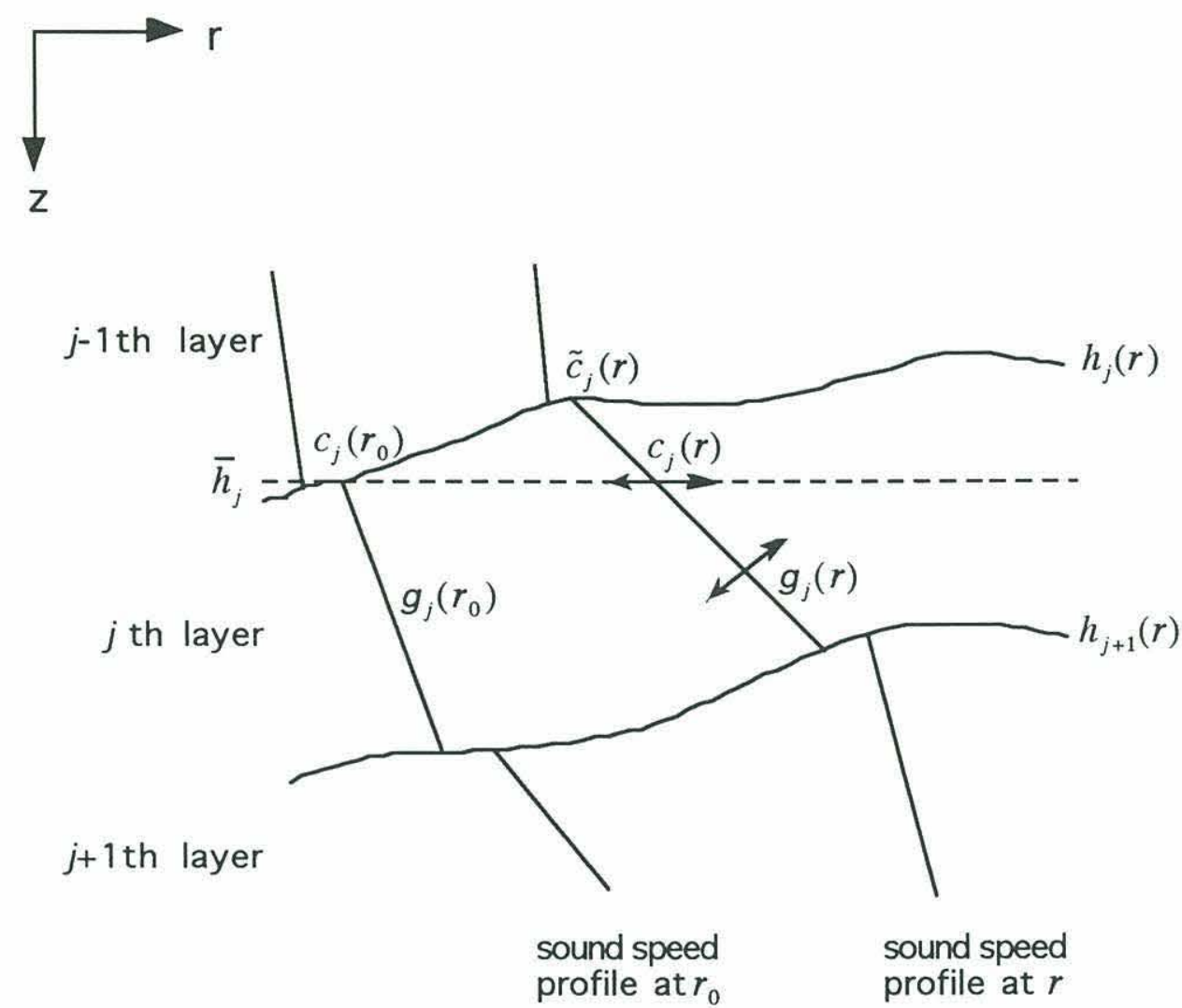

Figure 5-3: Range-dependent $n^{2}$-linear profile. 


$$
\mu_{j}(r) \equiv-\frac{2 g_{j}(r) k_{j}^{2}(r)}{c_{j}(r)}: \text { gradient of } k^{2} \text { in the } j \text { th layer, }
$$

where $k_{j}(r) \equiv \frac{\omega}{c_{j}(r)}$ is the real part of the wavenumber at $\mathrm{z}=\bar{h}_{j}$ and $\alpha_{j}$ is the attenuation coefficient in the $j$ th layer.

From Eq.(5-54) it is immediately seen that the lateral variations of $k^{2}$ and the rangedependent parameters are related as

$$
\Delta k^{2}(r, z)=2 K_{j}(r) \Delta k_{j}(r)+\left(z-\bar{h}_{j}\right) \Delta \mu_{j}(r) \quad\left(h_{j}(r) \leq z \leq h_{j+1}(r)\right),
$$

where $\Delta K_{j}^{2}=2 K_{j} \Delta k_{j}$ has been used since $\alpha_{j}$ is assumed to be range-independent. In addition, $\Delta k_{j}$ and $\Delta \mu_{j}$ are uniquely related to $\Delta c_{j}$ and $\Delta g_{j}$ as

$$
\left[\begin{array}{l}
\Delta k_{j} \\
\Delta \mu_{j}
\end{array}\right]=\left[\begin{array}{cc}
-\frac{\omega}{c_{j}^{2}} & 0 \\
\frac{6 \omega^{2} g_{j}}{c_{j}^{4}} & -\frac{2 \omega^{2}}{c_{j}^{3}}
\end{array}\right]\left[\begin{array}{l}
\Delta c_{j} \\
\Delta g_{j}
\end{array}\right] .
$$

Since $\frac{6 \omega^{2}\left|g_{j}\right|}{c_{j}^{4}} \ll<\frac{2 \omega^{2}}{c_{j}^{3}}$ due to Eq.(5-52), the contribution of the $\Delta c_{j}$-term to $\Delta \mu_{j}$ is substantially smaller than the contribution of the $\Delta g_{j}$-term. Hence $\Delta \mu_{j}$ is nearly proportional to $\Delta g_{j}$; but when $\Delta g_{j} \approx 0$, then the $\Delta c_{j}$-term cannot be ignored. Conversely $\Delta k_{j}$ is simply proportional to $\Delta c_{j}$.

Here it would be of interest to compare Eq.(5-54) with another representation given by

$$
k^{2}(r, z)=\tilde{K}_{j}^{2}(r)+\mu_{j}(r)\left[z-h_{j}(r)\right] \quad\left(h_{j}(r) \leq z \leq h_{j+1}(r)\right),
$$


where $\tilde{K}_{j}(r) \equiv \tilde{k}_{j}(r)+i \alpha_{j}$ with $\tilde{k}_{j}(r) \equiv \frac{\omega}{\tilde{c}_{j}(r)}$ and $\tilde{c}_{j}(r) \equiv c\left(r, h_{j}(r)\right)$, which stands for a sound speed at the interface depth $h_{j}(r)$. Thus the range dependence of $\tilde{k}_{j}(r)$ and $\tilde{c}_{j}(r)$ comes from the variations of both the medium itself and the interface depth. This can easily be checked using the following identity:

$$
\tilde{K}_{j}^{2}(r)=K_{j}^{2}(r)+\mu_{j}(r)\left[h_{j}(r)-\bar{h}_{j}\right],
$$

which can be obtained by comparing Eq.(5-54) and Eq.(5-59) rewritten as

$$
k^{2}(r, z)=\left\{\tilde{K}_{j}^{2}(r)-\mu_{j}(r)\left[h_{j}(r)-\bar{h}_{j}\right]\right\}+\mu_{j}(r)\left(z-\bar{h}_{j}\right) .
$$

Recalling that $K_{j}(r)$ represents the wavenumber at the fixed depth $\bar{h}_{j}$, it is seen that $\tilde{K}_{j}(r)$ includes the effect of the sediment interface variation in the second term on the right-hand side of Eq.(5-60). Therefore Eq.(5-54) is more convenient than Eq.(5-59) in representing the range variation of the wavenumber.

In order to understand the role of the parameters in Eq.(5-54) further, let us consider the special case below. If the medium in some layer is range independent, i.e., $\Delta k_{j}(r)=0$ and $\Delta \mu_{j}(r)=0$, but has the layer depth variation $\Delta h_{j}(r) \neq 0$, then we have $2 \tilde{K}_{j}(r) \Delta \tilde{k}_{j}(r)=\mu_{j}(r) \Delta h_{j}(r)$ from Eq.(5-60); the wavenumber $\tilde{k}_{j}(r)$ at the sediment interface varies in accordance with the depth variation of the interface itself. Let us refer to this as Case A. On the contrary, if we keep $\tilde{k}_{j}(r)$ constant (range-independent) at $h_{j}(r)$, i.e., $\Delta \tilde{k}_{j}(r)=0$, then the medium in this layer has to be range-dependent and varies with range so as to satisfy $2 K_{j}(r) \Delta k_{j}(r)+\left[h_{j}(r)-\bar{h}_{j}\right] \Delta \mu_{j}(r)=-\mu_{j}(r) \Delta h_{j}(r)$. This is Case B; the medium and layer interface vary so that two kinds of range variations cancel out each other at $h_{j}(r)$.

Now, assume a simple waveguide consisting of a single-layer bottom and a water column with constant sound speed as shown in Figure 5-4. Case B, then, corresponds to 


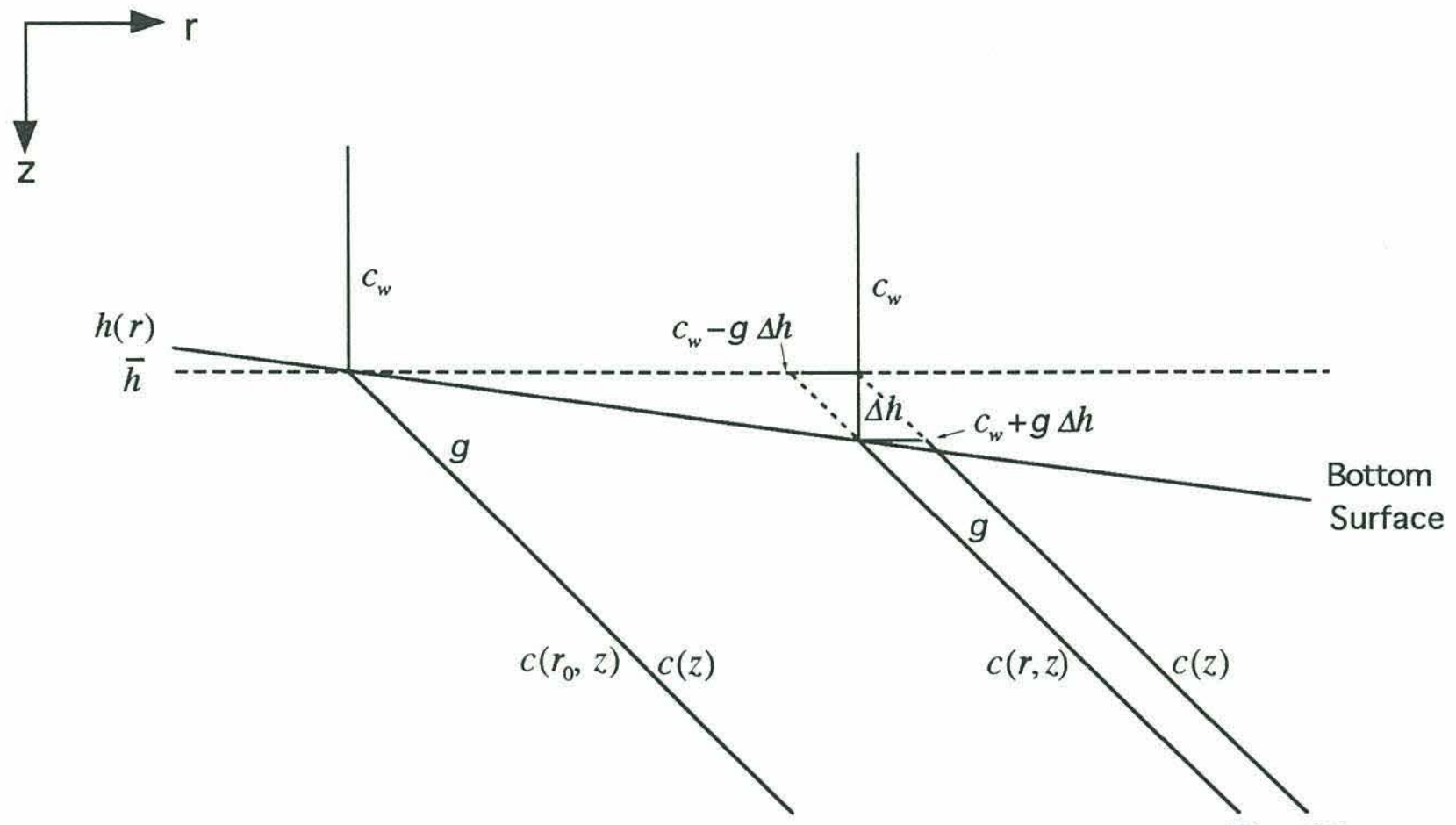

(B) (A)

sound speed

sound speed

profile at $r_{0}$

profile at $r$

Figure 5-4: Single sediment layer with constant sound speed gradient. 
the waveguide having a range-independent plane-wave reflection coefficient, although the acoustic properties of this bottom medium vary with range. In contrast, for Case A, the reflection coefficient varies with range due to the bathymetric change, irrespective of the range-independent properties of the bottom medium itself.

\subsubsection{Evaluation of integrals in the perturbation equation (Eq.(5-24))}

In this subsection, let us evaluate the integrals in Eq.(5-24) by employing the rangedependent $n^{2}$-linear profile in Eq.(5-54). To do this, we first represent the local eigenfunction in terms of the parameters of this profile.

It is well known that, given the $n^{2}$-linear profile, the eigenfunctions $u_{n}$ can be expressed in terms of the Airy functions $A i$ and $B i[54,62,63]$. Namely, by inserting Eq.(5-54) in place of $k^{2}(r, z)$ in Eq.(5-1), this equation is converted to the following Stokes equation:

$$
\frac{\partial^{2} u_{n}}{\partial \xi_{j}^{2}}+\xi_{j} u_{n}=0 \quad\left(h_{j}(r) \leq z \leq h_{j+1}(r)\right)
$$

with

$$
\begin{aligned}
\xi_{j}(r, z) & =\frac{\gamma_{n}^{2}}{\eta_{j}^{2}}=\frac{1}{\eta_{j}^{2}}\left(k^{2}-\kappa_{n}^{2}\right) \\
& =\frac{1}{\eta_{j}^{2}}\left(K_{j}^{2}-\kappa_{n}^{2}\right)+\eta_{j}\left(z-\bar{h}_{j}\right)
\end{aligned}
$$

and

$$
\eta_{j}(r)=\mu_{j}^{\dagger}
$$

where $\gamma_{n}(r, z)$ is the vertical wavenumber. Since $A i(-\xi)$ and $B i(-\xi)$ are independent solutions satisfying Eq.(5-62), $u_{n}$ can be represented by

$$
u_{n}(r, z)=C_{j 1} A i\left(-\xi_{j}\right)+C_{j 2} B i\left(-\xi_{j}\right) \quad\left(h_{j}(r) \leq z \leq h_{j+1}(r)\right),
$$


where $C_{j l}$ and $C_{j 2}$ are the range-dependent coefficients determined by the boundary conditions in Eqs.(5-3) and (5-4) and the normalization condition in Eq.(5-2).

Now let us substitute Eq.(5-57) into Eq.(5-24) and evaluate the integrals in Eq.(524). Each integral is then split into two integrals:

$$
\int_{h_{j}}^{h_{j+1}} u_{n}^{2} \Delta k^{2} d z=2 K_{j} \Delta k_{j} \int_{h_{j}}^{h_{j+1}} u_{n}^{2} d z+\Delta \mu_{j} \int_{h_{j}}^{h_{j+1}} u_{n}^{2}\left(z-\bar{h}_{j}\right) d z
$$

By changing the variable $z$ to $\xi$ in each integral on the right-hand side of Eq.(5-67), it follows that

$$
\int_{h_{j}}^{h_{j+1}} u_{n}^{2} d z=\frac{1}{\eta_{j}} \int_{\xi_{j}\left(h_{j}\right)}^{\xi_{j}\left(h_{j+1}\right)} u_{n}^{2} d \xi_{j}
$$

and

$$
\int_{h_{j}}^{h_{j+1}} u_{n}^{2}\left(z-\bar{h}_{j}\right) d z=\frac{1}{\eta_{j}^{2}} \int_{\xi_{j}\left(h_{j}\right)}^{\xi_{j}\left(h_{j+1}\right)} u_{n}^{2} \xi_{j} d \xi_{j}-\frac{K_{j}^{2}-\kappa_{n}^{2}}{\eta_{j}^{4}} \int_{\xi_{j}\left(h_{j}\right)}^{\xi_{j}\left(h_{j+1}\right)} u_{n}^{2} d \xi_{j}
$$

Each integral on the right-hand side of both Eq.(5-68) and Eq.(5-69) can then be evaluated in closed form by virtue of the following identity for the Airy function:

$$
\int \Phi^{2} d \xi=\xi \Phi^{2}+\Phi^{\prime 2}
$$

and

$$
\int \xi \Phi^{2} d \xi=\frac{1}{3}\left(\xi^{2} \Phi^{2}+\xi \Phi^{\prime 2}-\Phi \Phi^{\prime}\right)
$$

where $\Phi(\xi)$ is the Airy function or a linear combination of the solutions of Eq.(5-62), and the prime denotes the derivative $\frac{d}{d \xi}$. Hence, we employ Eq.(5-70) to evaluate Eq.(568) and then use of the relation $\xi_{j}=\gamma_{n}^{2} / \eta_{j}^{2}$ given by Eq.(5-63) to obtain 


$$
\int_{h_{j}}^{h_{j+1}} u_{n}^{2} d z=\left.\frac{1}{\eta_{j}}\left[\frac{\gamma_{n}^{2}}{\eta_{j}^{2}} u_{n}^{2}+\left(\frac{1}{\eta_{j}} \frac{\partial u_{n}}{\partial z}\right)^{2}\right]\right|_{h_{j}} ^{h_{j+1}}
$$

In Eq.(5-72), use of the relations $\mu_{j}=\eta_{j}^{3}$ given by Eq.(5-65) and $\frac{d}{d \xi_{j}}=\frac{1}{\eta_{j}} \frac{\partial}{\partial z}$ given by Eq.(5-64) yields

$$
\int_{h_{j}}^{h_{j+1}} u_{n}^{2} d z=\frac{1}{\mu_{j}}\left[\gamma_{n}^{2} u_{n}^{2}+\left(\frac{\partial u_{n}}{\partial z}\right)^{2}\right]_{h_{j}}^{h_{j+1}}
$$

In the same way, we can evaluate the first integral on the right-hand side of Eq.(5-69); we can also evaluate the second integral by using Eq.(5-71) as

$$
\begin{aligned}
& \int_{h_{j}}^{h_{j+1}} u_{n}^{2}\left(z-\bar{h}_{j}\right) d z=\left.\frac{1}{3 \eta_{j}^{2}}\left[\left(\frac{\gamma_{n}^{2}}{\eta_{j}^{2}}\right)^{2} u_{n}^{2}+\frac{\gamma_{n}^{2}}{\eta_{j}^{2}}\left(\frac{1}{\eta_{j}} \frac{\partial u_{n}}{\partial z}\right)^{2}-u_{n}\left(\frac{1}{\eta_{j}} \frac{\partial u_{n}}{\partial z}\right)\right]\right|_{h_{j}} ^{h_{j+1}} \\
&-\left.\frac{K_{j}^{2}-\kappa_{n}^{2}}{\eta_{j}^{4}}\left[\frac{\gamma_{n}^{2}}{\eta_{j}^{2}} u_{n}^{2}+\left(\frac{1}{\eta_{j}} \frac{\partial u_{n}}{\partial z}\right)^{2}\right]\right|_{h_{j}} ^{h_{j+1}} \\
&=\left.\frac{1}{3 \mu_{j}^{2}}\left[\left(\gamma_{n}^{2}-3 \bar{\gamma}_{n}^{2}\right)\left(\gamma_{n}^{2} u_{n}^{2}+\left(\frac{\partial u_{n}}{\partial z}\right)^{2}\right)-\mu_{j} u_{n} \frac{\partial u_{n}}{\partial z}\right]\right|_{h_{j}} ^{h_{j+1}},
\end{aligned}
$$

where $\bar{\gamma}_{n} \equiv \gamma_{n}\left(r, \bar{h}_{j}\right)$, i.e., $\bar{\gamma}_{n}^{2} \equiv K_{j}^{2}-\kappa_{n}^{2}$ has been employed in the step from Eq.(5-74a) to Eq.(5-74b). As a result of the substitution of both Eq.(5-73) and Eq.(5-74b) into Eq.(5$67)$, we can finally obtain

$$
\int_{h_{j}}^{h_{j+1}} u_{n}^{2} \Delta k^{2} d z=\frac{1}{\mu_{j}}\left[2 K_{j} \Delta k_{j}+\frac{\Delta \mu_{j}}{3 \mu_{j}}\left(\gamma_{n}^{2}-3 \bar{\gamma}_{n}^{2}\right)\right]\left(\gamma_{n}^{2} u_{n}^{2}+\left(\frac{\partial u_{n}}{\partial z}\right)^{2}\right)_{h_{j}}^{h_{j_{j+1}}}-\left.\frac{\Delta \mu_{j}}{3 \mu_{j}} u_{n} \frac{\partial u_{n}}{\partial z}\right|_{h_{j}} ^{h_{j+1}} .
$$


Further, if we make use of the relation given by Eq.(5-58), then Eq.(5-75) can be rewritten in terms of $\Delta c_{j}$ and $\Delta g_{j}$ instead of $\Delta k_{j}$ and $\Delta \mu_{j}$.

Thus, by substituting Eq.(5-75) into Eq.(5-24), the range-dependent variation of the local eigenvalue can be expressed as

$$
\begin{aligned}
\Delta \kappa_{n}^{2}= & \sum_{j=L}^{M}\left[\left.\left(\frac{\left.\gamma_{n}^{2}\right|_{h_{j}-0}}{\rho_{j-1}}-\frac{\left.\gamma_{n}^{2}\right|_{h_{j}+0}}{\rho_{j}}\right) u_{n}^{2}\right|_{h_{j}}+\left.\left(\rho_{j-1}-\rho_{j}\right)\left(\frac{1}{\rho} \frac{\partial u_{n}}{\partial z}\right)^{2}\right|_{h_{j}}\right] \Delta h_{j}+\frac{1}{\rho_{w}} \int_{0}^{h_{h}} \Delta k^{2} u_{n}^{2} d z \\
& +\sum_{j=L}^{M} \frac{1}{\rho_{j} \mu_{j}}\left(\gamma_{n}^{2} u_{n}^{2}+\left(\frac{\partial u_{n}}{\partial z}\right)^{2}\right)_{h_{j}}^{h_{j+1}} 2 K_{j} \Delta k_{j} \\
& +\sum_{j=L}^{M} \frac{1}{3 \rho_{j} \mu_{j}}\left[\frac{\gamma_{n}^{2}-3 \bar{\gamma}_{n}^{2}}{\mu_{j}}\left(\gamma_{n}^{2} u_{n}^{2}+\left(\frac{\partial u_{n}}{\partial z}\right)^{2}\right)_{h_{j}}^{h_{j+1}}-\left.u_{n} \frac{\partial u_{n}}{\partial z}\right|_{h_{j}} ^{h_{j+1}}\right] \Delta \mu_{j}
\end{aligned}
$$

If we approximate the profile in each layer including the water layer by a sequence of $n^{2}$-linear curves, then each integral in Eq.(5-76) can be evaluated and expressed in the same way. It should be noted, however, that the sound speed in the water column is continuous across layer interfaces, so that $k_{j}$ and $\mu_{j}$ cannot be chosen independently of each other.

If the sound speed profile in some sediment layer cannot be approximated by a single $n^{2}$-linear curve, then we can divide this layer into sublayers with single sound speed gradients. This treatment is often required for modeling sand layers, because the sound speed gradient of sand decreases rapidly with depth [84], and thus a single $n^{2}$-linear curve cannot fit this profile. Again attention has to be paid to the dependence of $k_{j}$ and $\mu_{j}$ on each other for the same reason as above; the sound speed has to be continuous across the various sublayers.

By using an $n^{2}$-linear curve for the sound speed profile in each sediment layer, we showed that the range variation of the local eigenvalue can be related to the range variation of the geoacoustic parameters in each layer in closed form. We can make use of 
this perturbed relation (Eq.(5-76)) to analyze how the local eigenvalues are affected by the range-varying sediment structure and geoacoustic parameters in the current model. Conversely this equation can be used in an inverse method to determine the sediment properties from the local eigenvalues, a topic which will be pursued in the next section.

\subsection{Inversion method to determine range-dependent geoacoustic parameters}

In this section, let us assume that we do not know the range-dependent geoacoustic parameters in our shallow water model except for those near $r=0$. Local eigenvalues, however, are known as a result of applying the Hankel transform with a sliding window to the measured pressure field as shown in Chapters 3 and 4. The objective here is to determine these unknown geoacoustic parameters by inverting the perturbation relation given by Eq.(5-76). We use the local eigenvalues as input data in this equation.

\subsubsection{Formulation of the inverse problem}

In the first place, we need to formulate an equation for the unknown geoacoustic parameters in our shallow water model. Suppose that we know the local eigenvalues of $N$ modes. If the bottom environment can be modeled as described in the previous sections, then each eigenvalue varies with range so as to satisfy Eq.(5-76) and, consequently, the set of these equations for $N$ modes forms a set of simultaneous equations, which can be rewritten in vector form:

$$
\Delta \kappa_{n}=\Delta v_{n}+\mathbf{H}_{n j} \Delta h_{j}+\mathbf{S}_{n j} \Delta k_{j}+\mathrm{T}_{n j} \Delta \mu_{j},
$$

where $\mathrm{H}, \mathrm{S}$ and $\mathrm{T}$ are $N \times(M-L+1)$ matrices defined by

$$
\mathrm{H}_{n j}=\frac{1}{2 \kappa_{n}}\left[\left.\left(\frac{\left.\gamma_{n}^{2}\right|_{h_{j}-0}}{\rho_{j-1}}-\frac{\left.\gamma_{n}^{2}\right|_{h_{j}+0}}{\rho_{j}}\right) u_{n}^{2}\right|_{h_{j}}+\left.\left(\rho_{j-1}-\rho_{j}\right)\left(\frac{1}{\rho} \frac{\partial u_{n}}{\partial z}\right)^{2}\right|_{h_{j}}\right],
$$




$$
\begin{aligned}
& \mathrm{S}_{n j}=\left.\frac{K_{j}}{2 \kappa_{n} \rho_{j} \mu_{j}}\left(\gamma_{n}^{2} u_{n}^{2}+\left(\frac{\partial u_{n}}{\partial z}\right)^{2}\right)\right|_{h_{j}} ^{k_{j+1}}, \\
& \mathrm{~T}_{n j}=\left.\frac{1}{6 \kappa_{n} \rho_{j} \mu_{j}}\left[\frac{\gamma_{n}^{2}-3 \bar{\gamma}_{n}^{2}}{\mu_{j}}\left(\gamma_{n}^{2} u_{n}^{2}+\left(\frac{\partial u_{n}}{\partial z}\right)^{2}\right)-u_{n} \frac{\partial u_{n}}{\partial z}\right]\right|_{h_{j}} ^{k_{j+1}} .
\end{aligned}
$$

Both $\Delta \kappa_{n} \equiv\left(\Delta \kappa_{n}\right)$ and

$$
\Delta v_{n} \equiv\left(\frac{1}{2 \kappa_{n} \rho_{w}} \int_{0}^{h_{t}} \Delta k^{2} u_{n}^{2} d z\right)
$$

are vectors with $N$ elements; and $\Delta h_{j} \equiv\left(\Delta h_{j}\right), \Delta k_{j} \equiv\left(\Delta k_{j}\right)$, and $\Delta \mu_{j} \equiv\left(\Delta \mu_{j}\right)$ are vectors with $M-L+1$ elements. Although $N$ is the total number of modes to be incorporated in the inversion, these modes are not necessarily in sequence. We could choose the modes which are most sensitive to the variation of the geoacoustic parameters to be determined, as long as those modes are measurable. Moreover, it is possible to utilize the modes of different frequencies for $\Delta \kappa_{n}$ in Eq.(5-77) at the same time, because the bottom parameters to be inferred are assumed to be independent of the acoustic frequency.

Here $\Delta \kappa_{n}$ are immediately provided by taking the difference between two local eigenvalues separated by $\Delta r$ :

$$
\Delta \kappa_{n}(r)=\operatorname{Re}\left[\kappa_{n}(r+\Delta r)\right]-\operatorname{Re}\left[\kappa_{n}(r)\right],
$$

where only the real part is taken, because the measurement of $\kappa_{n}(r)$ is limited to the real part in the method used in Chapters 3 and 4 . In order to obtain the imaginary part, we would have to measure the amplitude of the output spectrum of the Hankel transform [83]. This however is beyond the scope of the present research. Thus in implementing Eqs.(5-78)-(5-80), only the real part of the eigenvalues is taken into account. As a matter 
of fact, the effect of the imaginary part is negligibly small in this inversion process, since we deal with first order perturbation of the local eigenvalues in Eq.(5-77).

In addition, we can expect that the sound speed in the water column is generally measurable with range in shallow water, so that $\Delta k(r, z)\left(0 \leq z \leq h_{L}\right)$ in Eq. (5-81) is known in advance. As a consequence, $\Delta v_{n}$ can be dealt with as input data as long as the local eigenfunction $u_{n}(r)$ is provided. Therefore, we may rewrite Eq.(5-77) as

$$
\Delta \kappa_{n}-\Delta v_{n}=\left[\mathbf{H}_{n j}, \mathbf{S}_{n j}, \mathbf{T}_{n j}\right]\left[\begin{array}{c}
\Delta h_{j} \\
\Delta k_{j} \\
\Delta \mu_{j}
\end{array}\right],
$$

or, equivalently,

$$
y=\mathrm{D} x,
$$

where $y \equiv \Delta \kappa_{n}-\Delta v_{n}$ and $x \equiv\left[\begin{array}{c}\Delta h_{j} \\ \Delta \boldsymbol{k}_{j} \\ \Delta \mu_{j}\end{array}\right]$ are vectors with $N$ and $3(M-L+1)$ elements,

respectively; and $\mathbf{D} \equiv\left[\mathbf{H}_{n j}, \mathbf{S}_{n j}, \mathbf{T}_{n j}\right]$ is a $N \times 3(M-L+1)$ matrix. Hence $\boldsymbol{y}$ is a known factor given as input data whereas $x$ is an unknown factor to be determined.

\subsubsection{Solution of the inverse problem}

In order to obtain the range-dependent geoacoustic parameters $h_{j}(r), k_{j}(r)$, and $\mu_{j}(r)$, we have to solve Eq.(5-84) for $x$ at different ranges.

To begin with, let us assume that the bottom environment is known at some range point $r_{\ell}$ near $r=0$, that is, $h_{j}\left(r_{\ell}\right), k_{j}\left(r_{\ell}\right)$ and $\mu_{j}\left(r_{\ell}\right)$ are known; then, the local eigenfunctions $u_{n}\left(r_{\ell}\right)$ at this range can be determined by solving Eq.(5-1). Upon use of those parameters and eigenfunctions in Eqs.(5-79)-(5-81), each component of $\mathbf{H}, \mathbf{S}$, and $\mathbf{T}$ can be evaluated along with $\Delta v_{n}$ at the range $r_{\ell}$. 
If we have enough modes to satisfy $N=3(M-L+1)$, then Eq.(5-84) can simply be inverted, yielding

$$
x=\mathrm{D}^{-1}\left(\Delta \kappa_{n}-\Delta v_{n}\right) .
$$

By using this $x$, we can then determine the range-dependent geoacoustic parameters at a new range point $r_{\ell}+\Delta r_{\ell}$ as

$$
\begin{aligned}
& \boldsymbol{h}_{j}\left(r_{\ell}+\Delta r_{\ell}\right) \sim \boldsymbol{h}_{j}\left(r_{\ell}\right)+\Delta \boldsymbol{h}_{j}\left(r_{\ell}\right), \\
& \boldsymbol{k}_{j}\left(r_{\ell}+\Delta r_{\ell}\right) \sim \boldsymbol{k}_{j}\left(r_{\ell}\right)+\Delta \boldsymbol{k}_{j}\left(r_{\ell}\right), \\
& \mu_{j}\left(r_{\ell}+\Delta r_{\ell}\right) \sim \mu_{j}\left(r_{\ell}\right)+\Delta \mu_{j}\left(r_{\ell}\right) .
\end{aligned}
$$

These results are correct to the first order, since the acoustic properties are assumed to vary gradually with range.

The validity of the results in Eq.(5-86) may be confirmed by comparing the measured local eigenvalues at $r_{\ell}+\Delta r_{\ell}$, that is $\kappa_{n}\left(r_{\ell}+\Delta r_{\ell}\right)$, and the calculated ones determined by solving Eq.(5-1) with the new bottom environment given by Eq.(5-86). When they do not agree closely, then we can repeat the same process after reducing the range step $\Delta r_{\ell}$.

The next step is to use the geoacoustic parameters obtained above at $r_{\ell}+\Delta r_{\ell}$ as a background for determining the parameters at the following new range point. Namely, by setting $\ell \rightarrow \ell+1$, we can repeat the same process as above. Note that $\Delta r_{\ell+1}$ is not necessarily equivalent to $\Delta r_{\ell}$. In general, $\Delta r$ may be chosen so that the linearity of $\Delta \kappa_{n}(r)$ is satisfied, at least in an approximate sense. The determination of $\Delta r$ will be discussed again when dealing with a numerical example in the next section.

Thus, given the known geoacoustic parameters as initial values at some range, we can determine them successively by repeating the inversion process at different ranges. 


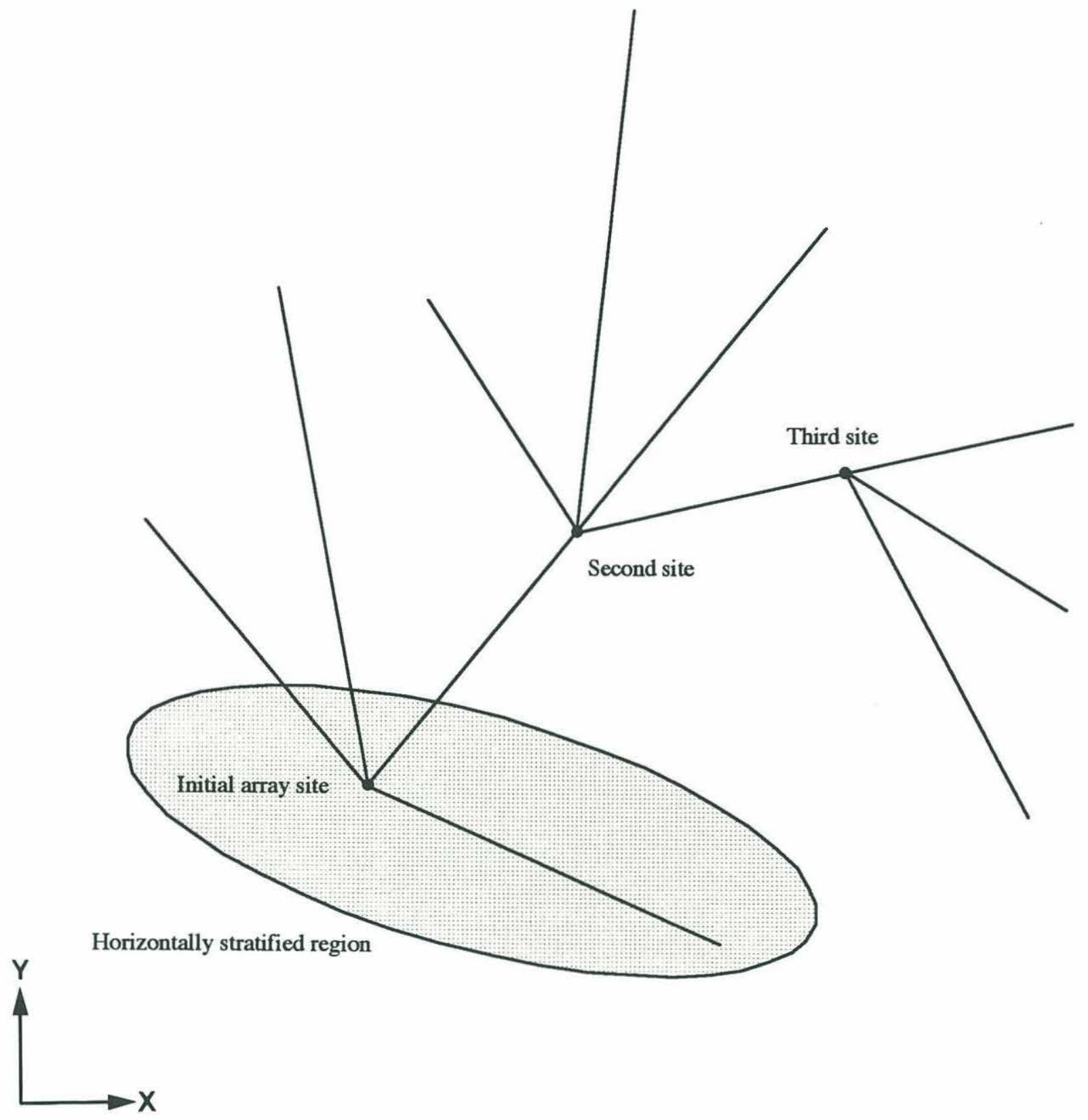

Figure 5-5: Extension of the inversion scheme from a horizontally stratified region to a range-dependent region. 
One of the issues arising from this inversion process is how to provide these initial parameters. We had a similar problem when using a mode filter that required the values of sediment properties at the site of the vertical array, as discussed in Chapter 3.3. As one of the ways of addressing this problem, we may make use of results obtained by other methods such as ones based on the travel times of broadband signals. For example, if we chose a locally, horizontally stratified region as an initial range point, the geoacoustic parameters in this region could be determined by resorting to inverse methods developed for the horizontally stratified case $[34,50]$; then we can employ the results obtained in this region as initial values for the current inverse scheme. Thus we can extend the inversion process from a locally, horizontally stratified region to a range-dependent region. Once this approach works well and the geoacoustic properties are determined along this extended range, then we can choose a new receiving point for the next array site from the points in this range, where the geoacoustic parameters for an initial value have been established (see Figure 5-5).

\subsubsection{Reducing the number of unknown parameters}

When deriving Eq.(5-85), we assumed that the total number of measurable modes is equal to the number of unknown geoacoustic parameters, but this cannot always be expected.

In general, the inverse problem for determining the geoacoustic parameters tends to be an underdetermined problem due to the limitations of real input data. Hence we must try to either increase the number of modes to be measured or decrease the number of unknown parameters to be determined. Concerning the former approach, we can employ the modes at different frequencies in the same simultaneous equations, Eq.(5-84), as mentioned in Section 5.5.2. However the maximum bottom depth which the current inversion method is capable of examining is automatically determined by the highest mode of the lowest frequency in use. In general, a mode is less sensitive to changes in 
the medium below its turning point depth; thus the experimental frequency imposes limits on the lowest sediment layer to be examined by the inversion process.

As for the latter approach of decreasing the number of unknown parameters, we can make use of information about the unknown parameters provided by other geophysical methods. For example, the interface depths of sediment layers could be inferred by using conventional seismic travel time analysis. The output data of these other methods do not have to be any of the parameters to be determined by our method, but can be information which relates to the desired unknown parameters. For instance, the travel time of broadband signals reflected or refracted from lower sediment layers serves to relate the sound speed profile and layer depth [43]. Or, if the sediment type of a particular layer is known along with other environmental information such as its depth, then the values taken by the geoacoustic parameters of that layer can be inferred within some range by referring to Hamilton's research results [84]. By using Lagrange multipliers [78], the information from the other methods can be combined with the inverse method considered presently.

Taking these methods into consideration, let us assume that some of the geoacoustic parameters are provided $a$ priori. Then we can shift those parameters, which are denoted by $\div$ below, from the right-hand side to the left-hand side in Eq.(5-83):

$$
\Delta \kappa_{n}-\Delta v_{n}-\left[\widehat{\mathbf{H}}_{n j}, \widehat{\mathbf{S}}_{n j}, \widehat{\mathbf{T}}_{n j}\right]\left[\begin{array}{c}
\Delta \widehat{\boldsymbol{h}}_{j} \\
\Delta \widehat{\boldsymbol{k}}_{j} \\
\Delta \widehat{\mu}_{j}
\end{array}\right]=\left[\breve{\mathbf{H}}_{n j}, \breve{\mathrm{S}}_{n j}, \breve{\mathrm{T}}_{n j}\right]\left[\begin{array}{c}
\Delta \breve{h}_{j} \\
\Delta \breve{k}_{j} \\
\Delta \breve{\mu}_{j}
\end{array}\right] .
$$

Rewriting the left-hand side as $\bar{y}$, and rewriting the unknown vector on the right-hand side as $\breve{\boldsymbol{x}}$, Eq.(5-87) leads to

$$
\breve{y}=\breve{\mathbf{D}} \breve{x} .
$$


Thus if the total number of these known parameters is $F$, then $\breve{x}$ becomes a [3(M-L+1)$F] \times 1$ vector and $\breve{\mathbf{D}}$ becomes a $N \times[3(M-L+1)-\mathrm{F}]$ matrix. For example, if the bathymetry is measured using an echo sounder and $h_{L}(r)$ is known, then the following amount can be subtracted from $\Delta \kappa_{n}$ as a part of $\widehat{\mathbf{H}}_{n j} \widehat{h}_{j}$ in Eq.(5-87):

$$
\mathrm{H}_{n L} \Delta h_{L}=\frac{\Delta h_{L}}{2 \kappa_{n}}\left[\left.\left(\frac{\left.\gamma_{n}^{2}\right|_{h_{L}-0}}{\rho_{w}}-\frac{\left.\gamma_{n}^{2}\right|_{h_{L}+0}}{\rho_{L}}\right) u_{n}^{2}\right|_{h_{L}}+\left.\left(\rho_{w}-\rho_{L}\right)\left(\frac{1}{\rho} \frac{\partial u_{n}}{\partial z}\right)^{2}\right|_{h_{L}}\right]
$$

where $\rho_{L-1}=\rho_{w}$ is set. If the sediment layer depths are determined using travel time analysis, then we could reduce the unknown parameters further by using Eq.(5-87).

\subsubsection{Solution of the underdetermined problem}

Even if we take advantage of the methods described in Section 5.5.3, when $N<[3(M-$ $L+1)-F]$, then unfortunately we have to solve for an underdetermined problem. An effective method of dealing with this problem is to make use of the SVD method [43,50], which was also utilized for the overdetermined problem in Chapter 3.3. This method can decompose the matrix $\breve{\mathbf{D}}$ as

$$
\breve{\mathbf{D}}=\mathbf{A}_{I} \Lambda_{I} \mathbf{B}_{I}^{t},
$$

where $\Lambda_{I}$ is an $I X I$ diagonal matrix with non-zero elements $\left(\lambda_{1}, \ldots, \lambda_{I}>0\right), \mathbf{A}_{I}$ is an $N X I$ matrix, and $\mathbf{B}_{I}$ is a [3(M-L+1)-F] $I$ matrix. As defined in Eqs.(3-69) and (3-70), $\mathbf{A}_{I}$ and $\mathbf{B}_{I}$ are constructed from normalized eigenvectors, which should be distinguished from the local eigenfunctions. Then using the generalized inverse matrix defined by

$$
\breve{\mathbf{D}}_{I}^{-1}=\mathbf{B}_{I} \Lambda_{I}^{-1} \mathbf{A}_{I}^{t}
$$

we have a solution: 


$$
\breve{\boldsymbol{x}}_{I}=\breve{\mathrm{D}}_{I}^{-1} \breve{\mathrm{y}}=\left(\mathbf{B}_{I} \Lambda_{I}^{-1} \mathbf{A}_{I}^{t}\right) \breve{y}
$$

In this process, the non-uniqueness of the solution arising from the underdetermined system is dealt with so that the norm of the resulting vector, $|y|$, becomes minimal.

The relation between the estimated value $\breve{x}_{I}$ and the exact value $x$ can be obtained by substituting Eq.(5-88) into Eq.(5-92), yielding

$$
\breve{x}_{I}=\mathbf{B}_{I} \mathbf{B}_{I}^{t} x,
$$

where Eq.(3-69) has been used. If $\mathbf{B}_{I} \mathbf{B}_{I}^{t}=\mathbf{I}$, then $\widetilde{x}_{I}=\boldsymbol{x}$ and the solution can be uniquely determined. In the underdetermined case, however, we have generally $\mathbf{B}_{I} \mathbf{B}_{I}^{t} \neq \mathbf{I}$, so that $\breve{x}_{I} \neq x$; the matrix $\mathbf{B}_{I} \mathbf{B}_{I}^{t}$ forms weighting coefficients with which $\breve{x}_{I}$ is linearly related to $\boldsymbol{x}$. Thus it is seen that the resolution of the solution $\breve{x}_{I}$ can be characterized by the socalled resolution matrix $\mathbf{B}_{I} \mathbf{B}_{I}^{t}$ [43]. As the rank of $\mathbf{B}_{I} \mathbf{B}_{I}^{t}, I$, decreases, we have lower resolution for the solution $\breve{\boldsymbol{x}}_{\boldsymbol{I}}$.

As seen from Eq.(5-92), the solution includes a component proportional to $1 / \lambda_{i}$ $(1 \leq i \leq I)$ and thus small eigenvalues induce instability in the solution. This can be easily confirmed by examining the relation between the variances of $\breve{x}$ and $\breve{y}$. Given the error $\delta \check{y}$ in the measured data, then the error observed in the solution $\delta \breve{x}_{I}$ is expressed as $\delta \breve{x}_{I}=\breve{\mathbf{D}}_{I}^{-1} \delta \breve{y}$. Thus the covariance matrices for $\breve{x}$ and $\breve{y}$ are related by

$$
\left.<\delta \breve{x}_{I} \delta \widetilde{x}_{I}^{t}\right\rangle=\breve{\mathbf{D}}_{I}^{-1}<\delta \widetilde{y} \delta \widetilde{y}^{t}>\left(\breve{\mathbf{D}}_{I}^{-1}\right)^{t}=\sigma_{y}^{2} \mathbf{B}_{I} \Lambda_{I}^{-2} \mathbf{B}_{I}^{t}
$$

where $\delta y$ is assumed to be statistically independent and has the same variance. Therefore the variance of the solution can be characterized by the matrix $\Lambda_{I}$. We see from Eq.(3-68) that if $\lambda_{i}$ is small, then the variances of $\breve{x}$ is amplified as a result of the relation given by Eq.(5-94). To obtain a stable solution, one can introduce a threshold for those eigenvalues and discard the eigenvalues smaller than this threshold as detailed in 
Refs. 48 and 77. This procedure, however, results in a decrease for the resolution of the solution due to the decrease in the number of the eigenvalues, i.e., the rank. As is well known, in general, reducing the variance leads to degraded resolution in the solution of the underdetermined inverse problem.

In connection with the stability problem, there exists a sensitivity problem among the geoacoustic parameters. Namely some parameters are extremely sensitive to variations in the input data $\Delta \kappa_{n}$ and some are not. In view of Eq.(5-88), one can immediately notice that the range variation of the parameters (output data) as well as $\Delta \kappa_{n}$ (input data) are highly dependent on their magnitudes. For example, $\Delta \mu_{j}$ is generally much smaller than $\Delta h_{j}$, because $\mu_{j}$ itself takes on a smaller value. To avoid this problem, a weighting function can be introduced into Eq.(5-88) [77]. Suppose that $\mathbf{X}$ and $\mathbf{Y}$ are the weighting matrices for $\boldsymbol{x}$ and $\boldsymbol{y}$, respectively, in Eq.(5-88), which is then transformed to

$$
\boldsymbol{y}^{\prime}=\mathbf{Y D X}^{-1} \boldsymbol{x}^{\prime}
$$

where $\boldsymbol{x}^{\prime} \equiv \mathrm{X} \boldsymbol{x}$ and $\boldsymbol{y}^{\prime} \equiv \mathbf{Y} \boldsymbol{y}$ are a new data set. Eq.(5-95) may then be solved in the same manner as Eq.(5-92). Note that this normalization operation should be made in Eq.(5-84) rather than in Eq.(5-88), because each member on the left-hand side in Eq.(587) has a different magnitude. Generally we can make use of the covariance matrix as the weighting matrix. Namely, each $\mathbf{X}$ and $\mathbf{Y}$ is defined by the square root of the corresponding covariance matrix. If the data are uncorrelated, then the weighting matrix is represented by a diagonal matrix with its standard deviation such that $\mathbf{X}_{i j}=\sigma_{x_{i}} \delta_{i j}$ and $\mathbf{Y}_{i j}=\sigma_{y_{i}} \delta_{i j}$. Actually $\sigma_{i}$ is proportional to the magnitude of the corresponding data. Thus, by utilizing the weighting matrix, we can adjust the different sensitivity of each parameter to the input data.

In this section, we developed an inverse method for determining the geoacoustic properties based on the perturbation equation for the local eigenvalues obtained in 
Section 5.4. When the total number of modes is less than the number of unknown parameters, we may make use of the SVD method to solve the underdetermined problem.

In the next section, we will apply the methods derived in this chapter to the simulated example discussed in Chapter 4.

\subsection{Analysis of simulated data}

The objective of this section is to invert for the geoacoustic parameters in our shallow water model using the local eigenvalues which were estimated by using the Hankel transform with a sliding window in Chapter 4. Before doing this, we will first examine numerically the relation between the range variation of the local eigenvalues and the geoacoustic properties by using the perturbation relation in Eq.(5-76), Section 5.4.

\subsubsection{Dependence of the local eigenvalues on the range variation of the geoacoustic parameters}

The purpose of this subsection is to understand how the range variation of the geoacoustic parameters affects the range variation of the local eigenvalues in the shallow water model used in Chapter 4.4. To do this, we examine numerically the range variation of $\kappa_{n}(x)$ by employing the perturbation relation in Eq.(5-76). Note that here we deal with this problem in the framework of a forward problem; we are therefore in the position of knowing a priori the bottom environment for this ocean model.

Since the local eigenvalue $\kappa_{n}(x)$ varies with respect to $x$ only in the present model, let us examine the range variation $\Delta \kappa_{n}(x)$ along the $x$ axis, i.e., along the radial with the azimuthal angle $\theta=0$. Figure 5-6 shows $\Delta \kappa_{n}(x)$ when setting $\Delta x=1$. As seen from the variation of $\kappa_{n}(x)$ in Figure 3-18, the $\left|\Delta \kappa_{n}(x)\right|$ of the two lowest modes is relatively small. Since most values of $\kappa_{n}(x)$ decrease with increasing range $x, \Delta \kappa_{n}(x)$ takes on a negative value except for mode 3 , whose $\Delta \kappa_{n}(x)$ has a positive value between about 2500 and $4500 \mathrm{~m}$. Hence the local eigenvalue in this region increases with $x$ as noted in Chapter 3.4.2. 
Next, by using Eq.(5-76), we can break $\Delta \kappa_{n}(x)$ into terms dependent on $\Delta h_{j}, \Delta k_{j}$, and $\Delta \mu_{j}(1 \leq j \leq 4)$ (see Section 5.4). In the present model, the water column and the lowest sediment layer are set to be range-independent and the upper two sediment layers vary with range as shown in Figures 3-10 through 3-14. Thus the terms associated with $j=1$ and $j=4$ become zero and only the terms associated with $j=2$ and $j=3$ are left in Eq.(576). In Figures 5-7 through 5-9, we show the results obtained by using Eq.(5-76) for modes 2,3 , and 5 ; mode 2 is representative of a relatively weak range variation and mode 5 corresponds to a maximum mode in the trapped modes between the sea surface and the subbottom interface (see Figure 3-15). Figures 5-7(a), 5-8(a), and 5-9(a) show the terms in Eq.(5-76) along with the $\Delta \kappa_{n}(x)$ for each mode, where the $\Delta k_{j}$ - and $\Delta \mu_{j}$-terms are added and the result is labeled by $\Delta k_{j}$. The $\Delta k_{j}$-term represents the component of $\Delta \kappa_{n}(x)$ due to the variation of the medium in the $j$ th layer, whereas the $\Delta h_{j}$-term represents a component due to the variation of the sediment interface. Note that $\Delta h_{2}(x)$ corresponds to the bathymetric variation. In Figures 5-7(b), 5-8(b), and 5-9(b), we break the $\Delta k_{j}$-term into $\Delta c_{j}$ - and $\Delta g_{j}$-terms, instead of $\Delta k_{j}$ - and $\Delta \mu_{j}$-terms, by using the relation given by Eq.(5-58). We will show below which parameters have a strong influence on $\Delta \kappa_{n}(x)$ for each mode.

\section{Case of mode 2}

In the case of mode 2 in Figure 5-7(a), we find from the curves $\Delta h_{2}$ and $\Delta k_{2}$ that the variation in the first sediment layer $(j=2)$ contributes primarily to the variation of the local eigenvalue $\Delta \kappa_{2}(x)$. This result can be inferred from the fact that the amplitude of the eigenfunction $u_{2}(x, z)$ decreases exponentially with depth below the interface $h_{3}(x)$, as shown in Figure 3-15; therefore, mode 2 is less sensitive to the second sediment layer.

Figure 3-15 also indicates that $\partial u_{2} /\left.\partial z\right|_{m_{2}}$, which stands for the vertical particle velocity at the water-bottom interface, comes close to zero. The $\partial u_{2} /\left.\partial z\right|_{m_{2}}$-term in Eq.(5- 
76) also comes close to zero, so that the coefficient of $\Delta h_{2}(x)$ takes on a negative value in the present case because of these two reasons:

1. $\rho_{1}<\rho_{2}$, i.e., the water density is smaller than the density in the first sediment layer.

2. $\left.\gamma_{2}^{2}\right|_{m_{2}-0}>\left.\gamma_{2}^{2}\right|_{m_{2}+0}$ due to $c_{1}<c_{2}$, i.e., the sound speed in the water is smaller than that in the first sediment layer.

Recalling the condition that the water depth increases monotonically with $x$, we see that $\Delta h_{2}(x)>0$ and so the $\Delta h_{2}$-term takes on a negative value. As a result, the $\Delta h_{2}$-term contributes to $\Delta \kappa_{2}(x)$ so that the local eigenvalue $\kappa_{2}(x)$ decreases with range $x$.

The $\Delta k_{2}$-term changes its sign at about $x=2900 \mathrm{~m}$ and $4300 \mathrm{~m}$. This occurs for two reasons: (1) the $\Delta k_{2}$-term is dominated by the $\Delta c_{2}$-term as shown in Figure 5-7(b), and (2) $c_{2}(x)$ changes from a decreasing value to an increasing one at about $x=2900 \mathrm{~m}$ and again changes at about $x=4300 \mathrm{~m}$, as seen from Figure 3-11. Thus, in this range, the $\Delta k_{2}$ term contributes to $\Delta \kappa_{2}(x)$ so that the local eigenvalue $\kappa_{2}(x)$ increases with range $x$.

In particular, at about $3500 \mathrm{~m}$, the $\Delta h_{2}$ - and $\Delta k_{2}$-terms have the same order of magnitude but have opposite signs, so that $\Delta \kappa_{2}(x)$ comes close to zero. As a result, $\kappa_{2}(x)$ remains constant around this range.

\section{Case of mode 3}

As for mode 3, we can see from Figure 5-8(a) that its local eigenvalue is more strongly affected by the second sediment layer $(j=3)$ as compared to the local eigenvalue of mode 2; the $\Delta k_{3}$-term has an amplitude comparable to the $\Delta k_{2}$-term for ranges less than $x=2000 \mathrm{~m}$. Also, we observe that the amplitude of the $\Delta h_{3}$-term is larger than that of the $\Delta h_{2}$-term. This mode, therefore, is more sensitive to the variation of the sediment interface than to the bathymetric variation.

Figure 5-8(b) shows that the $\Delta k_{2}$-term depends on the variation of both $c_{2}(x)$ and $g_{2}(x)$, whereas the $\Delta k_{3}$-term depends on the variation of only $c_{3}(x)$. Namely, mode 3 is less sensitive to the variation of the sound speed gradient $g_{3}(x)$. On the other hand, the 
$\Delta g_{2}$-term changes its sign at about $x=2400 \mathrm{~m}$ because $g_{2}(x)$ changes from an increasing value to a decreasing one at that range, as seen from Figure 3-12.

\section{Case of mode 5}

As for mode 5 , the effect of the $\Delta k_{3}$-term is amplified, which can be expected from the mode shape $u_{5}(x, z)$ in Figure 3-15. It is found from Figure 5-9(a) that the $\Delta k_{3}$-term dominates $\Delta \kappa_{5}(x)$ for ranges less than $2200 \mathrm{~m}$. As seen from Figure 5-9(b), the $\Delta k_{3}$ term is dominated by the $\Delta c_{3}$-term. We can see, therefore, that mode 5 is most sensitive to the variation of $c_{3}(x)$ in this range.

In this subsection, we demonstrated with an example how the range variation of the sediment properties affect the range variation of the local eigenvalues in our shallow water model. In the next subsection, by assuming that the geoacoustic parameters in this model are unknown, we will use the inverse method developed in Section 5.5 to determine the parameters from the local eigenvalues.

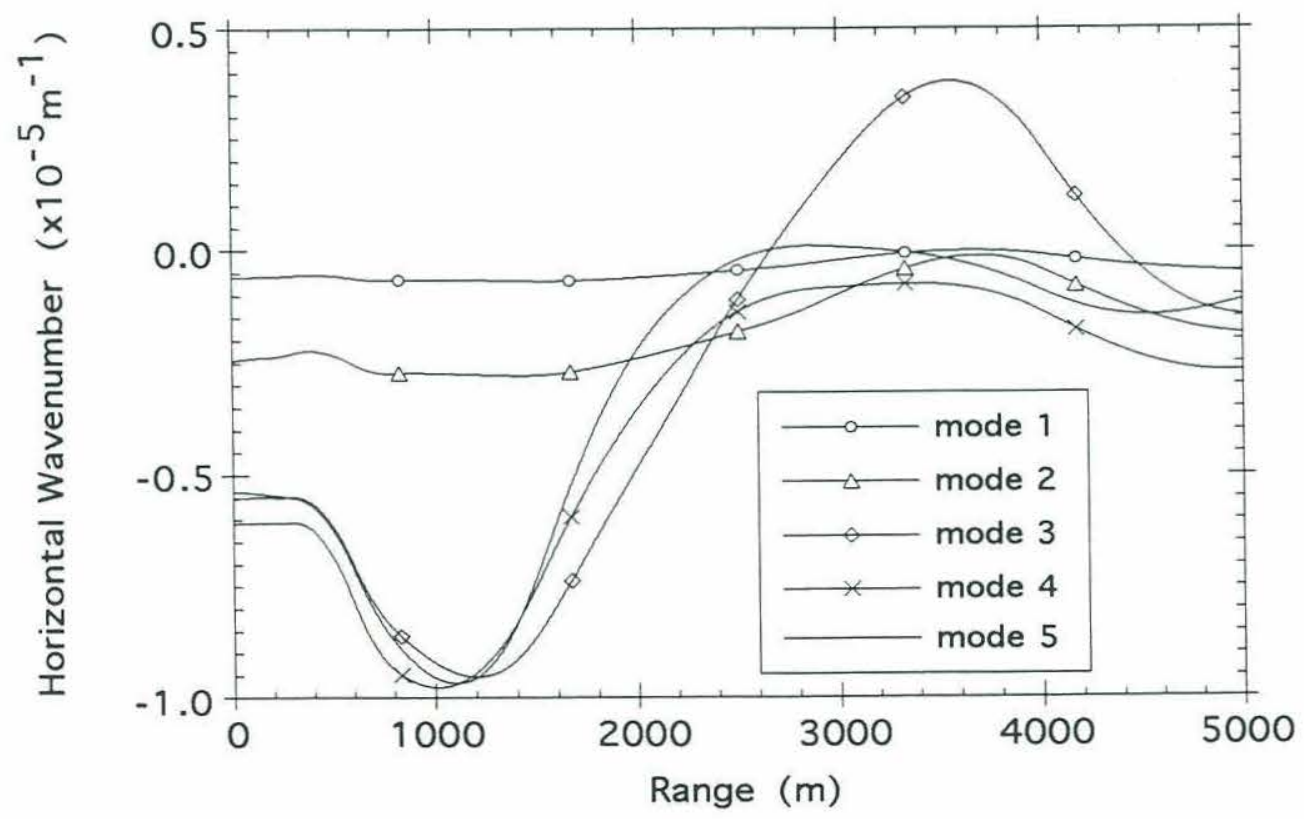

Figure 5-6: Perturbed local eigenvalue $\Delta \kappa_{n}(x)$ in the shallow water model. 


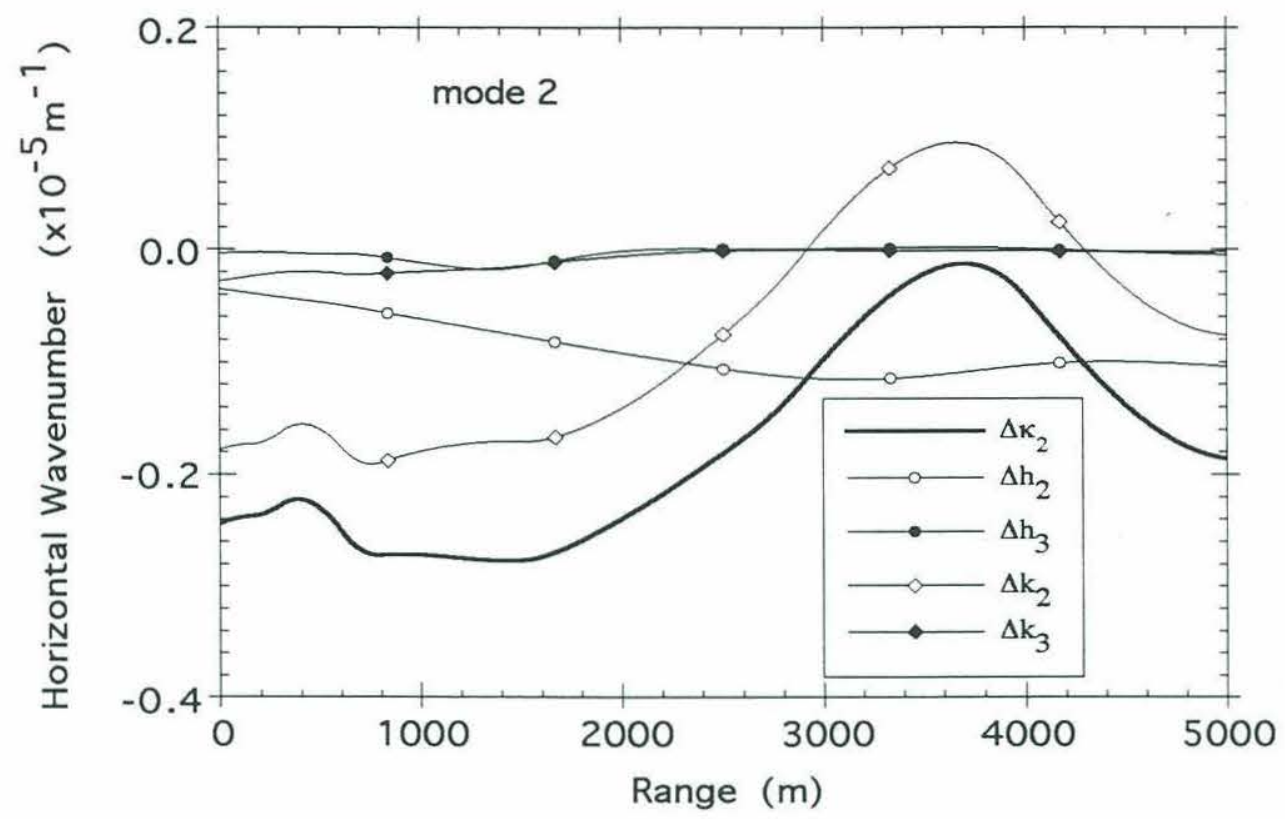

(a)

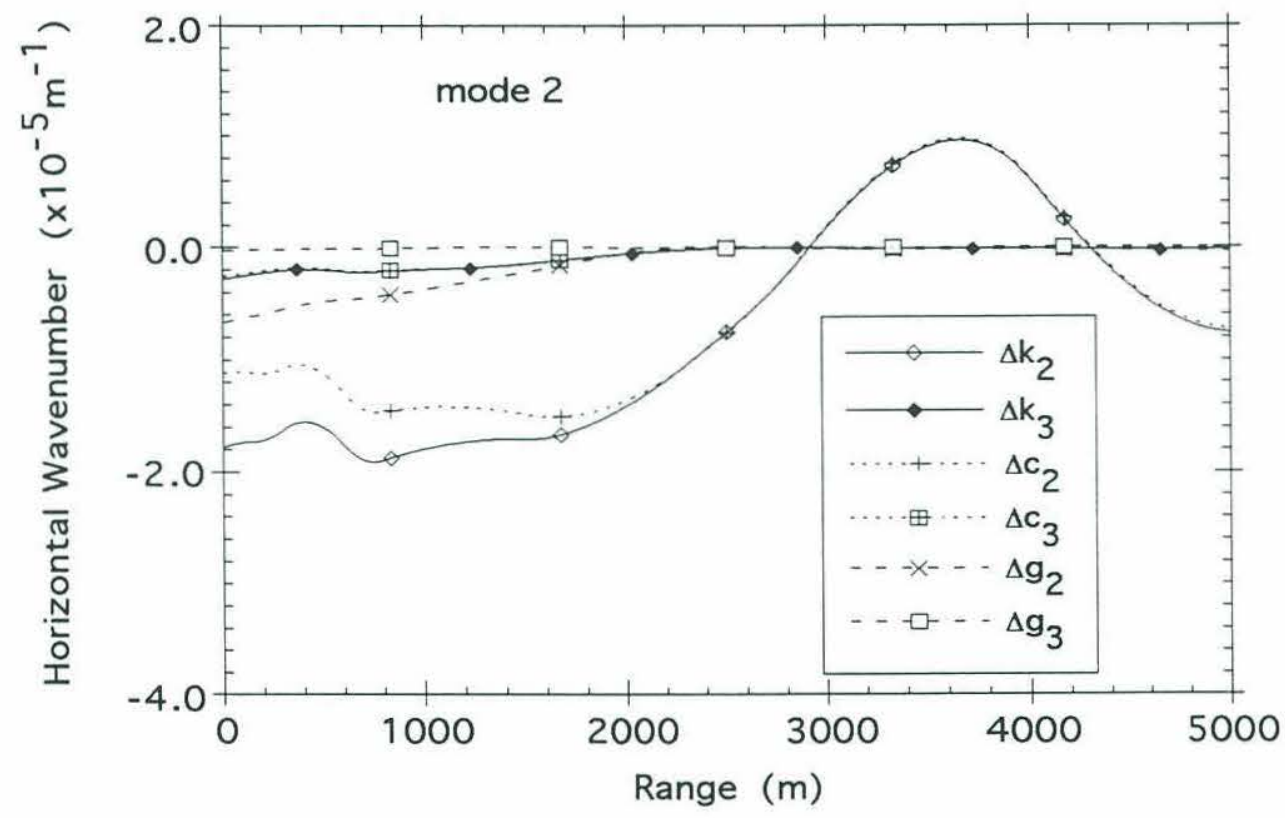

(b)

Figure 5-7: (a) Decomposition of the perturbed local eigenvalue $\Delta \kappa_{2}(x)$ in Figure 5-6 into the $\Delta h_{j}$ - and $\Delta k_{j}$-terms. (b) Decomposition of the $\Delta k_{j}$-term into the $\Delta c_{j}$ - and $\Delta g_{j}$-term. 


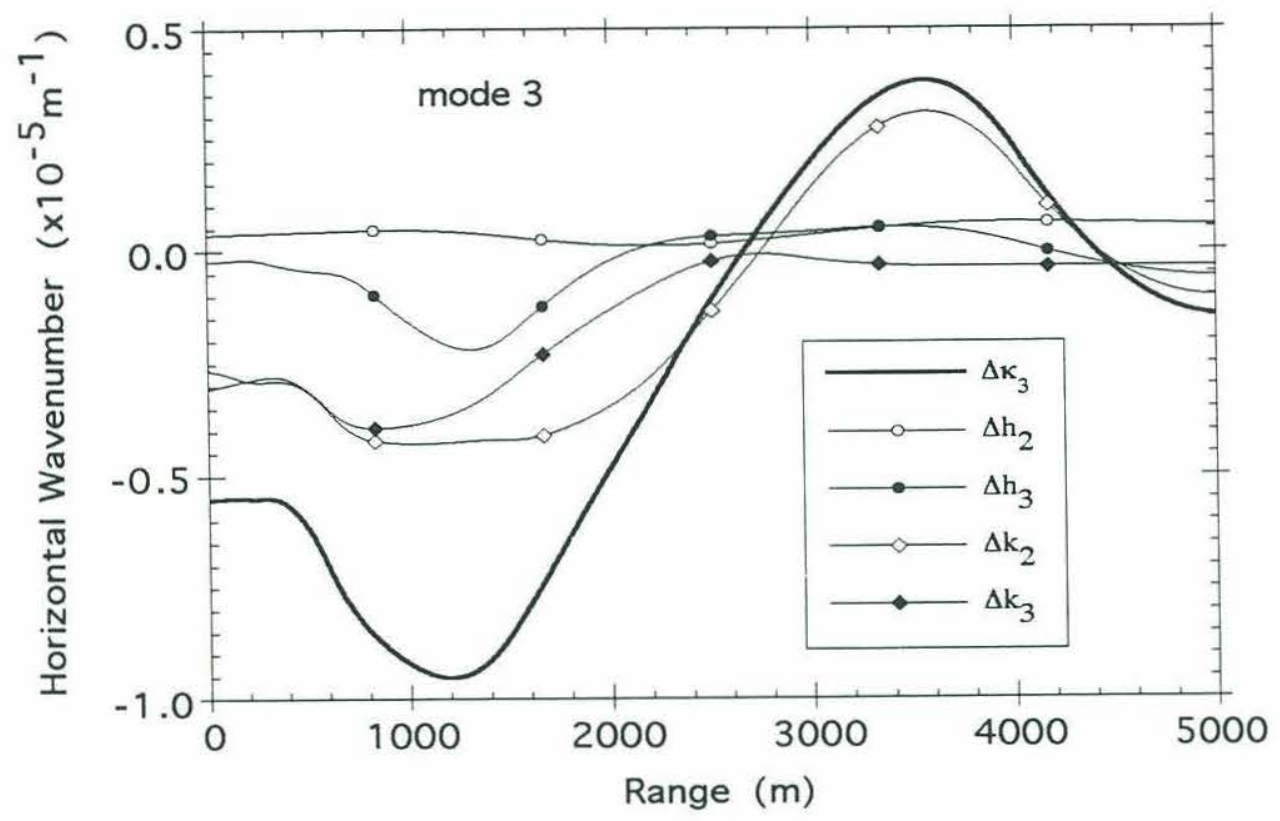

(a)

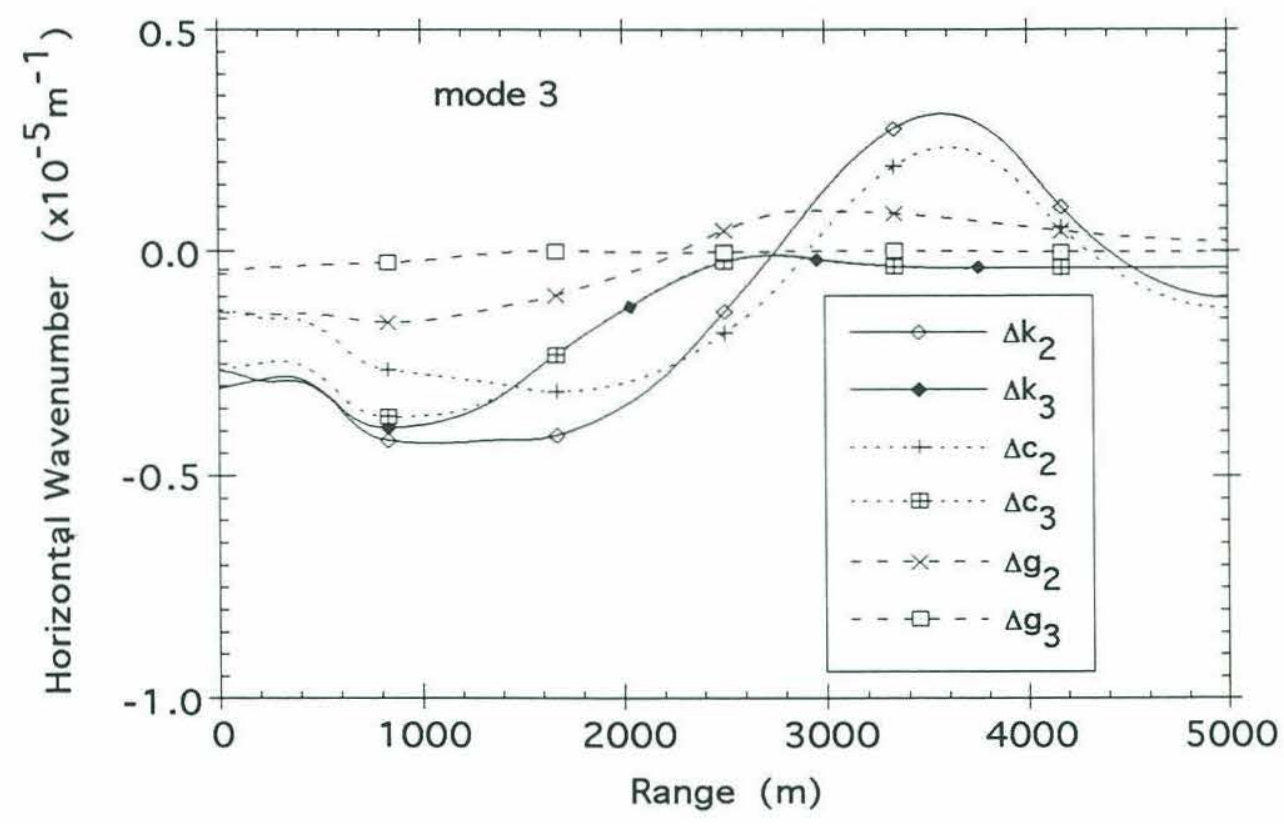

(b)

Figure 5-8: (a) Decomposition of the perturbed local eigenvalue $\Delta \kappa_{3}(x)$ in Figure 5-6 into the $\Delta h_{j}$ - and $\Delta k_{j}$-terms. (b) Decomposition of the $\Delta k_{j}$-term into the $\Delta c_{j}$ - and $\Delta g_{j}$-term. 


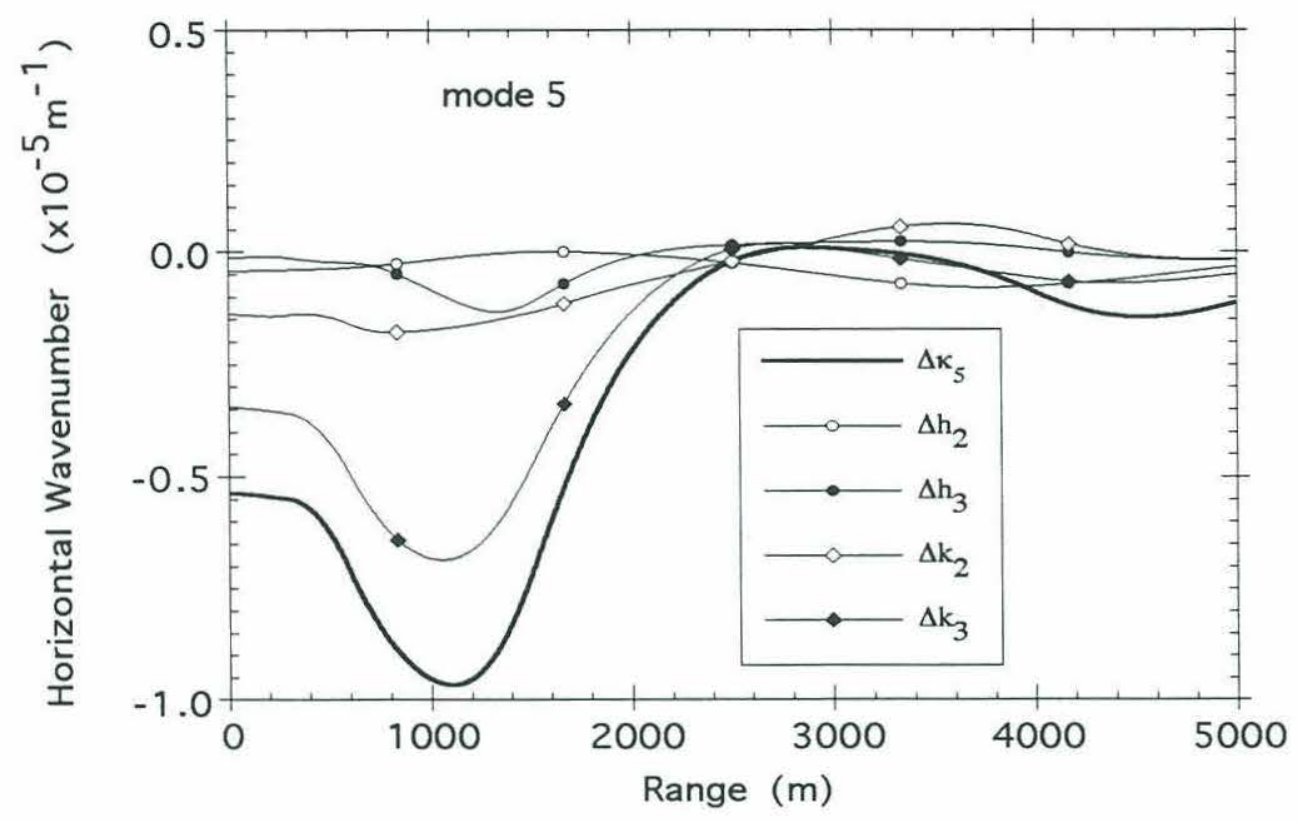

(a)

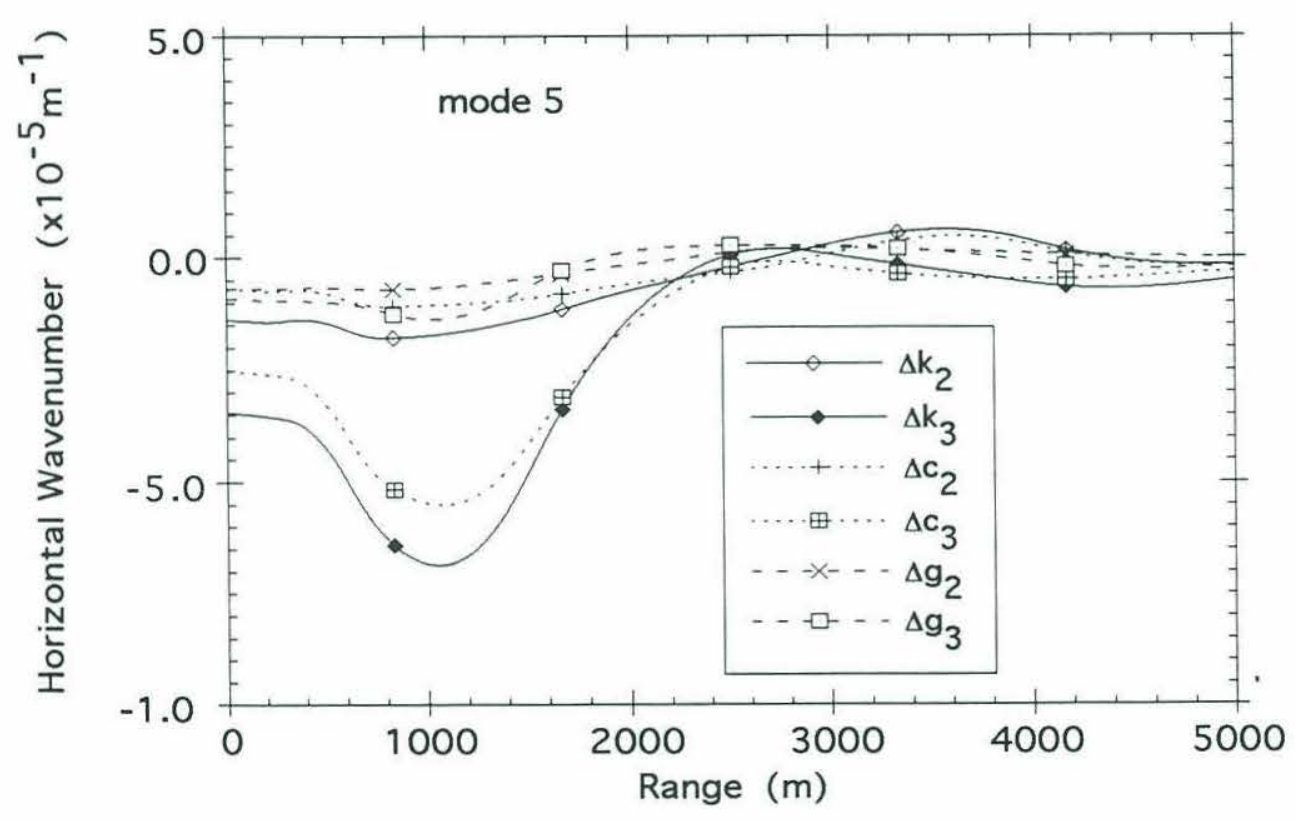

(b)

Figure 5-9: (a) Decomposition of the perturbed local eigenvalue $\Delta \kappa_{5}(x)$ in Figure 5-6 into the $\Delta h_{j}$ - and $\Delta k_{j}$-terms. (b) Decomposition of the $\Delta k_{j}$-term into the $\Delta c_{j}$ - and $\Delta g_{j}$-term. 


\subsubsection{Inversion for the geoacoustic parameters}

In this subsection, let us assume that the geoacoustic parameters are unknown in the shallow water model used in Chapter 4 except for the region close to the array of receivers. The purpose of this section is to estimate numerically those parameters by using the inverse method developed in Section 5.5.

For input data, we can use the local eigenvalues, which were estimated in Chapter 4.4 by applying the Hankel transform to the pressure field in a wedge-type bottom environment. In that chapter we also focused on the azimuthal direction of $\theta=70^{\circ}$ and tried to detect the local eigenvalues along a radial in this direction. First we estimated the local eigenvalues by using the zero-order Hankel transform with a sliding window (Figure 4-10); then we compensated for the deficit in the estimated values by using the general Hankel transform with a 2-D sliding window (Figure 4-18). In the current section, we estimate the geoacoustic parameters by using these uncompensated and compensated values as input data, then we compare these two sets of estimated parameters.

In order to utilize the inverse method given by Eq.(5-83) or Eq.(5-87), we need to provide the range variation of the local eigenvalue $\Delta \kappa_{n}(r)$ as input data. When taking $\Delta \kappa_{n}(r)$ in accordance with Eq.(5-82), we have to choose the distance $\Delta r$ so that $\Delta \kappa_{n}(r)$ is within the range of linear variation. This is due to the fact that the inverse method developed in Section 5.4 is based on linear perturbation theory. But too small a step for $\Delta r$ leads to an inefficient calculation. Therefore let us first approximate $\kappa_{n}(r)$ by a linear curve fitting every mode. Figure 5-10 shows these results for the estimated local eigenvalues. The dots $(\cdot)$ represent the compensated peak positions in wavenumber, which were obtained by using the general Hankel transform with a sliding window in Chapter 4.4; the segmented straight lines stand for the linear fits to those data, where the portion between the points $(\diamond)$ has a constant gradient $\left(\partial \kappa_{n} / \partial r\right)$. These points are determined in accordance with the variation of $\kappa_{n}(r)$; i.e., when it changes nonlinearly, 
we need to represent it by shorter line segments. We can then sample $\kappa_{n}\left(r_{\ell}\right)$ at $r_{\ell}$ on each linear segment at a suitable interval $\Delta r_{\ell}$. Here we set $\Delta r_{\ell}$ so that $\Delta r_{\ell}$ does not exceed $100 \mathrm{~m}$. Note that $\Delta r_{\ell}$ is not necessarily constant and can vary depending on the variation of $\kappa_{n}(r)$.

The input data $\Delta \kappa_{n}\left(r_{\ell}\right)$ determined in this way are then used in Eq.(5-87). Since the water column $(j=1)$ and the subbottom $(j=4)$ are assumed to be range-independent in the present model, we can set $\Delta h_{4}, \Delta c_{4}, \Delta g_{4}$, and $\Delta v_{n}$ defined in Eq.(5-81) to be zero.

Besides, we can assume that the bathymetric variation is known a priori and so $\Delta \widehat{h}_{2}$ is placed on the left-hand side of Eq.(5-87). Hence we have a total of five unknowns: $\Delta \breve{c}_{2}$ and $\Delta \breve{g}_{2}$ in the first sediment layer, and $\Delta \breve{c}_{3}, \Delta \breve{g}_{3}$, and $\Delta \breve{h}_{3}$ in the second sediment layer. Since the total number of given modes is five, $\breve{\mathbf{D}}$ in Eq.(5-88) becomes a $5 \times 5$ regular matrix. Here let us apply the normalization procedure in Eq.(5-95) to $\breve{\mathbf{D}}$ in order to reduce the difference in the magnitudes of those variations and to obtain a reliable numerical solution. Then, by solving Eq.(5-88) at each $r_{\ell}$, we can determine those unknown parameters at each range. Figures 5-11(a)-(d) compare these inverse solutions with exact ones, which were provided in Chapters 3.4 and 4.4. In Figures 5-11(c)-(d), note that $\tilde{c}_{2}$ and $\tilde{c}_{3}$ represent the sound speeds at the sediment interface depth $h_{2}(r)$ and $h_{3}(r)$, respectively, instead of $c_{2}$ and $c_{3}$ at fixed depths defined in Section $5.4 ; \tilde{c}_{j}(j=2,3)$ can be calculated by using Eq.(5-51) since we have estimated $c_{j}$ along with the gradient $g_{j}$ in each layer. It can be observed that the estimated parameters take on values close to the exact values. The difference between the two curves is due to the approximation of the input data $\Delta \kappa_{n}\left(r_{\ell}\right)$. The 3-D pictures of the sound speed profile in the sediment are also compared in Figures 5-12(a) and (b) and are almost identical.

\section{Case of uncompensated local eigenvalues}

Here let us examine the case where we use the uncompensated, estimated values for the local eigenvalues as input data for the inverse method; these were obtained by using 
Table 5-1: Matrix component in Eq.(5-94)

$\begin{array}{cc}\text { parameter } & \text { (diagonal term) } \\ & \\ h_{3} & 6.83 \\ g_{2} & 13.66 \\ g_{3} & 3.86 \\ c_{2} & 5.83 \\ c_{3} & 4.62\end{array}$

the zero-order Hankel transform in Chapter 4.4, as shown in Figure 4-10. By following the same procedure as above, we can determine the unknown geoacoustic parameters. The results are shown in Figure 5-13. As compared to the above compensated case, we observe that the difference between the estimated and exact parameters has increased noticeably. This difference increases with range as the error in the estimate of $\kappa_{n}(r)$ increases with range. In particular, the difference in the gradient of the first sediment layer, $g_{2}$, is prominent. This can be understood from Table 5-1, where the square root of the diagonal term in the matrix in Eq.(5-94) is listed. The variance of the estimated parameters is proportional to this component; the value for $g_{2}$ is the largest, which accounts for the prominent difference.

\section{Underdetermined case}

Next we will study the case of an underdetermined problem, in which the total number of modes is less than the number of unknown parameters. Suppose that the compensated value of the local eigenvalues is given, but only four modes are available in total. As shown in Eq.(5-92), we can make use of the SVD method to solve this 
underdetermined problem. Here let us consider the following two cases: one is the case where modes 1-4 are employed and the other is the case where modes 2-5 are employed. Figures 5-14 and 5-15 show the results of applying the SVD method to each case. Figure 5-16 also compares the 3-D pictures of the sound speed profile in the sediment bottom in each case. In the former case, we have a relatively large error, especially for ranges greater than $2000 \mathrm{~m}$. On the contrary, in the latter case, we obtain almost the same result as for the determined case in Figure 5-10. As seen from a comparison of these two results, we can deduce that mode 5 carries more information about the sediment properties of the second layer than mode 1 . This is because the turning point depth of mode 1 is located inside the first sediment layer and so this mode is less sensitive to the range variation of the second sediment layer.

In this subsection, we demonstrated through simulated examples that we can accurately estimate the unknown parameters in our shallow water model by using the inverse method developed in Section 5.5. Next we observed that the error in the estimated parameters increases when using the underestimated local eigenvalues as input data, which were obtained by using the zero-order Hankel transform with a sliding window in Chapter 4.4. Finally, we showed that the SVD method works well for the underdetermined case as long as we choose the modes properly. 


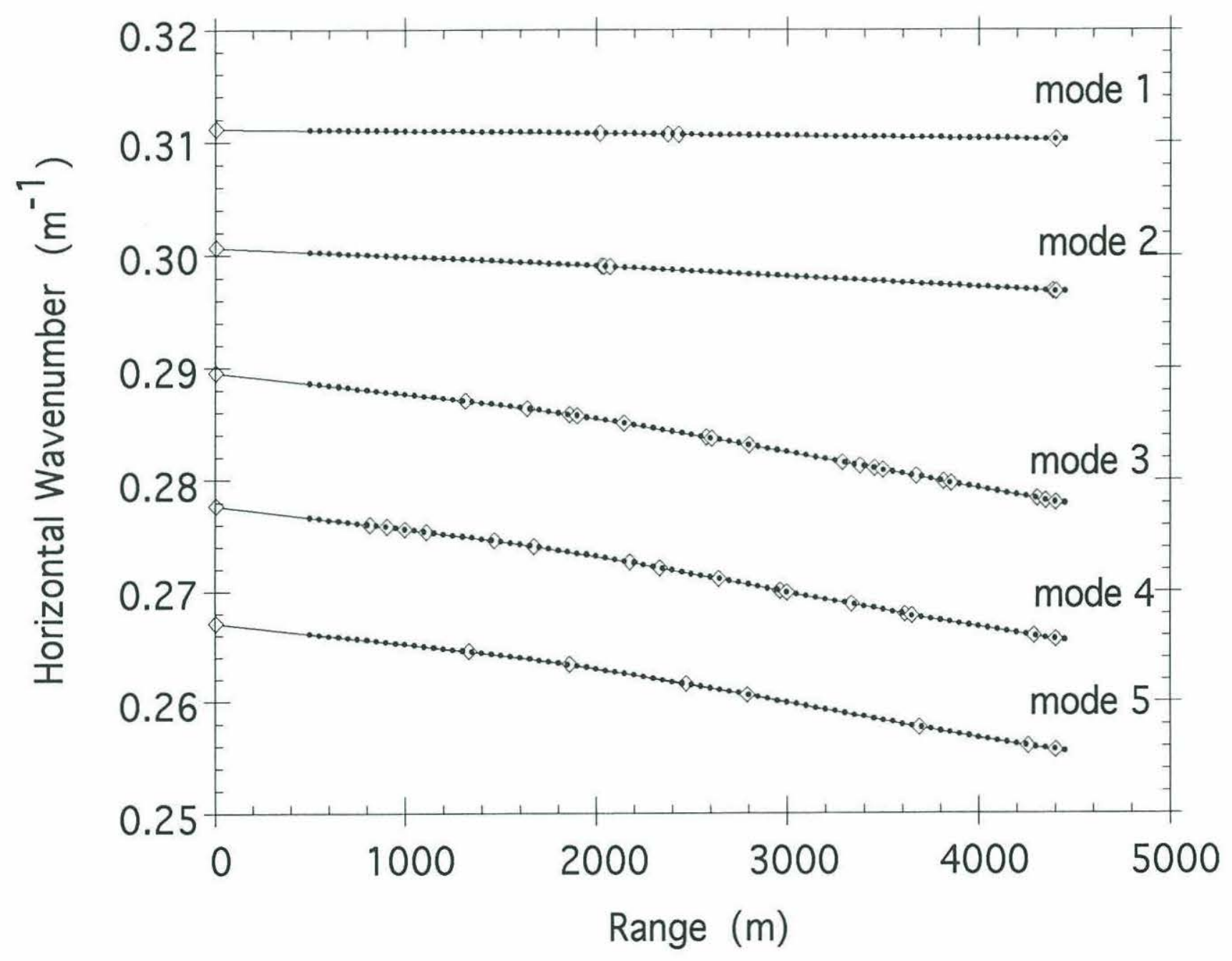

Figure 5-10: Estimated local eigenvalues obtained by using the general Hankel transform with a sliding window (• ) and linear fits to these eigenvalues $(-\diamond)$. 


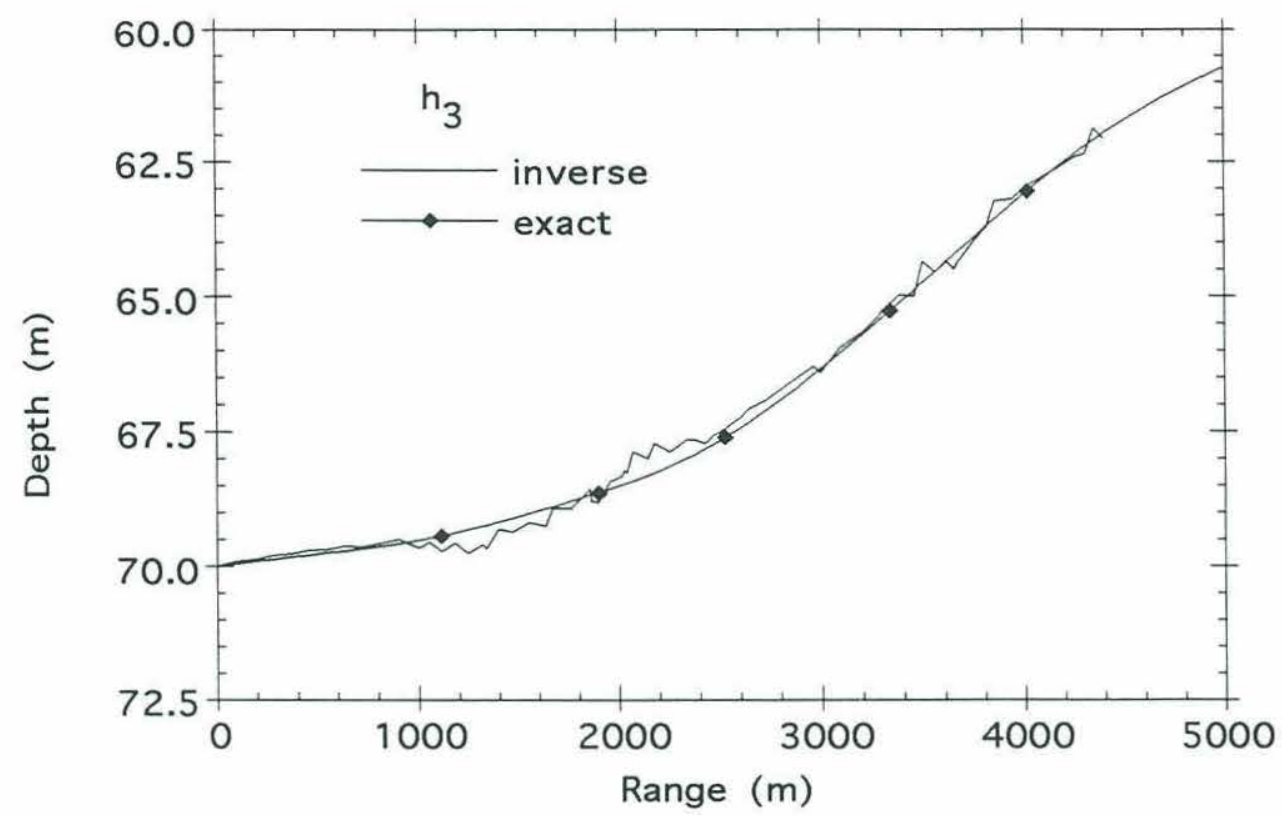

(a)

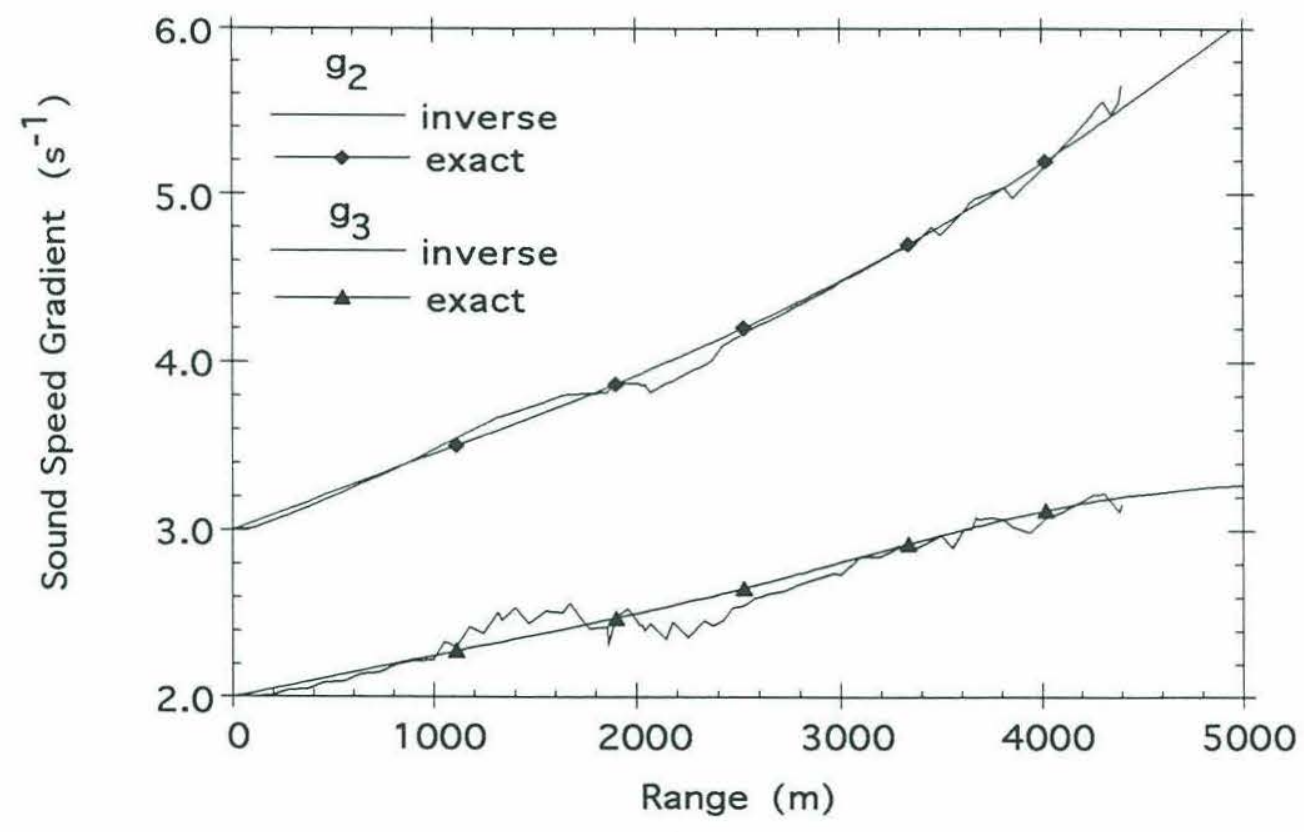

(b)

Figure 5-11: Comparison of the exact and estimated geoacoustic parameters in the shallow water model. The compensated local eigenvalues are used for the estimation of parameters. (a) Sediment interface depth $h_{3}$, (b) sound speed gradients $g_{2}$ and $g_{3}$, (c) sound speed $\tilde{c}_{2}$ at interface depth $h_{2}$, and (d) sound speed $\tilde{c}_{3}$ at interface depth $h_{3}$. 


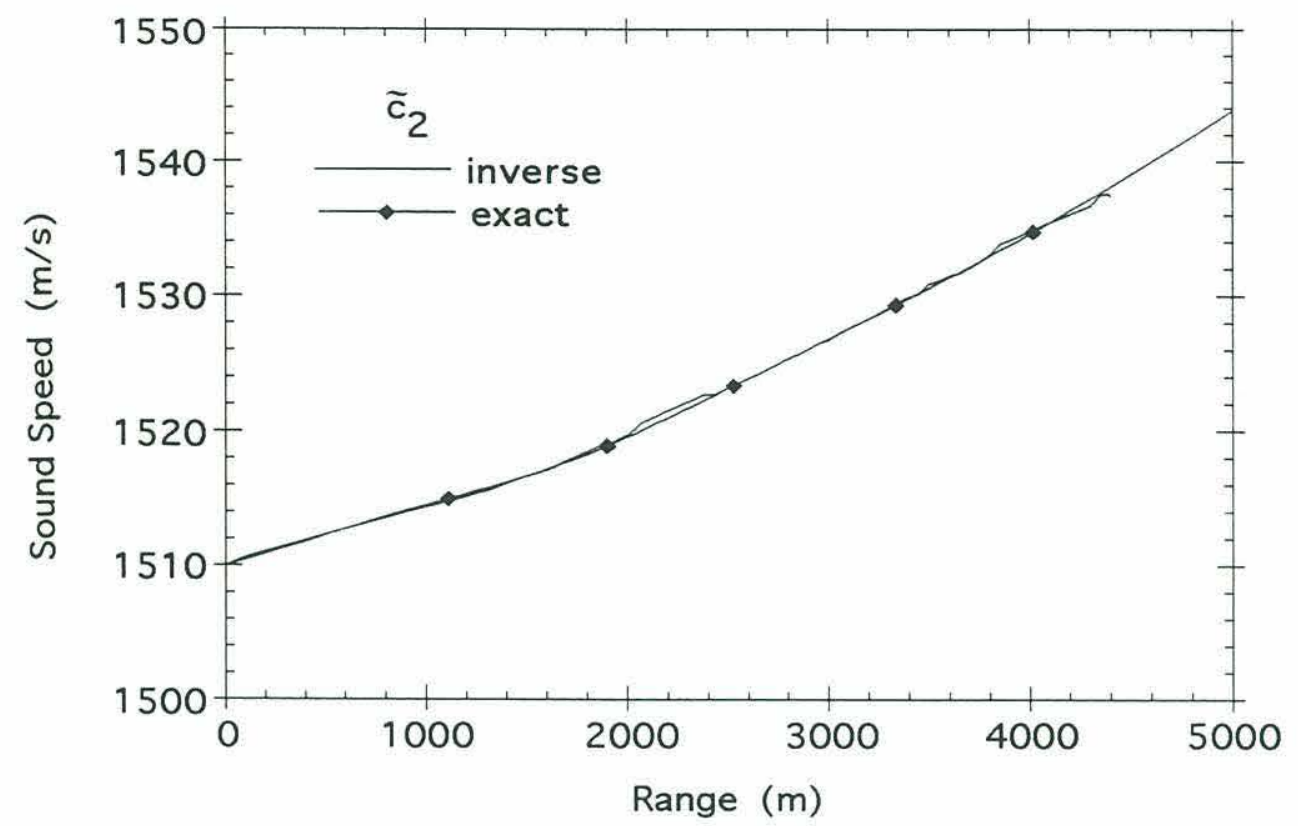

(c)

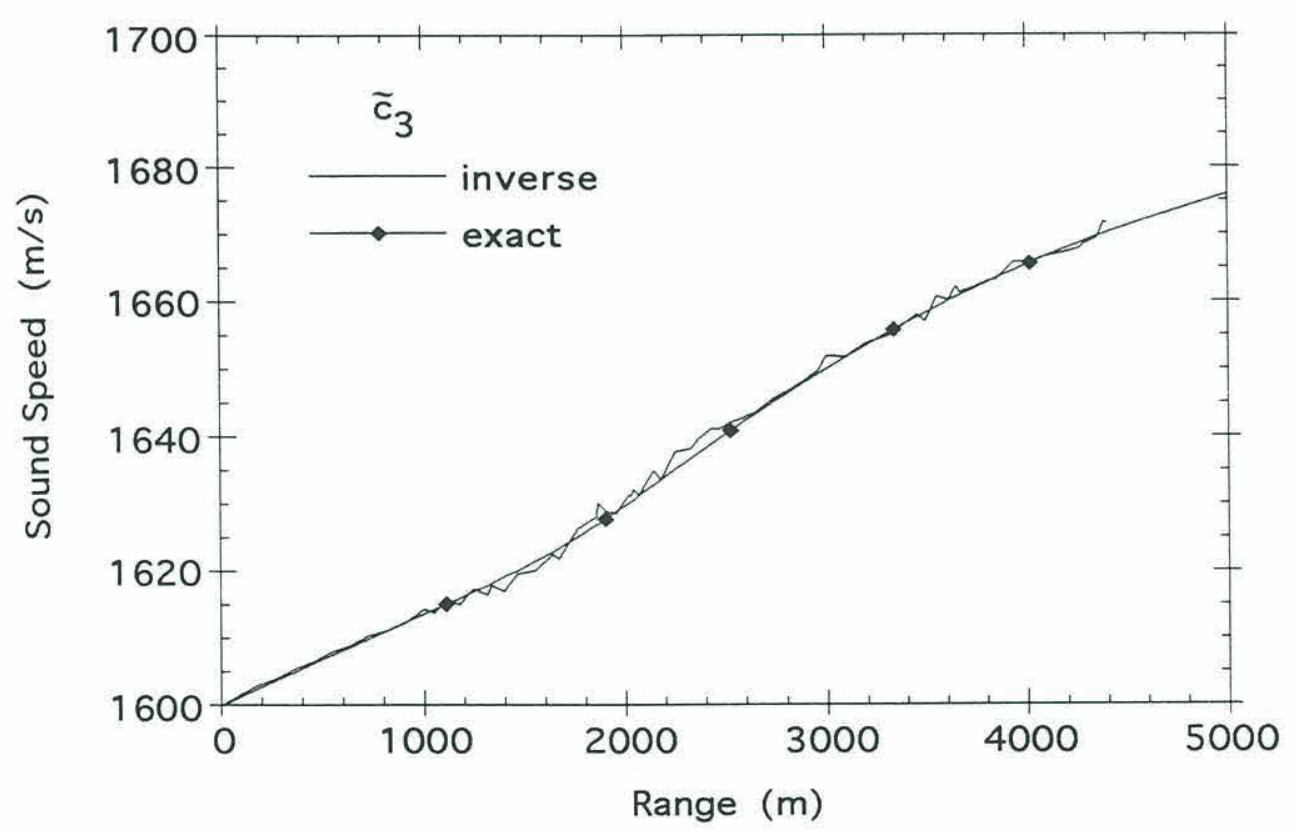

(d)

Figure 5-11, continued. 

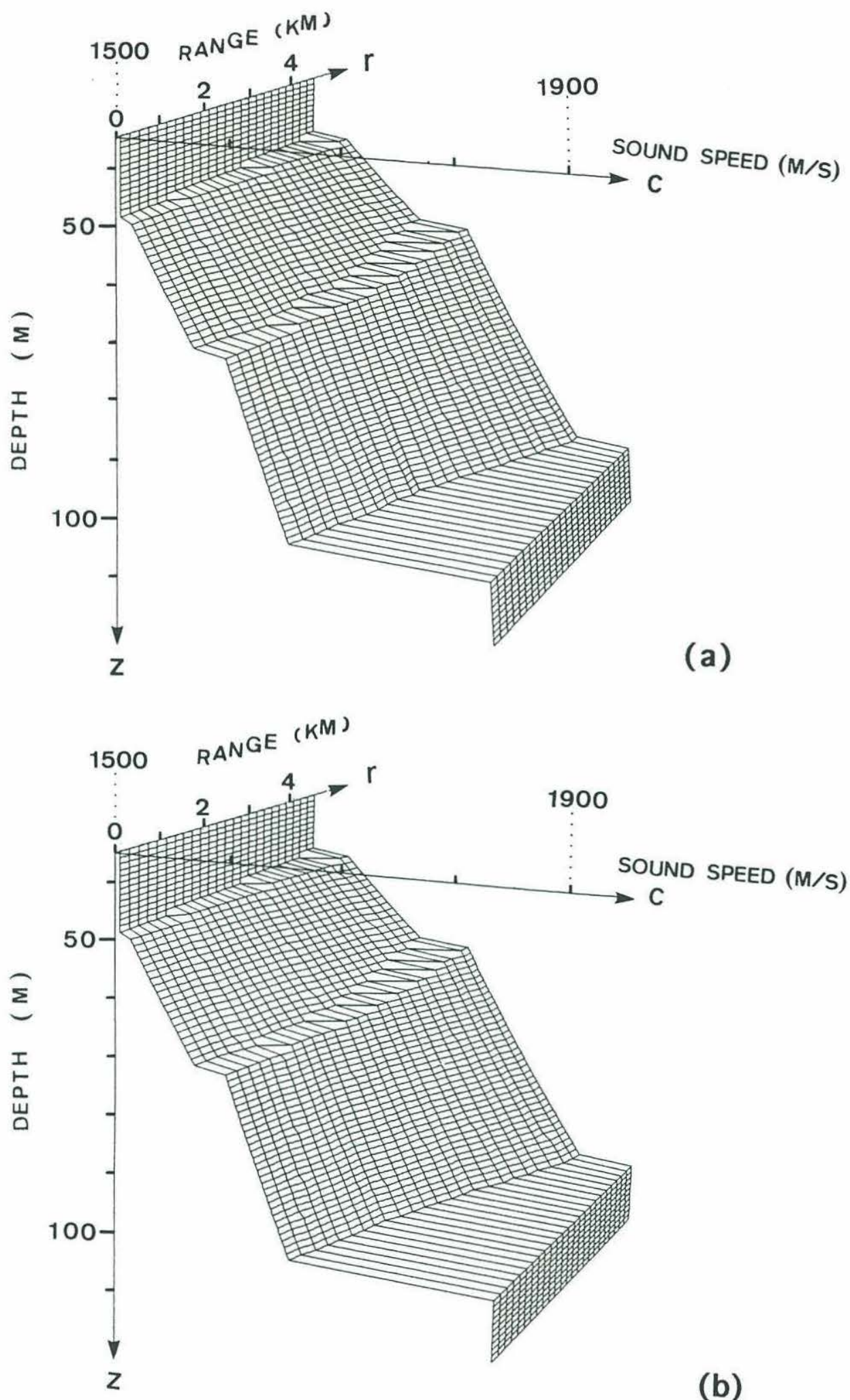

Figure 5-12: Comparison of 3-D pictures of the sound speed profiles in the sediment bottom. (a) Estimated and (b) exact profiles. 


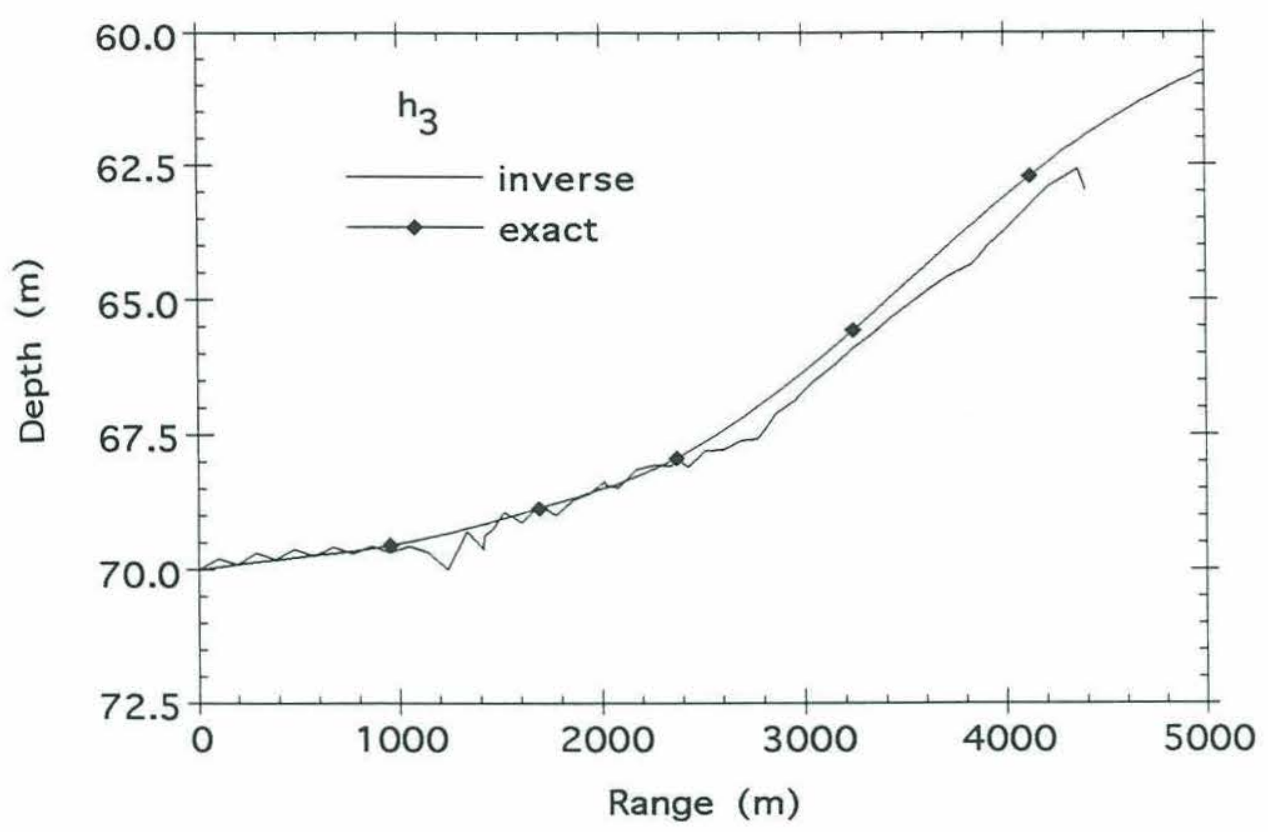

(a)

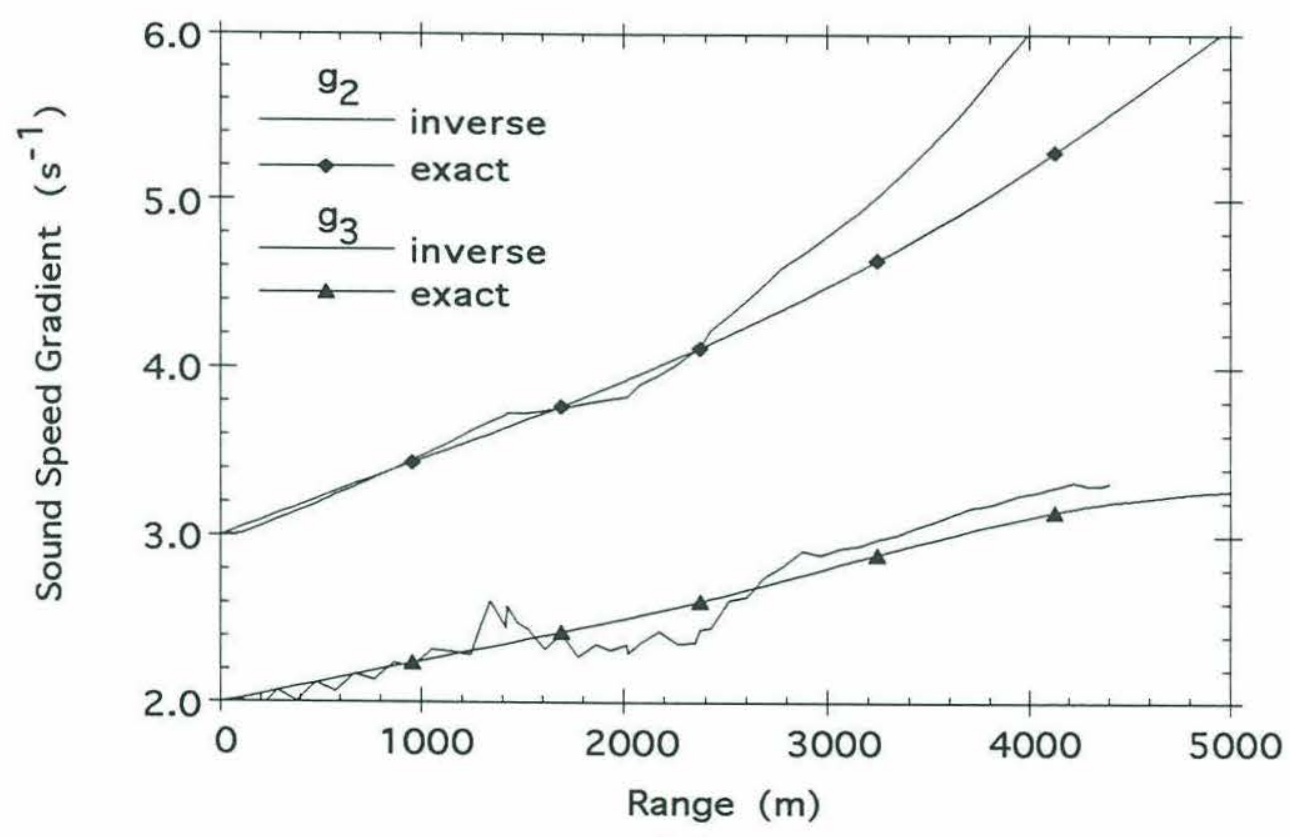

(b)

Figure 5-13: Comparison of the exact and estimated geoacoustic parameters in the shallow water model. The uncompensated local eigenvalues are used for the estimation of parameters. (a) Sediment interface depth $h_{3}$, (b) sound speed gradients $g_{2}$ and $g_{3}$, (c) sound speed $\tilde{c}_{2}$ at interface depth $h_{2}$, and (d) sound speed $\tilde{c}_{3}$ at interface depth $h_{3}$. 


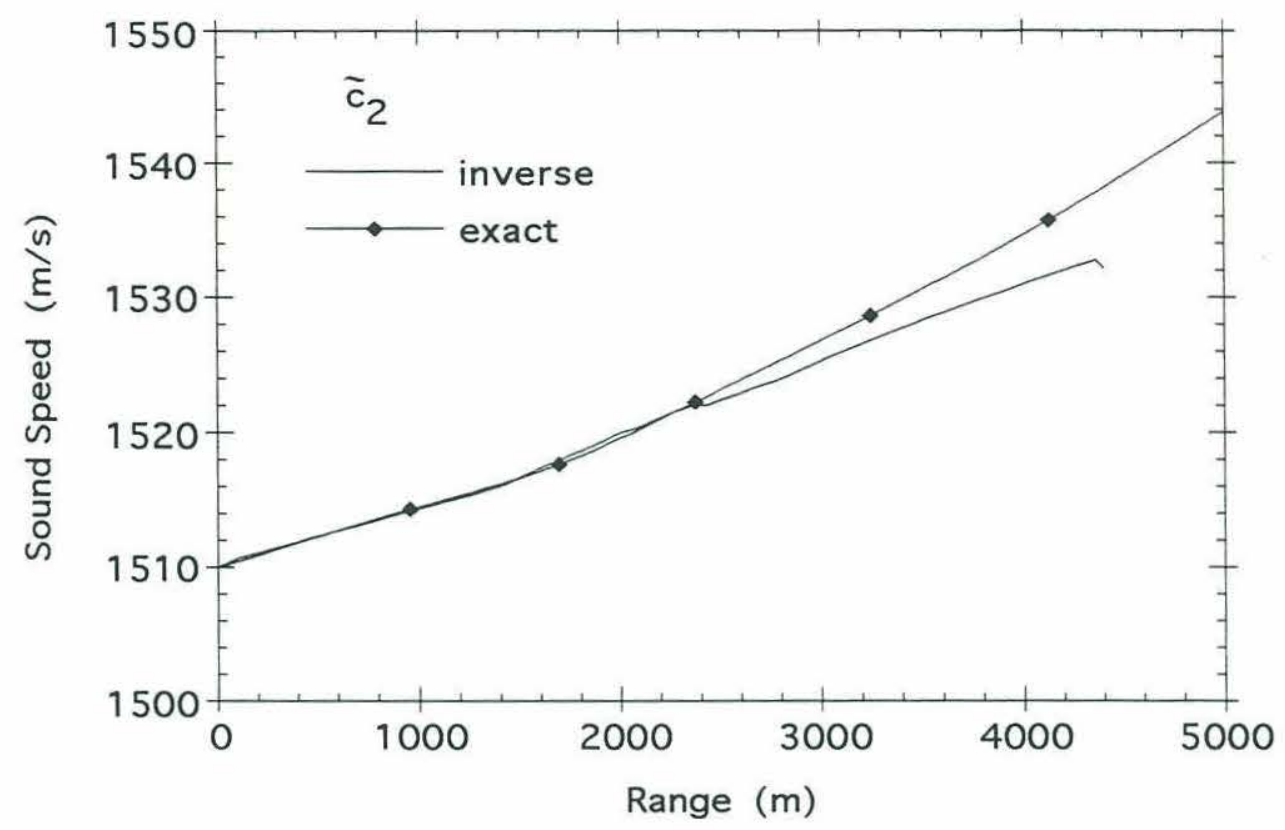

(c)

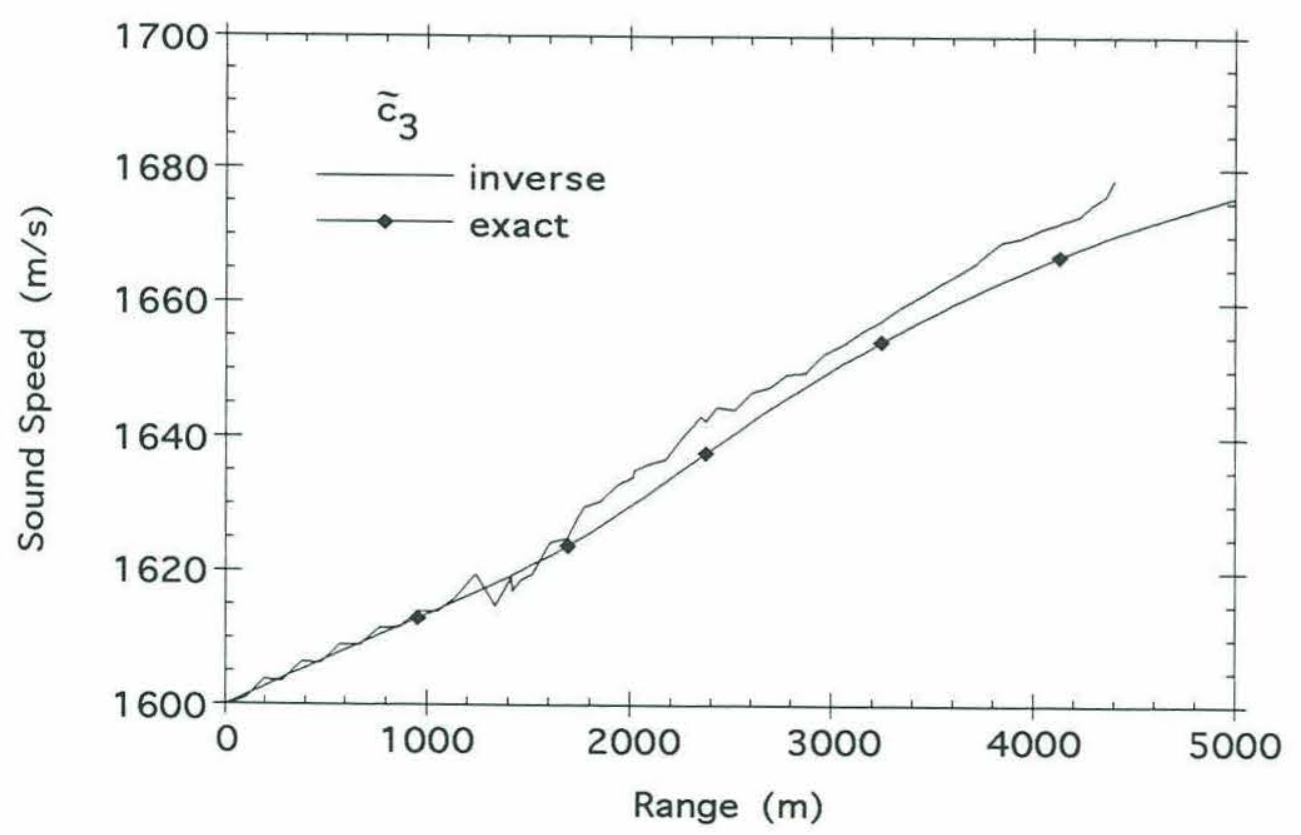

(d)

Figure 5-13, continued. 


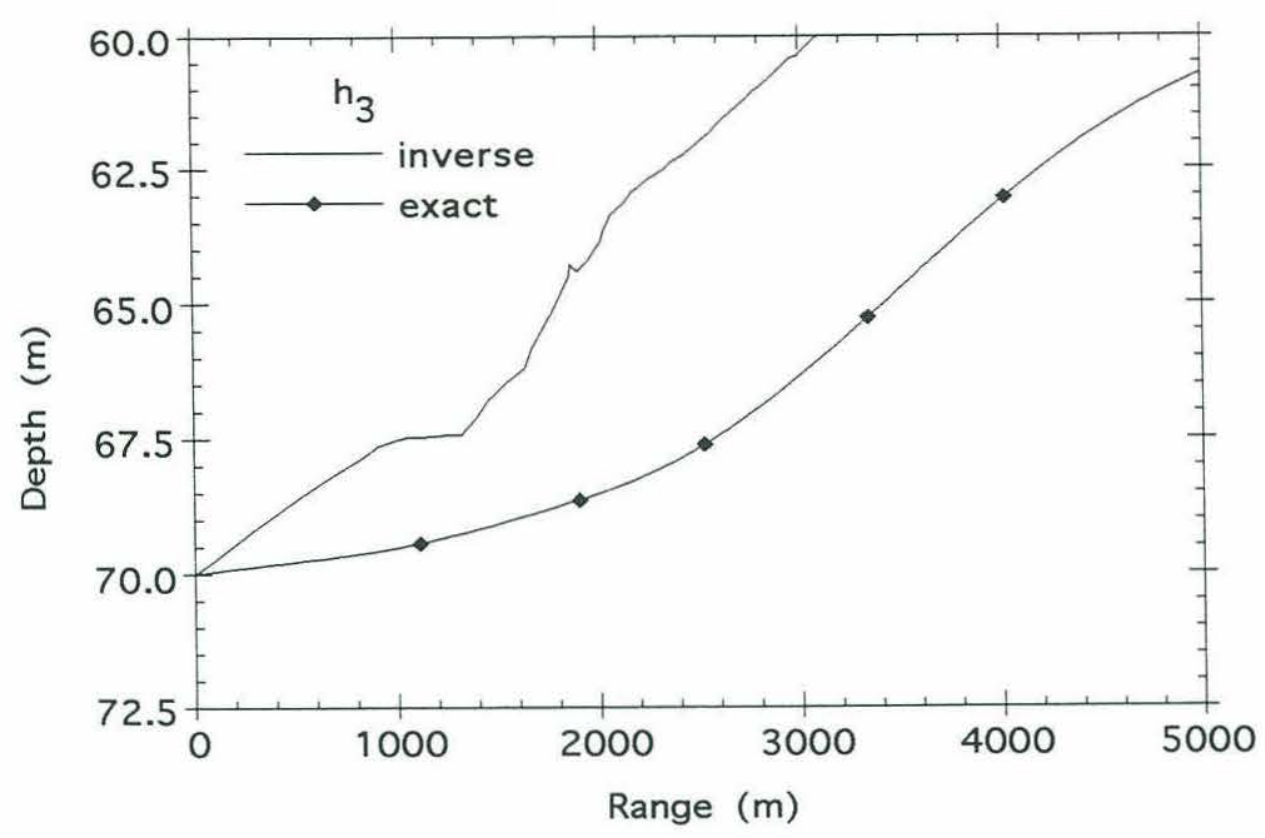

(a)

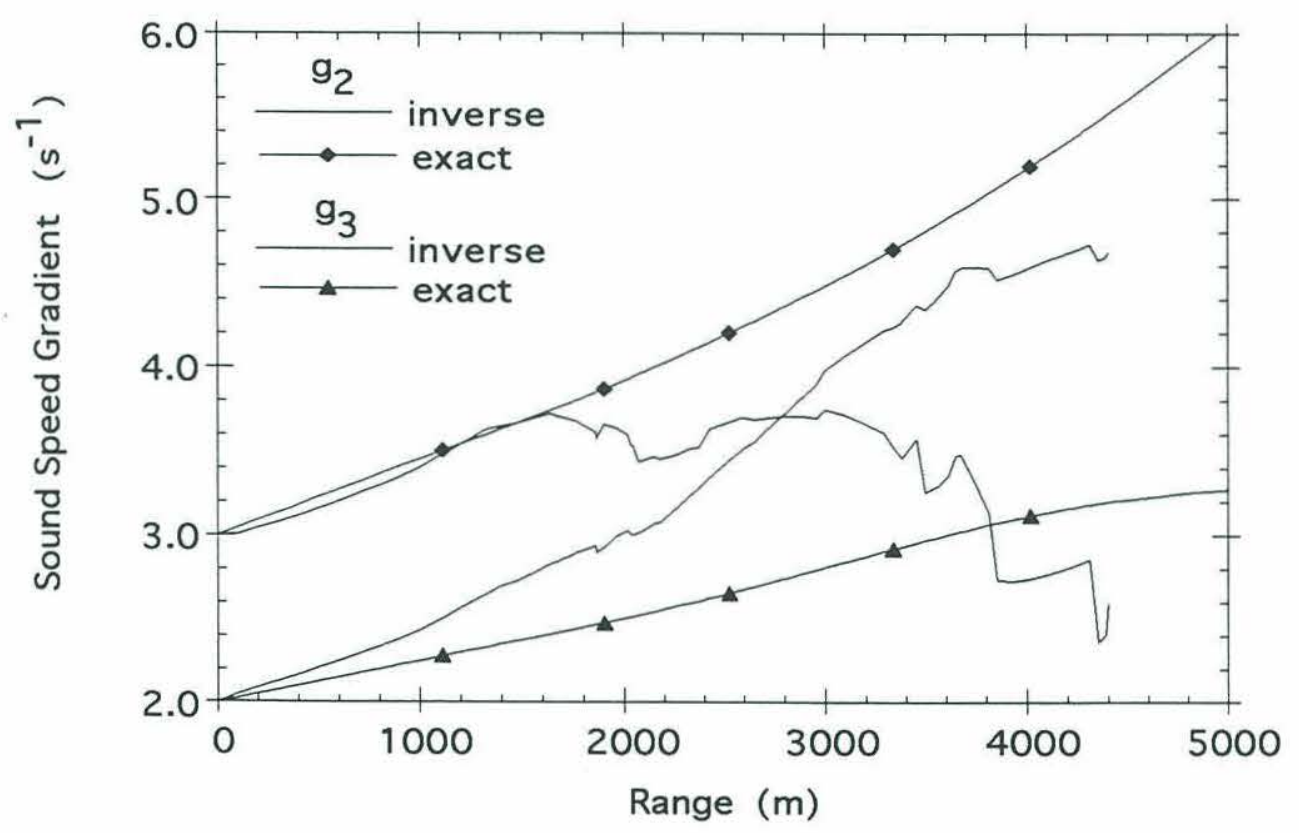

(b)

Figure 5-14: Comparison of the exact and estimated geoacoustic parameters in the shallow water model in the underdetermined case. The local eigenvalues of modes 1-4 are used in the SVD method for the estimation of the parameters. (a) Sediment interface depth $h_{3}$, (b) sound speed gradients $g_{2}$ and $g_{3}$, (c) sound speed $\tilde{c}_{2}$ at interface depth $h_{2}$, and (d) sound speed $\tilde{c}_{3}$ at interface depth $h_{3}$. 


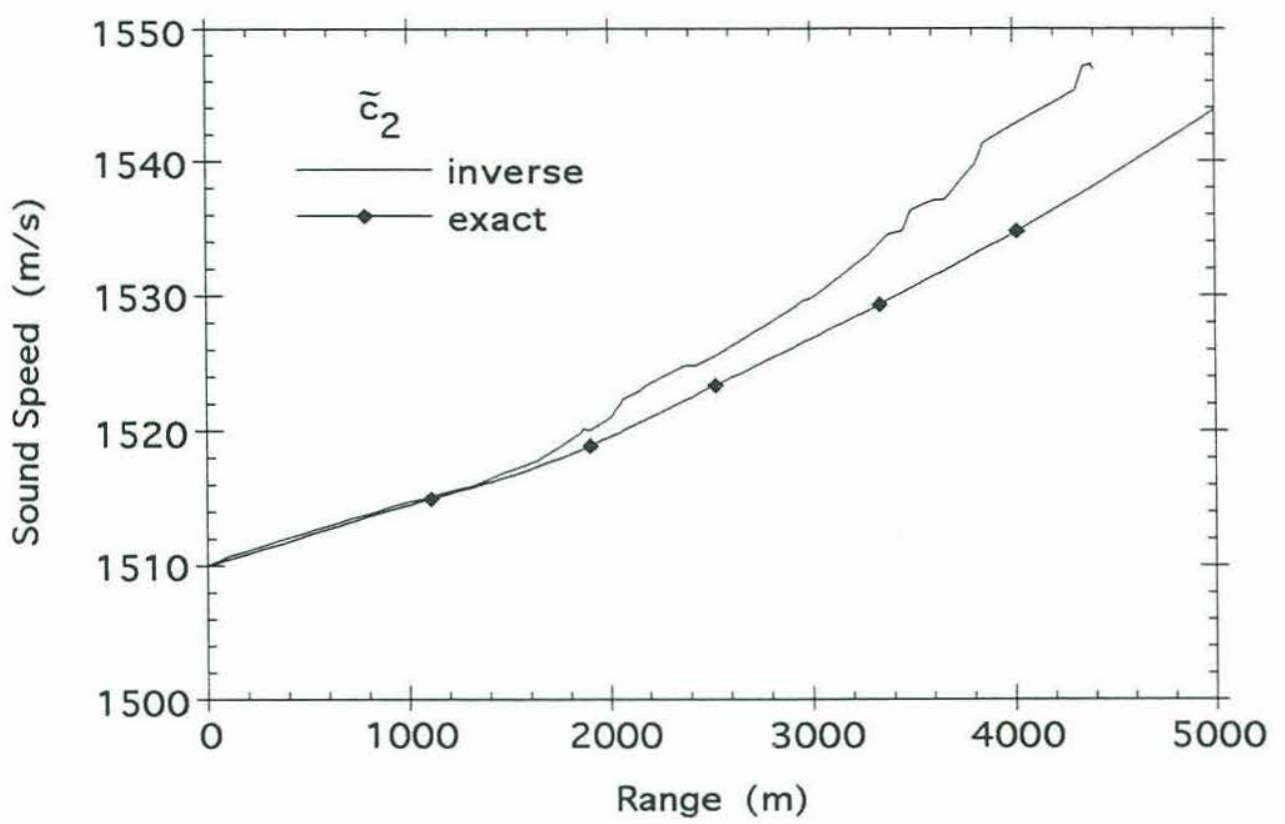

(c)

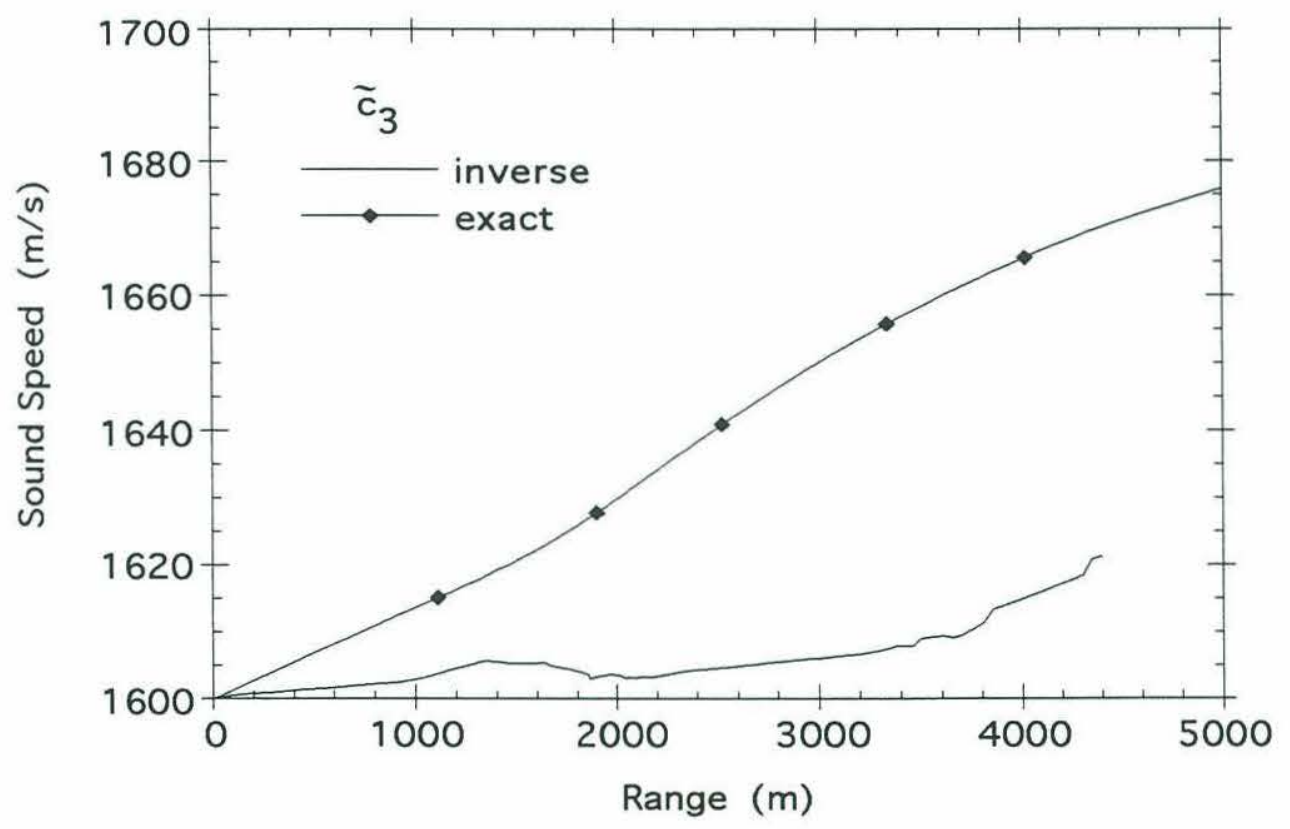

(d)

Figure 5-14, continued. 


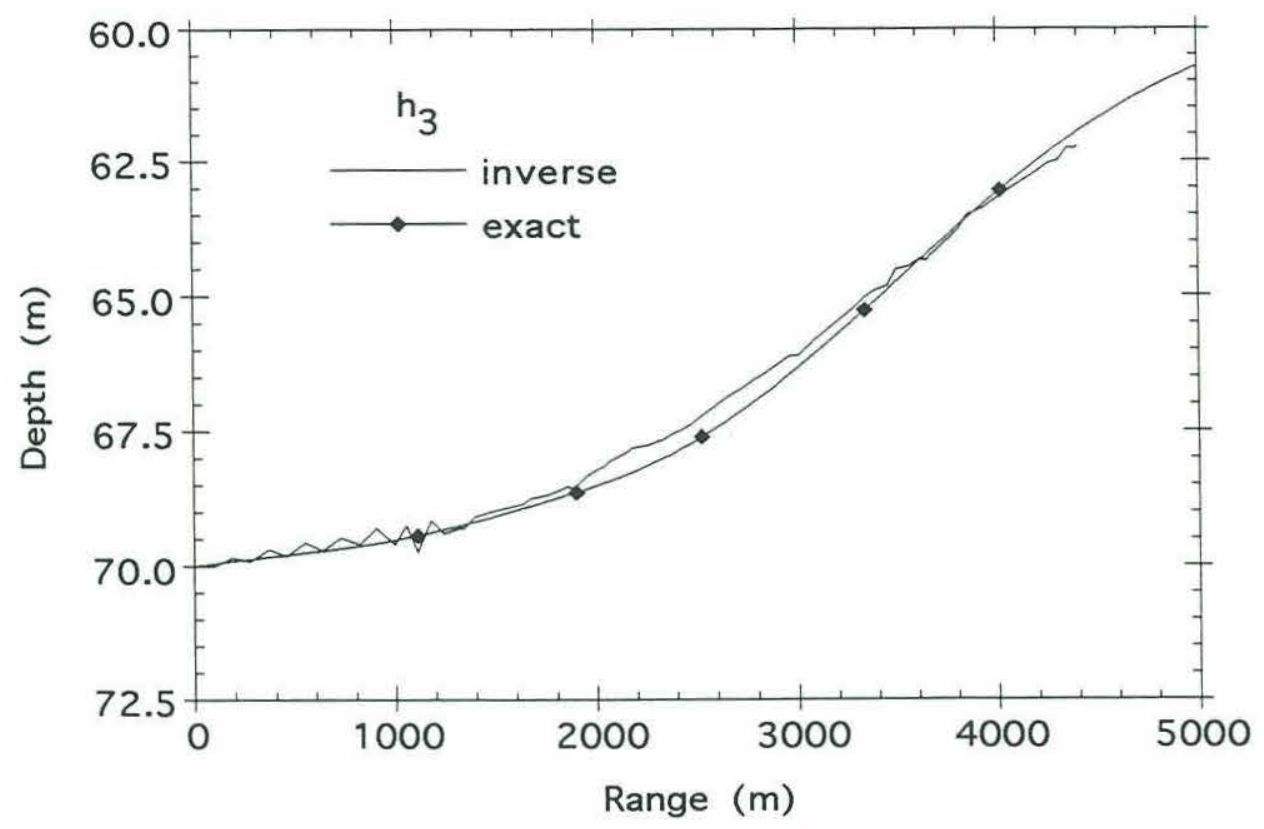

(a)

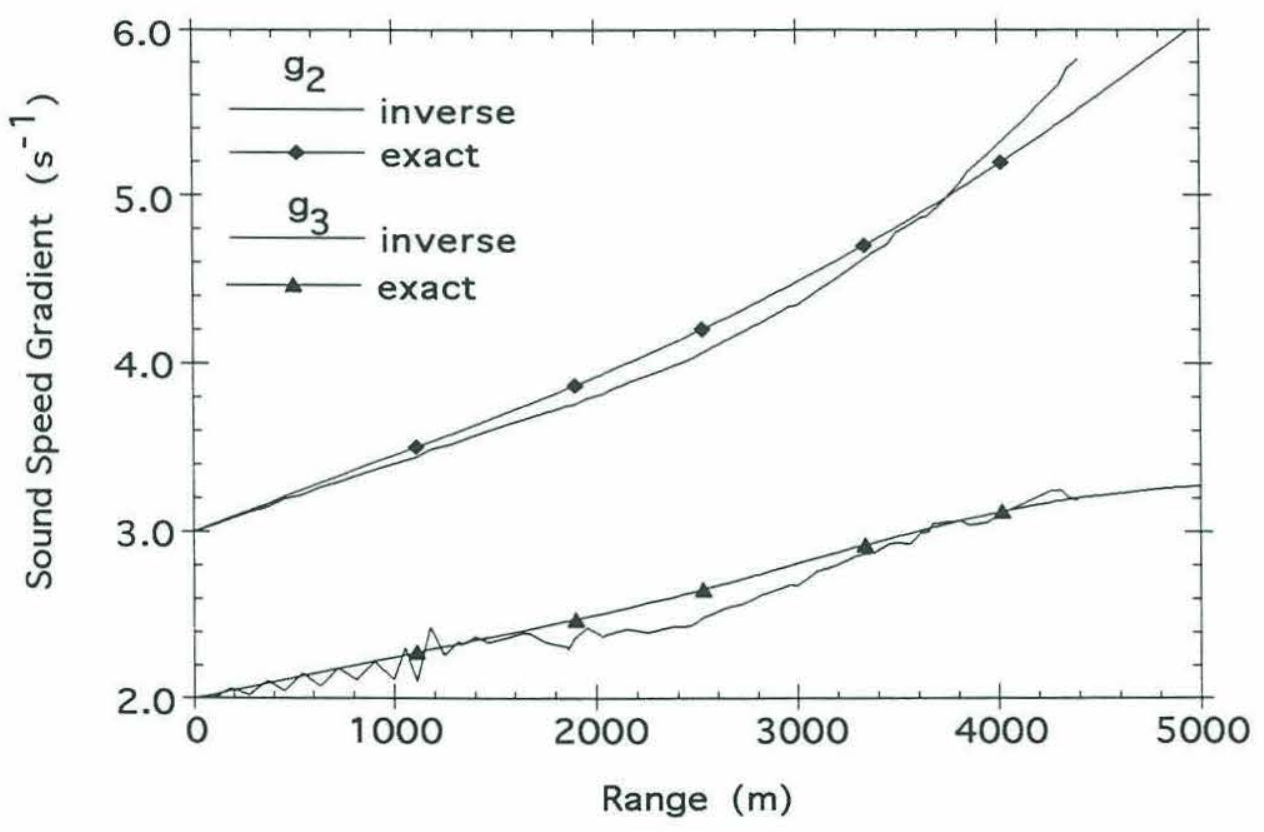

(b)

Figure 5-15: Comparison of the exact and estimated geoacoustic parameters in the shallow water model in the underdetermined case. The local eigenvalues of modes 2-5 are used in the SVD method for the estimation of the parameters. (a) Sediment interface depth $h_{3}$, (b) sound speed gra-dients $g_{2}$ and $g_{3}$, (c) sound speed $\tilde{c}_{2}$ at interface depth $h_{2}$, and (d) sound speed $\tilde{c}_{3}$ at interface depth $h_{3}$. 


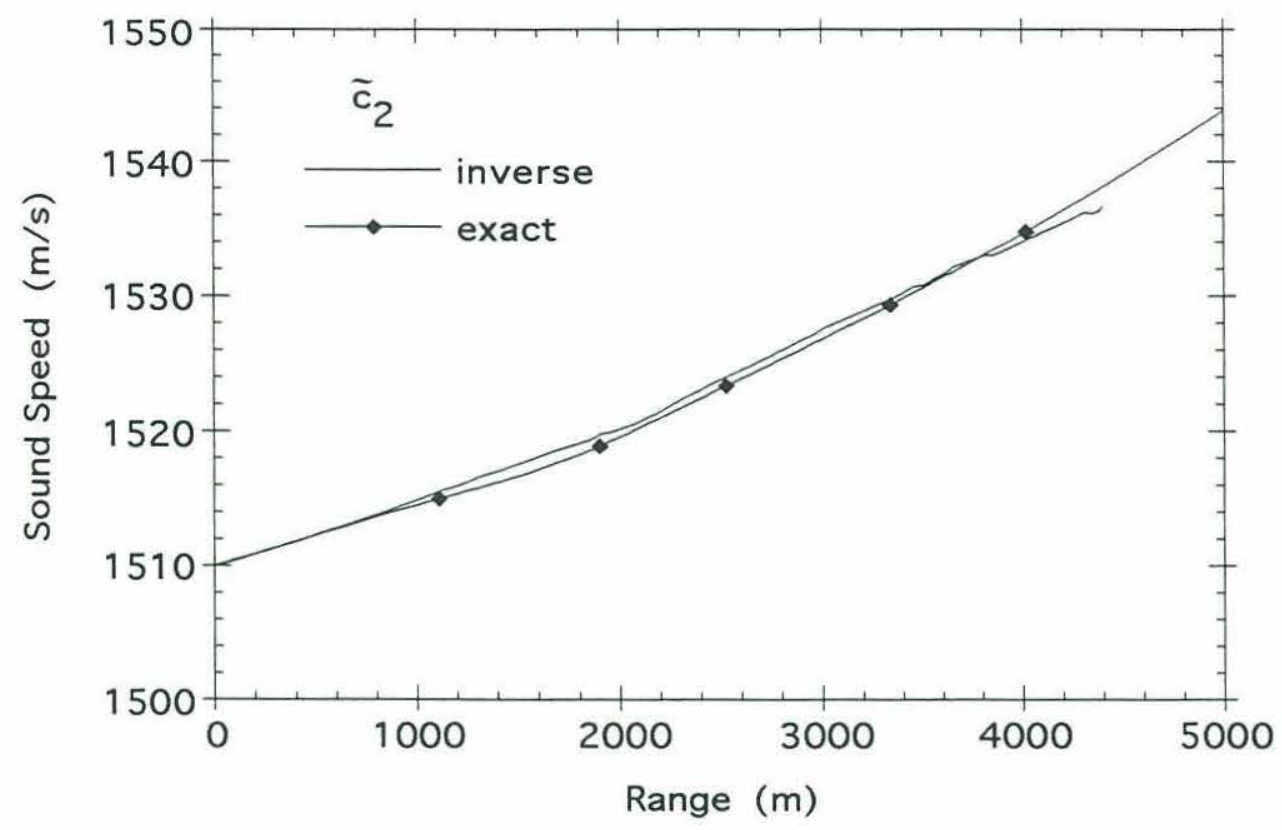

(c)

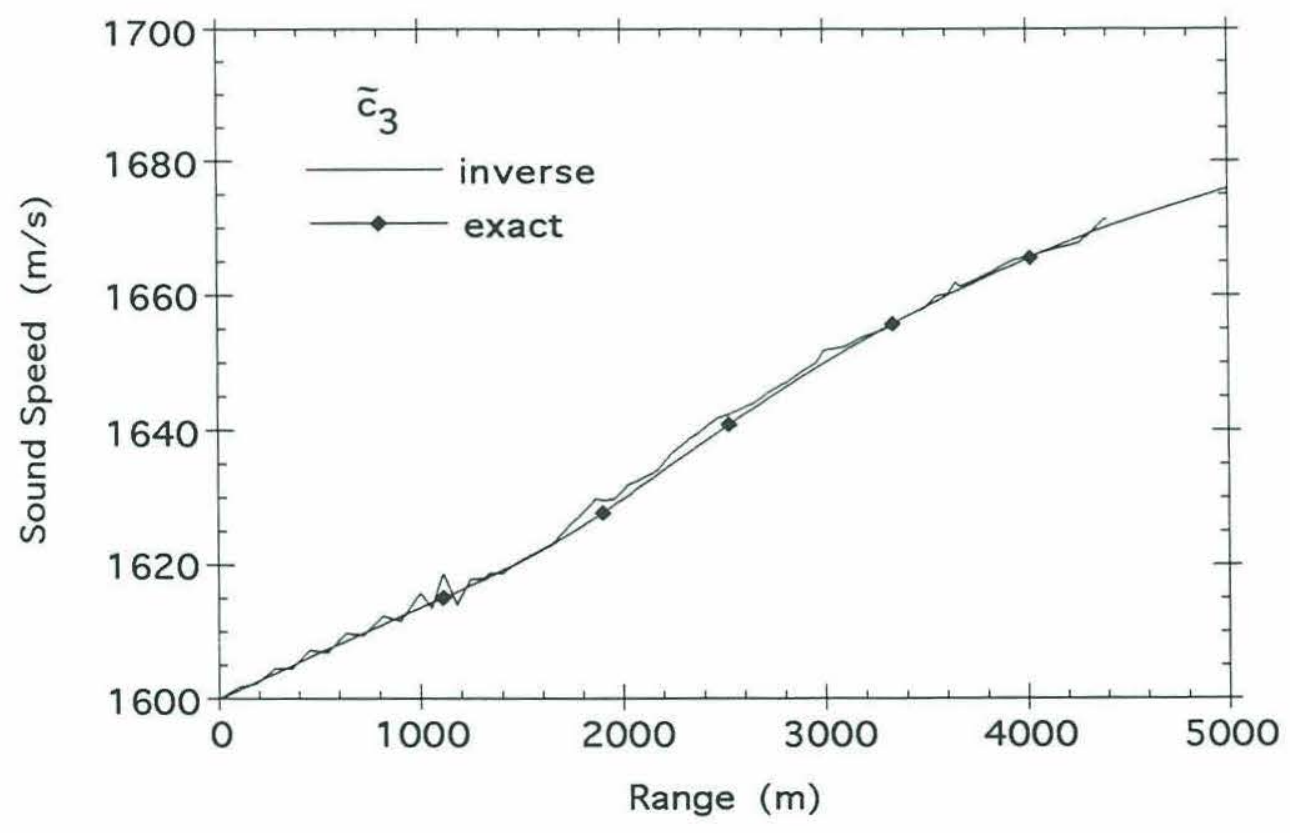

(d)

Figure 5-15, continued. 

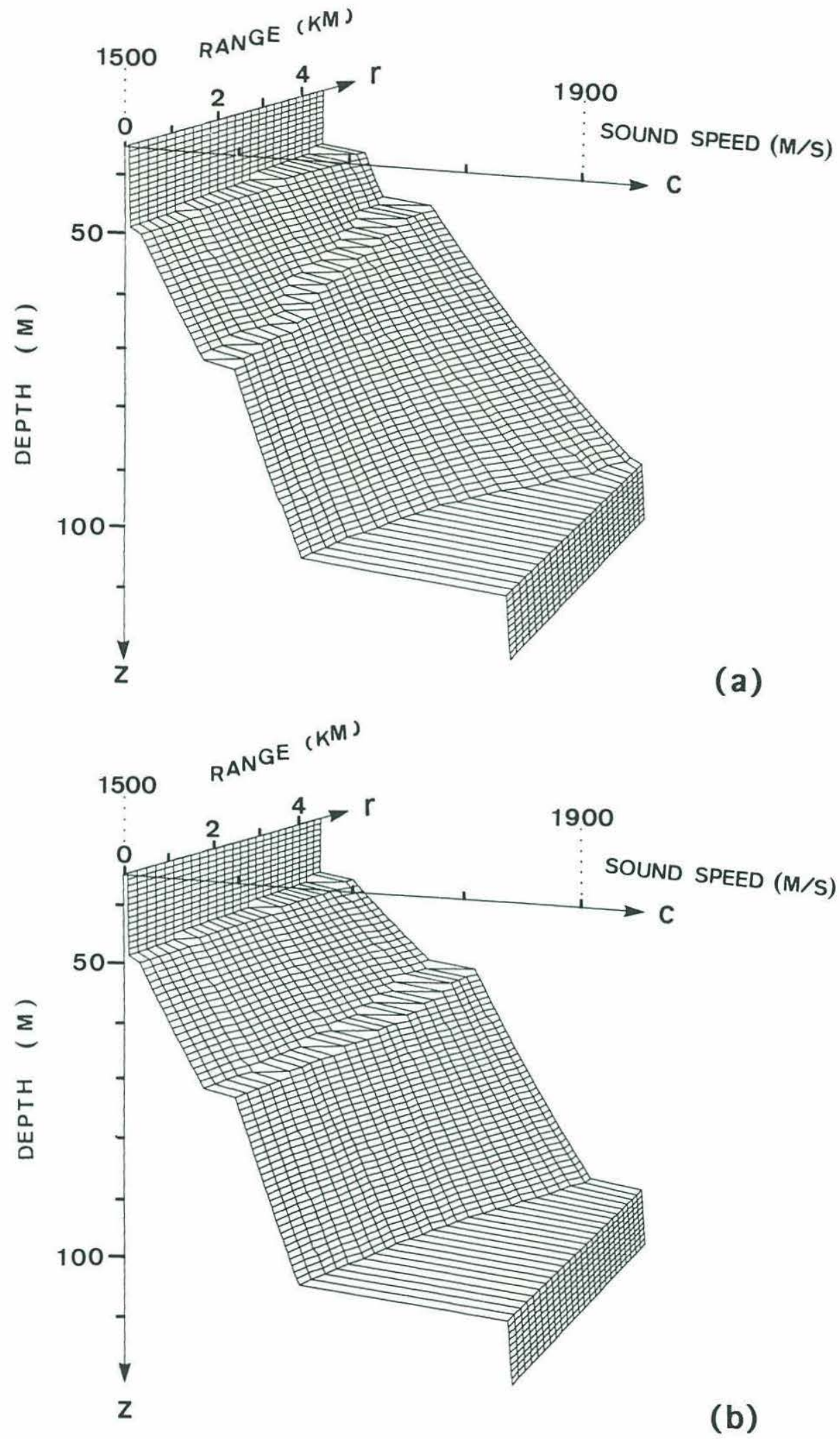

Figure 5-16: Comparison of 3-D pictures of inferred sound speed profiles in the sediment bottom in the underestimated case. (a) Modes 1-4 and (b) modes 2-5 are used as input data in the inverse method. 


\subsection{Summary}

In this chapter we explored a method for determining the local properties of the bottom sediment from the local eigenvalues; these eigenvalues were estimated by applying the Hankel transform with a sliding window to the pressure field in a horizontally as well as vertically varying waveguide.

In Section 5.1, in order to provide a basis for the development of an inverse method for determining the range-dependent geoacoustic parameters, we set up a horizontally and vertically varying, multilayered model for the bottom sediment in shallow water.

In Section 5.2, based on the bottom model set up in Section 5.1, we derived the relation between the perturbed local eigenvalues and perturbed geoacoustic parameters by utilizing linear perturbation theory.

In order to confirm the validity of the result obtained in Section 5.2, we applied it to a range-dependent Pekeris model in Section 5.3. We showed that the resulting perturbation relation from Section 5.2 yields a result which is equivalent to that obtained by directly differentiating the characteristic equation.

In Section 5.4, by representing the sound speed in each layer by an $n^{2}$-linear curve, we showed that the integral in the perturbation relation can be executed in closed form. As a result, we revealed that the variation of the local eigenvalues can be separated into terms that depend on the range variations of the sediment interface depths, the sound speed and gradient in each sediment layer, and the sound speed profile in the water column.

Based on the perturbation equation derived in Section 5.4, we developed an inversion method for determining the geoacoustic parameters in Section 5.5. We then demonstrated that we could obtain the range-dependent behavior of these parameters by solving the simultaneous perturbation equations at successive ranges. When the number of local modes is less than the number of unknown geoacoustic parameters, we showed that we can apply the singular value decomposition (SVD) method to this underdetermined problem. 
In Section 5.6, to check numerically the methods derived in this chapter, we applied them to the shallow water model used in Chapter 4.4. First, we decomposed the perturbed local eigenvalue into terms associated with the perturbed geoacoustic parameters by using the perturbation equation derived in Section 5.4. Then we determined which geoacoustic parameters have the strongest influence on the range variation of each local mode. We observed that, in this shallow water model, mode 2 is less affected by the range variation of the second sediment layer, whereas mode 5 is strongly affected by the range variation of the first and second interface depths.

Second, by assuming that these geoacoustic parameters were unknown, we tried to determine them by applying the inverse method developed in Chapter 5.5 to the compensated local eigenvalues estimated in Chapter 4.4; these eigenvalues were obtained by using the general Hankel transform with a 2-D sliding window. As a result, we showed that we can estimate accurately these unknown range-dependent parameters. Next, we used the underestimated values for the local eigenvalues as input data; these eigenvalues were obtained by using the zero-order Hankel transform with a sliding window in Chapter 4.4. As a result, we observed that the error in the estimation for the geoacoustic parameters increased; in particular, the error in the sound speed gradient of the first sediment layer is prominent. This was accounted for by the stability relation between the input and output data in the perturbed simultaneous equations. Moreover, we tried to determine the five unknown parameters by using four modes. We applied the SVD method to this underdetermined problem and consequently could obtain geoacoustic parameters close to the exact ones when we chose the modes properly (modes 2-5). It was seen that these results are related to the location of the turning point depth of each mode and its connection to the depths of the unknown parameters.

In the next chapter, we will analyze some experimental data by using the methods developed so far. 


\section{Chapter 6}

\section{Analysis of Experimental Data}

We studied the zero-order and general Hankel transform with a sliding window in Chapters 3 and 4, respectively, and also applied each transform to numerically simulated fields. In this chapter, we will analyze some experimental data by using the Hankel transform with a sliding window and estimating the local eigenvalues of the discrete modes. Then, by using these local eigenvalues, we will find a geoacoustic model whose local eigenvalues agree satisfactorily with the estimated ones. Based on this inferred model, we will examine the effect of range-dependent variation of the sediment bottom on the range variation of the local modes.

\subsection{Nantucket Sound experiment}

In this section, we will examine experimental data measured in Nantucket Sound by Frisk and his co-workers. Figure 1-1 shows the configuration of this experiment; the details can be found in Ref. 30 . The acoustic pressure due to a CW source towed away from $r=0$ to $1320 \mathrm{~m}$ was recorded by receivers at two different, fixed depths $(7.1 \mathrm{~m}$ and $12.5 \mathrm{~m}$ ). Here the $z$ axis is fixed at the receiver position as in Figure 3-8. The bathymetry observed over this range has a noticeable, but small, change at a range of about $660 \mathrm{~m}$. The sound speed in the water column is confirmed from temperature measurements to be isovelocity with $c=1503 \mathrm{~m} / \mathrm{s}$.

Since these field measurements were taken azimuthally in one direction, we cannot use the general Hankel transform with a sliding window, which requires a field with different azimuths as we discussed in Chapter 4. Here, by assuming that the field is cylindrically symmetric about the receivers, we use the zero-order Hankel transform with 
a sliding window. The output of applying the Hankel transform in Eq.(3-30) to the pressure data at $140 \mathrm{~Hz}$ is shown in Figure 6-1 in the form of $\sqrt{k_{r}}\left|g_{w}\left(k_{r}, r\right)\right|$ versus both $k_{r}$ and $r$. In this processing, a Hanning window with a $500 \mathrm{~m}$ length was applied by sliding its center position every $5 \mathrm{~m}$. Figure 6-1 indicates that two peaks associated with the first and second modes evolve with range and their peak positions in $k_{r}$ experience a relatively strong shift at about $r=500 \mathrm{~m}$. This result can account for the splitting of the spectral peaks when the Hankel transform is applied over the entire $1320 \mathrm{~m}$ aperture (see Figure 1-2). Of particular interest is the fact that the range where the peak shift occurs is different from the range of the pronounced bathymetric change ( $r=660 \mathrm{~m})$. Reference 30 assumed that the sediment bottom also changes its geoacoustic properties at $r=660 \mathrm{~m}$ and divided the waveguide into two sections at this range; the Hankel transform was then applied to each section of this waveguide in order to obtain eigenvalues for each section.

In order to examine the range of this shift further, let us elucidate the range evolution of the second mode, whose amplitude is weak compared to the first mode and is especially difficult to recognize for the near-bottom receiver. This is due to the fact that the location of the near-bottom receiver $(z=12.5 \mathrm{~m})$ is close to the null of the second mode. To enhance the spectral component associated with the second mode, the utility of mode filtering is desirable. As discussed in Chapter 3.3, knowledge about the bottom environment at the array site $(r=0 \mathrm{~m})$ is then required to execute mode filtering. This bottom environment was inferred by resorting to the iteration of forward models method. Namely, we calculated the pressure field including its phase by changing the geoacoustic parameters repeatedly until it best fit the measured pressure field near the site $(r<200 \mathrm{~m})$. By using the inferred bottom environment at the array site, the matrix $\mathbf{U}$ in Eq.(3-58) was calculated. Figure 6-2 shows the range evolution of the first and second modes after the application of mode filtering. As seen from the spectrum of Mode 2 in Figures 6-2, the first mode is not completely eliminated and prevails over the second mode at ranges where the second mode fully decays due to its stronger modal attenuation. This is partly 


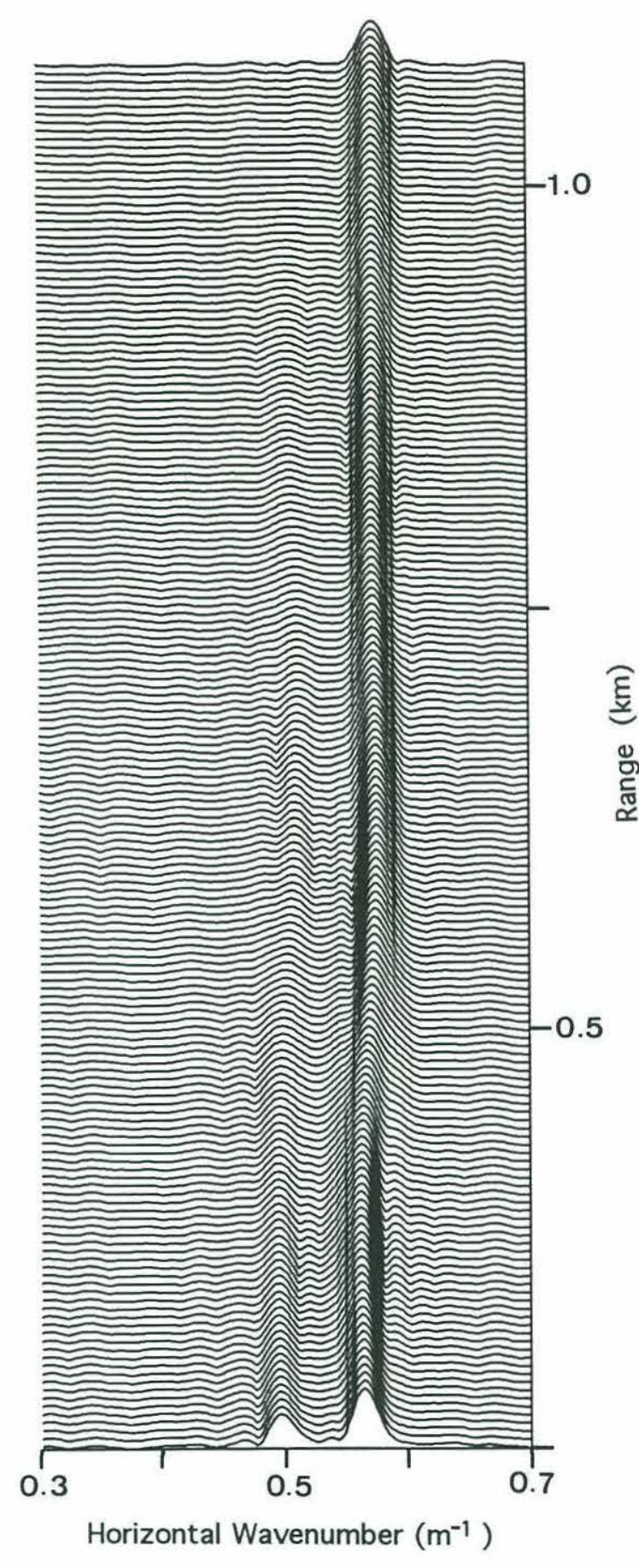

Midcolumn Receiver $(\mathrm{z}=7.1 \mathrm{~m})$

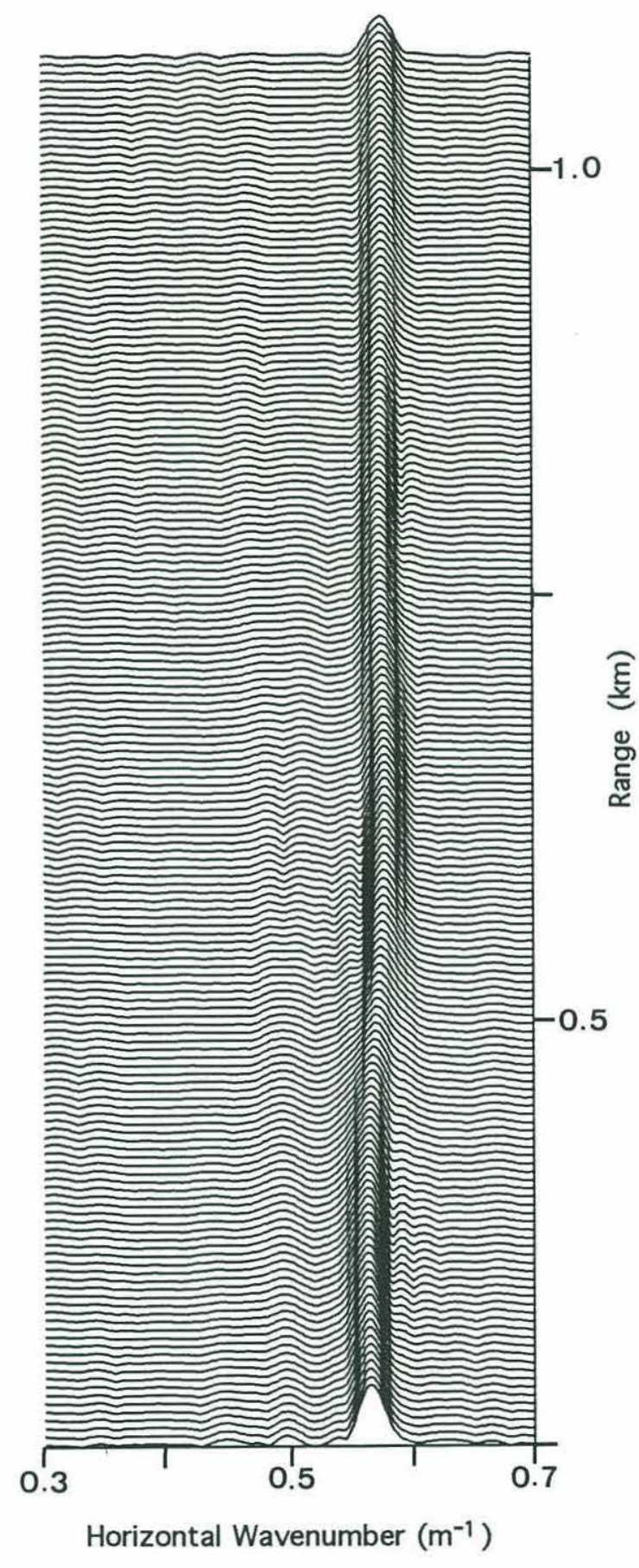

Near-bottom Receiver $(\mathrm{z}=12.5 \mathrm{~m})$

Figure 6-1: Range-dependent evolution of the modal spectrum obtained by applying the zero-order asymptotic Hankel transform with a sliding Hanning window of $500 \mathrm{~m}$ length to the Nantucket Sound data at $140 \mathrm{~Hz}$. The midcolumn receiver (left) and the nearbottom receiver (right). 


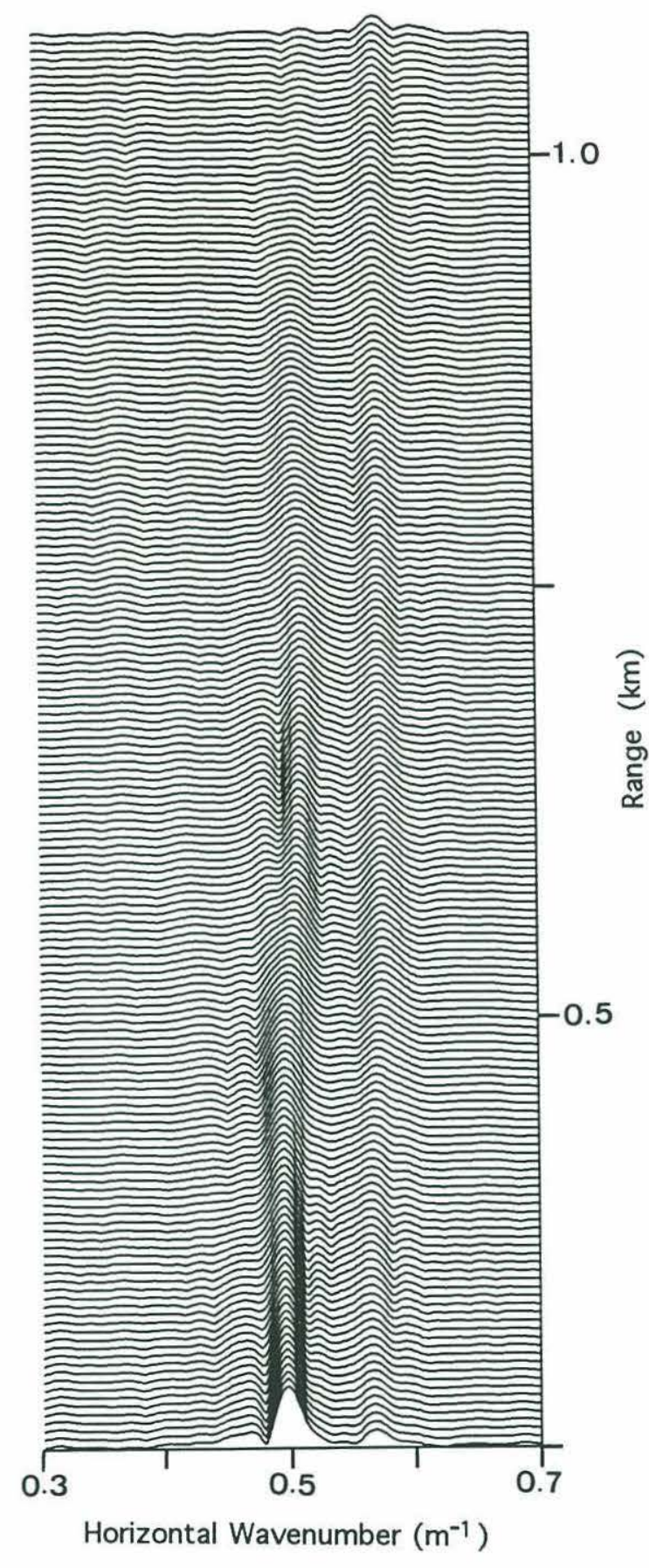

Mode 2

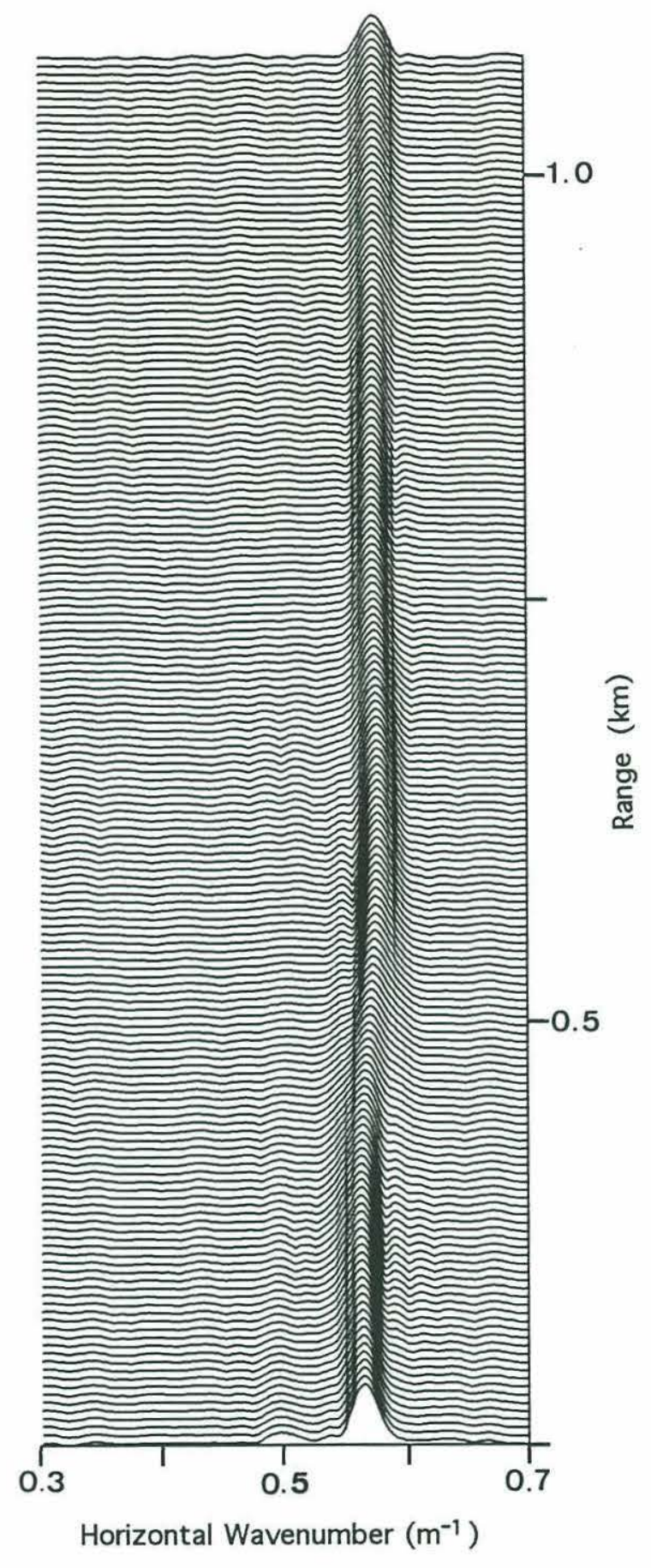

Mode 1

Figure 6-2: Range-dependent evolution of the modal spectrum obtained by applying the zero-order asymptotic Hankel transform with a sliding Hanning window of $500 \mathrm{~m}$ length to the mode-filtered Nantucket Sound data at $140 \mathrm{~Hz}$. Mode 1 (right) and mode 2 (left). 
because of the error in the estimation of the bottom properties at the array site and also partly because of inadequate synchronization in the phases measured at the two receivers. From the enhanced, mode-filtered amplitude of the second mode, however, we can confirm that the peak shift of the second mode also occurs at a range less than $660 \mathrm{~m}$.

Figure 6-3 shows the peak positions of the first and second modes from each spectrum in Figure 6-2; these peak positions are also compared with those obtained by applying the same processing to the simulated pressure field. This field was generated using adiabatic mode theory (Eq.(2-12)) with a geoacoustic model having the $n^{2}$-linear profiles shown in Figures 6-4(a)-(d). This model was inferred by trial and error, i.e., by changing the values of the geoacoustic parameters until the measured and computed modal peak trajectories produced the level of agreement shown in Figure 6-3. By comparing the inferred sound speed and its gradient with the results of Hamilton [84], we assumed that the bottom consists of silt and sand layers. This geoacoustic model was also checked by comparing the measured and simulated pressure fields. Figures 6-5(a) and (b) show the comparison of the relative amplitudes and modulated phases, respectively.

Since the sound speed gradient of sand takes on a large value near the water-bottom interface and decreases rapidly with depth [84], the sound speed profile in the sandy portion was partitioned into four layers having different gradients as shown in Figures 64(c) and (d). Note that the gradient and the sound speed at each sand layer depth are not independent of each other in order to satisfy the condition of continuous sound speed across the layer interfaces. In spite of this, we have a total of thirteen unknown parameters and so we could not effectively use the inverse methods from Chapter 5.5. As indicated in Figure 6-4, however, we were able to estimate that the geoacoustic properties in the sediment change significantly at about $r=500 \mathrm{~m}$ rather than $r=660 \mathrm{~m}$.

At this point, by using the inferred geoacoustic model, let us examine the effect of the error that has originated from the finite length of the window in the Hankel transform. 


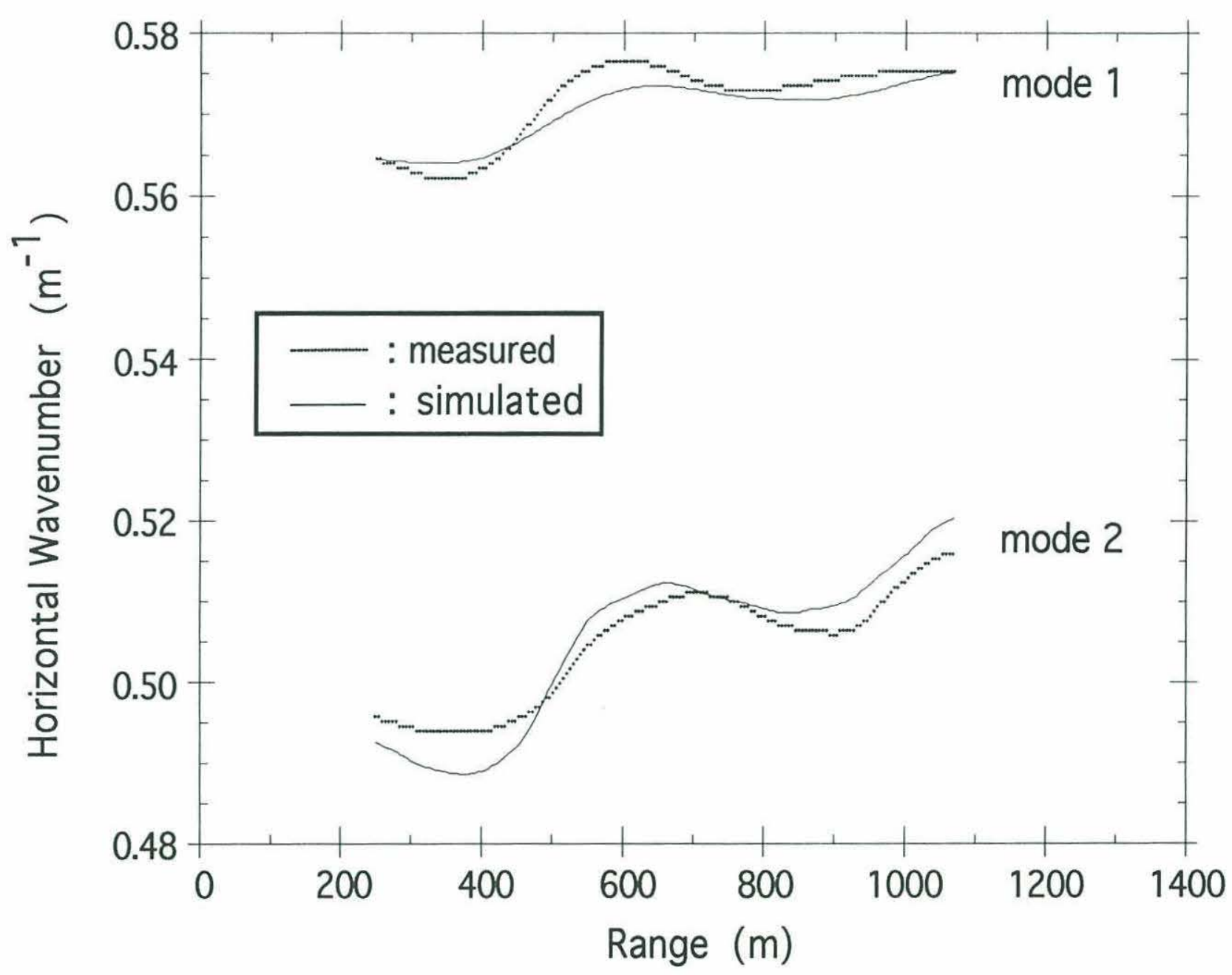

Figure 6-3: Comparison of modal peak trajectories measured in Nantucket Sound and computed using the bottom environment shown in Figure 6-4. 


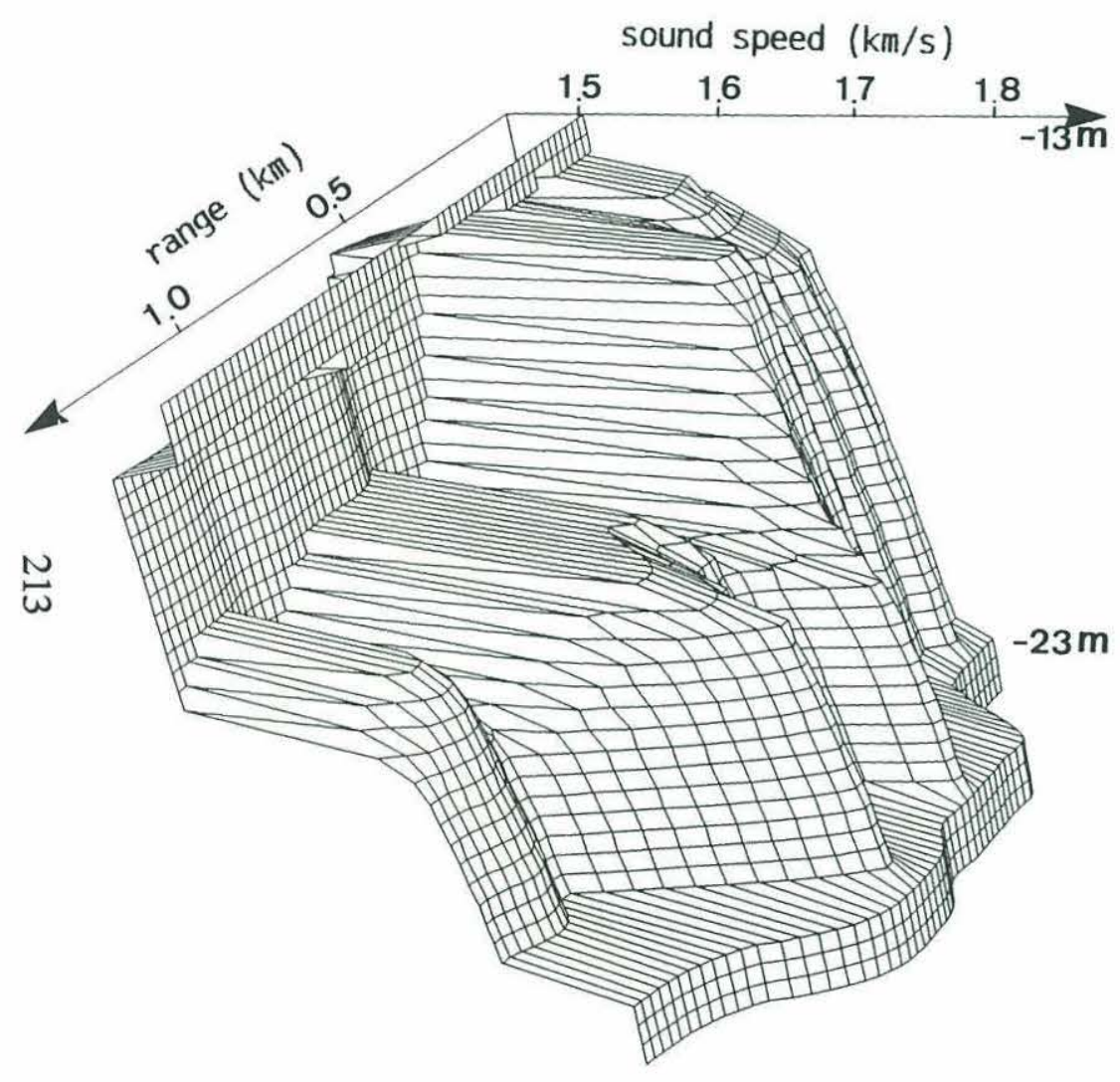

(a)

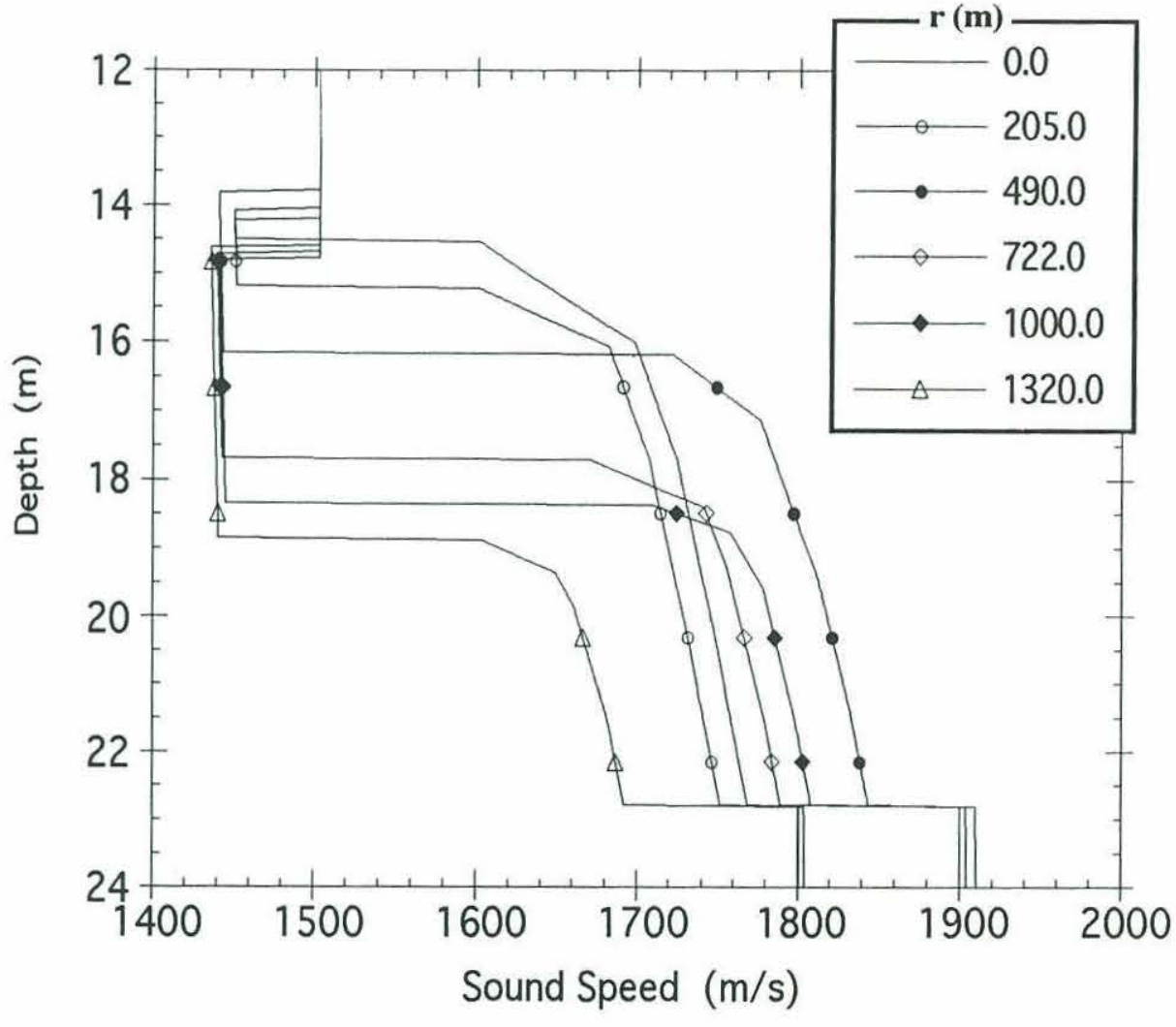

(b)

Figure 6-4: Bottom environment inferred from a comparison of the peak trajectories in the modal spectra for the experimental and simulated fields in Figure 6-3. (a) Range and depth dependence of the sediment sound speed, (b) sediment sound speed profiles at different ranges, (c) sediment layer structure, and (d) lateral variation of the sound speed gradient in each sediment layer. 


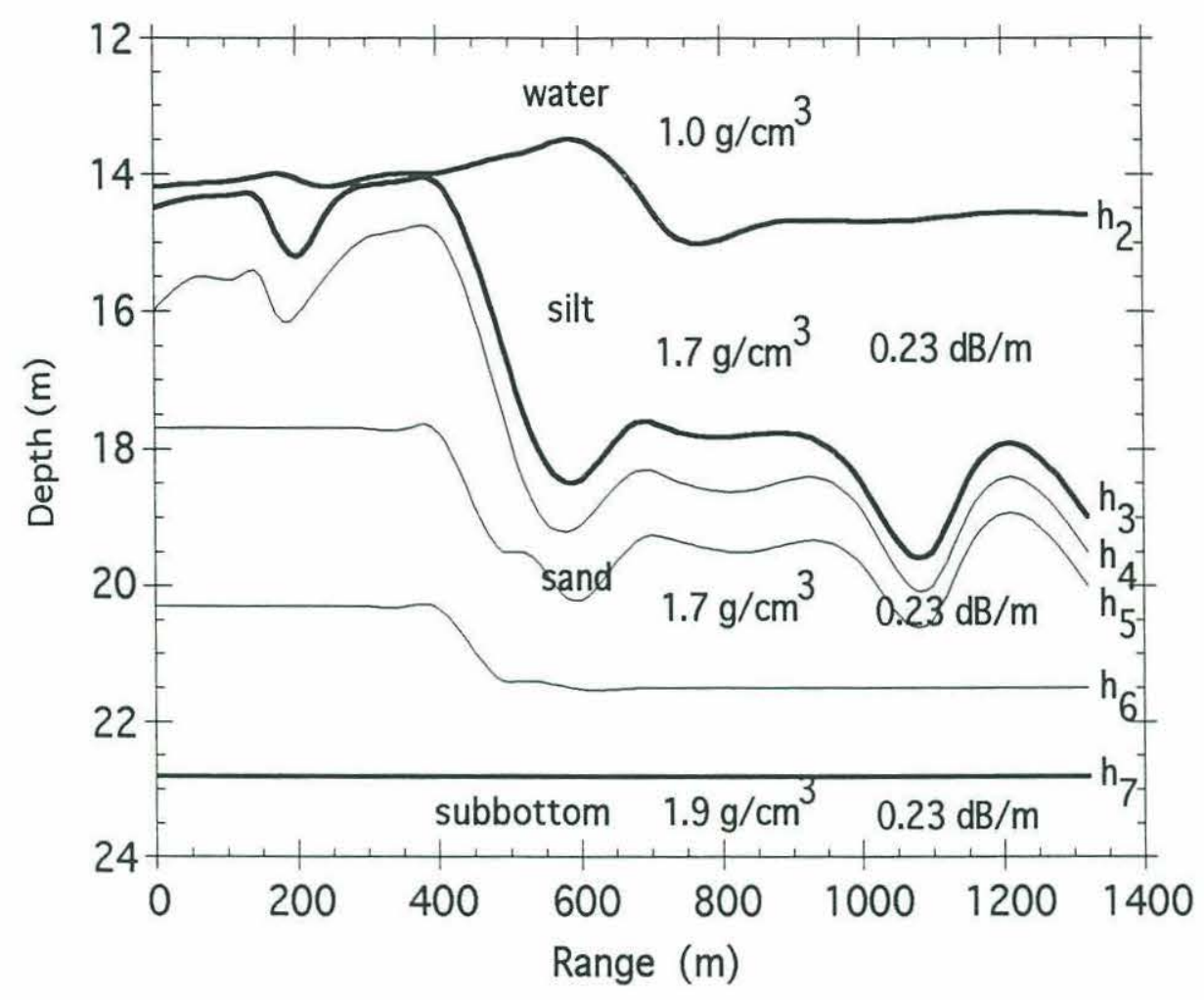

(c)

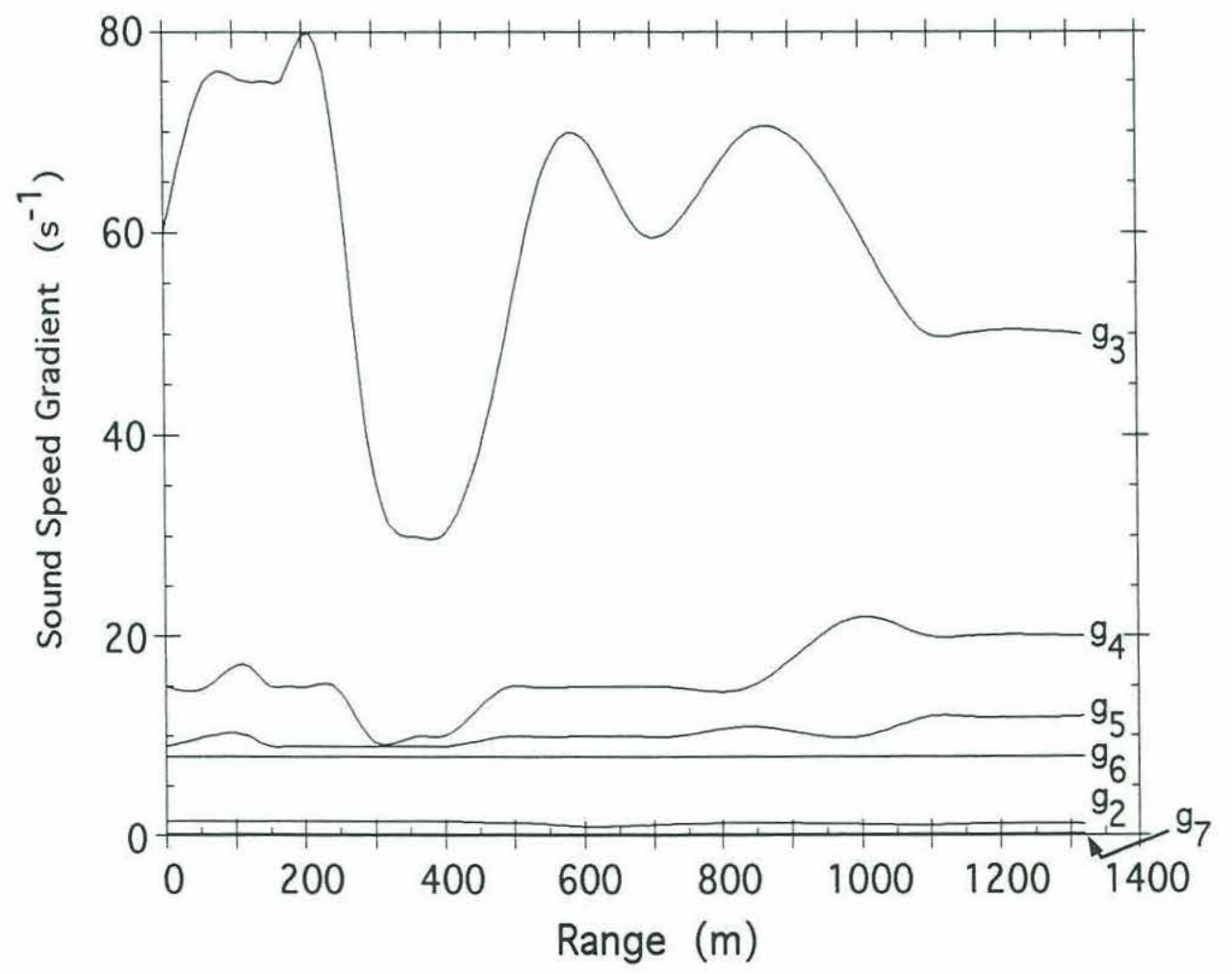

Figure 6-4, continued. 


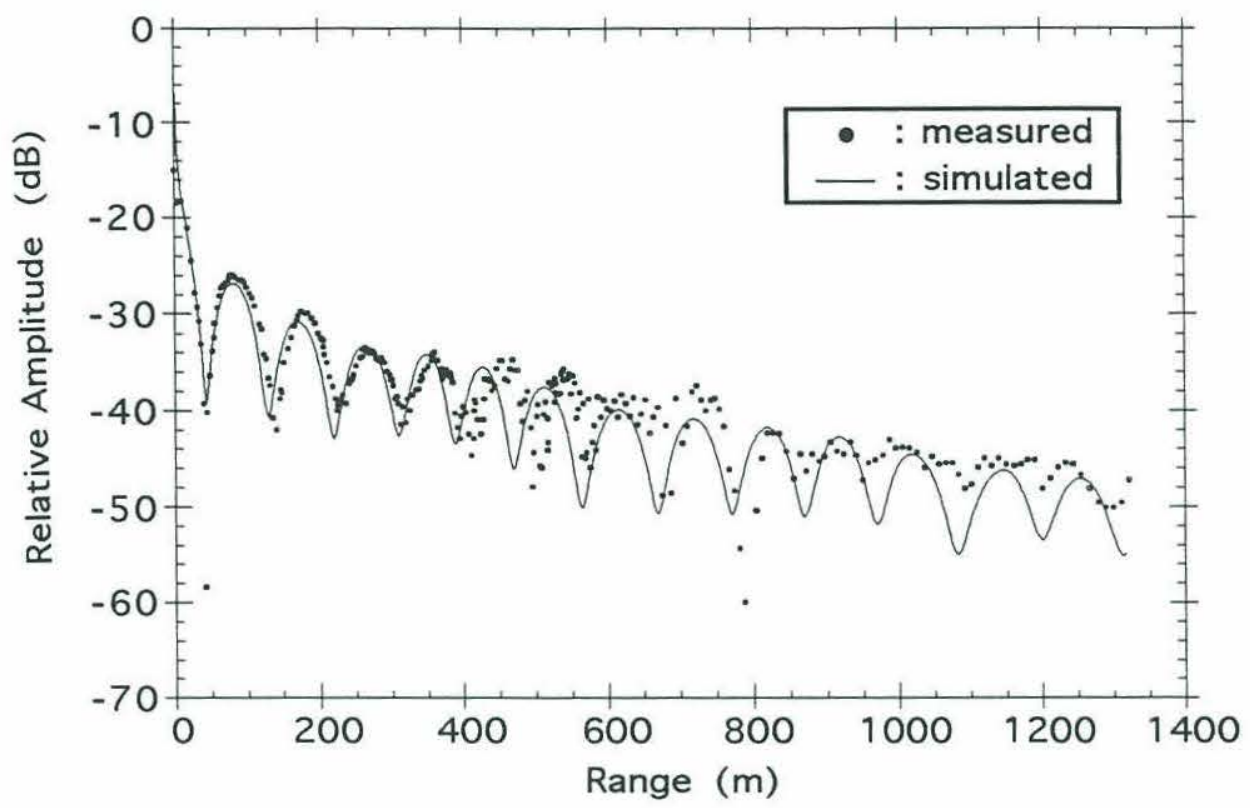

Midcolumn Receiver $(z=7.1 \mathrm{~m})$

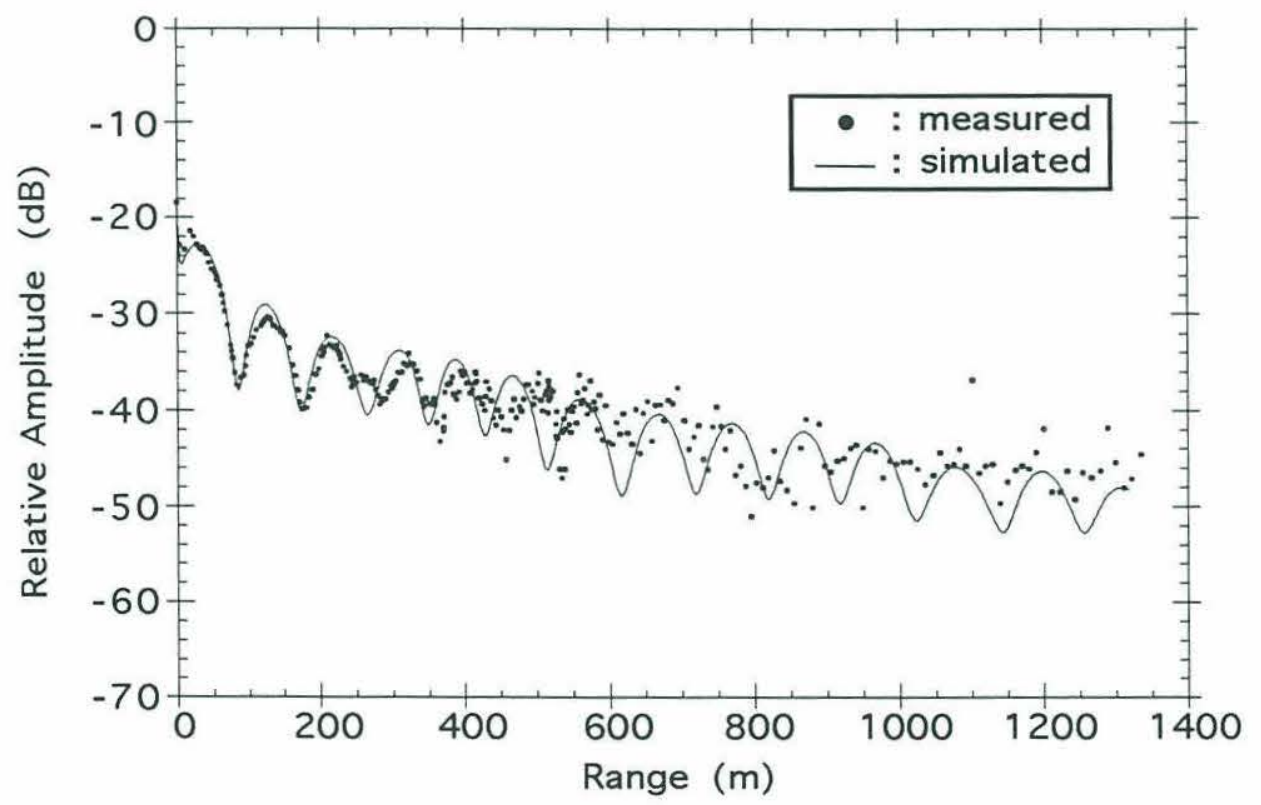

Near-bottom Receiver $(z=12.5 \mathrm{~m})$

Figure 6-5: Comparison of the measured and simulated pressure fields. (a) Relative amplitude and (b) modulated phase. Both phases in (b) are modulated as $\Phi(r) \rightarrow \Phi(r)-k_{0} r$ with $k_{0}=\omega / c_{0}$ and $c_{0}=1550 \mathrm{~m} / \mathrm{s}$. 


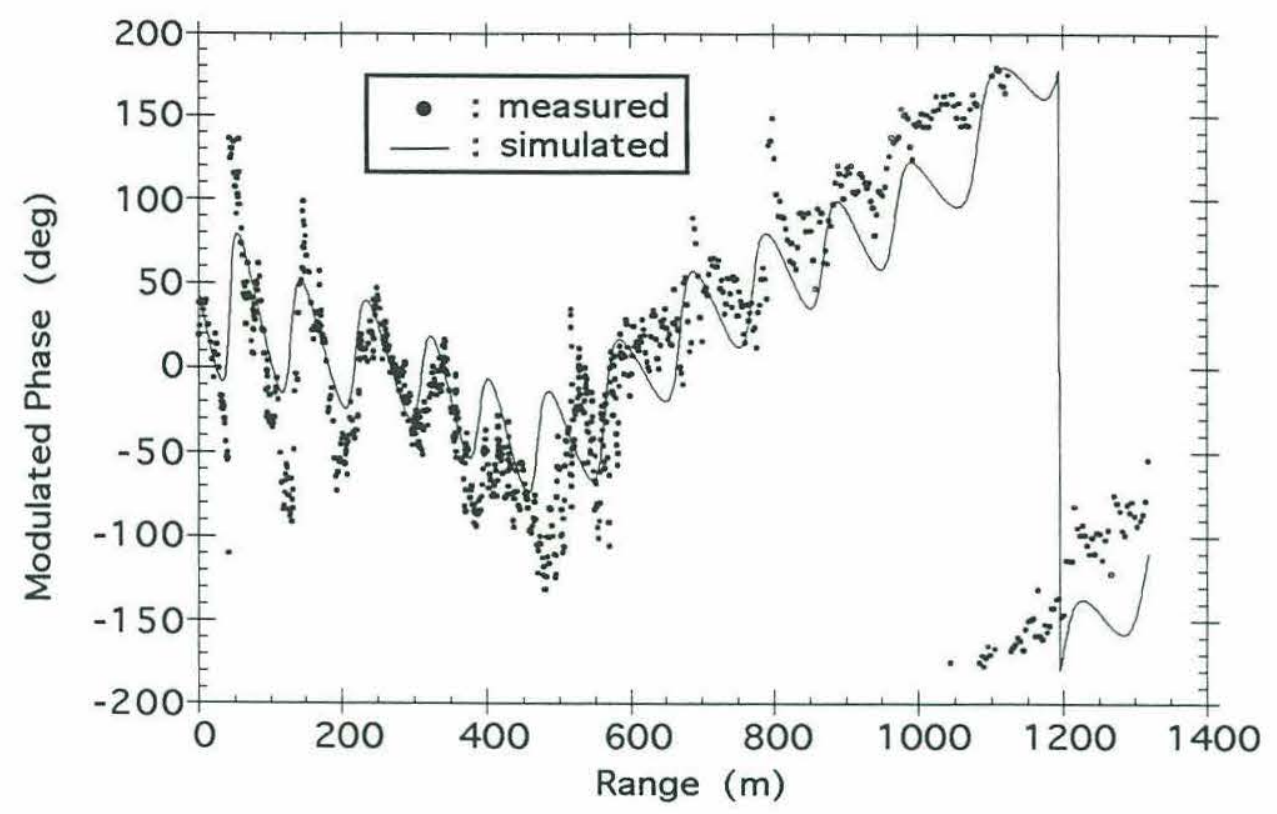

Midcolumn Receiver $(z=7.1 \mathrm{~m})$

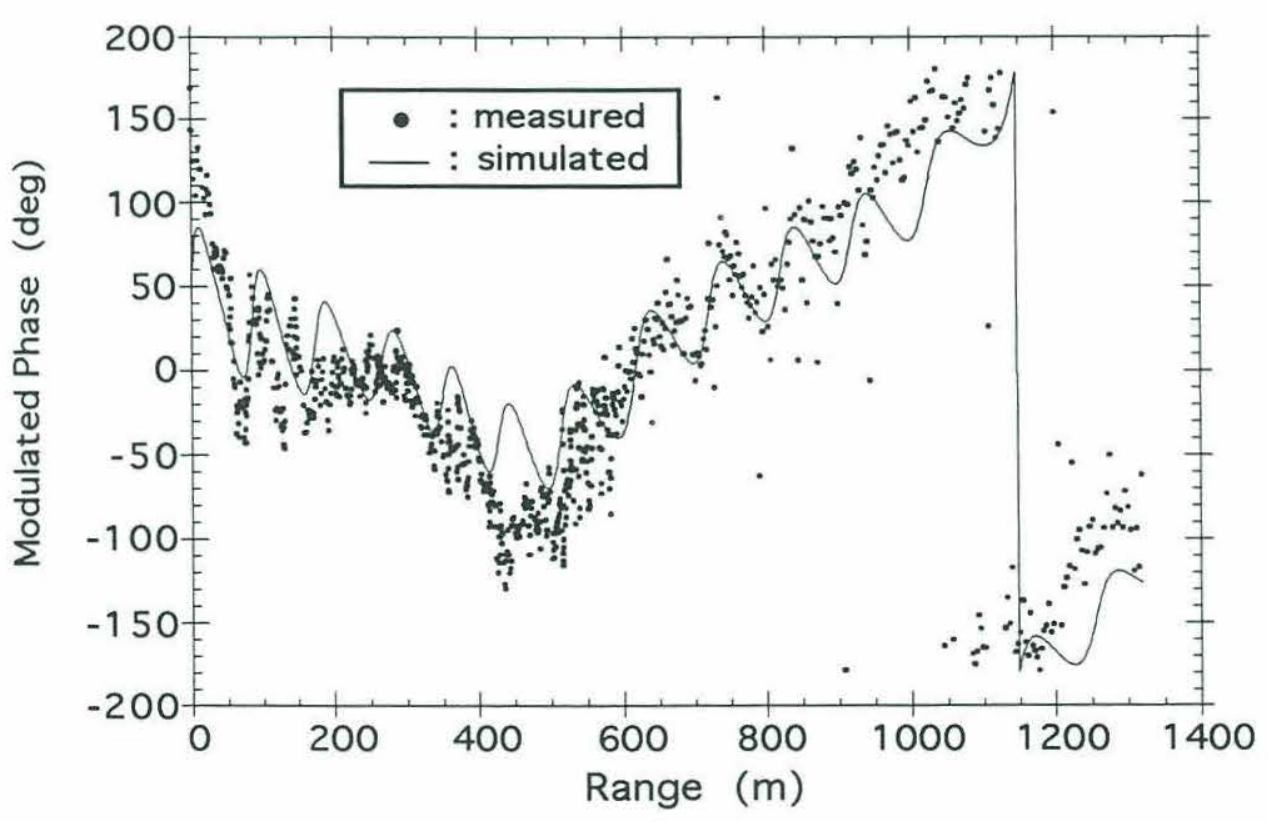

Near-bottom Receiver $(z=12.5 \mathrm{~m})$

Figure 6-5, continued. 
In Figure 6-6, we compare the modal peak trajectories obtained by using the $100 \mathrm{~m}$ and $500 \mathrm{~m}$ length Hanning windows after the application of mode filtering; in this figure, the exact eigenvalues are also shown. As also detailed in Chapter 3.2, the peak position obtained using the $500 \mathrm{~m}$ length window has a larger departure than that obtained using the $100 \mathrm{~m}$ length window at ranges where $\left|d^{2} \kappa_{n} / d r^{2}\right|$ is large; we can observe, however, that both trajectories have a relatively strong shift at about the same range $(r=500 \mathrm{~m})$. Therefore, from this result, we can see that the finite length of the window is not associated with the difference between the two ranges $(500 \mathrm{~m}$ and $660 \mathrm{~m})$.

Finally, based on the inferred bottom model in Figure 6-4, we may study the influence of the lateral changes in bottom properties on the range-dependent evolution of the modal eigenvalues by using the perturbation relation in Eq.(5-76). Figure 6-7 shows that $\Delta \kappa_{n}$ is decomposed into terms associated with the variation in bathymetry, the depth of the sediment interface between the silt and the sand layers, and the sound speed in the silt and sand layers. From these results, it is seen that the lateral change in the interface between the silt and the sand layers contributes most significantly to the range variation of the local eigenvalues in this inferred model. 


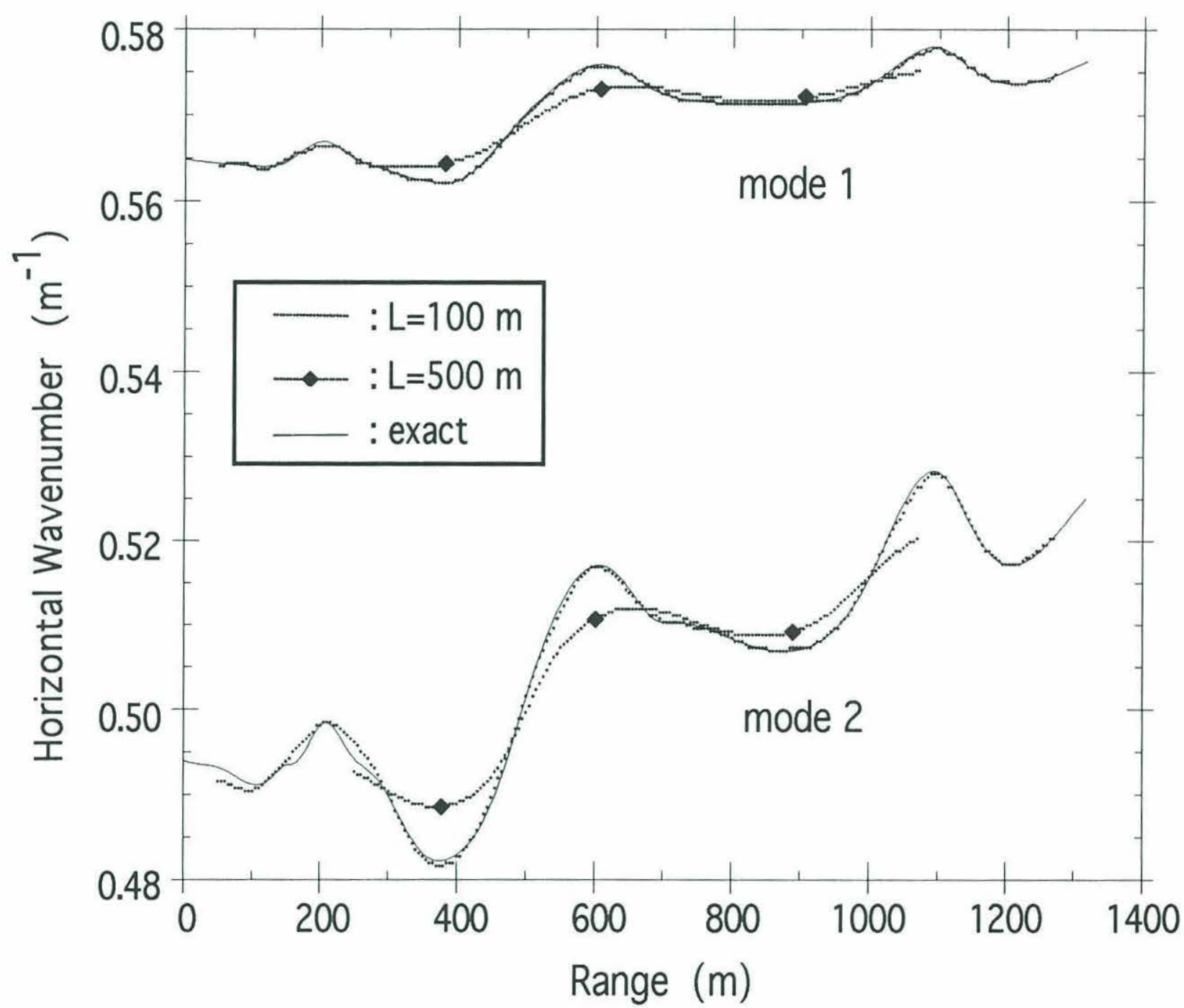

Figure 6-6: Comparison of the modal peak trajectories resulting from applying different length (L) Hanning windows to the simulated field data. The exact local eigenvalues are also shown. 


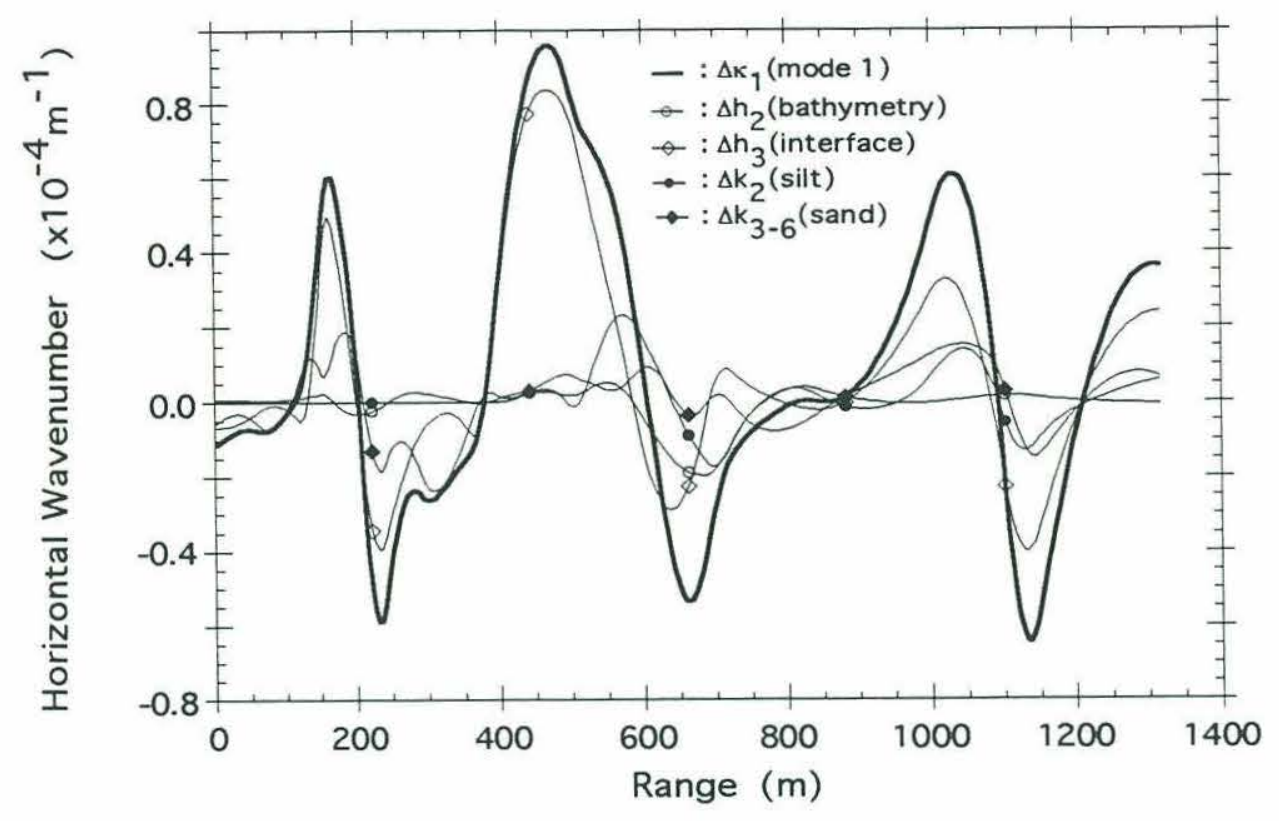

(a)

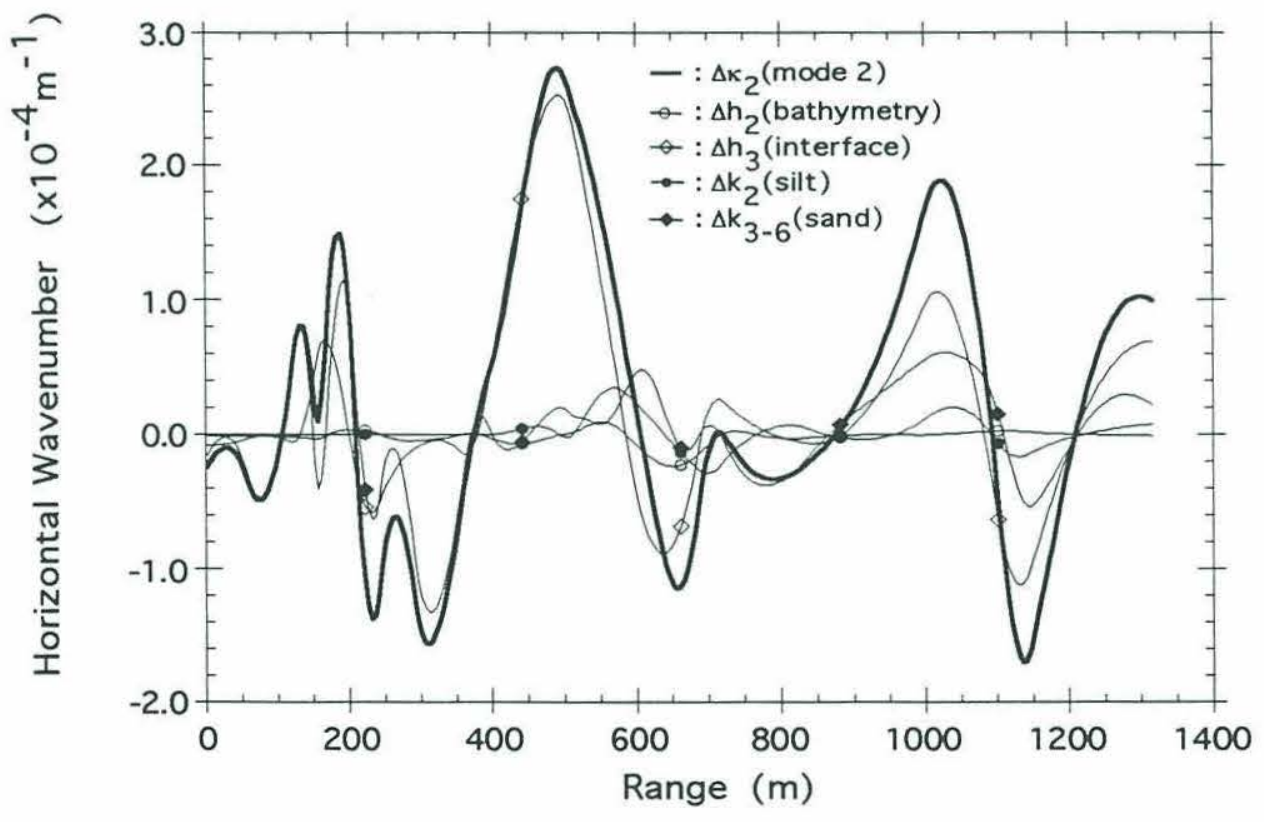

(b)

Figure 6-7: Decomposition of perturbed local eigenvalues into the terms associated with the perturbed geoacoustic parameters. (a) The first mode and (b) the second mode of the simulated field data. 


\subsection{Hudson Canyon experiment}

In this section we will analyze a subset of experimental data measured in Hudson Canyon on the New Jersey continental shelf (Figure 6-8) by Carey and his co-workers $[85,86]$. In this experiment the field measurements were carried out with a vertical array of 24 equally spaced hydrophones as shown in Figure 6-9. Here we focus on the separation of modes by using mode filtering applied to the output of the vertical array.

The field data we chose to analyze here are the acoustic pressure due to a CW source of $50 \mathrm{~Hz}$, which was towed from about $r=4000 \mathrm{~m}$ to $100 \mathrm{~m}$ along a track parallel to the shelf (TL2). This track was designed to have a uniform water depth. The bathymetry was measured using a UQN-15 fathometer and the water depth along this track has a mean value of $72.0 \mathrm{~m}$. The sound speed in the water column was also obtained by using both a Sound Velocity Profiler (SVP-16) and a Conductivity Temperature and Depth (CTD) system: the former measures sound speed directly, whereas the latter measures conductivity and temperature from which sound speed is calculated. Figure 6-10 shows the SVP and CTD sound speed profiles for the TL2 run.

The wavenumber spectrum can be obtained by applying the zero-order asymptotic Hankel transform in Eq.(3-26) to this pressure field over the entire 4000-m range. In this transform we can use a rectangular window (Eq.(3-25)) to truncate the pressure field at $4000 \mathrm{~m}$ and no weight is employed in order to handle equally the wavenumber components at each range. Figure 6-11(a) shows the wavenumber spectrum obtained by using receiver $\mathrm{H} 18$ ( $\mathrm{z}=57.5 \mathrm{~m})$. Each spectrum has a different shape, depending on the receiver depth as shown in Figure 6-12(a), since the eigenfunction of each mode varies with depth. These spectra, however, have peaks at the same positions in wavenumber. Figure 6-11(b) shows the spectrum averaged for all receivers and Table 6-1 lists the peak positions in this spectrum.

If the sediment properties are range-independent along the track TL2 and horizontal refraction is small enough to be negligible, then we can determine the eigenvalues of the 
normal modes from the peak positions in the average spectrum. From the narrow bandwidth around the spectral peaks of the first and second modes, we can expect that the sediment near the water-bottom interface is almost horizontally stratified. On the other hand, it is observed in Figure 6-12(a) that for the receivers H17 through H24 the spectral peak of the fourth mode is split. From this result only, we cannot determine if the split is due to range-dependence in the lower portion of sediment or due to other effects such as noise interference.

Based on the assumption that the medium along the track TL2 is range-independent, the sound speed in the bottom can be inferred by applying a perturbative inverse method [Rajan, 50] to the peak positions in Table 6-1. The dots in Figure 6-13 show the sound speed obtained by Rajan using this technique. The segmented lines in the same figure represent a sequence of $n^{2}$-linear fits to this profile.

Table 6-1: Peak positions of the spectrum in Figure 6-11 and mode eigenvalues of the simulated field.

$\begin{array}{ccc}\text { mode } & \begin{array}{c}\text { peak position }\left(\mathrm{m}^{-1}\right) \\ \text { (measure field) }\end{array} & \begin{array}{c}\text { eigenvalue }\left(\mathrm{m}^{-1}\right) \\ \text { (simulated field) }\end{array} \\ 1 & 0.2086 & 0.2086 \\ 2 & 0.1992 & 0.1992 \\ 3 & 0.1852 & 0.1850 \\ 4 & 0.1735 & 0.1735 \\ 5 & 0.1634 & 0.1628 \\ 6 & 0.1518 & 0.1513\end{array}$




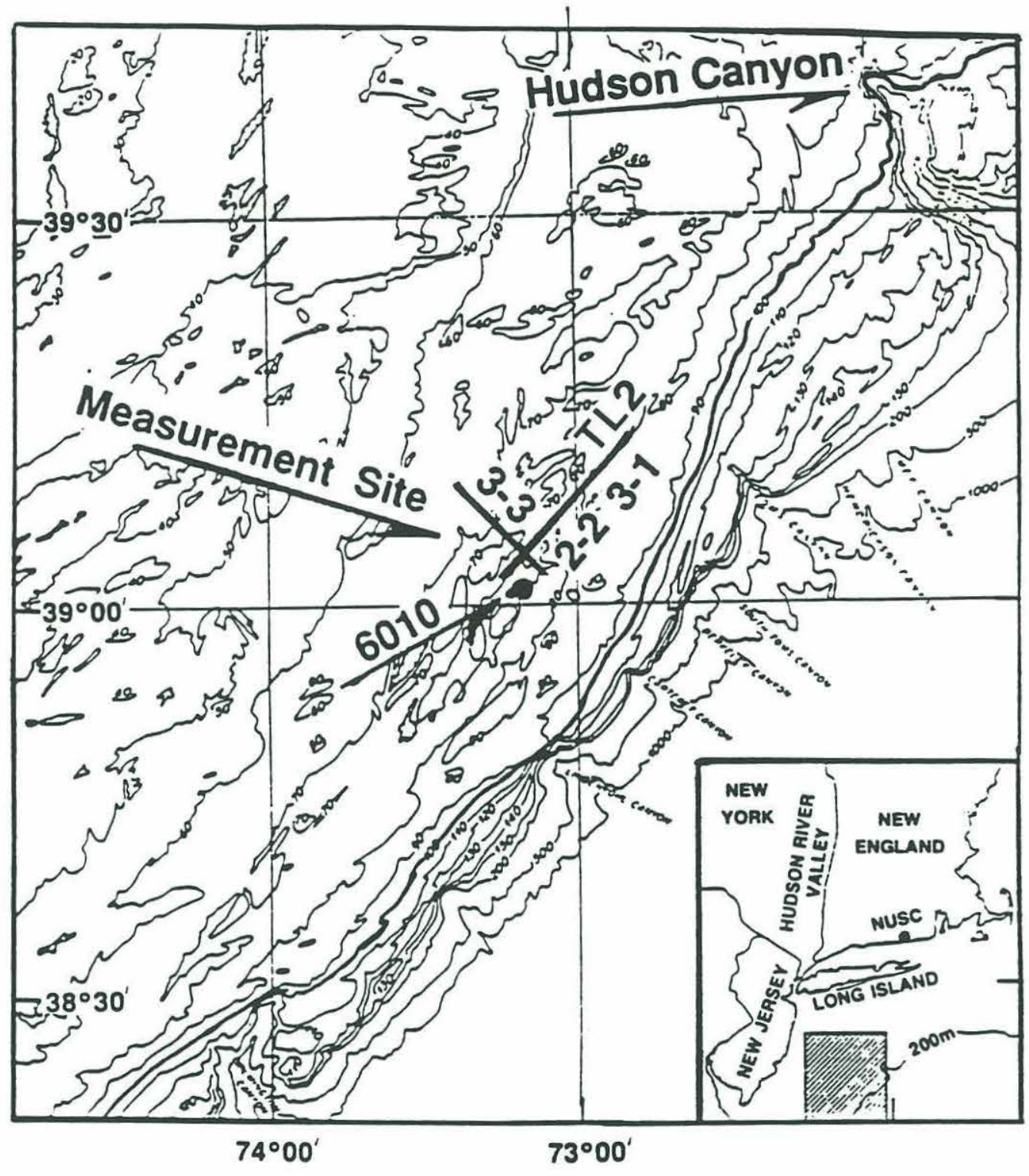

Figure 6-8: Hudson Canyon experimental area [86]. 


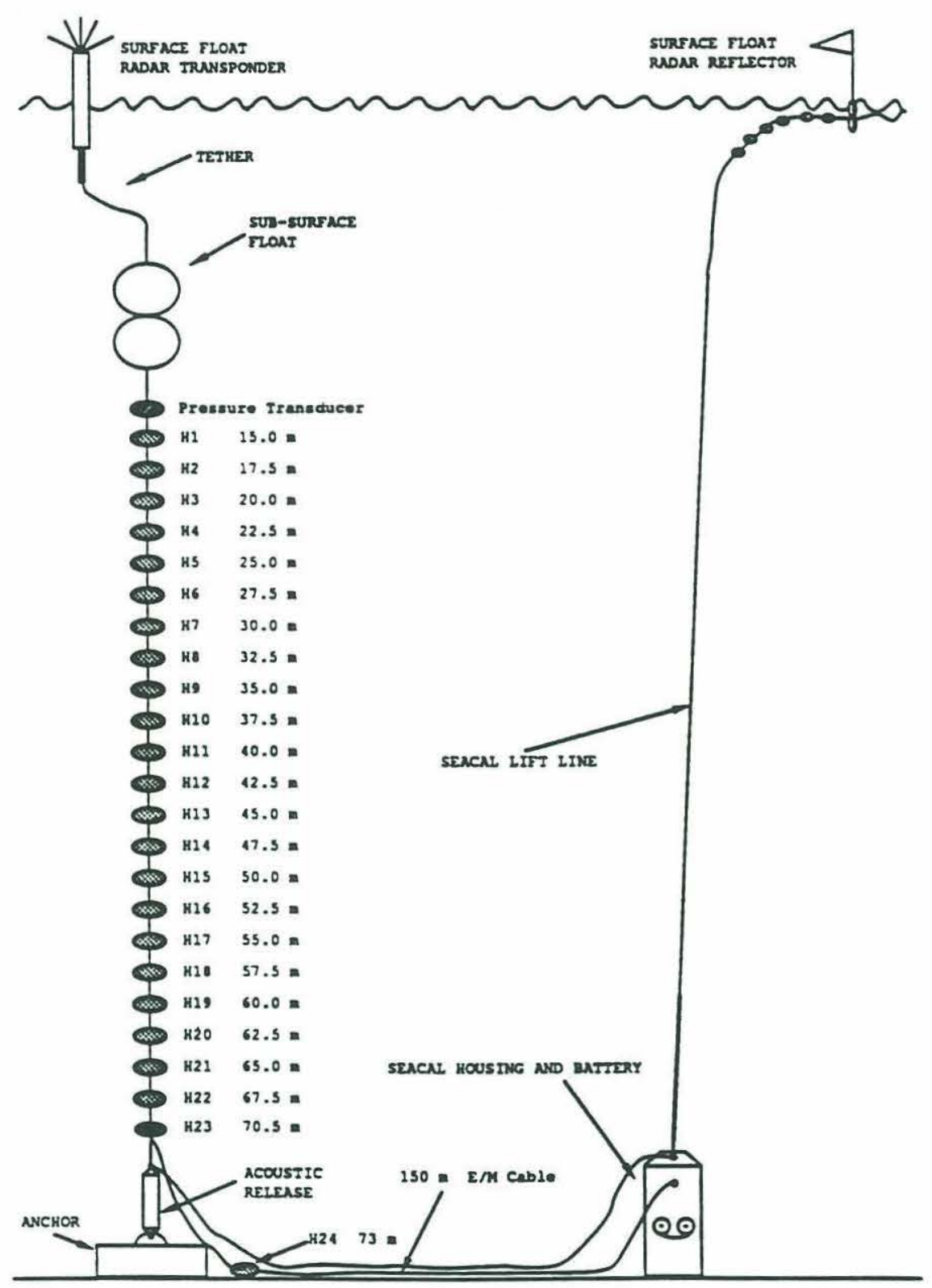

Figure 6-9: Vertical array of receivers $[85,86]$. 


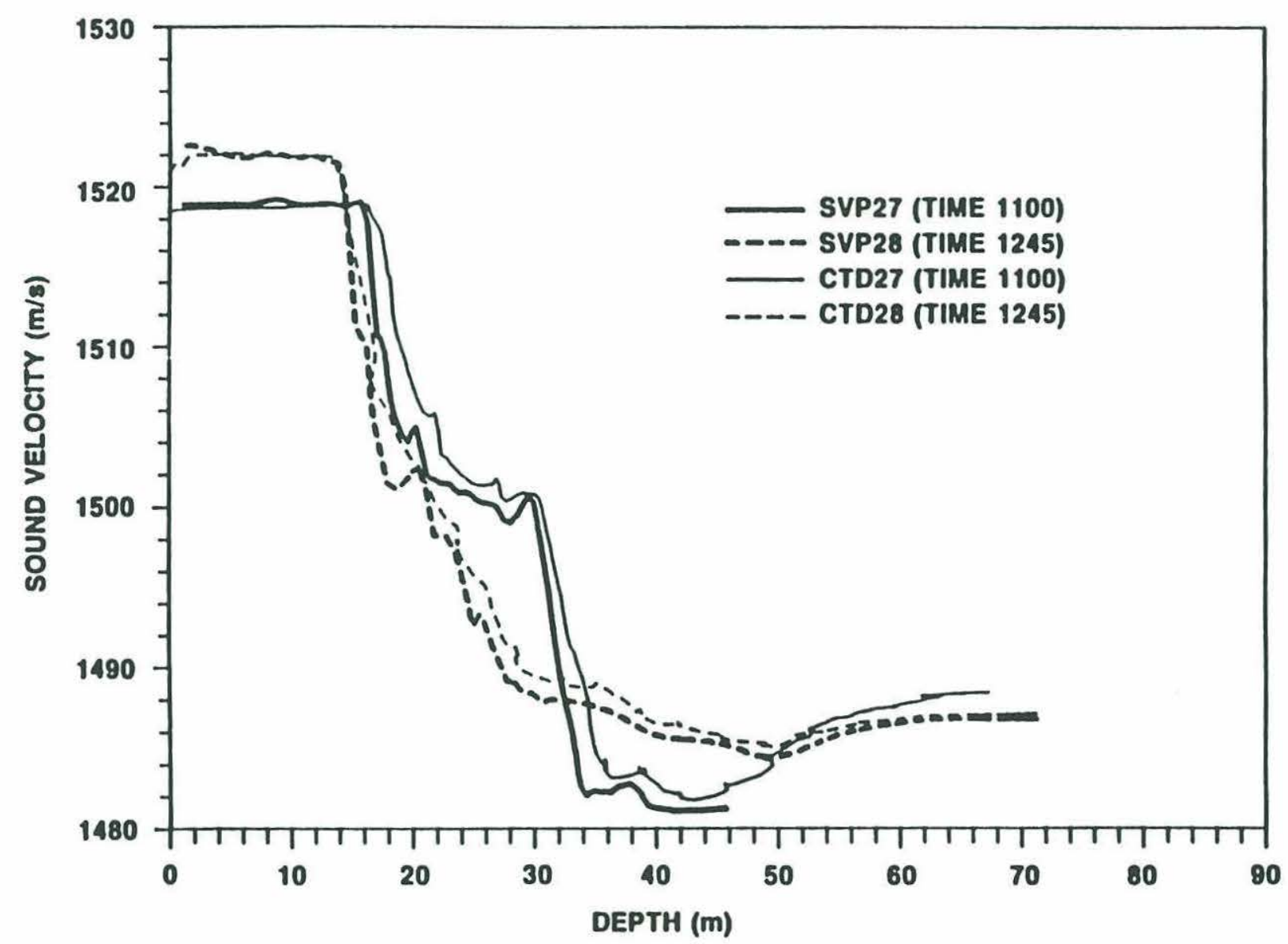

Figure 6-10: Sound speed measured along the track TL2 [86]. 


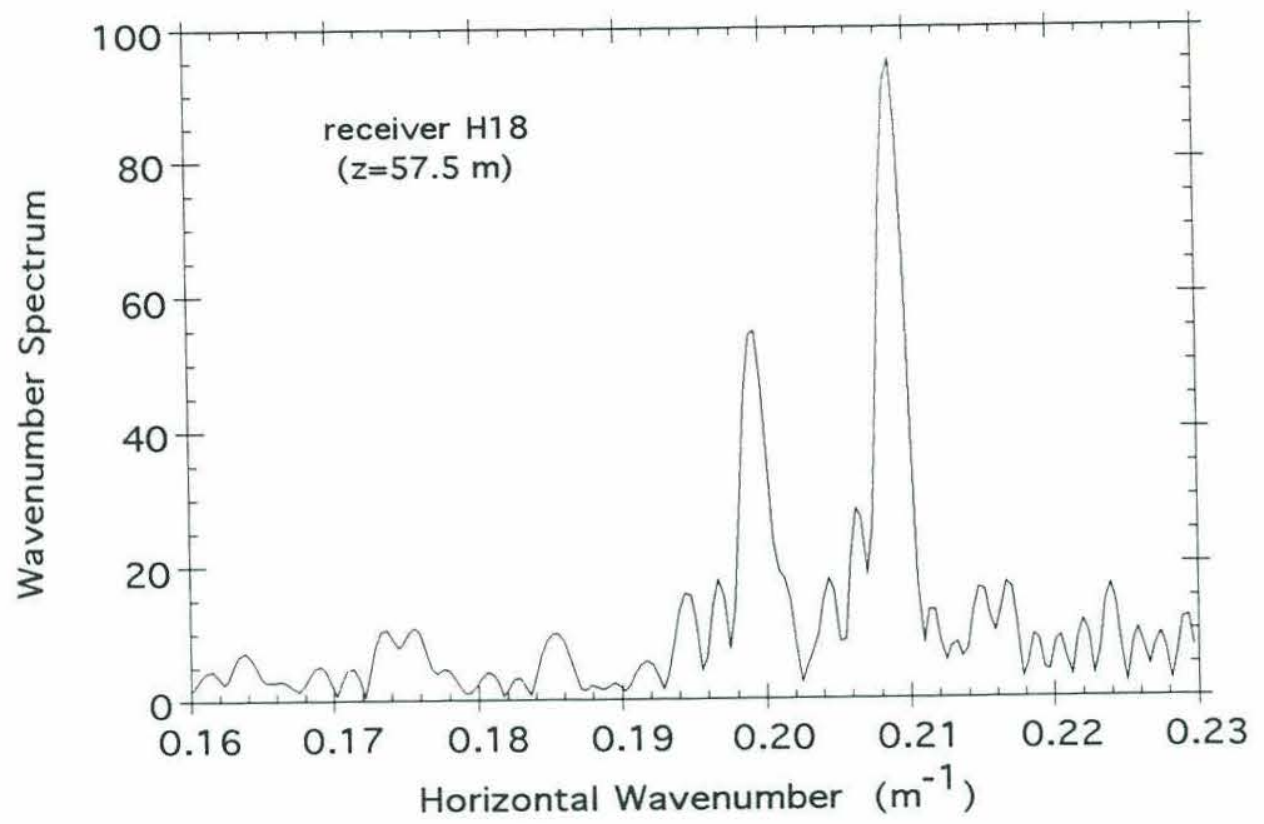

(a)

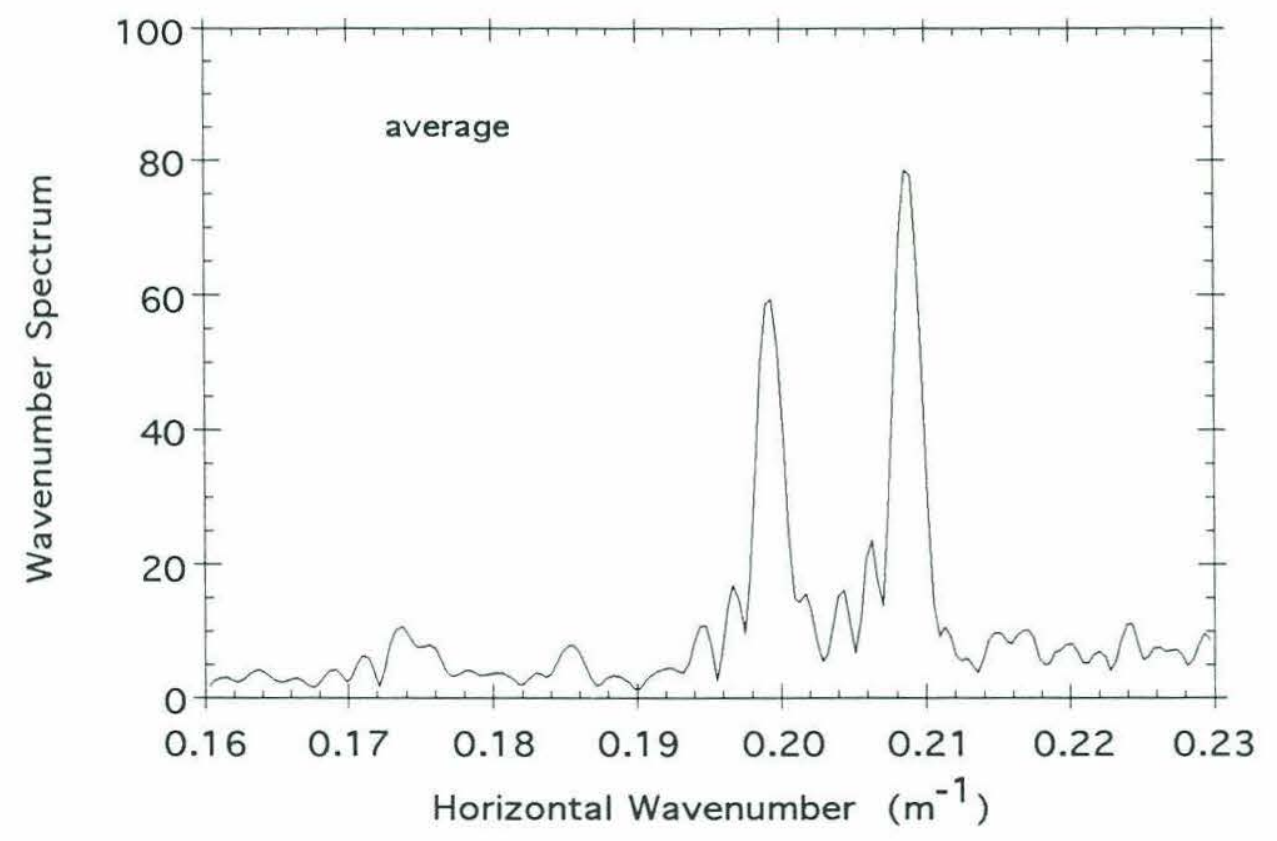

(b)

Figure 6-11: Wavenumber spectrum obtained by applying the asymptotic Hankel transform to the pressure field over the entire $4000 \mathrm{~m}$ range. (a) Receiver H18 and (b) average for all receivers. 


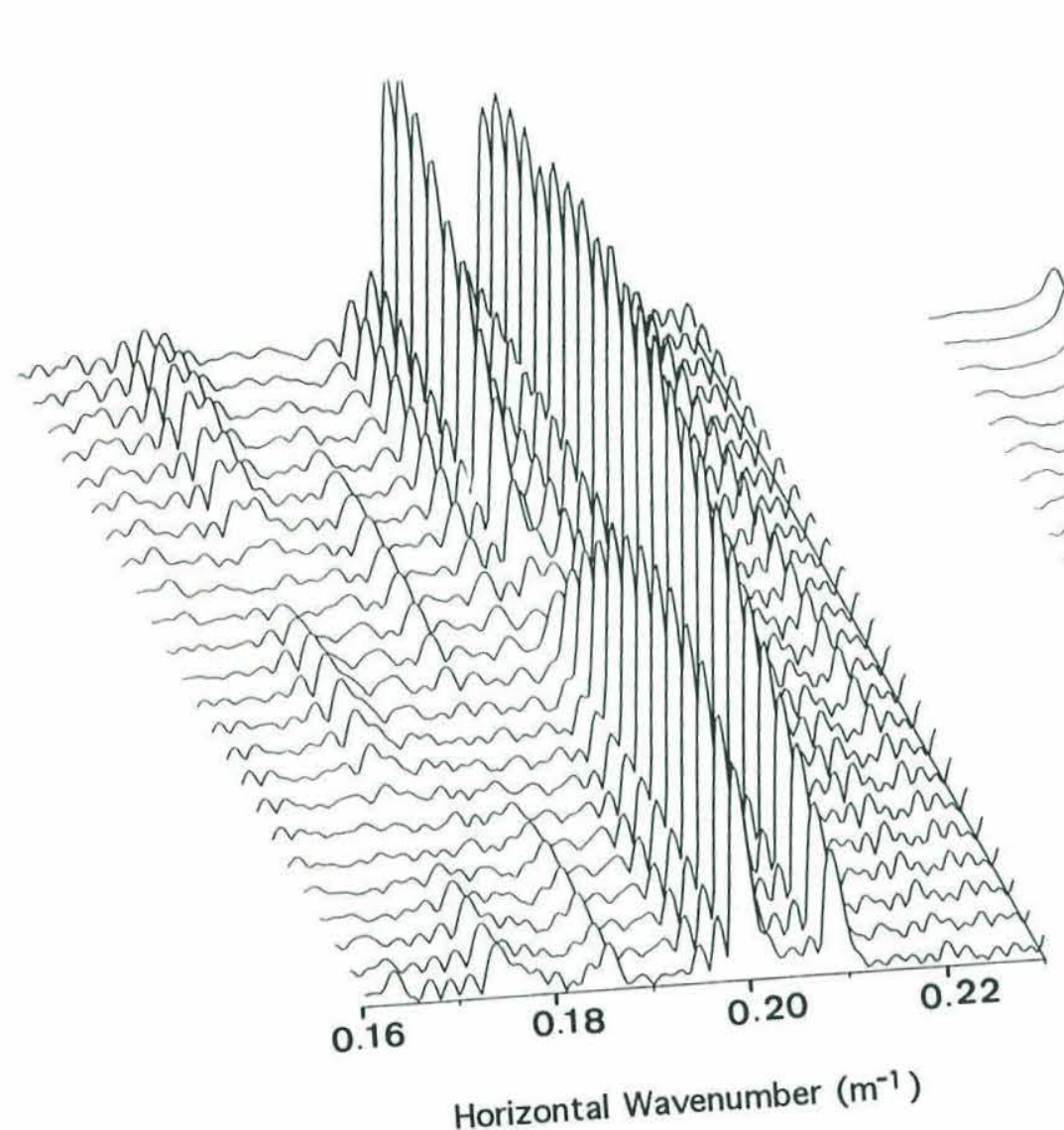

(a)

\section{)}

(b)

Figure 6-12. Wavenumber spectra at different receivers. (a) Measured and (b) simulated data. 


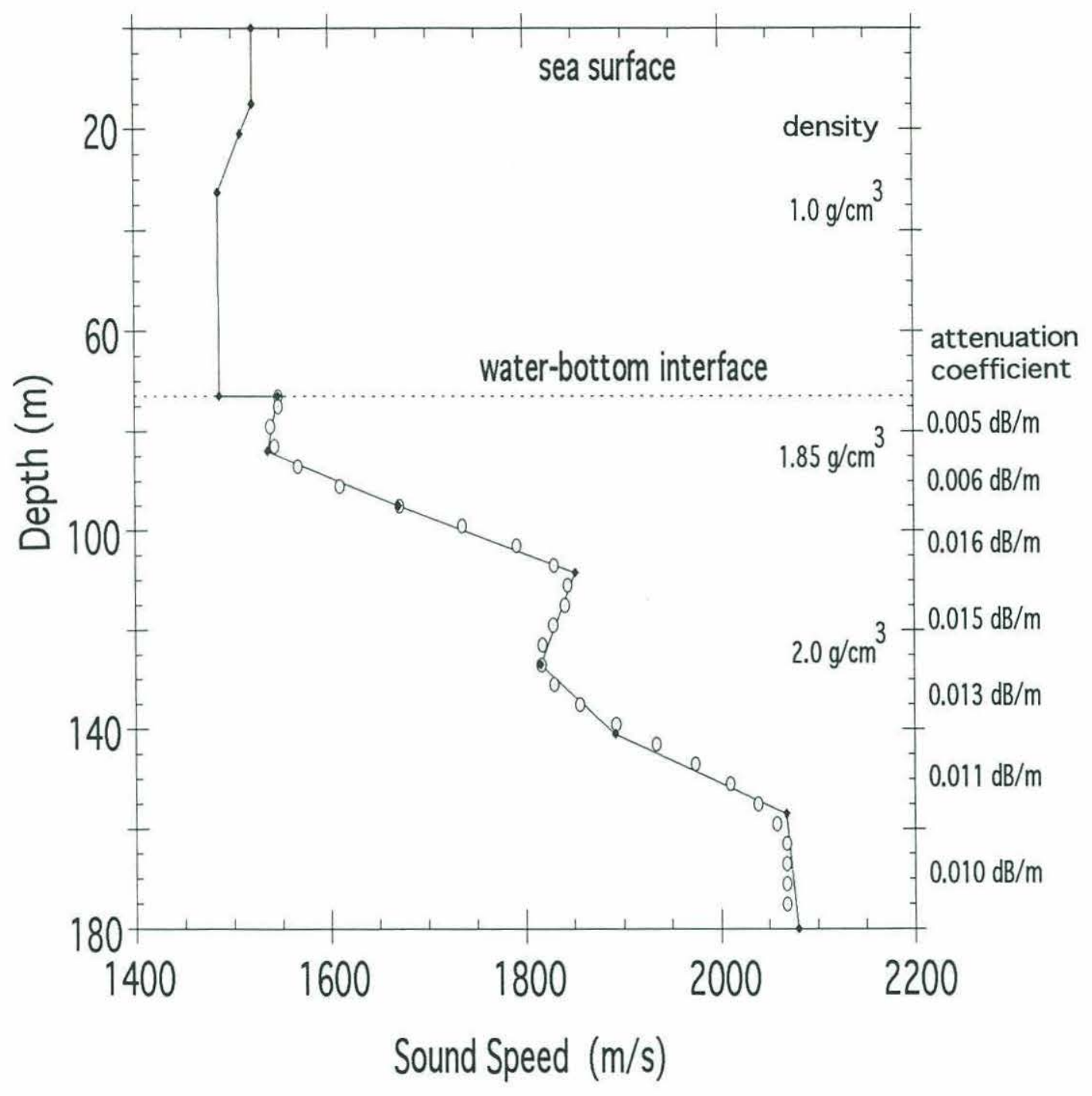

Figure 6-13: Sound speed $(\odot)$ obtained by a perturbative inversion method [Rajan, 50] and the $n^{2}$-linear fit $(\bullet)$. 
By using this $n^{2}$-linear profile in the depth equation (Eq.(2-3)), we can calculate the normal modes. The resulting eigenvalues are listed in Table 6-1 and are compared with the peak positions of the spectrum. It can be confirmed that both values agree well. Figure 6-14 shows a set of eigenfunctions for these modes. By substituting these eigenvalues and eigenfunctions into Eq.(2-6), we simulated the pressure field and compared it with the measured one in Figure 6-15. Again it can be confirmed that both pressure fields agree very well. We can also observe in Figures 6-15(a) and (c) a bimodal interference pattern in the output of receivers $\mathrm{H8}(\mathrm{z}=32.5 \mathrm{~m})$ and $\mathrm{H} 18(\mathrm{z}=57.5 \mathrm{~m})$, respectively. This arises because the pressure at these depths is dominated by the first and second modes, which can be seen from the two strong peaks in Figure 6-12(a). The interference distance, or so-called "skip distance", is given by $2 \pi / \Delta \kappa$, where $\Delta \kappa$ is the difference between eigenvalues of adjacent modes. From Table 6-1, we have $\Delta \kappa \equiv \kappa_{1}-\kappa_{2} \approx 0.0094$ and thus the skip distance becomes $668 \mathrm{~m}$, which agrees well with the interference distance shown in Figures 6-15(a) and (c). In contrast, we do not observe a regular interference pattern in Figure 6-15(b). This is due to the fact that the depth of receiver H14 ( $\mathrm{z}=47.5 \mathrm{~m})$ is close to the node of the second mode (see Figures 6-12(a) and 6-14) and this mode contributes less to the pressure.

We can obtain the wavenumber spectrum for the simulated pressure by using the same Hankel transform operation as that used for the measured pressure. Figure 6-12(b) represents a set of wavenumber spectra for the simulated field at the receiver depths shown in Figure 6-9. As discussed in Chapter 3.1, these spectra correspond to the depthdependent Green's function because of the horizontally stratified assumption. Both spectra agree very well with small differences in the behavior of the third and fourth modes only. This difference could be due to errors in the geoacoustic model including the assumption of range independence for the lower portion of the sediment column. 


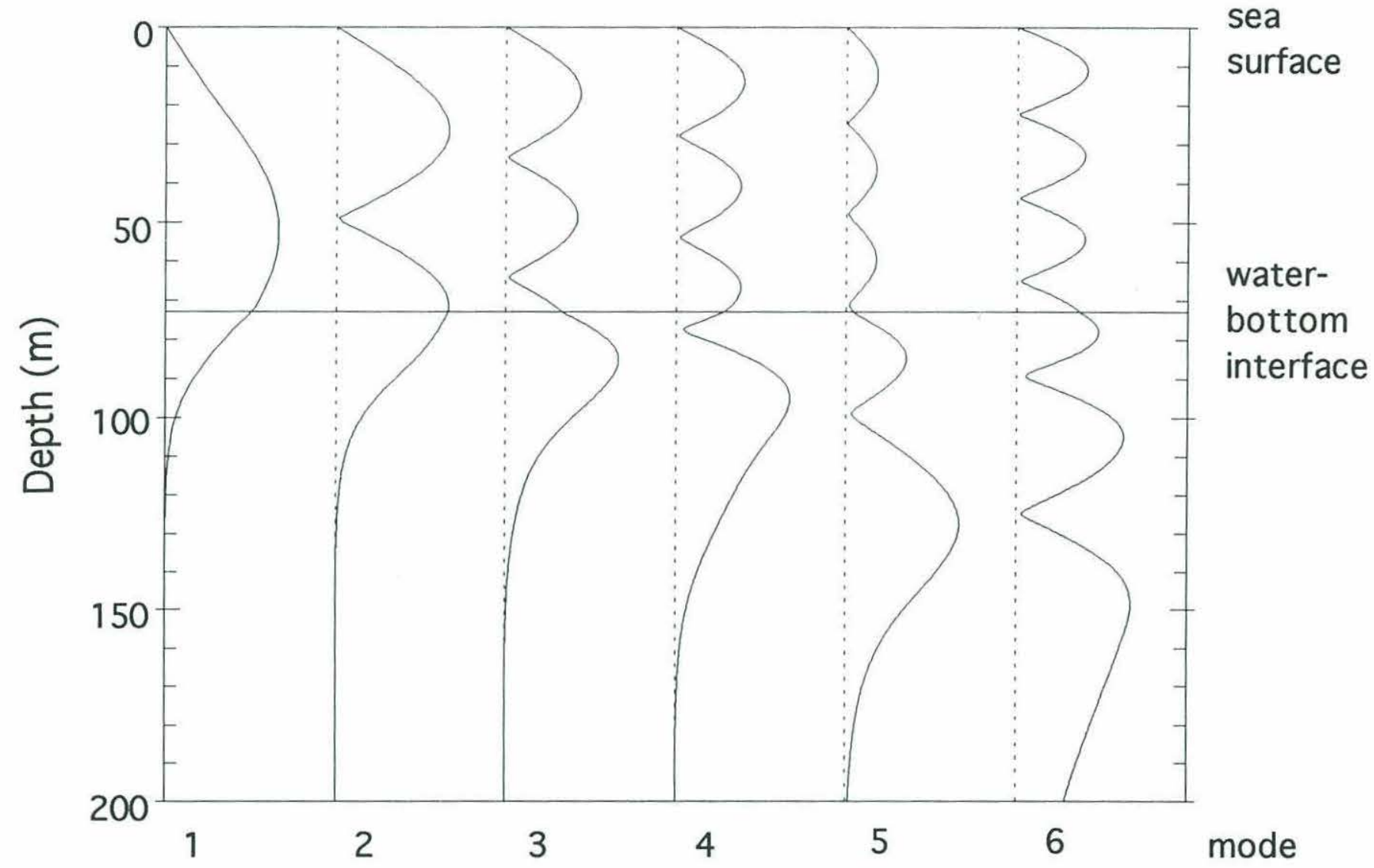

Figure 6-14: Normal mode eigenfunctions for the sound speed profile in Figure 6-13. 

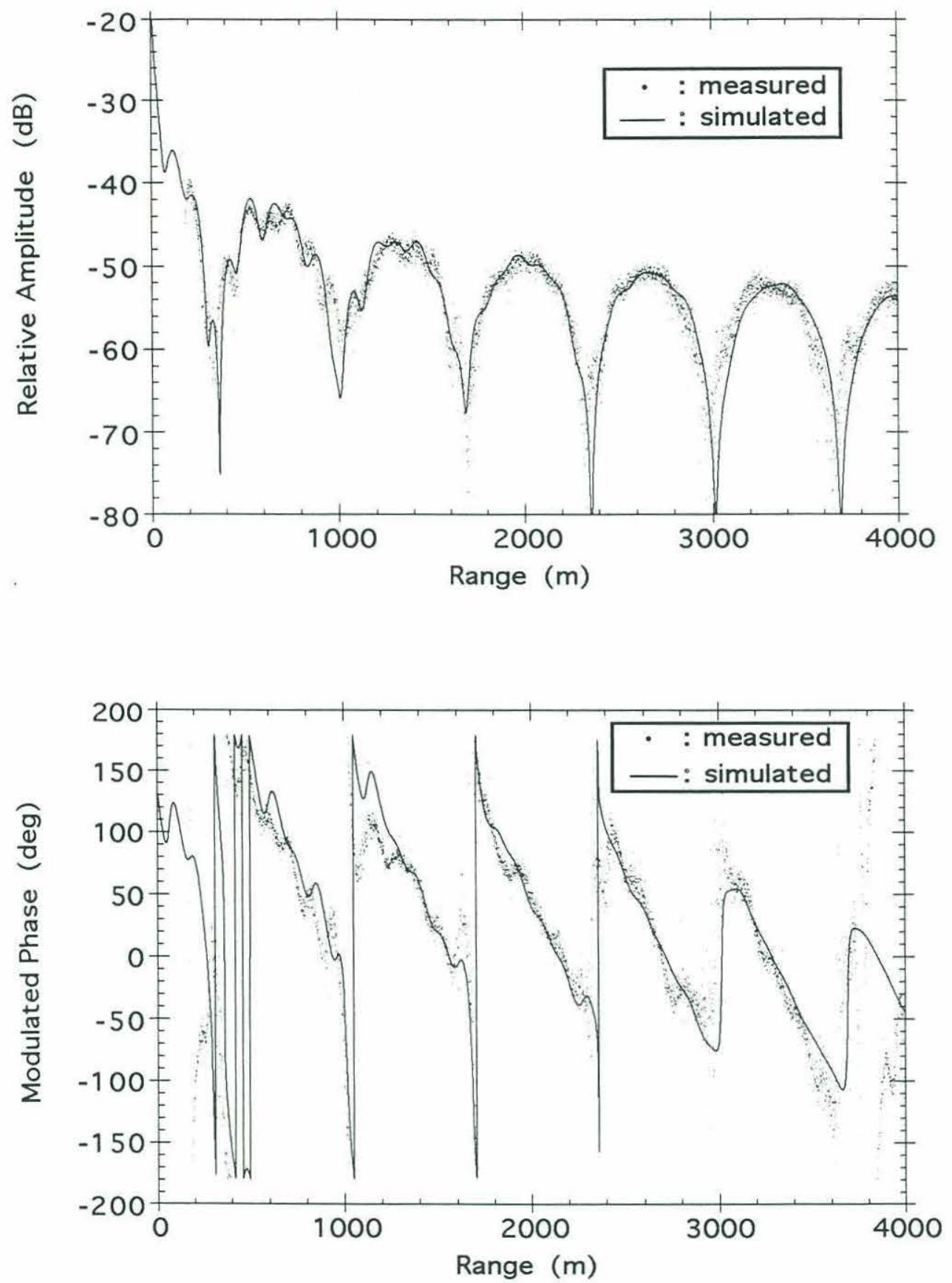

(a)

Figure 6-15: Comparison of the measured and simulated acoustic pressure fields. Phases are modulated as $\Phi(r) \rightarrow \Phi(r)-k_{0} r$ with $k_{0}=\omega / c_{0}$ and $c_{0}=1500 \mathrm{~m} / \mathrm{s}$. Receivers (a) H8, (b) H14, and (c) H18. 

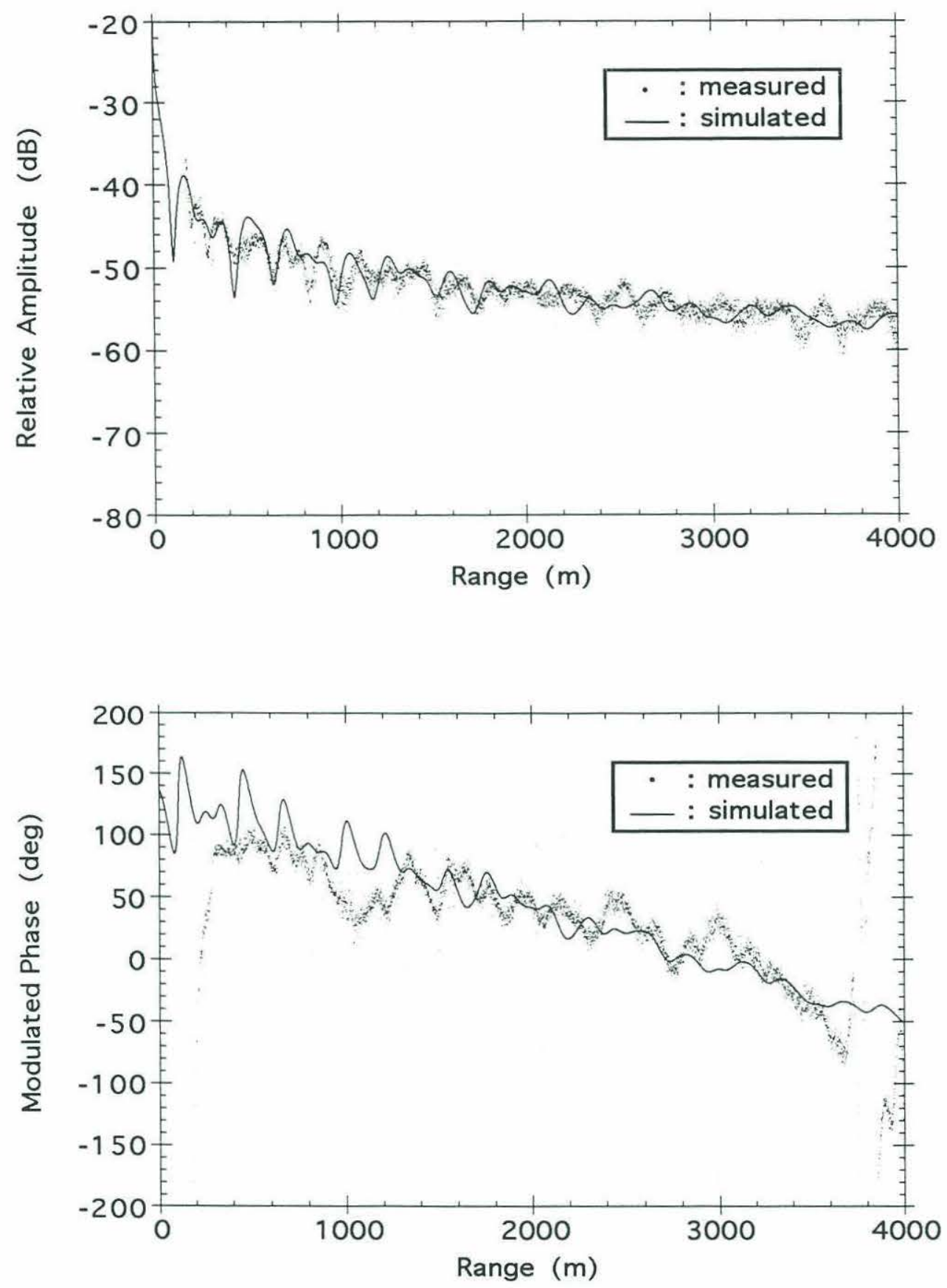

(b)

Figure 6-15, continued. 

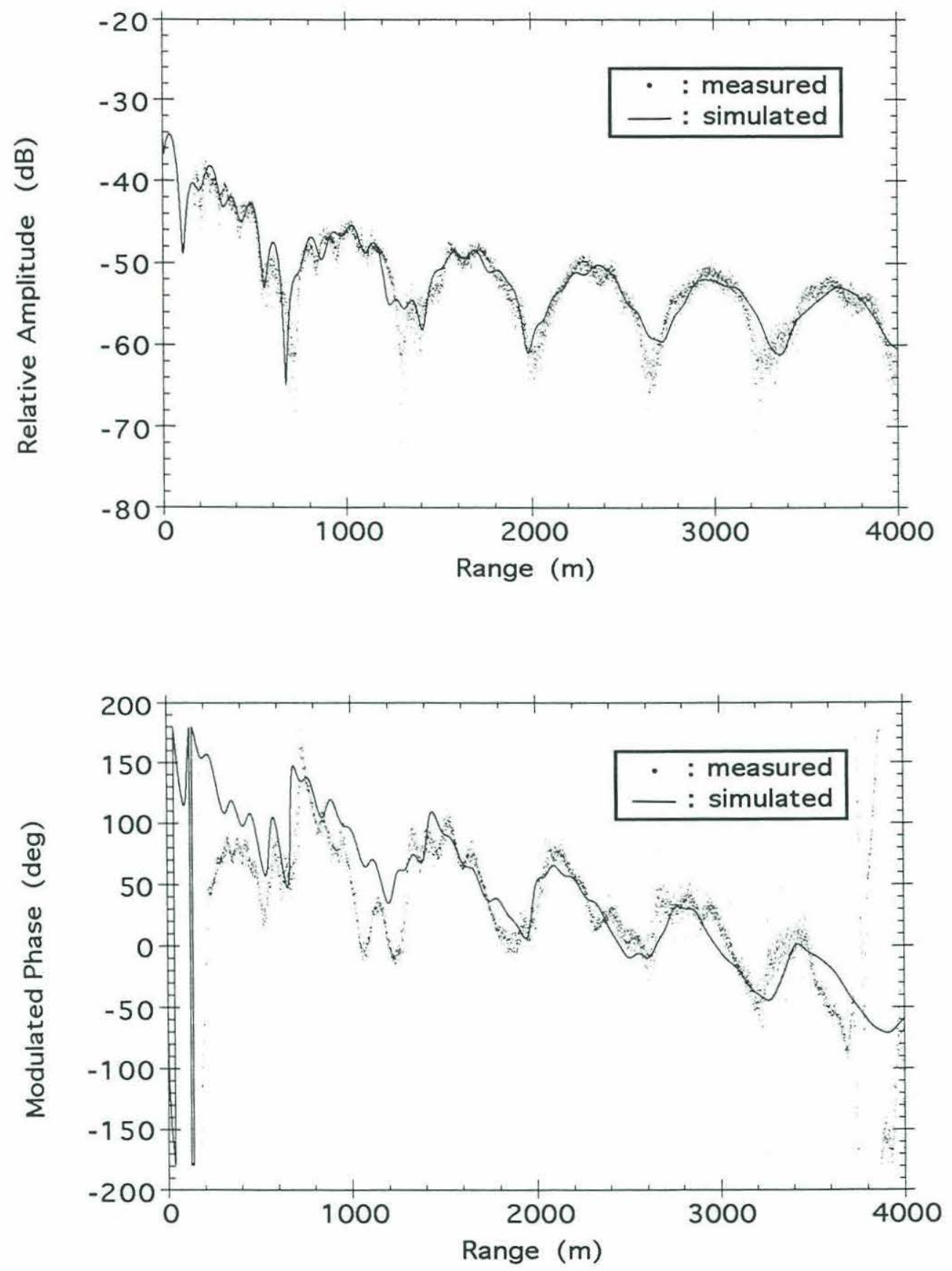

(c)

Figure 6-15, continued. 
Next, in order to examine the range dependence of the eigenvalues, let us first try a sliding window in the asymptotic Hankel transform. Since the field measurements in this experiment were executed in two directions, i.e., parallel and perpendicular to the slope (Figure 6-8), we cannot utilize the general Hankel transform due to a lack of field data for varying azimuths. Thus, on the assumption of weak refraction in the horizontal direction, we will use a zero-order asymptotic Hankel transform with a sliding window. Figure 616 shows the output of this transform when using the Hanning window with a length of $1000 \mathrm{~m}$. As seen from the interference pattern, the first and second modes interfere with each other in the output spectrum. Also we cannot recognize the spectral peaks corresponding to the third and fourth modes. Thus we cannot determine the local eigenvalues from this resulting spectrum.

Hence, let us separate the pressure by individual modes with the use of mode filtering. By assuming that the sound speed profile at the array site is the same as that in Figure 6-13, we may use the mode eigenfunctions shown in Figure 6-14 in Eq.(3-58). Here we set $N=6$ in this equation because the sixth mode is the largest one whose phase velocity does not exceed the maximum sound speed $(2070 \mathrm{~m} / \mathrm{s})$ in the profile in Figure 613. To solve Eq.(3-58), we have to execute the general inverse shown in Eq.(3-64). In the present problem, however, Eq.(3-64) results in an unstable solution. This arises because one of the eigenvalues of the matrix in Eq.(3-67) takes on a small value as shown in Table 6-2. (Note that these eigenvalues in Table 6-2 must be distinguished from the modal eigenvalues in Table 6-1.) Therefore we can use the EDM with $\breve{I}=5$ in Eq.(3-76) to execute the generalized inverse. As a result, the output of mode filtering is shown in Figure 6-17, where it has been transformed into the spectrum by using the same Hankel transform as that used in Figure 6-16. As seen from a comparison with Figure 6-16, the first and second modes are separated well. The third mode, however, is not completely isolated. This could be due to an error in the eigenfunction for the third mode, which 
Table 6-2: Eigenvalues of the matrix in Eq.(3-68).

$\begin{array}{cc}\dot{i}_{1} & \text { eigenvalue }\left(\boldsymbol{\lambda}_{\boldsymbol{i}_{1}}\right) \\ 1 & 0.6351 \\ 2 & 0.6041 \\ 3 & 0.5166 \\ 4 & 0.2864 \\ 5 & 0.0353 \\ 6 & 0.0012\end{array}$

is confirmed by a comparison of the spectra of the measured and simulated data: the depth of the second null in the measurement is closer to the water-bottom interface than that in the simulation (see Figure 6-12). To improve this result, we need to know more exactly the sound speed at the array site.

In order to examine modal evolution further, the peak trajectories in Figure 6-17 are plotted in Figure 6-18. The trajectories of the first and second modes are relatively stable as was previously expected from the spectra in Figures 6-11 and 6-12. Since the turning point depth of the second mode is located at about $15 \mathrm{~m}$ below the water-bottom interface (see Figure 6-14), we can infer that the sediment in this region is nearly horizontally stratified. Although the local eigenvalue of the fourth mode is not as precise as those of the first and second modes as seen from Figure 6-17, we can see in Figure 6-18 that the trajectory fluctuates around a wavenumber of 0.1755 for ranges less than $2000 \mathrm{~m}$ whereas it fluctuates around a wavenumber of 0.1735 for ranges greater than $2000 \mathrm{~m}$; these wavenumbers correspond to the peak positions of the split peak of the fourth mode as observed in Figures 6-11 and 6-12.

As compared to the Nantucket Sound result, we find that the local eigenvalues along the track TL2 in the Hudson Canyon area are relatively stable with range and the sediment bottom in this direction can be inferred to be nearly horizontally stratified. 


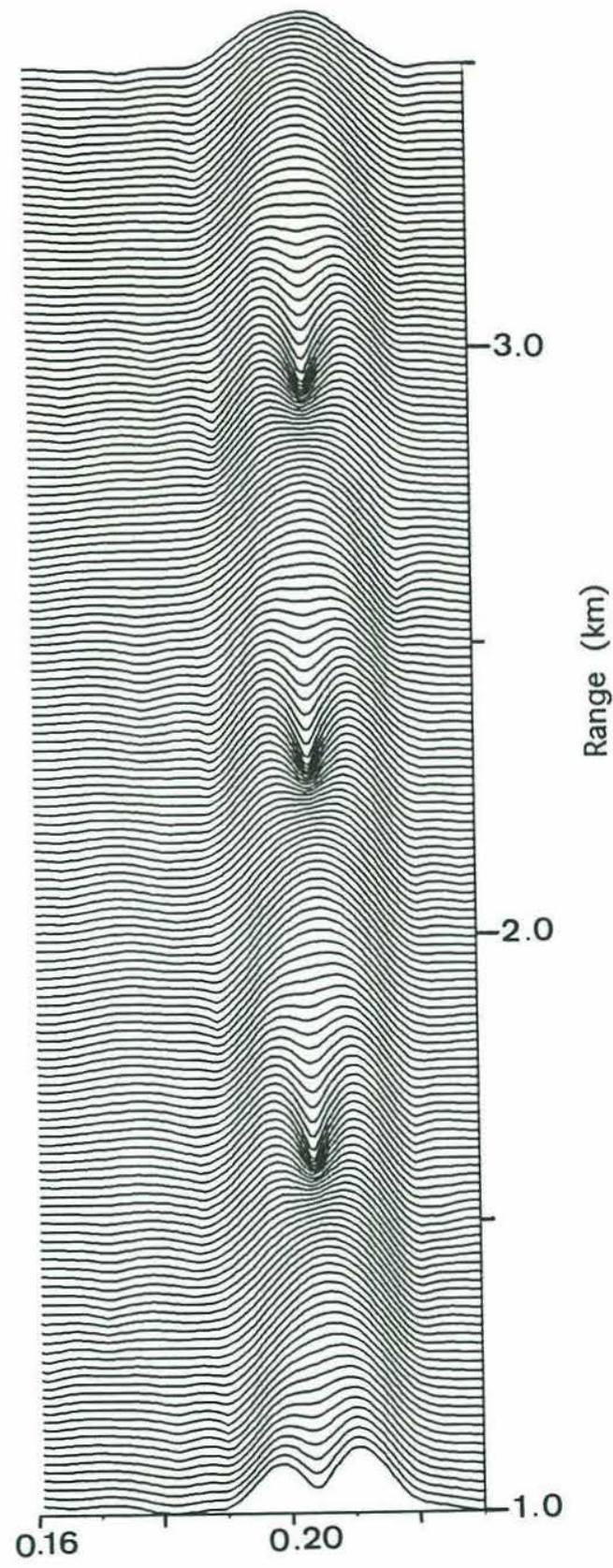

Horizontal Wavenumber $\left(\mathrm{m}^{-1}\right)$

Receiver $\mathrm{H8}$

$(z=32.5 \mathrm{~m})$
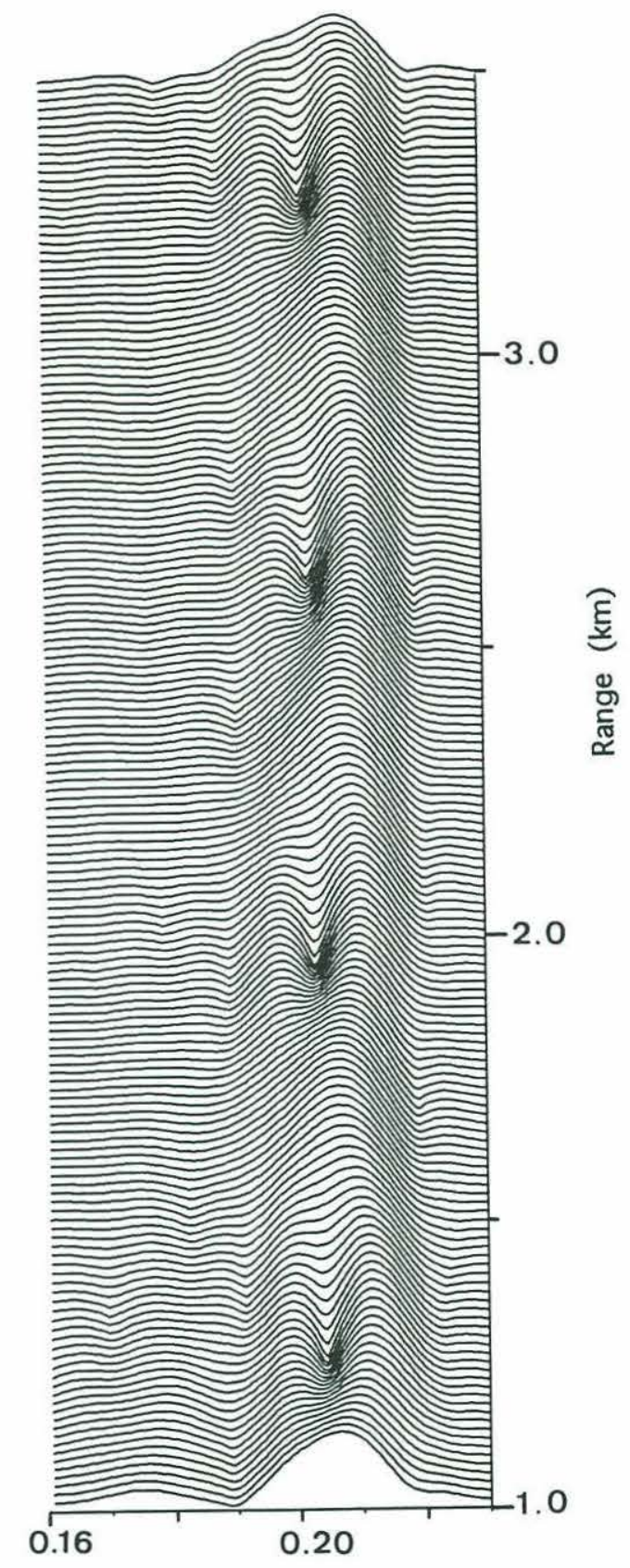

Horizontal Wavenumber $\left(\mathrm{m}^{-1}\right)$

$$
\begin{gathered}
\text { Receiver } \mathrm{H} 18 \\
(\mathrm{z}=57.5 \mathrm{~m})
\end{gathered}
$$

Figure 6-16: Range-dependent evolution of modal spectra obtained by applying the zero-order asymptotic Hankel transform with a sliding Hanning window of $1000 \mathrm{~m}$ length to the experimental data. 


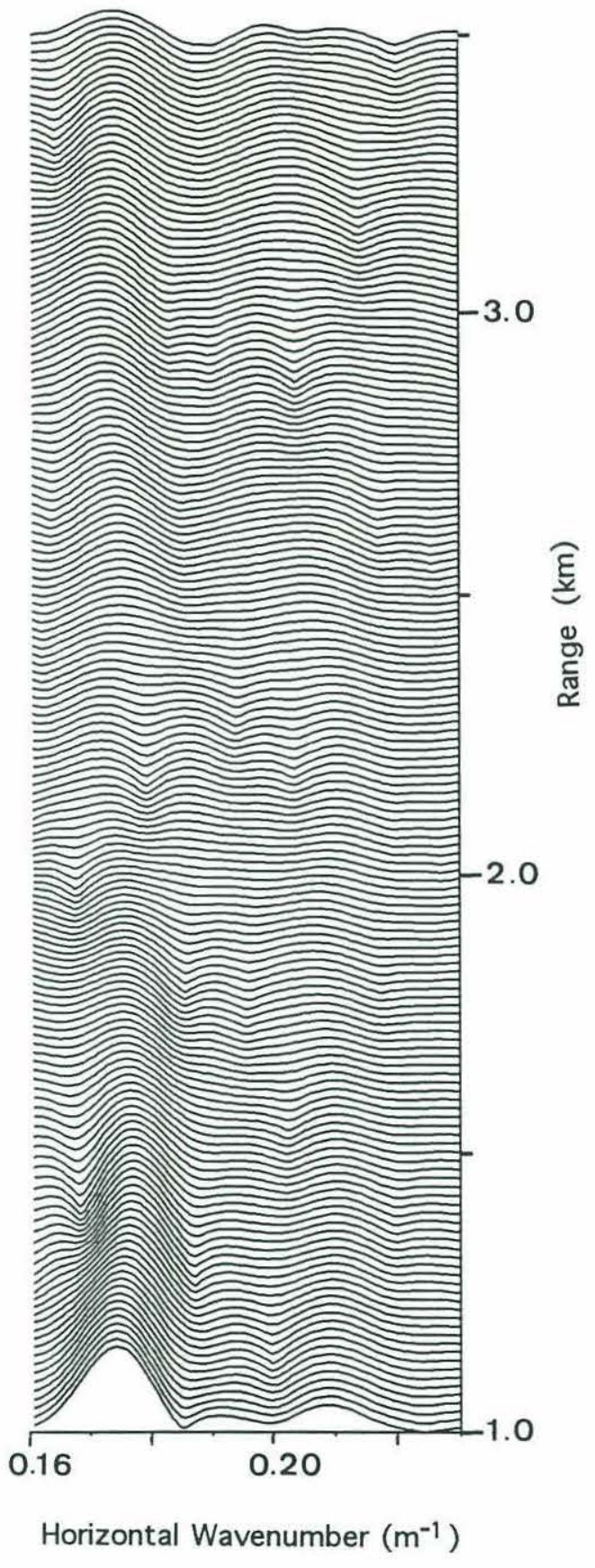

Mode 4

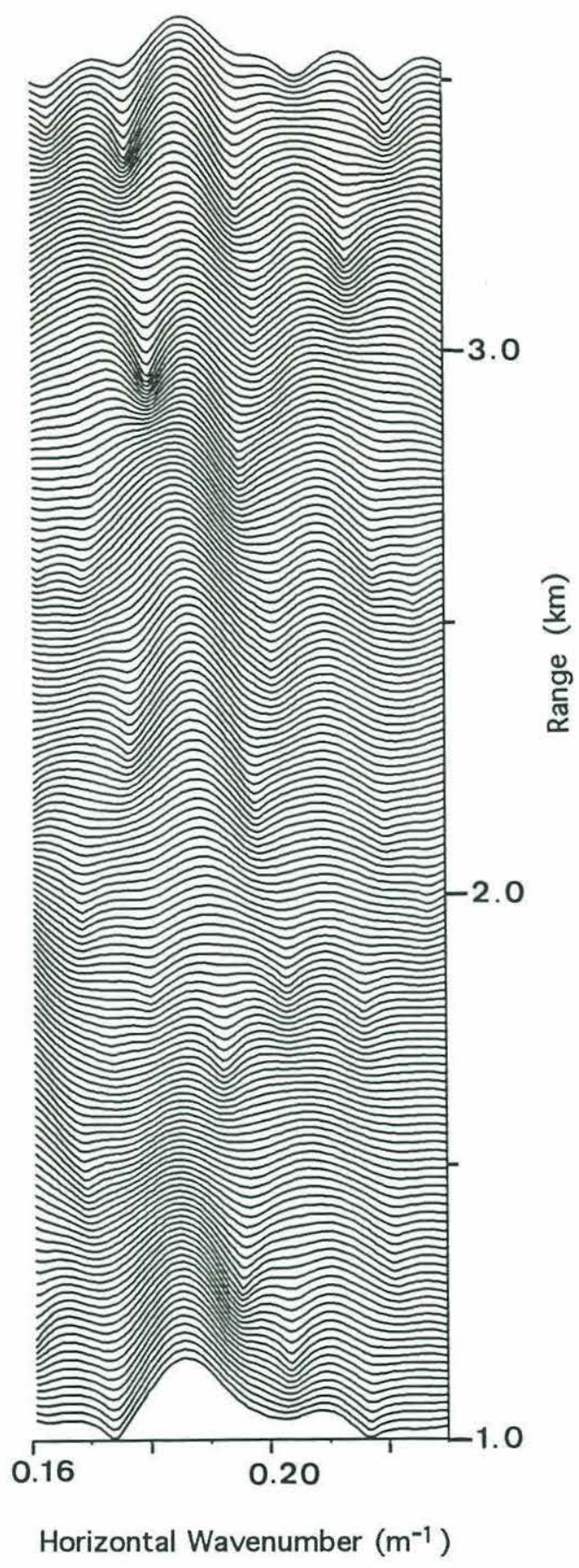

Mode 3

Figure 6-17: Range-dependent evolution of modal spectra obtained by applying the zero-order asymptotic Hankel transform with a sliding Hanning window of $1000 \mathrm{~m}$ length to the mode-filtered output. 


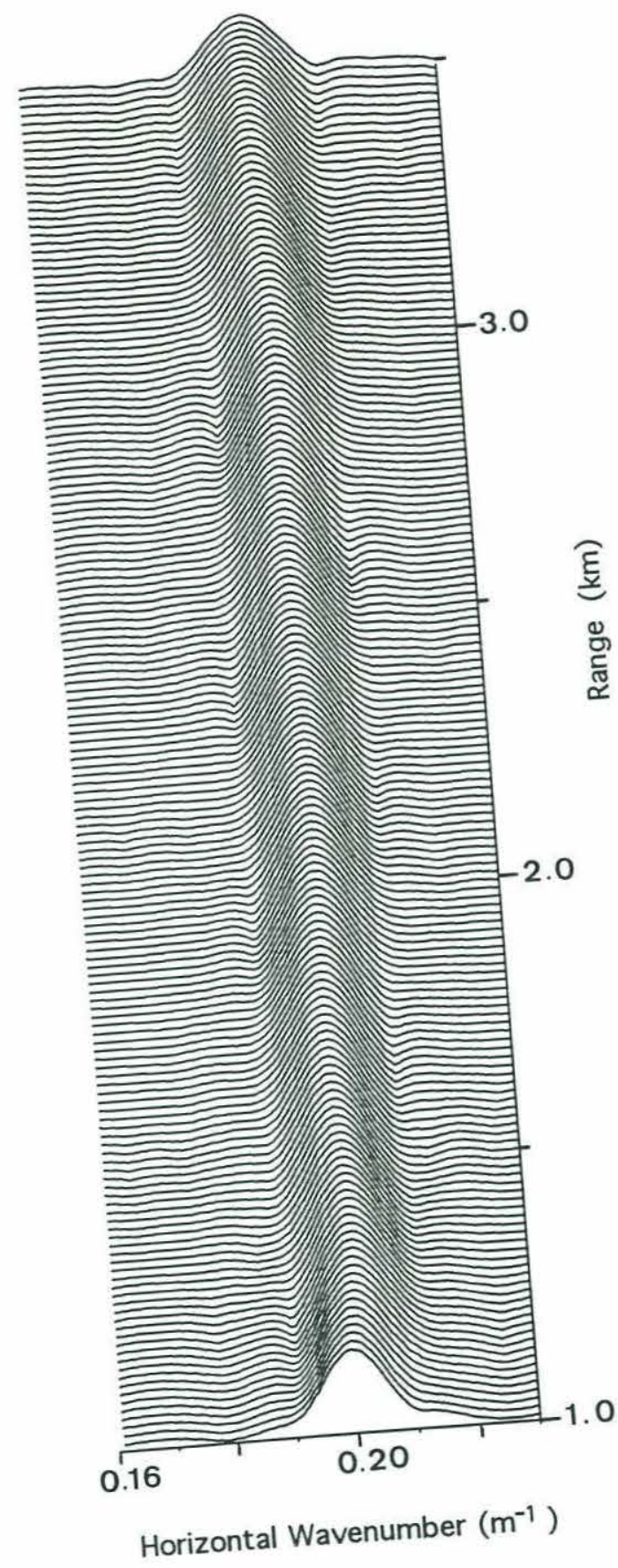

Mode 2

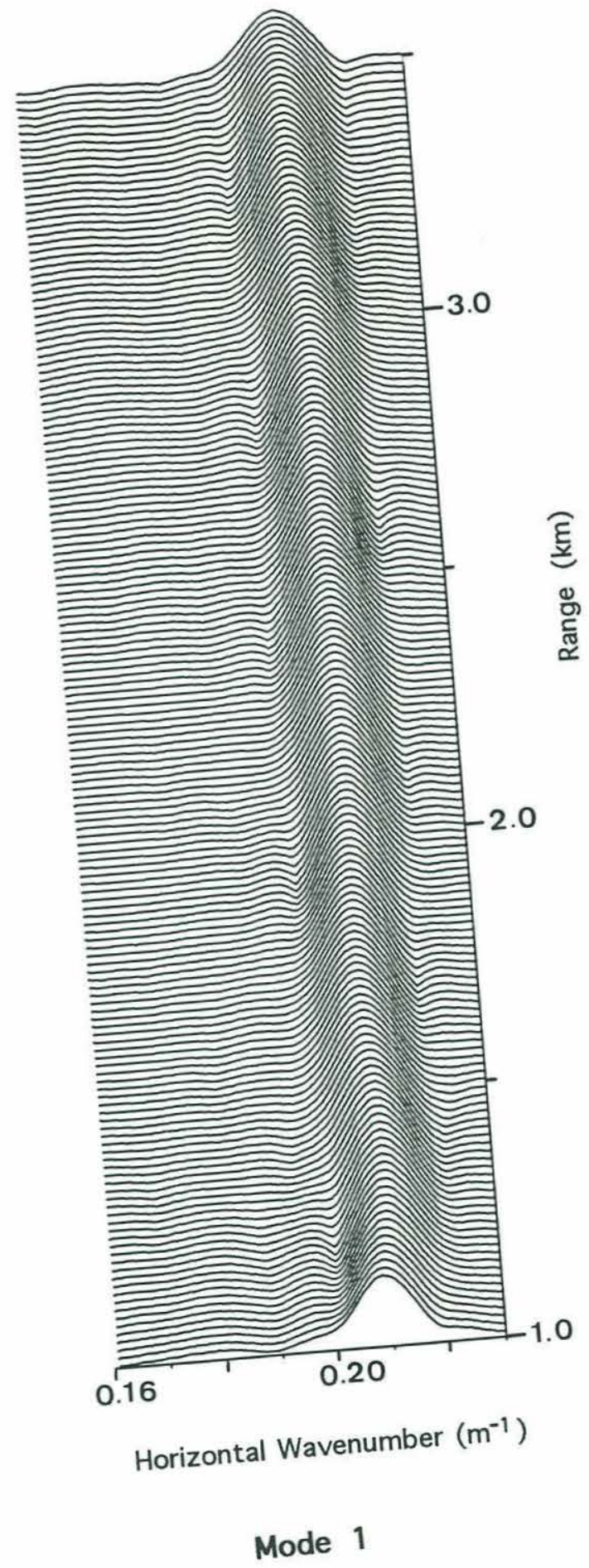

Mode 1

Figure 6-17, continued. 


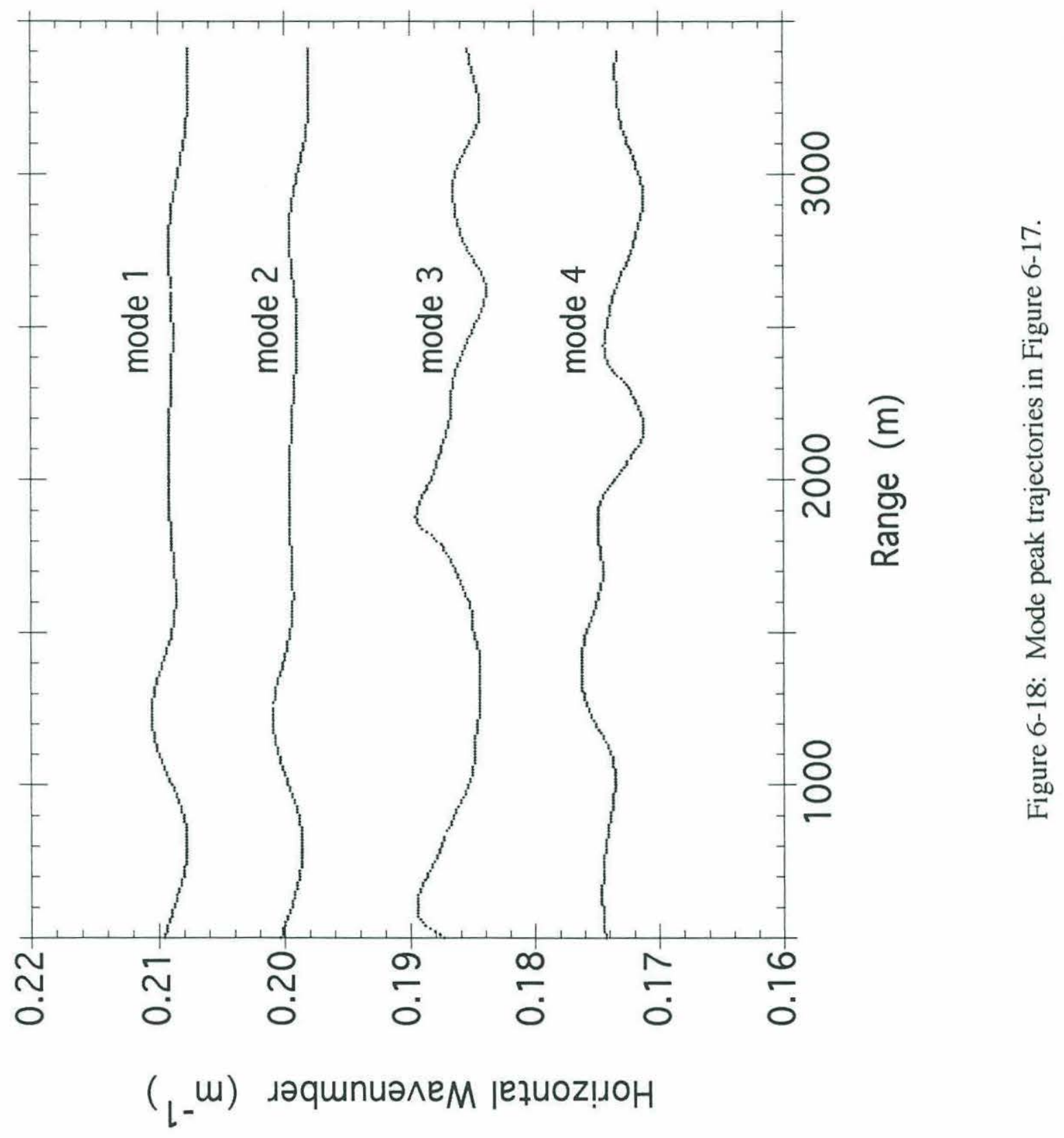




\section{Chapter 7}

\section{Conclusions}

In this final chapter we summarize the results obtained in Chapters 2 through 6 and briefly discuss some thoughts on future work.

In this thesis we focused on the analysis of the range evolution of local modes caused by horizontal variation of the sediment bottom in shallow water, the variation dealt with here is weak so as to allow us to utilize adiabatic mode theory. Since the local modes are subject to spatial changes of the sediment geoacoustic properties, we first tried to detect the local eigenvalues by using the asymptotic Hankel transform with a sliding window together with mode filtering. Next, in an attempt to obtain the spatial variation of the sediment properties from the range variation of the local eigenvalues, we derived an analytical relationship between these two variations by using a linear perturbation

method. Based on this relation, an inversion method for determining the range-dependent geoacoustic parameters in the sediment was developed.

In Chapter 2 we reviewed the normal mode and adiabatic mode theories for describing the acoustic pressure field. In order to cope with a 3-D varying waveguide, we also reviewed briefly the Nx2D method and the horizontal ray method.

In Chapter 3, in order to detect local eigenvalues in a range-dependent but cylindrically symmetric waveguide, we utilized the zero-order asymptotic Hankel transform with a short sliding window. The effect of the range-dependence of the local eigenvalues on the Hankel transform was examined analytically by expanding the phase term of the adiabatic mode field to fourth order. In a single mode situation, it was found that the departure of the peak position in the output spectrum from the local eigenvalue depends on both the second derivative of the local eigenvalue with respect to range and 
the window length and type. In addition to this error, another type of departure from the local eigenvalue is induced by the interference with the sidelobes of adjacent modes. In order to attain separation of the modes prior to the Hankel transform, we used mode filtering by incorporating data from a fixed vertical array of receivers. When this filtering process becomes unstable due to the involvement of a singular matrix, we can use the eigenvector decomposition method (EDM) and the stabilized least-mean-square method (SLMS) for reliable mode separation. At the end of Chapter 3, we applied the methods discussed in this chapter to the pressure field simulated numerically by using adiabatic mode theory in a model of a laterally inhomogeneous shallow water waveguide. The result indicated that the use of mode filtering improved the detection of the local eigenvalues. When the field included higher modes, it was demonstrated that the EDM was useful in providing a stable result for mode separation.

Chapter 4 was devoted to detection of the local eigenvalues in a 3-D varying shallow water environment. When we apply the zero-order asymptotic Hankel transform with a sliding window to the pressure field measured along a radial in a 3-D varying environment, then we underestimate the local eigenvalues due to the horizontal refraction effect. In order to deal with this problem, we explored the use of a general asymptotic Hankel transform with a 2-D sliding window in a cylindrical coordinate system. By expanding this transform with respect to the azimuthal angle, we demonstrated that the first term in the Taylor series corresponds to the zero-order Hankel transform used in the cylindrically symmetric waveguide; the rest of the terms account for the difference between the underestimated and actual local eigenvalues. Next, we analyzed the effect of horizontal refraction by evaluating the general Hankel transform with the use of the stationary phase method. Based on this analysis, it was shown that we can determine the horizontal refraction angle and correct for the underestimated value of the local eigenvalues by using the refraction angle. 
In order to re-examine analytically the effect of horizontal refraction, we also derived an alternative representation for the 2-D Fourier transform by using the phase difference between the outputs of the mode filter, which was applied to the pressure field in the different azimuthal directions. By comparing the resulting transform with the zero-order asymptotic Hankel transform, we demonstrated that the underestimated amount for the local eigenvalues in the latter transform can be determined by using the phase difference in mode filtering.

At the end of Chapter 4 we examined numerically the horizontal refraction effect by applying the method discussed in that chapter to the pressure field simulated using the horizontal ray method. It was shown that we can determine the horizontal refraction angle by using the general Hankel transform, as long as the pressure field is sampled in the azimuthal direction so as to satisfy the spatial Nyquist criterion. This issue was also discussed in connection with the design of experiments for measuring pressure fields in a 3-D varying, shallow water environment.

In Chapter 5, we discussed a method for determining the range-dependent properties of the sediment bottom from the local eigenvalues. First, by utilizing a linear perturbation method, we derived the analytical relationship between the range variation of the local eigenvalues and the spatial change in the bottom properties in a horizontally and vertically varying, multilayered bottom model. This relation was checked analytically by using the range-dependent Pekeris waveguide, for which the identical relation can be obtained by differentiating the characteristic equation. Next, by representing the sound speed in each layer by an $n^{2}$-linear curve, we showed that the integral in the perturbation relation can be executed in closed form. As a result, we saw that the range variation of the local eigenvalues can be separated into terms that depend on the range variations of the sediment interface depth, the sound speed and gradient in each sediment layer, and the sound speed profile in the water column. Finally, based on this perturbation relation 
between the local eigenvalues and the geoacoustic parameters, we demonstrated that we can invert for the geoacoustic parameters at successive ranges.

In order to check numerically the methods derived in this chapter, we applied them to the shallow water model used in Chapter 4. As a result, we showed that we can accurately estimate the unknown range-dependent geoacoustic parameters in this model as long as the local eigenvalues are precisely provided. When the number of local modes is less than the number of unknown geoacoustic parameters, we can employ the singular value decomposition (SVD) method and observe that the proper choice of modes can reduce the error in the estimation of the parameters.

In Chapter 6 we analyzed some experimental data by utilizing the asymptotic Hankel transform with a sliding window and mode filtering. In the Nantucket Sound experimental data, we found that the range variation of modes is dominated by the lateral variation of the geoacoustic parameters rather than by the bathymetric change. In the Hudson Canyon experimental data, we observed that the two lowest modes can be separated very well by mode filtering. We found that the range variation of modes along the track parallel to the shelf in the Hudson Canyon area is small compared to the Nantucket Sound result.

\subsection{Suggestions for future work}

In this thesis, we examined the effect of range variation of the sediment bottom on the local eigenvalues in a framework for which adiabatic mode theory holds. If, however, the sediment properties have strong variations in the horizontal direction, then we have to take into account the effect of mode coupling. To do this, we would first have to simulate pressure fields which accommodate mode coupling in the propagation process. An analytical study of the Hankel transform of this field would also be required in order to understand the output of this transform. 
In the inversion method discussed in this thesis, we assumed range independence for the attenuation coefficient in each sediment layer. In order to acquire the local character of the attenuation coefficient with respect to range, we would have to measure the amplitude of the local spectrum. Also we would need to develop the theoretical relationship between the range variations of the spectral amplitude and the attenuation coefficient.

The bottom model used here to relate the range variations of the local modes and the sediment properties is based on the assumption of a fluid medium. In general, we need to include geoacoustic parameters associated with shear waves as well. Also, attention has to be paid to the effect of conversion between the shear and compressional waves due to the range variation.

If we use a broadband signal as the source, then we can make use of group velocity dispersion curves as tools for estimating the geoacoustic properties. In a range-dependent shallow water environment, the modal group velocity becomes a function of range and could be measured for individual modes by applying mode filtering to data obtained on a fixed vertical array of receivers from a source whose position is changing with range.

By taking into account the effects stated above, we could deal with more general problems associated with the horizontal and vertical variation of the sediment bottom. 


\section{Appendix A}

In this appendix, we prove Eq.(5-15), which was used when deriving the perturbation of the local eigenvalues in Chapter 5.2.

Let us consider Eq.(5-5), evaluated at slightly separated positions on the sediment interface, i.e., $(r, h(r))$ and $(r+\Delta r, h(r+\Delta r))$ :

$$
\left.u_{n}(r, z)\right|_{\mid=h_{j}(r)-0}=\left.u_{n}(r, z)\right|_{z=h_{j}(r)+0}
$$

and

$$
\left.u_{n}(r+\Delta r, z)\right|_{\mid=h_{j}(n+\Delta r)-0}=\left.u_{n}(r+\Delta r, z)\right|_{z=h_{j}(n+\Delta r)+0} .
$$

Subtracting Eq.(A-1) from Eq.(A-2), dividing both sides by $\Delta l\left(\equiv\left\{(\Delta r)^{2}+[h(r+\Delta r)\right.\right.$

$\left.\left.-h(r)]^{2}\right\}^{\frac{1}{2}}\right)$, and taking the limit as $\Delta l \rightarrow 0$ yields

$$
\begin{aligned}
& \lim _{\Delta l \rightarrow 0} \frac{\left.u_{n}(r+\Delta r, z)\right|_{\left|=h_{j}(r+\Delta r)-0-u_{n}(r, z)\right|_{=h_{j}(r)-0}}}{\Delta l}, \\
& =\lim _{\Delta l \rightarrow 0} \frac{\left.u_{n}(r+\Delta r, z)\right|_{z=h_{j}(r+\Delta r)+0-\left.u_{n}(r, z)\right|_{=h_{j}(r)+0}}}{\Delta l},
\end{aligned}
$$

Eq.(A-3) is thus expressed using the derivative tangential to the sediment interface, $\frac{\partial}{\partial l}$, as

$$
\left.\frac{\partial u_{n}}{\partial l}\right|_{z=h_{j}(r)-0}=\left.\frac{\partial u_{n}}{\partial l}\right|_{z=h_{j}(r)+0} .
$$


Here $\frac{\partial}{\partial l}$ can be replaced with $\frac{\partial}{\partial l}=\frac{1}{\sqrt{1+h_{j}^{\prime 2}(r)}}\left(\frac{\partial}{\partial r}+h_{j}^{\prime}(r) \frac{\partial}{\partial z}\right)$, so that Eq.(A-4) becomes

$$
\left[\frac{\partial u_{n}}{\partial r}+h_{j}^{\prime}(r) \frac{\partial u_{n}}{\partial z}\right]_{z=h_{j}(r)-0}=\left[\frac{\partial u_{n}}{\partial r}+h_{j}^{\prime}(r) \frac{\partial u_{n}}{\partial z}\right]_{z=h_{j}(r)+0},
$$

where $\sqrt{1+h_{j}^{\prime 2}(r)}$ has been dropped because it takes on the same value on both sides.

Rearranging Eq.(A-5) leads to

$$
\left.\frac{\partial u_{n}}{\partial r}\right|_{z=h_{j}(r)-0}-\left.\frac{\partial u_{n}}{\partial r}\right|_{z=h_{j}(r)+0}=-\left(\left.\frac{\partial u_{n}}{\partial z}\right|_{z=h_{j}(r)-0}-\left.\frac{\partial u_{n}}{\partial z}\right|_{z=h_{j}(r)+0}\right) h_{j}^{\prime}(r) .
$$

At this point, use of the relation in Eq.(5-5) on the right-hand side of Eq.(A-6) results in

$$
\left.\frac{\partial u_{n}}{\partial r}\right|_{z=h_{j}(r)-0}-\left.\frac{\partial u_{n}}{\partial r}\right|_{z=h_{j}(r)+0}=-\left.\left(\rho_{j-1}-\rho_{j}\right)\left(\frac{1}{\rho} \frac{\partial u_{n}}{\partial z}\right)\right|_{h_{j}(r)} \frac{d h_{j}}{d r} .
$$

Multiplying both sides of Eq.(A-7) by $\Delta r$ and utilizing $\Delta u_{n} \approx \frac{\partial u_{n}}{\partial r} \Delta r$ and $\Delta h_{j} \approx \frac{d h_{j}}{d r} \Delta r$, we can finally obtain Eq.(5-15):

$$
\left.\Delta u_{n}\right|_{h_{j}-0}-\left.\Delta u_{n}\right|_{h_{j}+0}=-\left(\rho_{j-1}-\rho_{j}\right)\left(\frac{1}{\rho} \frac{\partial u_{n}}{\partial z}\right)_{h_{j}} \Delta h_{j} .
$$




\section{Appendix B}

In this appendix we will apply the simulated annealing method to the Nantucket Sound experimental data in order to estimate the local eigenvalues, which will then be compared with the local eigenvalues estimated by using the asymptotic Hankel transform with a short sliding window as well as mode filtering in Chapter 6.1.

As introduced in Chapter 1, the simulated annealing algorithm is one of the iteration of forward models methods for estimating the unknown parameters of interest. In this algorithm, we iteratively change the values of these parameters until the energy function $E$ takes a value close to its global minimum; the energy function measures the difference between the observed and simulated data and may take different forms depending on the type of data [39]. At each iterative step, we calculate the change in the energy $\Delta E$ in accordance with the changes in the values of the parameters and accept these values always if $\Delta E<0$; if, however, $\Delta E \geq 0$, we may then accept them with the probability $\Xi=\exp (-\Delta E / T)$. Owing to this process, we can avoid being trapped in the local minimums of $E$. Here $T$ is called the temperature and plays the role of a parameter controlling the probability function. Namely, we decrease $T$ gradually at each iteration of the calculation so as to reach the global minimum of $E$.

Since the energy function $E$ has to be calculated many times in this algorithm, we need an efficient means for computing the simulated field. Here we can set the local eigenvalues at selected ranges $\kappa_{n}\left(r_{j}\right)(1 \leq j \leq J)$ as unknown parameters to be estimated. Then, after interpolating the local eigenvalues between these selected ranges with the use of $\kappa_{n}\left(r_{j}\right)$, we may simulate the pressure field $p_{s}(r)$ based on adiabatic mode theory (Eq.(2-12)). If we also treat the local eigenfunctions in the amplitude as unknown parameters in the manner described above, then they can be estimated as well by using 
the annealing method; here we treat them to be range-independent in order to save computation time.

By using the measured field $p(r)$ and the simulated field $p_{s}(r)$, we here define the energy function as

$$
E=\int_{0}^{R}\left|\hat{p}(r)-\hat{p}_{s}(r)\right|^{2} d r
$$

where $\hat{p}(r)$ and $\hat{p}_{s}(r)$ are normalized pressures as

$$
\begin{aligned}
& \hat{p}(r) \equiv \frac{p(r)}{\sqrt{\int_{0}^{R}\left|p\left(r^{\prime}\right)\right|^{2} d r^{\prime}}} \\
& \hat{p}_{s}(r) \equiv \frac{p_{s}(r)}{\sqrt{\int_{0}^{R}\left|p_{s}\left(r^{\prime}\right)\right|^{2} d r^{\prime}}} .
\end{aligned}
$$

Now let us apply the simulated annealing method to the Nantucket Sound experimental data and estimate the local eigenvalues. We use the pressure data which was measured by the midcolumn receiver $\left(z_{r}=7.1\right)$. By referring to the result in Figure 6-3, a set of ranges $r_{j}(j=1, \ldots, J)$ and initial values of $\kappa_{n}\left(r_{j}\right)$ are selected as shown in Table B-1(a). In order to keep the parameter values within the interval shown in Table B-1(b), we may use the effective method described in Ref. 40. As for a cooling schedule, it is generally desirable to start with a high temperature and cool very slowly, but this requires a large amount of computation time. If we, however, use too low an initial temperature or a fast cooling rate, then we run the risk of being trapped in one of the local minima. In the process of decreasing the temperature, in general, the energy function falls off suddenly at some temperature $\left(T_{c}\right)$. Therefore if $T_{c}$ is known, we may start with the temperature slightly higher than $T_{c}$ with a very slow rate. Thus, we first find the 
temperature $T_{c}$ with a relatively fast cooling rate; and then we reset the initial temperature and iterate the process with a relatively slow cooling rate.

(1) Figure B-1 shows the result for the energy function $E$ in terms of the temperature $T$ when decreasing the temperature as $T=0.1 \times(0.99)^{l}$ ( $l$ :iteration number, see Figure B-2). From Figure B-1, we can observe that $T_{c}$ exists between 0.02 and 0.03 .

(2) Based on the result obtained in (1), we set the initial temperature to be 0.03 and the cooling schedule as $T=0.03-0.00001 \times l(l$ : iteration number $)$.

As a result of the cooling schedule in item (2), we obtain Figures B-3 through B-5. In Figure B-3, we again observe that $T_{c}$ exists between $T=0.03(l=0)$ and $0.02(l=1000)$. This figure shows that the energy function does not get close to zero, which is due to the approximation in representing the local eigenvalues with the use of segmented straight lines and also due to the fixed modal and attenuation (Table B-1(c)). Figure B-4 shows the convergence of the parameters $\kappa_{n}\left(r_{j}\right)(j=1, \ldots, J)$ with iteration number. Since the SNR for the second mode is lower than the SNR for the first mode as seen from Figure 6$1, \kappa_{2}\left(r_{j}\right)$ shows slower convergence than $\kappa_{1}\left(r_{j}\right)$. Also, as seen from the comparison of parameters with lower $j$ and higher $j$, the latter has slower convergence. This is due to the fact that the variation in the parameters with higher $j$ changes the simulated pressure field only for ranges close to the maximum range. For example, the variation of $\kappa_{n}\left(r_{J}\right)$ affects the pressure field only in the range $r_{J-1} \leq r \leq r_{J}$, whereas the variation of $\kappa_{n}\left(r_{1}\right)$ affects the pressure field in the entire range. Finally, in Figure B-5, the local eigenvalues estimated by using the simulated annealing method are compared to those obtained by using the Hankel transform with a short sliding window. This figure reveals that both results agree with each other fairly well. The disagreement in the second mode for ranges greater than $1000 \mathrm{~m}$ is due to slower convergence described above. 
Table B-1 (a) The selected ranges and initial values for the parameters $\kappa_{n}\left(r_{j}\right)$. (b) The upper and lower limits of $\kappa_{n}\left(r_{j}\right)$. (c) The relative amplitudes and mode attenuations used for the simulation.

(a)

\begin{tabular}{lrll}
$j$ & \multicolumn{1}{c}{$\begin{array}{c}r_{j} \\
(\mathrm{~m})\end{array}$} & $\begin{array}{c}\kappa_{1}\left(r_{j}\right) \\
\left(\mathrm{m}^{-1}\right)\end{array}$ & $\begin{array}{c}\kappa_{2}\left(r_{j}\right) \\
\left(\mathrm{m}^{-1}\right)\end{array}$ \\
1 & 0.0 & 0.56 & 0.49 \\
2 & 250.0 & 0.56 & 0.49 \\
3 & 400.0 & 0.56 & 0.49 \\
4 & 600.0 & 0.57 & 0.51 \\
5 & 900.0 & 0.57 & 0.51 \\
6 & 1050.0 & 0.57 & 0.51 \\
7 & 1320.0 & 0.57 & 0.52
\end{tabular}

(b)

Lower limit

Upper limit

$\begin{array}{cc}\kappa_{1}\left(r_{j}\right) & \kappa_{2}\left(r_{j}\right) \\ \left(\mathrm{m}^{-1}\right) & \left(\mathrm{m}^{-1}\right)\end{array}$

$\begin{array}{ll}0.55 & 0.47 \\ 0.59 & 0.55\end{array}$

(c)

Relative amplitude

Mode $1 \quad$ Mode 2

Mode attenuation $(\mathrm{dB} / \mathrm{m}) \quad 0.23 \times 10^{-3} \quad 1.5 \times 10^{-3}$ 


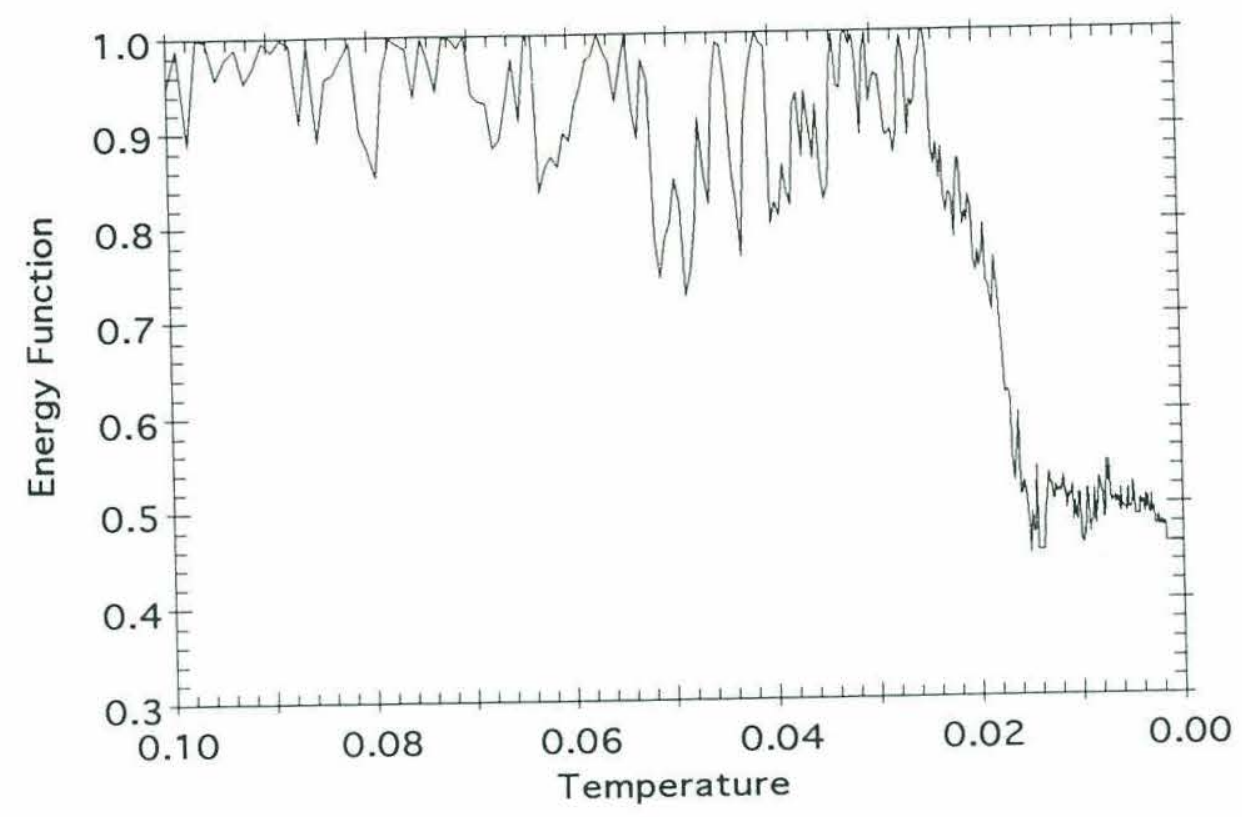

Figure B-1: Energy function versus temperature when decreasing the temperature as $T=0.1 \times(0.99)^{l}(l:$ iteration number $)$.

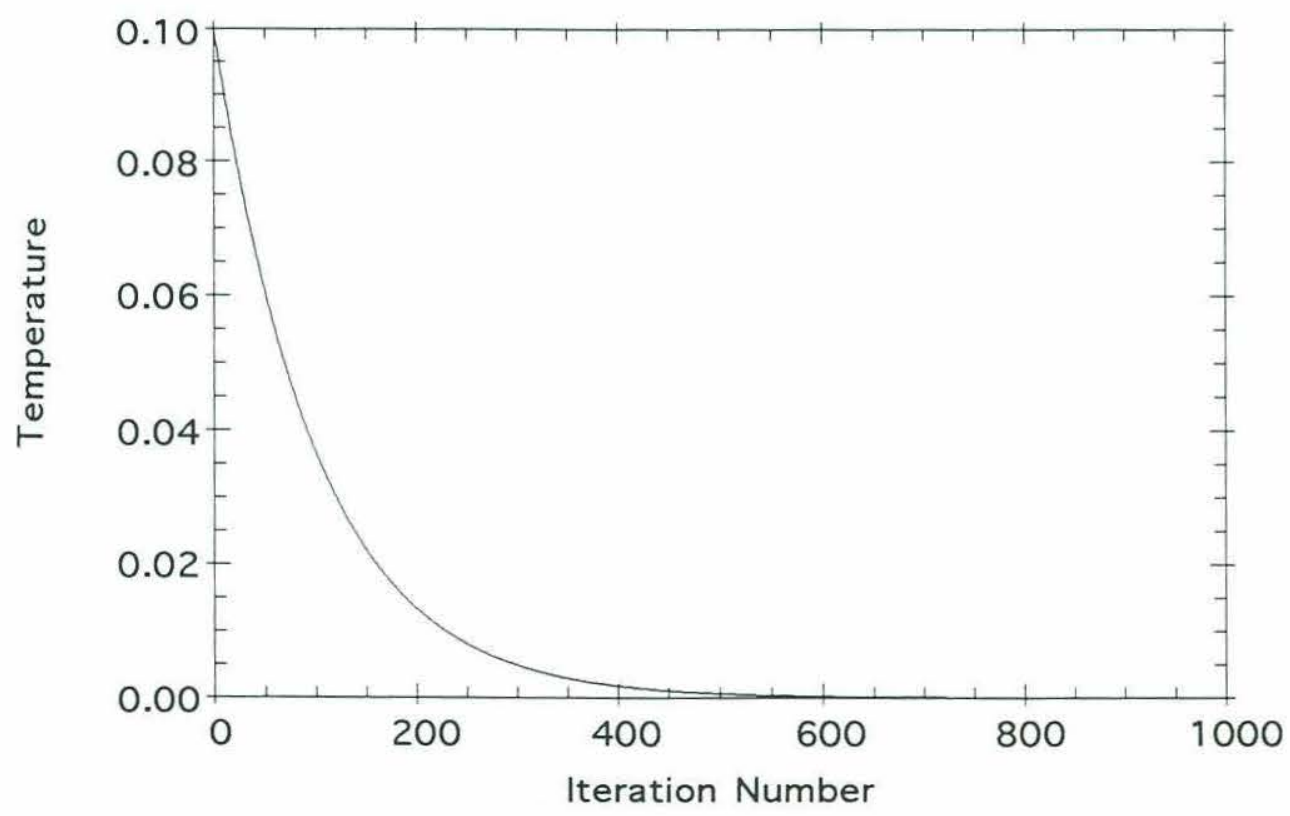

Figure B-2: Temperature in terms of iteration number when decreasing the temperature as $T=0.1 \times(0.99)^{l}$ ( $l$ : iteration number). 


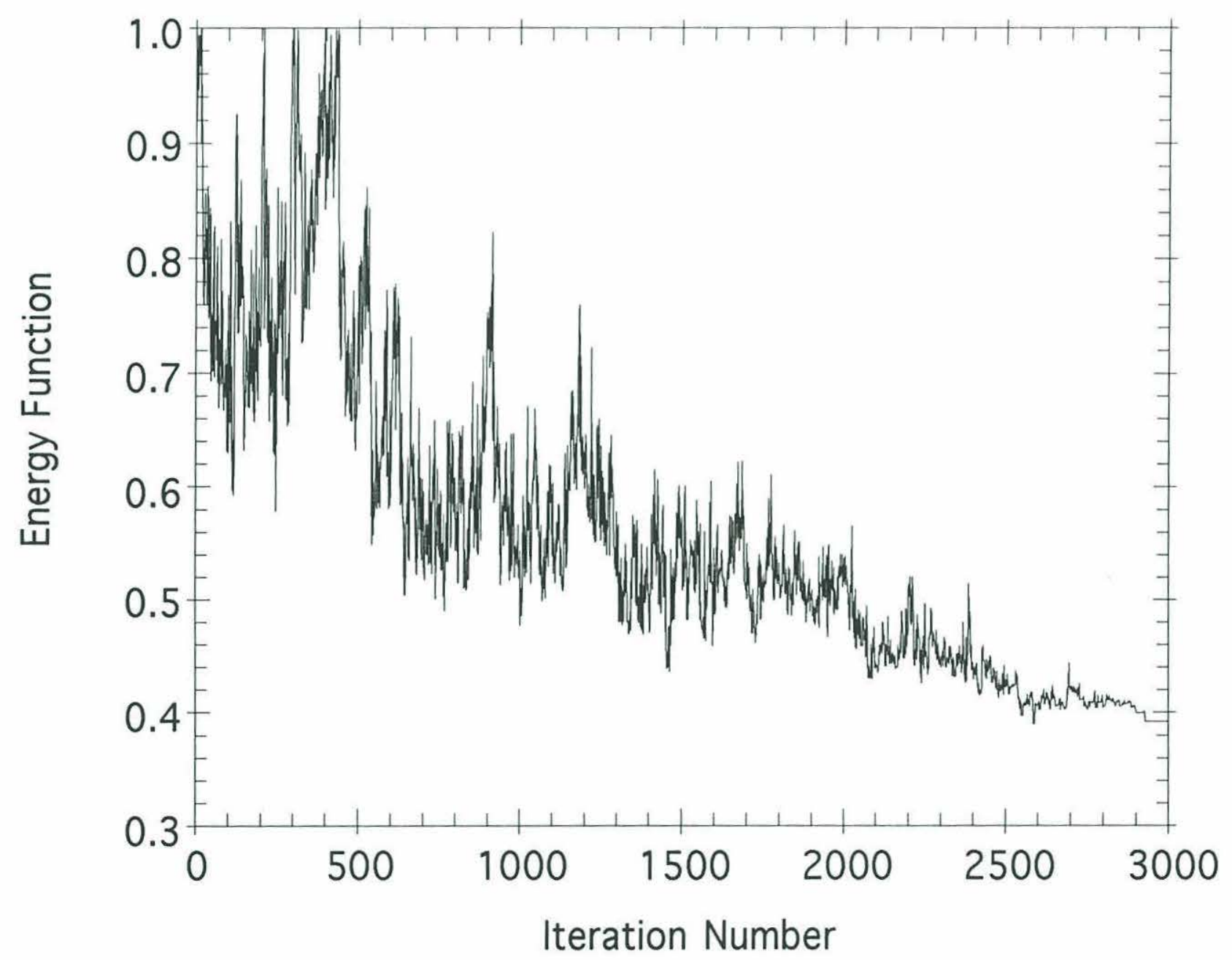

Figure B-3: Energy function in terms of iteration number when decreasing the temperature as $T=0.03-0.00001 \times l(l$ :iteration number $)$. 


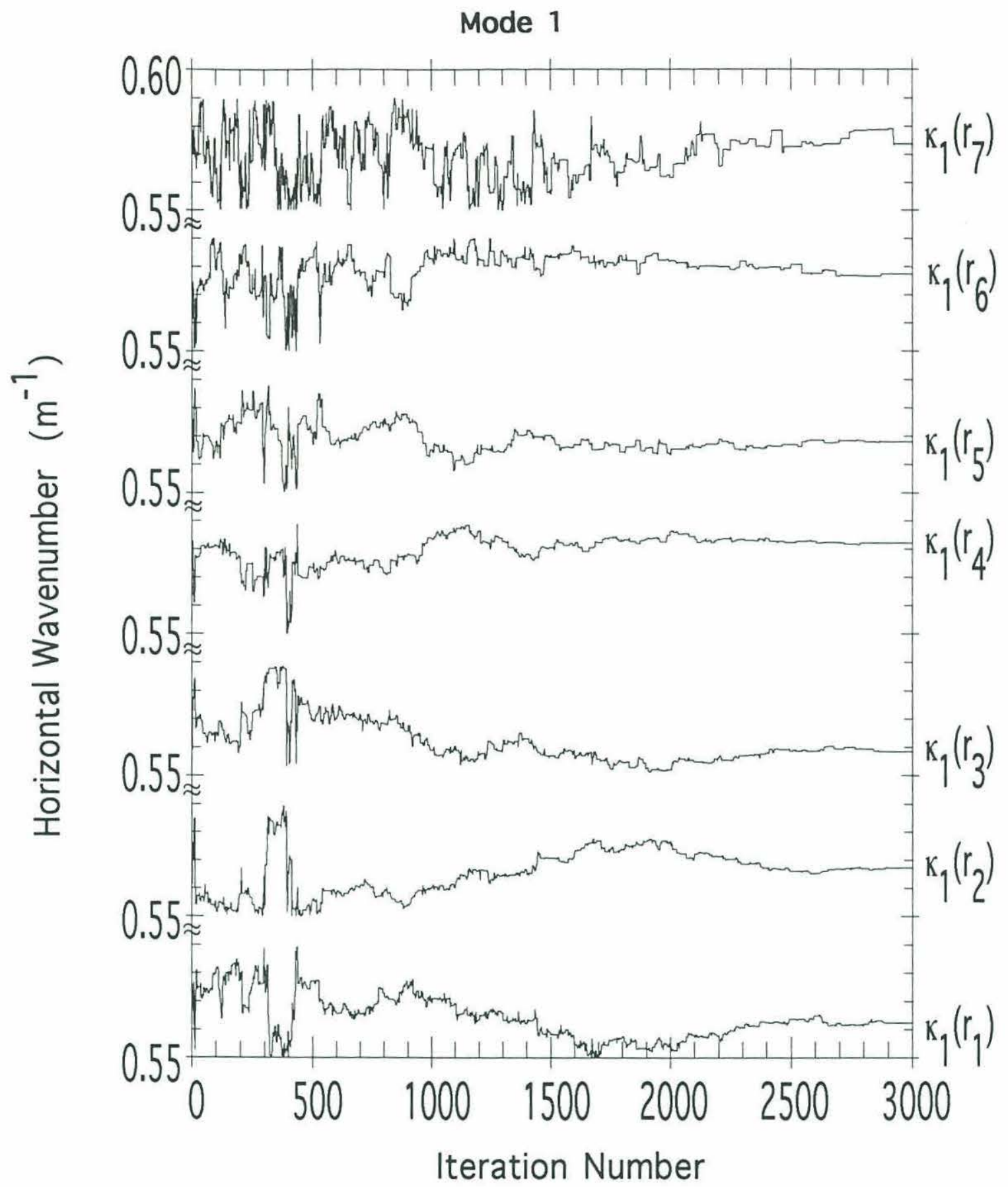

Figure B-4: Convergence of the parameters when decreasing the temperature as $T=0.03-0.00001 \times l(l$ : iteration number $)$. 


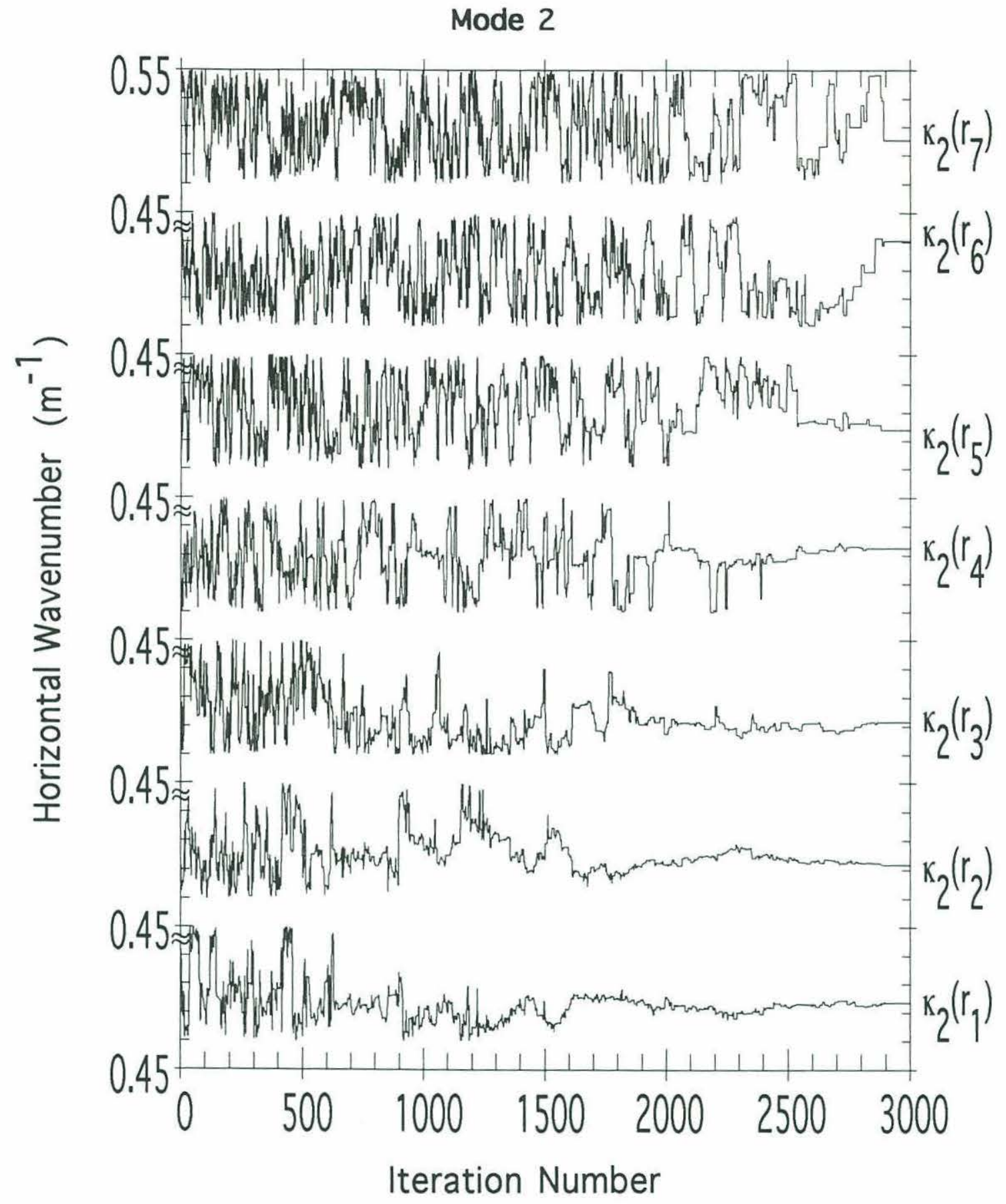

Figure B-4, continued 


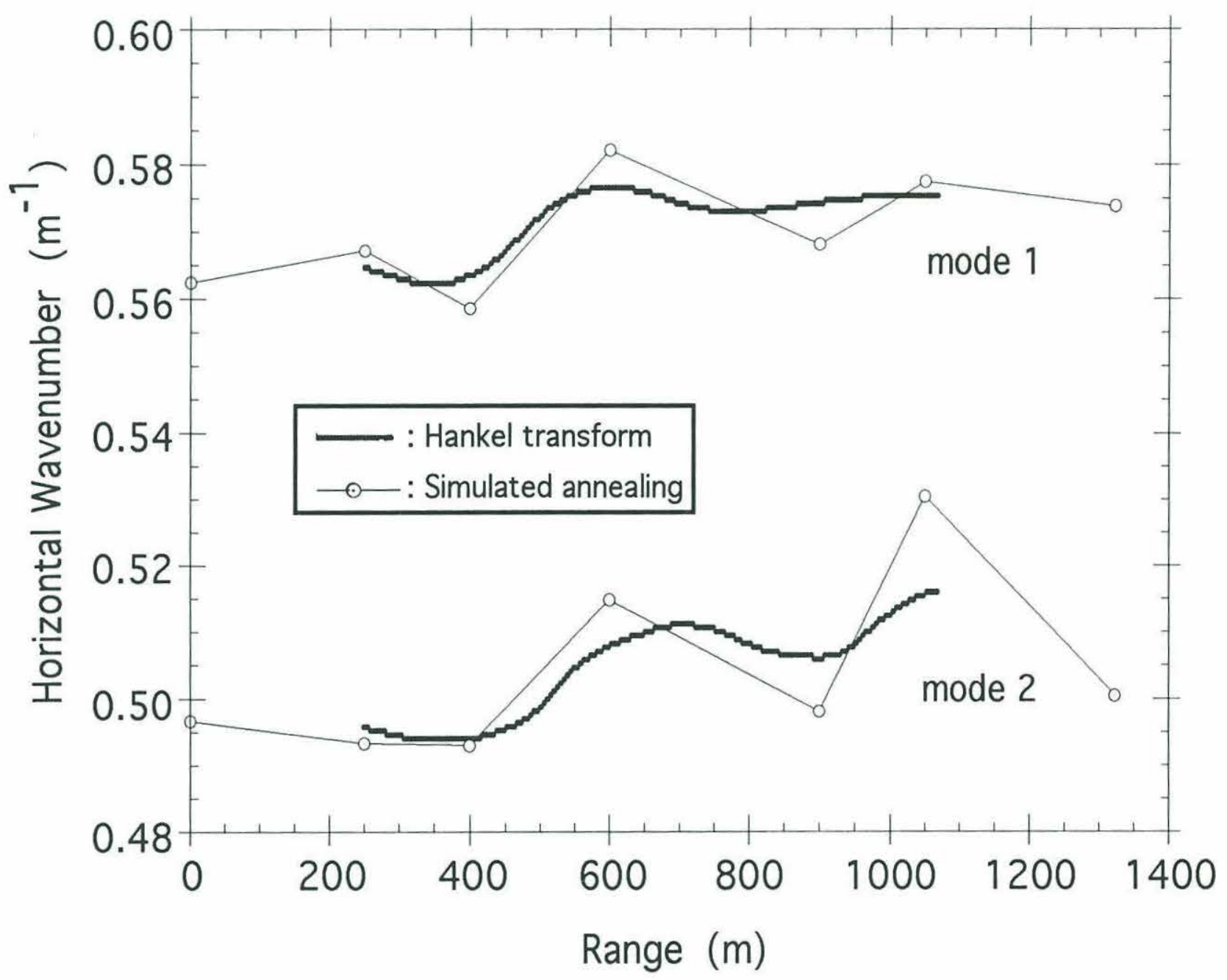

Figure B-5: Estimated local eigenvalues obtained by using the simulated annealing method with a cooling schedule $T=0.03-0.00001 \times l(l$ :iteration number). These eigenvalues are compared with those obtained in Chapter 6.1 by using the zero-order asymptotic Hankel transform with a sliding window together with mode filtering. 


\section{Appendix C}

Throughout this thesis, the ocean and seabed environment is assumed to vary gradually in the horizontal direction, so that we may utilize adiabatic mode theory. In this appendix, we will check that the range variation in the Nantucket Sound case satisfies the above assumption.

As demonstrated by Milder [59], the criterion for adiabatic mode theory is given by

$$
\frac{2\left|B_{n n}\right|}{\left|\kappa_{n}-\kappa_{m}\right|} \ll<1 \quad(m=n \pm 1)
$$

where $B_{n m}$ is the coupling coefficient defined by

$$
B_{n m}=\int_{0}^{-} \frac{1}{\rho} u_{n}(r, z) \frac{\partial}{\partial r} u_{m}(r, z) d z
$$

In order to evaluate the right-hand side in Eq.(C-2) numerically, let us use the approximation $\partial u_{m} / \partial r \approx\left[u_{m}(r+\Delta r, z)-u_{m}(r, z)\right] / \Delta r$, which yields

$$
B_{n m} \approx \frac{1}{\Delta r} \int_{0}^{H} \frac{1}{\rho} u_{n}(r, z) u_{m}(r+\Delta r, z) d z,
$$

where the orthonormality condition in Eq.(2-9) has been used and $H=40 \mathrm{~m}$.

Figure C-1 shows the values of the left hand side in Eq.(C-1) for the bottom model (Figure 6-4) inferred from the Nantucket Sound experimental data. From the result in Figure C-1, it can be seen that the adiabatic criterion, Eq.(C-1), is satisfied for this bottom model. 


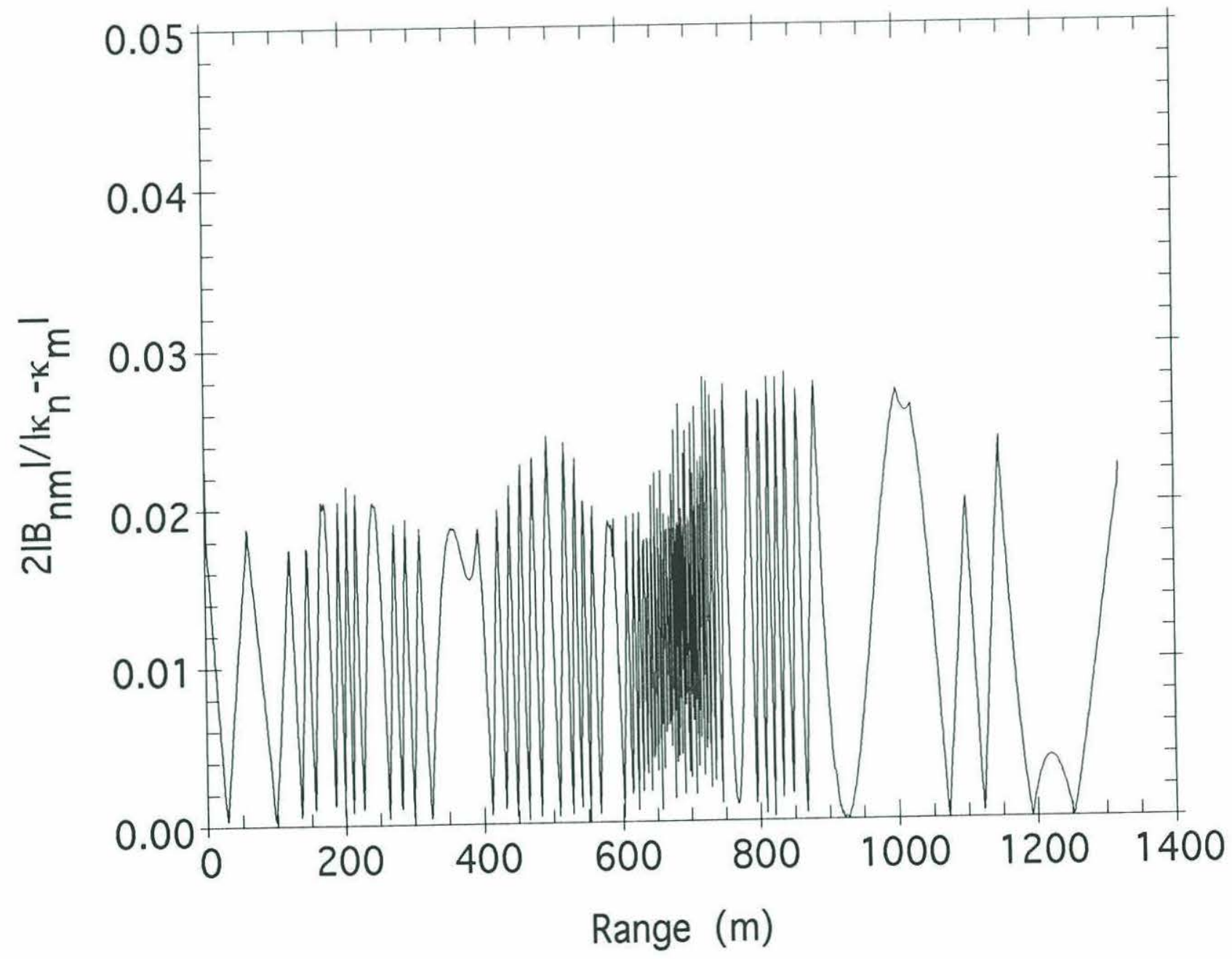

Figure C-1: Ratio of coupling coefficient $\left|B_{n m}\right|$ to the difference of eigenvalues of adjacent modes: $2\left|B_{n m}\right|\left|\kappa_{n}-\kappa_{m}\right|(n=1, m=2)$. 


\section{Bibliography}

[1] J. Zhou, X. Zhang, P. H. Rogers, and J. Jarzynski, "Geoacoustic parameters in a stratified sea bottom from shallow-water acoustic propagation," J. Acoust. Soc. Am. 82, 2068-2074 (1987)

[2] C. Feuillade, D. R. DelBalzo, and M. M. Rowe, "Environmental mismatch in shallowwater matched-field processing: geoacoustic parameter variability," J. Acoust. Soc. Am. 85, 2354-2364 (1989)

[3] F. B. Jensen and W. A. Kuperman, "Optimum frequency of propagation in shallow water environments," J. Acoust. Soc. Am. 73, 813-819 (1983)

[4] M. Porter and E. L. Reiss, "A numerical method for bottom interacting ocean acoustic normal modes," J. Acoust. Soc. Am. 77, 1760-1767 (1985)

[5] S. T. McDaniel and D. F. McCammon, "Mode coupling and the environmental sensitivity of shallow-water propagation loss predictions," J. Acoust. Soc. Am. 82, 217-223 (1987)

[6] M. J. Buckingham, "Theory of acoustic propagation around a conical seamount," J. Acoust. Soc. Am. 80, 265-277 (1986)

[7] I. T. Lu and L. B. Felsen, "Range-dependent canonical solutions for shallow ocean acoustics," in Computational Acoustics edited by D. Lee, R. L. Sternberg, and M. H. Schultz, IMACS (1988)

[8] L. A. Rubano, "Acoustic propagation in shallow water over a low-velocity bottom," J. Acoust. Soc. Am. 67, 1608-1613 (1980)

[9] C. T. Tindle, "Attenuation parameters from normal mode measurements," J. Acoust. Soc. Am. 71, 1145-1148 (1982)

[10] T. M. Brocher and J. I. Ewing, "A comparison of high-resolution seismic methods for determining seabed velocities in shallow water," J. Acoust. Soc. Am. 79, 286-298 (1986)

[11] W. A. Kuperman and F. B. Jensen, Eds., Bottom-Interacting Ocean Acoustics, Plenum, New York (1980)

[12] S. D. Rajan and G. V. Frisk, "The Effect of Seasonal Temperature Fluctuations in the Water Column on Sediment Compressional Wave Speed Profiles in Shallow Water," in Ocean Variability \& Acoustic Propagation, edited by J. P. Potter and A. Warn-Varnas, Kluwer Academic Publishers, Dordrecht (1991)

[13] L. M. Brekhovskikh, M. Leonid,Waves in Layered Media, Academic, New York (1980) 
[14] C. S. Clay and H. Medwin, Acoustical Oceanography, Wiley, New York (1977)

[15] T. Akal and J. M. Berkson, Ocean Seismo-Acoustics, Plenum, New York (1986)

[16] G. V. Frisk, Ocean and Seabed Acoustics: A Theory of Wave Propagation, PrenticeHall, Englewood Cliffs, NJ (1994)

[17] D. C. Stickler, "Negative bottom loss, critical-angle shift, and the interpretation of the bottom reflection coefficient," J. Acoust. Soc. Am. 61, 707-710 (1977)

[18] K. E. Hawker and T. L. Foreman, "A plane wave reflection loss model based on numerical integration," J. Acoust. Soc. Am. 64, 1470-1477 (1978)

[19] G. V. Frisk, "Inhomogeneous waves and the plane-wave reflection coefficient," J. Acoust. Soc. Am. 66, 219-234 (1979)

[20] G. V. Frisk, A. V. Oppenheim, and D. R. Martinez, "A Technique for measuring the plane-wave reflection coefficient of the ocean bottom," J. Acoust. Soc. Am. 68, 602612 (1980)

[21] D. R. Mook, "The Numerical Synthesis and Inversion of Acoustic Fields Using the Hankel Transform with Application to the Estimation of the Plane Wave Reflection Coefficient of the Ocean Bottom," Sc.D. Thesis, MIT/WHOI Joint Program, Cambridge, MA, and Woods Hole, MA (1983)

[22] G. V. Frisk and J. F. Lynch, "Shallow water waveguide characterization using the Hankel transform," J. Acoust. Soc. Am. 76, 205-211 (1984)

[23] M. S. Wengrovitz, "The Hilbert-Hankel Transform and Its Application to Shallow Water Ocean Acoustics," Sc.D. thesis, MIT/WHOI Joint Program, Cambridge, MA, and Woods Hole, MA (1986)

[24] C. S. Clay, "Array steering in a layered waveguide," J. Acoust. Soc. Am. 33, 865870 (1961)

[25] C. S. Clay, "Use of arrays for acoustic transmission in a noisy ocean," Rev. Geophys. 4, 475-507 (1966)

[26] R. H. Ferris, "Comparison of measured and calculated normal-mode amplitude functions for acoustic waves in shallow water," J. Acoust. Soc. Am. 52, 981-988 (1972)

[27] J. F. Lynch, "On the use of focused horizontal arrays as mode separation and source localization devices in ocean acoustics. part I: theory," J. Acoust. Soc. Am. 74, 14061417 (1983)

[28] W. A. Kuperman, M. F. Werby, K. E. Gilbert, and G. J. Tango, "Beam forming on bottom-interacting tow-ship noise," IEEE J. Oceanic Engineering OE-10, 290-298 (1985) 
[29] J. F. Lynch, S. D. Rajan, and G. V. Frisk, "A comparison of broadband and narrowband modal inversions for bottom geoacoustic properties at a site near Corpus Christi, Texas," J. Acoust. Soc. Am. 89, 648-665 (1991)

[30] G. V. Frisk, J. F. Lynch, and S. D. Rajan, "Determination of compressional wave speed profiles using modal inverse techniques in a range-dependent environment in Nantucket Sound," J. Acoust. Soc. Am. 86, 1928-1939 (1989)

[31] J. A. Mercer, "Geoacoustic parameters and depth dependent Green's function for a silty sediment at $100 \mathrm{~Hz}$ in shallow water," J. Acoust. Soc. Am. 83, 611-616 (1988)

[32] G. V. Frisk, K. Ohta, S. D. Rajan, and J. A. Doutt, "The measurement of modal evolution in a range-dependent, shallow-water waveguide," J. Acoust. Soc. Am. Suppl. 2 91, S2465 (1992)

[33] F. J. Diemer, "A Prony Algorithm for Shallow Water Waveguide Analysis," O.E. Thesis, MIT/WHOI Joint Program, Cambridge, MA, and Woods Hole, MA (1987)

[34] G. V. Frisk, "Inverse Methods in Ocean Bottom Acoustics," in Oceanographic and Geophysical Tomography, edited by Y. Desaubies, A. Tarantola, and J. Zinn-Justin, Elsevier Science Publishers, BV (1990)

[35] G. V. Frisk, J. A. Doutt, and E. E. Hays, "Geoacoustic models for the Icelandic Basin," J. Acoust. Soc. Am. 80, 591-600 (1986)

[36] G. V. Frisk, J. F. Lynch, and J. A. Doutt, "The Determination of Geoacoustic Models in Shallow Water," in Ocean Seismo-Acoustics, edited by T. Akal and J. M. Berkson, Plenum, New York (1986)

[37] S. Kirkpatrick, C. D. Gelatt, M. P. Vecchi, "Optimization by simulated annealing," Science 220, 671-680 (1983)

[38] D. H. Rothman, "Nonlinear inversion, statistical mechanics, and residual statistics estimation," Geophysics 50, 2797-2807 (1985)

[39] M. K. Sen and P. L. Stoffa, "Nonlinear one-dimensional seismic waveform inversion using simulated annealing," Geophysics 56, 1624-1638 (1991)

[40] M. D. Collins, W. A. Kuperman and H. Schmidt, "Nonlinear inversion for oceanbottom properties," J. Acoust. Soc. Am. 92, 2770-2783 (1992)

[41] R. L. Parker, "Understanding inverse theory," Ann. Rev. Earth Planet. Sci. 5, 35-64 (1977)

[42] W. Menke, Geophysical Data Analysis: Discrete Inverse Theory, Academic Press, New York (1984)

[43] K. Aki and P. G. Richards, Quantitative Seismology: Theory and Methods (Vol.2), Freeman, San Francisco (1980) 
[44] A. A. Merab, "Exact Reconstruction of Ocean Bottom Velocity Profiles from Monochromatic Scattering Data," Sc.D. Thesis, MIT/WHOI Joint Program, Cambridge, MA, and Woods Hole, MA (1987)

[45] D. C. Stickler, "Inverse scattering in a stratified medium," J. Acoust. Soc. Am. 74, 994-1005 (1983)

[46] I. M. Gelfand and B. M. Levitan, "On the determination of a differential equation from its spectral function," Izv. Akad. Nauk SSSR 15, 309-360 (1951); Am. Math. Soc. Transl. 1, 253-304 (1955)

[47] L. I. Schiff, Quantum Mechanics, McGraw-Hill, New York (1968)

[48] S. D. Rajan, "An Inverse Method for Obtaining the Attenuation Profile and Small Variations in the Sound Speed and Density Profiles of the Ocean Bottom," Ph.D. thesis, MIT/WHOI Joint Program, Cambridge, MA, and Woods Hole, MA (1985)

[49] S. D. Rajan and G. V. Frisk, "An Inverse Method for Obtaining the Attenuation Profile and Small Variations in the Sound Speed and Density Profiles of the Ocean Bottom," in Progress in Underwater Acoustics, edited by H.M. Merklinger, Plenum, New York (1987)

[50] S. D. Rajan, J. F. Lynch, and G. V. Frisk, "Perturbative inversion methods for obtaining bottom geoacoustic parameters in shallow water," J. Acoust. Soc. Am. 82, 998-1017 (1987)

[51] J. F. Lynch, S. D. Rajan, and G. V. Frisk, "A Perturbative Inverse Method for the Determination of Geoacoustic Parameters in Shallow Water," in Progress in Underwater Acoustics, edited by H. M. Merklinger, Plenum, New York (1987)

[52] C. B. Officer, Introduction to the Theory of Sound Transmission, McGraw-Hill, New York (1958)

[53] H. P. Bucker, "Sound propagation in a channel with lossy boundaries," J. Acoust. Soc. Am. 48, 1187-1194 (1970)

[54] H. Otsubo, K. Ohta, and S. Ozaki, "Normal-mode solution in the ocean with absorbing bottom sediments which have a sound-speed gradient," J. Acoust. Soc. Jpn. (E), 1, 47-57 (1980)

[55] D. C. Stickler, "Normal-mode program with both the discrete and branch line contributions," J. Acoust. Soc. Am. 57, 856-861 (1975)

[56] M. K. Macpherson and G. V. Frisk, "The contribution of normal modes in the bottom to the acoustic field in the ocean," J. Acoust. Soc. Am. 68, 929-940 (1980) [57] M. Abramowitz and J. A. Stegun, Handbook of Mathematical Functions, National Bureau of Standards, Washington, D.C. (1965) 
[58] D. E. Weston, "Guided propagation in a slowly varying medium," Proc. Phys. Soc. (London) 73, 365-384 (1959)

[59] D. M. Milder, "Ray and wave invariants for SOFAR channel propagation," J. Acoust. Soc. Am. 46, 1259-1263 (1969)

[60] S. R. Rutherford and K. E. Hawker, "An examination of the influence of the range dependence of the ocean bottom on the adiabatic approximation," J. Acoust. Soc. Am. 66, 1482-1486 (1979)

[61] A. D. Pierce, "Extension of the method of normal modes to sound propagation in an almost-stratified medium," J. Acoust. Soc. Am. 37, 19-27 (1965)

[62] L. Brekhovskikh and Yu. Lysanov, Fundamentals of Ocean Acoustics, SpringerVerlag, Berlin (1982)

[63] A. Nagl, H. Überall, A. J. Hang, and G. L. Zasure, "Adiabatic mode theory of underwater sound propagation in a range-dependent environment," J. Acoust. Soc. Am. 63, 739-749 (1978)

[64] J. S. Perkins and R. N. Baer, "An approximation to the three-dimensional parabolicequation method for acoustic propagation," J. Acoust. Soc. Am. 72, 515-522 (1982)

[65] W. A. Kuperman, M. B. Porter, J. S. Perkins, and R. B. Evans, "Rapid computation of acoustic fields in three-dimensional ocean environments," J. Acoust. Soc. Am. 89, 125-133 (1991)

[66] H. Weinberg and R. Burridge, "Horizontal ray theory for ocean acoustics," J. Acoust. Soc. Am. 55, 63-79 (1974)

[67] L. Brekhovskikh and O. A. Godin, Acoustics of Layered Media II, Springer-Verlag, Berlin (1992)

[68] F. R. DiNapoli and R. L. Deavenport, "Theoretical and Numerical Green's Function Field Solution in a Plane Multilayered System," J. Acoust. Soc. Am. 67, 92-105 (1980)

[69] H. Schmidt and F. B. Jensen, "A full wave solution for propagation in multilayered viscoelastic media with application to Gaussian beam reflection at fluid-solid interfaces," J. Acoust. Soc. Am. 77, 813-825 (1985)

[70] G. F. Carrier, M. Krook, and C. E. Pearson, Functions of a Complex Variable, McGraw-Hill, New York (1966)

[71] B. Boashasb, "Time-Frequency Signal Analysis," in Advances in Spectrum Analysis and Array Processing, edited by S. Haykin, Prentice Hall, NJ (1991)

[72] L. Cohen, "Time-frequency distributions - a review," IEEE Proceedings, 77, 941981 (1989) 
[73] N. Bleistein and R. A. Handelsman, Asymptotic Expansions of Integrals, Dover Publications, New York (1975)

[74] C. T. Tindle, K. M. Guthrie, G. E. J. Bold, M. D. Johns, D. Jones, K. O. Dixon, and T. G. Birdsall, "Measurements of the frequency dependence of normal modes," J. Acoust. Soc. Am. 64, 1178-1187 (1978)

[75] E. Cen, J. Zhou, and E. Shang, "Normal mode filtering in shallow water," J. Acoust. Soc. Am. 74, 1833-1836 (1983)

[76] T. C. Yang, "A method of range and depth estimation by modal decomposition," J. Acoust. Soc. Am. 82, 1736-1745 (1983)

[77] R. A. Wiggins, "The general linear inverse problem: implication of surface waves and free oscillations for earth structure," Rev. Geophys. Space Phys. 10, 251-285 (1972)

[78] F. B. Hildebrand, Advanced Calculus for Applications, Prentice-Hall, Englewood Cliffs, NJ (1976)

[79] K. Ohta, "Horizontal refraction of normal modes due to variation in bathymetry and subbottom sediment structure," J. Acoust. Soc. Am. Suppl.1 84, S151 (1988)

[80] A. V. Oppenheim and R. W. Schafer, Digital Signal Processing, Prentice-Hall, NJ (1975)

[81] J. D. Milliman, Z. Jiezao, L. Anchun, J. I. Ewing, "Late quaternary sedimentation on the outer and middle New Jersey continental shelf: result of two local deglaciations?" Jour. Geology, 98, 966-976 (1990)

[82] D. J. P. Swift, R. Moir, and G. L. Freeman, "Quaternary rivers on the New Jersey shelf: relation of seafloor to buried valleys," Geology 8, 276-280 (1980)

[83] S. D. Rajan, G. V. Frisk, and J. F. Lynch, "On the determination of modal attenuation coefficients and compressional wave attenuation profiles in a rangedependent environment in Nantucket Sound," IEEE J. Oceanic Engineering OE-17, 118-128 (1992)

[84] E. L. Hamilton, "Geoacoustic modeling of the sea floor," J. Acoust. Soc. Am. 68, $1313-1340$ (1980)

[85] W. Carey, L. M. Dillman, and J. Doutt, "Shallow -Water Transmission

Measurements Taken on the New Jersey Continental Shelf," NUWC-NL Technical Document 10,025, New London, CT (1992)

[86] L. M. Dillman, "An Experimental Investigation of an Acoustic Technique to Determine Shallow Water Bottom Boundary Impedance," NUWC-NL Technical Document 10,045, New London, CT (1992) 